\title{
Portal dosimetry in radiotherapy
}

Citation for published version (APA):

Nijsten, S. M. J. J. G. (2009). Portal dosimetry in radiotherapy. [Doctoral Thesis, Maastricht University]. Datawyse / Universitaire Pers Maastricht. https://doi.org/10.26481/dis.20090520sn

Document status and date:

Published: 01/01/2009

DOI:

10.26481/dis.20090520sn

Document Version:

Publisher's PDF, also known as Version of record

\section{Please check the document version of this publication:}

- A submitted manuscript is the version of the article upon submission and before peer-review. There can be important differences between the submitted version and the official published version of record.

People interested in the research are advised to contact the author for the final version of the publication, or visit the DOI to the publisher's website.

- The final author version and the galley proof are versions of the publication after peer review.

- The final published version features the final layout of the paper including the volume, issue and page numbers.

Link to publication

\footnotetext{
General rights rights.

- You may freely distribute the URL identifying the publication in the public portal. please follow below link for the End User Agreement:

www.umlib.nl/taverne-license

Take down policy

If you believe that this document breaches copyright please contact us at:

repository@maastrichtuniversity.nl

providing details and we will investigate your claim.
}

Copyright and moral rights for the publications made accessible in the public portal are retained by the authors and/or other copyright owners and it is a condition of accessing publications that users recognise and abide by the legal requirements associated with these

- Users may download and print one copy of any publication from the public portal for the purpose of private study or research.

- You may not further distribute the material or use it for any profit-making activity or commercial gain

If the publication is distributed under the terms of Article $25 \mathrm{fa}$ of the Dutch Copyright Act, indicated by the "Taverne" license above, 


\section{PORTAL DOSIMETRY IN RADIOTHERAPY}

Sebastiaan M.J.J.G. Nijsten 


\section{Cover illustration}

Twodimensional transit gamma images showing the agreement between predicted and measured portal dose images in the EPID plane. Blue and red colors mark the regions where gamma values are not satisfying a local dose-difference criterion of $5 \%$ or a distance-to-agreement criterion of $5 \mathrm{~mm}$. These images are obtained by applying the methods from Chapters $4-5$ in clinical practice to all patients treated with a curative intent by MAASTRO CLINIC.

\section{Production}

Cover: $\quad$ S.M.J.J.G. Nijsten

Printing: Datawyse | Universitaire Pers Maastricht

ISBN: $\quad 978-90-5278-818-0$

\section{Copyrights}

(C) Copyright S.M.J.J.G. Nijsten, Maastricht 2009

Chapter 2: $\quad$ Medical Physics, American Institute of Physics, 2004

Chapter 3: $\quad$ Radiotherapy and Oncology, Elsevier Ireland Ltd, 2007

Chapter 4: $\quad$ Medical Physics, American Institute of Physics, 2005

Chapter 5: $\quad$ Medical Physics, American Institute of Physics, 2007

Chapter 6: $\quad$ Medical Physics, American Institute of Physics, 2006

Chapter 7: $\quad$ Medical Physics, American Institute of Physics, 2009

No parts of this publication may be reproduced in any form without prior written permission of the holder of the copyrights. 


\section{PORTAL DOSIMETRY IN RADIOTHERAPY}

\section{PROEFSCHRIFT}

ter verkrijging van de graad van doctor aan de Universiteit Maastricht, op gezag van de Rector Magnificus

Prof. mr. G.P.M.F. Mols,

volgens het besluit van het College van Decanen,

in het openbaar te verdedigen,

op woensdag 20 mei 2009 om 14.00 uur

door

\section{Sebastiaan M.J.J.G. Nijsten}

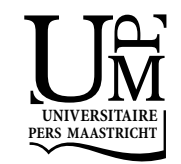




\section{Promotor}

Prof. dr. Ph. Lambin

\section{Copromotores}

Dr. ir. A.L.A.J. Dekker

Dr. B.J. Mijnheer

Dr. ir. A.W.H. Minken (RISO Deventer)

\section{Beoordelingscommissie}

Prof. dr. J. Wildberger (voorzitter)

Prof. dr. ir. B.M. ter Haar Romeny (TU/e Eindhoven, UM)

Prof. dr. M.B. van Herk (NKI/AvL Amsterdam)

Prof. J. Pouliot (UCSF San Francisco)

Dr. F. Verhaegen

Financial support by Siemens Nederland NV for the publication of this thesis is gratefully acknowledged. 


\section{CONTENTS}

\section{Introduction}

Chapter 1 Introduction and outline of the thesis

\section{Point dose verification methods and application}

Chapter 2 Verification of treatment parameter transfer by means of electronic portal dosimetry

Chapter 3 Routine individualized patient dosimetry using electronic portal imaging devices

\section{Two-dimensional transit dosimetry methods and application}

Chapter 4 Experimental verification of a portal dose prediction model

Chapter 5 A global calibration model for $a$-Si EPIDs used for transit dosimetry

Chapter 6 Comparison of ghosting effects for three commercial $a$-Si EPIDs

Chapter 7 Prediction of DVH parameter changes due to set-up errors for breast cancer treatment based on 2D portal dosimetry

\section{Discussion}

Chapter 8 General discussion and future perspectives

Appendix MAASTRO implementation of large-scale 2D portal dosimetry

List of abbreviations

Summary/samenvatting

Dankwoord

Curriculum Vitae 



\title{
INTRODUCTION
}

\author{
POINT DOSE VERIFICATION \\ METHODS AND APPLICATION
}

TWO-DIMENSIONAL TRANSIT DOSIMETRY METHODS AND APPLICATION

DISCUSSION 

CHAPTER

Introduction and outline of the thesis 



\subsection{CANCER AND RADIOTHERAPY}

Cancer is one of the major public health problems in Europe, the United States and other countries in the western world. In 2006, there were over two million incident cases of cancer in the European Union and over one million cancer deaths ${ }^{1}$. In the United States, one out of four deaths is due to cancer as estimated for $2007^{2}$. Prostate cancer (20\%-29\%) and lung cancer (15\%-17\%) are the most common forms of cancer in men while for women, breast cancer $(26 \%-29 \%)$ is diagnosed most frequently ${ }^{1,2}$. Lung cancer causes most cancer deaths in men with an estimate of $26.3 \%$ of all cancer deaths in Europe in 2006. In that year, breast cancer was the most common cause of cancer death in women $(16.7 \%)^{1}$.

Radiotherapy is one of the main treatment methods for cancer next to surgery, chemotherapy and hormone therapy. Radiotherapy (alone or in combination with other treatments) is a curative treatment for $40 \%$ of the cancer patients receiving $i^{3}$. The goal of radiotherapy is to irradiate tumor tissue to a high dose while sparing the surrounding normal healthy tissue as much as possible to limit the complications of a patient treatment. In general, irradiation is done by using external beam radiotherapy or brachytherapy.

In case of external beam radiotherapy, a radiation beam is pointed at a particular part of the body. By using multiple beams in an optimum beam angle configuration, the dose in healthy tissue can be diminished. Different types of radiation have different interactions with tissue and will cause more or less biological damage. Most commonly used radiation types are megavoltage X-rays (Megavolt or MV photon beams), electrons and protons.

In case of brachytherapy, a radioactive source is placed inside or next to the area requiring treatment. Examples of brachytherapy are interstitial brachytherapy with iodine-125 seeds implanted in the prostate to treat localized prostate cancer disease and intracavitary brachytherapy with iridium-192 placed in the cervix to irradiate cervical tumor tissue.

\subsection{EXTERNAL MEGAVOLT PHOTON BEAM TREATMENT}

Worldwide, the most frequently applied radiotherapy technique is an external MV photon beam treatment with linear accelerators (LINACs) ${ }^{4-6}$. With these devices, electrons are generated and accelerated to high energies of 4-25 MeV. The accelerated electrons produce X-rays when they collide with a tungsten target and the resulting photon beam can be used for treatment after additional filtering, collimation and shielding of the beam in the treatment head. Beam shielding is a prerequisite in high dose-high precision radiotherapy in order to obtain dose distributions that conform to the tumor volume while sparing neighbouring healthy tissue. Both individual moulded blocks and a multileaf collimator (MLC) can be used for beam shielding. The latter device is located inside the treatment head of a LINAC and consists typically of a series 


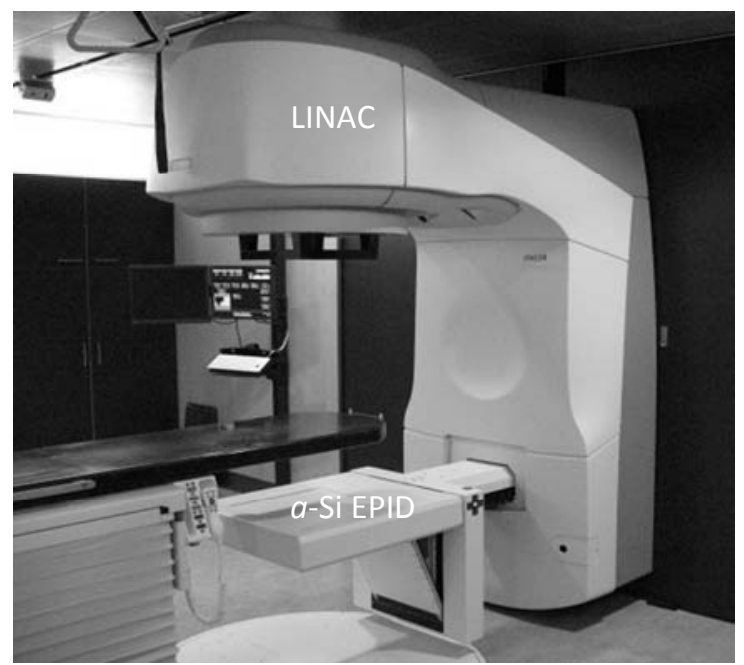

Figure 1: A Siemens Oncor medical linear accelerator (Siemens Medical Solutions, Concord, CA) equipped with an amorphous silicon ( $a$-Si) electronic portal imaging device.

of 80 to 160 metallic leaves which can be positioned individually to shape the beam aperture. Furthermore, modern LINACs are usually equipped with an electronic portal imaging device (EPID) which allows imaging of the high energetic MV photon beam that exits the patient during treatment ${ }^{7,8}$. These images can be used for patient set-up verification or detection of organ motion but also for dosimetric verification of a treatment which is called portal dosimetry. In Fig. 1, a Siemens Oncor medical linear accelerator (Siemens Medical Solutions, Concord, CA) is shown equipped with an amorphous silicon ( $a$-Si) electronic portal imaging device.

Before the treatment can be applied to a patient, an individual treatment plan is generated using a treatment planning system $(T P S)^{9}$. This system uses three-dimensional (3D) imaging information of a patient to model the position and shape of both tumor and healthy tissues. Based on this localization, an optimum beam configuration including photon beam energies, field sizes, shielding, beam directions and relative beam weighting can be determined. The end result is a definition of beam parameters that is needed to set-up the linear accelerator and the 3D dose distribution inside the patient that documents the prescribed radiotherapy treatment.

\subsection{TREATMENT VERIFICATION USING DOSE MEASUREMENTS}

By administering radiotherapy in a fractionated manner (i.e. giving every day a small dose of radiation), a small difference in biological radiation sensitivity between tumor and healthy tissue can be exploited to increase the overall tumor dose. Hence, tumor control probability can be increased with acceptable normal tissue complication 
probability. Consequently, every fraction should be given in a reproducible way and the dose delivery in a patient should be as close as possible to the prescribed dose as calculated with the TPS. For instance, Mijnheer et al. ${ }^{10}$ proposed an accuracy requirement in absorbed dose delivery at the dose specification point of $3.5 \%$ (1 SD) for external beam treatments based on the steepness of dose-effect curves for local tumor control and normal tissue damage. In order to achieve such a high accuracy, it is of utmost importance that the dose delivery is verified during external MV photon beam treatment.

In case of image-guided radiation therapy (IGRT), imaging devices are used to determine the position of tumor and healthy tissues just prior to or during treatment and to calculate positional corrections based on measured images. By applying for instance a correction to the patient position, differences in patient set-up, and hence inaccuracies in delivered dose, should consequently decrease. Examples of IGRT devices in the treatment room are EPIDs, megavoltage or kilovoltage cone-beam computed tomography (CT) scanners and CT on rails ${ }^{7,8,11-14}$.

In case of dose-guided radiation therapy (DGRT), a treatment is adapted based on direct dose measurements in combination with the results of IGRT procedures. Dose measurements can be done prior to treatment (pre-treatment measurements) and during treatment (in vivo measurements) using several dose verification devices. Diodes, thermoluminescent dosimeters (TLDs) and metal oxide semiconductor field-effect transistors (MOSFETs) ${ }^{15-18}$ are point detectors, only allowing a single point measurement. However, these detectors have a long history of clinical use, their dosimetric characteristics are well known and they are adequate for verification of conventional treatment techniques ${ }^{19-21}$. Radiographic and radiochromic film ${ }^{22}$ can be used to verify dose distributions in two dimensions and have a high spatial resolution. Therefore, film has especially been used for the verification of intensity-modulated radiation therapy (IMRT) treatments where high dose gradients are present in the plane of the beam ${ }^{23}$. The disadvantage of film is that the dose evaluation is time consuming and is prone to errors during processing, digitizing and analyzing. Gel dosimetry $^{24,25}$ is one of the few dosimetry systems currently available allowing a measurement of the 3D dose distribution. However, the method is limited by the complex preparation process and the expensive analysis using a magnetic resonance imaging (MRI) scanner.

Finally, different types of EPIDs ${ }^{7,8}$ have been developed in the past and their use for dosimetry has been investigated ${ }^{26}$. Compared with other dosimetry devices, EPIDs are already attached to a linear accelerator and no additional hardware is needed to perform portal dosimetry. EPID measurements can be performed with minimum set-up requirements and a $2 \mathrm{D}$ dose conversion can be done immediately using the digital images. Furthermore, the same EPIDs are already used for IGRT applications, e.g. for patient set-up verification using conventional portal imaging or megavoltage cone-beam CT. Consequently, one measurement device can be used to obtain different types of information during treatment. Although an EPID image contains 2D and not 3D information, it is still possible to reconstruct the 3D dose distribution inside 
a patient by using a back-projection procedure of the measured portal dose image (PDI) into three dimensions ${ }^{27-29}$.

\subsection{AIM OF THIS WORK}

The use of electronic portal imaging devices for treatment verification using dose measurements looks very promising. Until now, EPIDs have not been used clinically on a large scale for dosimetric verification and hence, clinical experience is limited. Because point detectors have a long history of clinical use, dose differences resulting from these measurements are widely reported and understood. Moving directly from these measurements to 3D dose reconstruction using EPIDs is a large step, requiring not only complex mathematical models but also a new way of comparing and visualizing dose verification results. Although a comparison between a prescribed and delivered 3D dose distribution is the ultimate goal of portal dosimetry, a more gradual clinical transition may be worthwhile ${ }^{30}$. Starting with verification procedures based on portal dose measurements in one point offers the possibility to directly compare the EPID results to the results of diode, TLD or MOSFET measurements ${ }^{19-21}$. Also, pitfalls of the new procedures can be more easily recognized and eliminated in this case. For departments already performing in vivo dosimetry measurements, it is a small step to move to an EPID based dose verification procedure that takes less time on a linear accelerator than the traditional point dose measurements.

The aim of this work was to develop dose verification methods using portal dosimetry based on point dose measurements and dose distributions in two dimensions. Both dosimetric calibration models for different types of EPIDs and prediction models to obtain reference dose values had to be implemented. All models should be based on absolute dose in water measured under full-scatter conditions, simplifying a future back-projection procedure of the measured portal dose into three dimensions. The accuracy and clinical applicability of the dose verification methods was investigated and results are presented from large-scale clinical use. The final goal was to show that routine portal dosimetry is feasible and that detection of a wider variety of dose delivery errors than the traditional point dosimeters can be accomplished.

\subsection{OUTLINE OF THE THESIS}

Chapter 1 is a general introduction to the thesis, and describes the aim of this work and the outline of the thesis. Chapters 2-3 describe the methods and application of point dose verification and chapters 4-7 describe two-dimensional transit dosimetry.

\section{Point dose verification methods and application}

Chapter 2 describes a pre-treatment dose verification procedure that verifies the portal dose at the center of open and wedged fields. Besides verification of dose, the 
same EPID was used to verify geometrical beam properties, thereby allowing the use of only one EPID image for verification of treatment parameter transfer. This is especially important when data transfer between TPS and linear accelerator is not carried out electronically.

Chapter 3 shows the clinical results of routine pre-treatment verification and individual patient dosimetry. All procedures are based on portal dosimetry and carried out by radiation therapy technicians who acquired and analysed over 37500 images from 2511 patients and 3146 treatment plans. Potential sources of dose delivery errors in one point are obtained using one dose verification solution only and are discussed in detail.

In both chapters, a dosimetric calibration model is applied to convert grayscale EPID images obtained with different video-based EPIDs into 2D absolute dose distributions. Reference central field dose values in the EPID plane were predicted by using dose prediction models independent of the TPS. For a point dose comparison at a radiological depth of $5 \mathrm{~cm}$ inside a patient, the predicted dose from the treatment planning system was compared to the measured in vivo dose obtained by back-projecting the measured transit dose.

\section{Two-dimensional transit dosimetry methods and application}

Chapter 4 describes the extension of the point dose prediction model for transit dose discussed in chapter 3 to a 2D portal dose prediction model. The 2D model is based solely on the radiological thickness of the patient along a ray line and a pre-treatment portal dose image. To use this model in the future for 3D dose reconstruction, the model has to be able to extract the primary portal dose from a measured PDI during treatment. The accuracy of the 2D portal dose prediction and the extraction of primary portal dose were therefore tested elaborately in an experimental study.

Chapter 5 describes again a dosimetric calibration model to obtain measured 2D portal dose distributions but now for indirect-detection amorphous silicon ( $a$-Si) type of EPIDs, which are nowadays most popular worldwide. A global calibration model was developed taking into account all relevant EPID response corrections. The model includes an energy spectrum correction model and had to be accurate out-of-field, because the dose in healthy tissue and the integral patient dose are increasingly important due to the application of IMRT treatments.

Chapter 6 can be seen as an addition to chapter 5 and compares ghosting effects for three different brands of indirect $a$-Si EPIDs. These ghosting effects cause an under-response in EPID signal and hence in measured portal dose.

Chapter 7 describes the development of a model to predict quantitatively dose-volume histogram (DVH) parameter changes based on 2D transit dosimetry. The model has been implemented for conventional breast treatments where large dose differences may occur due to the presence of set-up errors. These differences have been traced by combining all methods and results from chapters 4-6 into a clinical 2D transit dosimetry procedure that was used for all breast patients treated in our department 
with curative intent. The accuracy of the prediction model has been tested by applying it to clinically obtained results from this procedure.

\section{Discussion}

Finally, in Chapter 8 the results described in this thesis, their clinical implications and future perspectives are discussed.

Because the methods were implemented in clinical practice, a technical infrastructure for handling and analyzing vast amounts of images had to be designed and implemented, which is presented in the Appendix. 


\section{REFERENCES}

1 J. Ferlay, P. Autier, M. Boniol, M. Heanue, M. Colombet, and P. Boyle, "Estimates of the cancer incidence and mortality in Europe in 2006," Ann. Oncol. 18, 581-592 (2007).

2 A. Jemal, R. Siegel, E. Ward, T. Murray, J. Xu, and M.J. Thun, "Cancer Statistics," CA. Cancer. J. Clin. 57, 43-66 (2007).

3 S.M. Bentzen, G. Heeren, B. Cottier, B. Slotman, and B. Glimelius, "Towards evidence-based guidelines for radiotherapy infrastructure and staffing needs in Europe: the ESTRO QUARTS project," Radiother. Oncol. 75, 355-365 (2005).

4 P.M. Lapostolle, "Introduction to RF linear accelerators (lineacs)," Proceedings of the CERN Accelerator School: cyclotrons, linacs and their applications 1-16 (1996).

5 D.J. Warner, "Fundamentals of electron linacs," Proceedings of the CERN Accelerator School: cyclotrons, linacs and their applications 17-38 (1996).

6 M. Weiss, "Matching accelerators to the task," Proceedings of the CERN Accelerator School: cyclotrons, linacs and their applications 381-394 (1996).

7 A.L. Boyer, L. Antonuk, A. Fenster, M. Van Herk, H. Meertens, P. Munro, L.E. Reinstein, and J. Wong, "A review of electronic portal imaging devices (EPIDs)," Med. Phys. 19, 1-16 (1992).

8 L.E. Antonuk, "Electronic portal imaging devices: a review and historical perspective of contemporary technologies and research," Phys. Med. Biol. 47, R31-65 (2002).

9 B. Fraass, K. Doppke, M. Hunt, G. Kutcher, G. Starkschall, R. Stern, and J. Van Dyke, "American Association of Physicists in Medicine Radiation Therapy Committee Task Group 53: quality assurance for clinical radiotherapy treatment planning," Med. Phys. 25, 1773-1829 (1998).

10 B.J. Mijnheer, J.J. Battermann, and A. Wambersie, "What degree of accuracy is required and can be achieved in photon and neutron therapy?" Radiother. Oncol. 8, 237-252 (1987).

11 C.A. McBain, A.M. Henry, J. Sykes, A. Amer, T. Marchant, C.M. Moore, J. Davies, J. Stratford, C. McCarthy, B. Porritt, P. Williams, V.S. Khoo, and P. Price, "X-ray volumetric imaging in image-guided radiotherapy: the new standard in on-treatment imaging," Int. J. Radiat. Oncol. Biol. Phys. 64, 625-634 (2006).

12 O. Morin, A. Gillis, J. Chen, M. Aubin, M.K. Bucci, M. Roach, 3rd, and J. Pouliot, "Megavoltage cone-beam CT: system description and clinical applications," Med. Dosim. 31, 51-61 (2006).

13 C. Amies, A. Bani-Hashemi, J.C. Celi, G. Grousset, F. Ghelmansarai, D. Hristov, D. Lane, M. Mitschke, A. Singh, H. Shukla, J. Stein, and M. Wofford, "A multi-platform approach to image guided radiation therapy (IGRT)," Med. Dosim. 31, 12-19 (2006).

14 D. Verellen, M.D. Ridder, N. Linthout, K. Tournel, G. Soete, and G. Storme, "Innovations in image-guided radiotherapy," Nat. Rev. Cancer. 7, 949-960 (2007).

15 J. van Dam and G. Marinello, Methods for in vivo dosimetry in external radiotherapy. ESTRO Booklet No. 1. 1994.

16 E. Yorke, R. Alecu, L. Ding, D. Fontenla, A. Kalend, D. Kaurin, M.E. Masterson-McGary, G. Marinello, T. Matzen, A. Saini, J. Shi, W. Simon, T.C. Zhu, and X.R. Zhu, Diode in vivo dosimetry for patients receiving external beam radiation therapy. AAPM Report No. 87. 2005.

17 G. Bharanidharan, D. Manigandan, K. Devan, V. Subramani, N. Gopishankar, T. Ganesh, R. Joshi, G. Rath, J. Velmurugan, P. Aruna, and S. Ganesan, "Characterization of responses and comparison of calibration factor for commercial MOSFET detectors," Med. Dosim. 30, 213-218 (2005). 
18 E.J. Bloemen-van Gurp, A.W. Minken, B.J. Mijnheer, C.J. Dehing-Oberye, and P. Lambin, "Clinical implementation of MOSFET detectors for dosimetry in electron beams," Radiother. Oncol. 80, 288-295 (2006).

19 C. Fiorino, D. Corletto, P. Mangili, S. Broggi, A. Bonini, G.M. Cattaneo, R. Parisi, A. Rosso, P. Signorotto, E. Villa, and R. Calandrino, "Quality assurance by systematic in vivo dosimetry: results on a large cohort of patients," Radiother. Oncol. 56, 85-95 (2000).

20 M. Essers, R. Keus, J.H. Lanson, and B.J. Mijnheer, "Dosimetric control of conformal treatment of parotid gland tumours," Radiother. Oncol. 32, 154-162 (1994).

21 E. Bloemen-van Gurp, W. du Bois, P. Visser, I. Bruinvis, D. Jalink, J. Hermans, and P. Lambin, "Clinical dosimetry with MOSFET dosimeters to determine the dose along the field junction in a split beam technique," Radiother. Oncol. 67, 351-357 (2003).

22 A. Niroomand-Rad, C.R. Blackwell, B.M. Coursey, K.P. Gall, J.M. Galvin, W.L. McLaughlin, A.S. Meigooni, R. Nath, J.E. Rodgers, and C.G. Soares, "Radiochromic film dosimetry: Recommendations of AAPM Radiation Therapy Committee Task Group 55," Med. Phys. 25, 2093-2115 (1998).

23 M. Alber, S. Broggi, C. De Wagter, I. Eichwurzel, P. Engstrom, C. Fiorino, D. Georg, G. Hartmann, T. Knoos, A. Leal, H. Marijnissen, B. Mijnheer, M. Paiusco, F. Sanchez-Doblado, R. Schmidt, M. Tomsej, and H. Welleweerd, Guidelines for the verification of IMRT. ESTRO Booklet No. 9. 2008.

24 H. Gustavsson, A. Karlsson, S.A. Back, L.E. Olsson, P. Haraldsson, P. Engstrom, and H. Nystrom, "MAGIC-type polymer gel for three-dimensional dosimetry: intensity-modulated radiation therapy verification," Med. Phys. 30, 1264-1271 (2003).

25 K. Vergote, Y. De Deene, F. Claus, W. De Gersem, B. Van Duyse, L. Paelinck, E. Achten, W. De Neve, and C. De Wagter, "Application of monomer/polymer gel dosimetry to study the effects of tissue inhomogeneities on intensity-modulated radiation therapy (IMRT) dose distributions," Radiother. Oncol. 67, 119-128 (2003).

26 W.J.C. van Elmpt, L.N. McDermott, S.M.J.J.G. Nijsten, M. Wendling, P. Lambin, and B.J. Mijnheer, "A literature review of electronic portal imaging for radiotherapy dosimetry," Radiother. Oncol. 88, 289-309 (2008).

27 V.N. Hansen, P.M. Evans, and W. Swindell, "The application of transit dosimetry to precision radiotherapy," Med. Phys. 23, 713-721 (1996).

28 J. Pouliot, "Megavoltage imaging, megavoltage cone beam CT and dose-guided radiation therapy," Front. Radiat. Ther. Oncol. 40, 132-142 (2007).

29 W.J.C. van Elmpt, S.M.J.J.G. Nijsten, A.L.A.J. Dekker, B.J. Mijnheer, and P. Lambin, "Treatment verification in the presence of inhomogeneities using EPID-based threedimensional dose reconstruction," Med. Phys. 34, 2816-2826 (2007).

30 A. Noel, "Verification of the dose delivered to the patient by means of TLD, SC, PID. What future?," Cancer. Radiother. 7 Suppl. 1, s78-84 (2003). 


\title{
INTRODUCTION
}

\author{
POINT DOSE VERIFICATION \\ METHODS AND APPLICATION
}

TWO-DIMENSIONAL TRANSIT DOSIMETRY METHODS AND APPLICATION

DISCUSSION 



\section{CHAPTER}

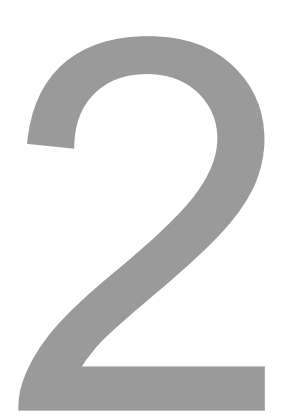

Verification of treatment parameter transfer by means of electronic portal dosimetry

Med. Phys. 31(2), 341-347 (2004)

S.M.J.J.G. Nijsten

A.W.H. Minken

P. Lambin I.A.D. Bruinvis 



\section{ABSTRACT}

Electronic portal imaging devices (EPIDs) are mainly used for patient set-up verification during treatment but other geometric properties like block shape and leaf positions are also determined. Electronic portal dosimetry allows dosimetric treatment verification. By combining geometric and dosimetric information, the data transfer between treatment planning system (TPS) and linear accelerator can be verified which in particular is important when this transfer is not carried out electronically. We have developed a pre-treatment verification procedure of geometric and dosimetric treatment parameters of a $10 \mathrm{MV}$ photon beam using an EPID. Measurements were performed with a CCD camera-based $i$ View EPID, calibrated to convert a grayscale EPID image into a two-dimensional absolute dose distribution. Central field dose calculations, independent of the TPS, are made to predict dose values at a focus-EPID distance of $157.5 \mathrm{~cm}$. In the same EPID image, the presence of a wedge, its direction, and the field size defined by the collimating jaws were determined. The accuracy of the procedure was determined for open and wedged fields for various field sizes. lonization chamber measurements were performed to determine the accuracy of the dose values measured with the EPID and calculated by the central field dose calculation. The mean difference between ionization chamber and EPID dose at the center of the fields was $0.8 \pm 1.2 \%$ ( $1 \mathrm{SD}$ ). Deviations larger than $2.5 \%$ were found for half fields and fields with a jaw in overtravel. The mean difference between ionization chamber results and the independent dose calculation was $-0.2 \pm 0.6 \%$ ( $1 \mathrm{SD}$ ). For all wedged fields, the presence of the wedge was detected and the mean difference in actual and measured wedge direction was $0 \pm 3^{\circ}$ (1 SD). The mean field size differences in $X$ and $Y$ directions were $0.1 \pm 0.1 \mathrm{~cm}$ and $0.0 \pm 0.1 \mathrm{~cm}(1 \mathrm{SD})$, respectively. Pre-treatment monitor unit verification is possible with high accuracy and also geometric parameters can be verified using the same EPID image. 


\subsection{INTRODUCTION}

Electronic portal imaging devices (EPIDs) are widely used to monitor patient position during daily radiotherapy sessions. Several on- and off-line verification procotols have been developed for this purpose. ${ }^{1,2}$ EPID images can also be used for geometric applications other than measuring patient set-up. Luchka et al. ${ }^{3}$ showed, for instance, that it is possible to assess radiation and light field congruence; Partridge et $a l^{4,5}$ implemented a method to verify individual leaf motion during an intensity-modulated beam delivery by dynamic multileaf collimator techniques. Besides geometric verification, portal imaging can also be used for dosimetric verification: electronic portal dosimetry (EPD).

Several methods have been described to extract dose information from a grayscale EPID image. Heijmen et al. ${ }^{6}$ and Pasma et al. ${ }^{7}$ modeled the response of a low-elbow video-based SRI-100 EPID (Philips, The Netherlands) to obtain absolute transmission dose distributions. For open, wedged, and intensity-modulated radiation therapy (IMRT) using $25 \mathrm{MV}$ photon beams, EPID and ionization chamber measurements agreed to within 1\% (1 SD) for a set of anthropomorphic phantoms for symmetric fields. De Boer et $a l^{8}{ }^{8}$ described the dosimetric calibration needed to measure transmission dose with a high-elbow video-based Theraview-NT EPID (Cablon Medical, The Netherlands). Several authors reported on the use of a scanning liquid-filled EPID for dose measurements. ${ }^{9-12}$ Portal dose images are frequently used for (1) portal in vivo dosimetry and (2) pre-treatment dose verification of IMRT fields.

In case of portal in vivo dosimetry, the aim is to verify the dose actually delivered to a patient during treatment. In general, two approaches are used. In first approach, the measured portal dose is back-projected to the patient to obtain the exit, midplane, or reconstructed patient dose, and consequently is compared with the dose (distribution) of the treatment planning system (TPS). ${ }^{13-18}$ In second approach, a portal dose is calculated which can be directly compared with the measured portal dose information. According to Pasma et al., ${ }^{19,20}$ good agreement is found between calculated and measured transmission dose distributions behind anthropomorphic phantoms (differences less than 1\%) using an equivalent homogeneous phantom made of polystyrene and a set of measured transmission functions for high energy photon beams.

In case of pre-treatment dose verification of IMRT fields, the aim is to verify the accuracy of dose delivery in a phantom prior to the first radiotherapy session. Van Esch et al. $^{21}$ and Pasma et $a .^{22}$ described, for instance, the accuracy of pre-treatment verification of IMRT fields produced with dynamic multileaf collimation. They acquired portal dose images with a liquid-filled and a video-based EPID, respectively. The dosimetric accuracy of the measured portal dose images was $2 \%$ (1 SD) with respect to film and ionization chamber measurements. The use of an algorithm based on pencil beam kernels ${ }^{23,24}$ was proposed to calculate dose images, which reflect the intended transmission dose distributions at the large focus-EPID distance in absence of a patient. 
For two-dimensional (2D) portal dose verification with and without patient, a dose calculation algorithm is required which accurately predicts portal dose images at large focus-EPID distances. An important drawback of such a procedure is that these dose calculation algorithms (like the ones above) are not yet available in all commercial treatment planning systems.

Therefore, it was the purpose of our investigation to develop a pre-treatment verification procedure based on EPD to verify a portal dose at the center of open and wedged fields without patient, avoiding large measurement sets needed for 2D portal dose calculation. Besides verification of monitor units (MUs), the same EPID image was used to verify geometrical beam properties allowing us to investigate the feasibility of using only one EPID image for verification of treatment parameter transfer. This is especially important when data transfer between TPS and linear accelerator is not carried out electronically.

\subsection{MATERIALS AND METHODS}

\subsubsection{Equipment}

The EPID used in this work was a video-based iView EPID (Elekta, Crawley, United Kingdom) consisting of a fluorescent screen, two mirrors, and a CCD camera (KP-M1E/K-S10, Hitachi, Santa Clara, CA) which is read out to obtain a digital portal image. The sensitive layer of this 8 bit low-elbow EPID has a fixed distance of $160 \mathrm{~cm}$ from the accelerator focus and an effective field-of-view (FOV) of $19 \mathrm{~cm} \times 25 \mathrm{~cm}$. The FOV and all field sizes, coordinates, and distances to the collimator rotation axis are defined in the plane through the isocenter, at $100 \mathrm{~cm}$ from the focus. The number of pixels in an image was $576 \times 768$ with a maximum number of grayscale values of 256 . The pixel resolution was approximately $0.3 \mathrm{~mm}$ after resampling the images to obtain square pixels.

The EPID was mounted to a SL20 linear accelerator (Elekta, Crawley, United Kingdom), which is equipped with a motorized internal wedge, fixed to the collimator. The wedge can only be moved in or out the treatment field completely and has a fixed wedge angle of $60^{\circ}$. A wedge angle smaller than $60^{\circ}$ can be achieved during irradiation by applying open and wedged beams separately but in this work only the maximum wedge angle of $60^{\circ}$ was used. The linear accelerator is equipped with two pairs of collimating jaws, which are positioned at different distances to the accelerator focus and can move independently from each other. All measurements were performed in a $10 \mathrm{MV}$ photon beam with a nominal dose rate of approximately $550 \mathrm{MU} \cdot \mathrm{min}^{-1}$. No shielding by blocks or multileaf collimator was done.

\subsubsection{Dosimetric calibration of the EPID}

Dosimetric calibration of the iView EPID was performed to convert a grayscale EPID image into a two-dimensional (absolute) dose distribution in the plane perpendicular 
to the collimator rotation axis for fixed values of focus-surface distance $f_{R}$ and depth $z_{R}$ in water. In this work, the values of $f_{R}$ and $z_{R}$ are 157.5 and $2.5 \mathrm{~cm}$, respectively; this depth is slightly larger than the depth of dose maximum at $10 \mathrm{MV}$ to reduce electron contamination effects.

To decrease the influence of high energetic contaminating electrons to the EPID dose measurements, an additional sheet of 1-mm-thick stainless steel was placed on top of the 1.65-mm-thick stainless steel plate already present in the EPID. The layer of 2.65 $\mathrm{mm}$ steel is equivalent to approximately $2.5 \mathrm{~cm}$ water.

Heijmen et al. ${ }^{6}$ described the excellent suitability of the SRI-100 EPID, the predecessor of the iView EPID, for portal dosimetry. Because both EPIDs are CCD camera-based with no differences in EPID box and optical components, reproducibility and stability are the same.

In the initialization software of the iView EPID several parameters were changed: (1) the black level voltage (camera offset) was chosen such that the average grayscale value in an EPID image acquired without radiation was approximately 20 . This was done to avoid truncation errors by negative "dark current" signals; (2) the white level voltage was selected to obtain grayscale values of 230 in a single frame for an irradiation with an open $30 \mathrm{~cm} \times 30 \mathrm{~cm}$ field. By using an on-axis grayscale value of 230 , off-axis dose variations up to $10 \%$ can be measured for this large field. In clinical practice, field sizes and hence output factors are usually smaller avoiding pixel saturation; (3) the flood field correction in the iView software was disabled by replacing the flood field images at four gantry angles by "unity" images containing only one pixel value inside the acquisition FOV. Outside this FOV, pixel values were set to zero for edge detection purposes; (4) a fixed integration time of $230 \mathrm{~ms}$ was used to obtain grayscale values of 230 for an open $30 \mathrm{~cm} \times 30 \mathrm{~cm}$ field in a single frame ensuring a high signal-to-noise ratio (SNR) without getting pixel saturation. The integration time has to be determined independently from the white level voltage for the View EPID; (5) the number of frames was optimized to acquire images over a fixed time interval of approximately $30 \mathrm{~s}$ and was chosen equal to 105 frames. The accumulation and read-out of an image frame introduces a dead time of approximately 80 ms which is not further taken into account in this work.

The response of the EPID was corrected for (1) optical crosstalk in the EPID box and (2) non-uniformity in fluorescent screen and CCD elements, according to Heijmen et al. ${ }^{6}$ and Pasma et al. ${ }^{7}$ Based on measurements, an optical crosstalk kernel and EPID sensitivity matrices were derived to convert a grayscale EPID image into a 2D portal dose distribution numerically.

Dose measurements for the optical crosstalk correction were performed with a Baldwin-Farmer ionization chamber $\left(0.6 \mathrm{~cm}^{3}\right)$ in a water phantom the collimator rotation axis. Square field sizes of $3 \mathrm{~cm} \times 3 \mathrm{~cm}$ to $18 \mathrm{~cm} \times 18 \mathrm{~cm}$ were used. In the original publication of Pasma et al., ${ }^{7}$ dose measurements with a miniphantom were performed, allowing direct measurement of optical crosstalk in the EPID box. We measured dose in full scatter conditions and hence a field size correction was determined instead of a pure optical crosstalk correction. Optical crosstalk is 
inherently corrected by this field size correction however, and therefore this correction is still called optical crosstalk correction in this work. Relative dose measurements for the non-uniformity correction were performed with a scanning diode-array (Schuster $\mathrm{GmbH}$, Forchheim, Germany). This device consists of a linear array of 80 diodes and was calibrated to account for different sensitivities of the array elements at the EPID plane. Because of the long measurement time of 2-3 min, output variations of the linear accelerator were monitored by a small diode on top of a collimating jaw in the accelerator head within the blocked radiation field. Field sizes of $3 \mathrm{~cm} \times 3 \mathrm{~cm}$ to $18 \mathrm{~cm}$ $x 18 \mathrm{~cm}$ were used, resulting in several EPID sensitivity matrices. These matrices were applied for correcting changes in local EPID sensitivity but also to account for shortcomings of the optical crosstalk correction for off-axis points in an EPID image. ${ }^{7}$ All dose measurements and EPID measurements were done twice and were performed sequentially; the number of MUs was 300.

Finally, a two-dimensional relative dose matrix was converted into absolute dose values by means of one conversion factor equal to the ratio of the mean grayscale value within a FOV of $0.5 \mathrm{~cm} \times 0.5 \mathrm{~cm}$ and the absolute dose value at the collimator rotation axis of a $10 \mathrm{~cm} \times 10 \mathrm{~cm}$ field. An extra correction factor is used for wedged fields to correct for a non-linear response of the EPID to a different photon energy spectrum; this correction factor is multiplied with the absolute dose values. The number of pixels in an EPID dose matrix was $128 \times 128$ and the pixel resolution was approximately $2 \mathrm{~mm}$.

\subsubsection{Independent central field dose calculation}

In order to verify pre-treatment dose delivery using measurements with the EPID, an absolute dose value $D_{c, P L A N}$ was predicted at the geometrical center of a radiation field (central beam axis) for both open and wedged fields. In this work, an independent dose calculation algorithm is used because it is not possible to predict portal dose values with our treatment planning system FOCUS 3.0 (Computerized Medical Systems, St. Louis, MO).

The central field dose $D_{C, P L A N}$ in symmetric open and wedged fields with field sizes $c$ is expressed as

$$
D_{c, P L A N}(c, w)=\dot{D}_{R}\left(c_{R}\right) \cdot U \cdot O_{R}(c, w) \cdot k_{w}\left(c_{R}, w\right)
$$

where $\dot{D}_{R}$ is the absorbed dose per monitor unit, $U$ is the number of monitor units, $O_{R}$ is the output ratio for equivalent square field size $c$ relative to reference field size $c_{R}$ $(10 \mathrm{~cm} \times 10 \mathrm{~cm})$, and $k_{w}$ is the wedge factor. These parameters are all determined in a water phantom in full scatter conditions for the fixed values of focus-surface distance $f_{R}$ and depth $z_{R} ; \dot{D}_{R}$ and $k_{w}$ are determined for field size $c_{R}$. The symbol $w$ indicates the presence of a wedge in the beam.

Equation (2.1) originates from ESTRO Booklet No. $3^{25}$ and is simplified at several points: (1) a tissue-phantom ratio is not required to calculate dose values at depths other than $z_{R}$ : the EPID was calibrated for dose measurements at one fixed depth; (2) the 
equivalent square field size $c$ is used, neglecting differences in $O_{R}$ due to the collimator exchange effect.

Dose measurements were performed with a Baldwin-Farmer ionization chamber in a water phantom at $f_{R}$ and $z_{R}$ for field sizes varying from $3 \mathrm{~cm} \times 3 \mathrm{~cm}$ to $18 \mathrm{~cm} \times 18 \mathrm{~cm}$ to determine the output ratio $O_{R}(c, w)$ and the wedge factor $k_{w}\left(c_{R}, w\right)$. For symmetric wedged fields, the ionization chamber was located at the collimator rotation axis by minimizing the difference in chamber signal for measurements with the collimator rotated over $180^{\circ}$.

In case of asymmetric fields, Eq. (2.1) has been generalized for off-axis points by introducing off-axis correction factors. ${ }^{26}$ To get the dose at the central beam axis for asymmetric fields, the dose at the collimator rotation axis for equivalent square field size $c$ is multiplied with the off-axis factors. Therefore, if the beam axis is at $(x, y)$ position from the collimator axis:

$$
D_{c, P L A N}(c, x, y, w)=D_{c, P L A N}(c, w) \cdot A(c, x, w) \cdot A(c, y, w)
$$

where $A(c, x, w)$ and $A(c, y, w)$ are the off-axis factors in the wedge direction and perpendicular to the wedge direction, respectively, for fixed $f_{R}$ and $z_{R}$. The off-axis factors are determined experimentally for asymmetric wedged fields only. In case of asymmetric open fields, the off-axis factors were set to 1 because beam profiles are relatively flat at the large focus-surface distance $f_{R}$.

The factors $A(c, x, w)$ and $A(c, y, w)$ were determined for field sizes of $5 \mathrm{~cm} \times 5 \mathrm{~cm}, 10 \mathrm{~cm}$ $x 10 \mathrm{~cm}$, and $15 \mathrm{~cm} \times 15 \mathrm{~cm}$; the position of the central beam axis was varied between 25 and $15 \mathrm{~cm}$ in the wedge direction ( $X$ direction) and from 0 to $7.5 \mathrm{~cm}$ perpendicular to the wedge direction ( $Y$ direction). A step size of $2.5 \mathrm{~cm}$ was used. Again, all measurements were done with the Baldwin-Farmer ionization chamber in a water phantom at $f_{R}$ and $z_{R}$. The long axis of the Baldwin-Farmer ionization chamber was oriented perpendicular to the wedge direction.

\subsubsection{Pre-treatment verification procedure}

The ultimate goal of quality assurance in radiotherapy is to verify whether the dose distribution determined with the treatment planning system is equal to the dose delivered to the patient. In absence of a patient, only the transfer of MUs between TPS and linear accelerator can be verified by EPD measurements, which is the approach chosen in this work. Performing a pre-treatment verification procedure without a patient means an extra measurement but in this way possible large errors can be detected before the actual patient treatment starts. Similar measurements can be performed by placing dosimeters on the skin of the patient during treatment (i.e., entrance dose measurements). In this way also the source-skin-distance can be verified, but the measurement only offers one-dimensional information and does not allow verification of geometric treatment parameters like the EPID inherently does. 
With our verification procedure, four treatment field properties are determined in a portal dose image: (1) the central field dose, (2) the presence of a wedge, (3) the wedge direction, and (4) the field size defined by the collimating jaws.

The central field dose $D_{c, E P I D}$ is equal to the dose values averaged over a region-of-interest of $0.6 \mathrm{~cm} \times 0.6 \mathrm{~cm}$ and is determined at the center of the radiation field. The position of the central beam axis in a portal dose image is calculated from collimator jaw settings and collimator angle from the treatment planning system, and sagging information for the EPID. The accuracy of the central field dose verification procedure was assessed by comparing both the central dose measured by the EPID, $D_{c, E P I D}$, and the central dose calculated with Eq. (2.2), $D_{C, P L A N}$, with the central dose measured by an ionization chamber, $D_{c, \text { CHAM }}$. A Baldwin-Farmer chamber was used in a water phantom at focus-skin distance $f_{R}$ and with its effective center at depth $z_{R}$ in water. Five groups of treatment fields were used: (1) square open fields, (2) square wedged fields, (3) rectangular open fields, (4) asymmetric open fields and (5) asymmetric wedged fields. Relative dose differences are reported as " $\left[D_{c, x} \mathrm{vs} D_{c, y}\right]$ " and are defined as $\left(D_{c, x}-D_{c, y}\right) \times 100 / D_{c, x}$. For each group of treatment fields, three mean dose difference values were calculated: $\left[D_{c, \text { CHAM }}\right.$ vs $\left.D_{c, \text { PLAN }}\right],\left[D_{c, \text { CHAM }}\right.$ vs $\left.D_{c, \text { EPID }}\right]$, and $\left[D_{c, \text { PLAN }}\right.$ vs $\left.D_{C, E P I D}\right]$.

Both open and wedged square fields had sizes which varied from $4 \mathrm{~cm} \times 4 \mathrm{~cm}$ up to 18 $\mathrm{cm} \times 18 \mathrm{~cm}$. In case of rectangular open fields, one field dimension was equal to 10 or $18 \mathrm{~cm}$ and the other varied from 4 to $10 \mathrm{~cm}$ or from 6 to $18 \mathrm{~cm}$, respectively. For asymmetric open fields, the position of the central beam axis moved to off-axis positions of 2.5 or $4.5 \mathrm{~cm}$ toward the "toe" of the wedge by changing the X2-jaw position, starting with field sizes of $10 \mathrm{~cm} \times 10 \mathrm{~cm}$ or $18 \mathrm{~cm} \times 18 \mathrm{~cm}$, respectively; identical measurements were performed by shifting the central beam axis toward the "heel" of the wedge by varying the X1-jaw position. In the $Y$ direction, which is perpendicular to the wedge direction, only the position of the $Y 2$ jaw is changed. The central beam axis was moved to off-axis positions of 2.5 or $7 \mathrm{~cm}$ for initial field sizes of $10 \mathrm{~cm} \times 10 \mathrm{~cm}$ or $18 \mathrm{~cm} \times 18 \mathrm{~cm}$, respectively. Asymmetric wedged fields were measured according to the same procedure as used for the asymmetric open fields. An initial field size of $16 \mathrm{~cm} \times 18 \mathrm{~cm}$ was used instead of $18 \mathrm{~cm} \times 18 \mathrm{~cm}$ giving a maximum central beam axis movement of $4.0 \mathrm{~cm}$ in the $X$ direction. Both asymmetric open and wedged fields were asymmetric in one direction only.

The presence of a wedge in a radiation beam causes an intensity variation in one direction of an EPID image. To determine whether a beam is wedged or not, a coordinate system is defined at the center of the image and dose values are determined relative to the origin of the system in two orthogonal directions. In each direction the ratio of the dose values on both sides of the origin is calculated. If at least one of the two ratios is larger than 1.05, it is concluded that a wedge was present in the beam. To avoid false positive results, the points taken to measure the dose have to be positioned at sufficient distance from the field edges (minimal $1 \mathrm{~cm}$ ). The distance of the points relative to the origin was $1 \mathrm{~cm}$ which allowed wedge detection for a minimum field size of $3 \mathrm{~cm} \times 3 \mathrm{~cm}$. The presence of a wedge was verified for all treatment fields. 
The wedge direction can also be derived from an EPID image. The wedge has a fixed orientation in the accelerator head for Elekta machines, thus the wedge direction is determined by the collimator angle. Around the origin of the coordinate system, a circle is drawn in the EPID image. Theoretically, it can be shown that the dose variation along the circumference of the circle has a sinusoidal shape with maximum amplitude at an angle equal to the collimator angle. In practice, the maximum value of the dose intensity profile along the circle circumference may not always give the proper collimator angle due to image noise and finite pixel size. Therefore a sine function is fitted to the dose profile and the maximum of this function is determined. Increasing the radius of the circle would improve the accuracy of the sine fit and hence the accuracy of the algorithm. In this work, a radius of $1 \mathrm{~cm}$ is used allowing wedge direction determination for small field sizes. The wedge direction was verified for a $10 \mathrm{~cm} \times 10 \mathrm{~cm}$ field with collimator angles varying between 0 and $360^{\circ}$ in steps of $40^{\circ}$ to investigate the accuracy of the wedge direction algorithm.

The field size defined by the four collimating jaws is determined by looking for the largest field dimensions along the two orthogonal axes in the EPID image for both open and wedged fields. The $50 \%$ dose value is determined by setting the dose at the center of the treatment field equal to $100 \%$ and the dose outside the field equal to $0 \%$. In horizontal and vertical directions of the EPID image, the most outer pixels of the treatment field with a dose value equal to or larger than the $50 \%$ dose value are marked. If these four "outer" pixels have 4 or more marked neighboring pixels, the distances between the pixel positions and the isocenter position in the EPID image are defined as the largest field dimensions in the two orthogonal directions. By using the collimator angle from the treatment planning system, the field sizes $X$ and $Y$ (defined by the $X$ pair and $Y$ pair of collimating jaws, respectively) are calculated. Field sizes were verified for all treatment fields.

\subsection{RESULTS}

\subsubsection{Central field dose verification}

For the square open and square wedged fields, the mean dose differences were very small. Both the EPID dose measurements and the dose calculations agreed to within $0.7 \%$ (1 SD) with the ionization chamber measurements (Table 2.1). In case of the rectangular open fields, the mean dose differences were slightly higher. The EPID dose measurements showed a systematic underestimation of $0.8 \%$ compared to the dose measured with the ionization chamber (Table 2.1). For asymmetric open and asymmetric wedged fields, the accuracy in EPID dose measurement and dose calculation is shown in Fig. 2.1 and Table 2.1. Analysis of these results showed that the dose differences increase when the position of the central beam axis is shifted further off-axis (Fig. 2.2). Deviations larger than $2.5 \%$ were found for half fields and fields with a jaw traveled over-axis. No field size effect could be detected for the EPID dose measurements. The agreement between the dose calculation and the dose measurement with the ionization chamber is to within $0.9 \%$ (1 SD) deviation. A wedge 


\begin{tabular}{|c|c|c|c|c|c|c|}
\hline & \multicolumn{6}{|c|}{ Mean relative dose difference and standard deviation (\%) } \\
\hline & $\begin{array}{l}\text { Square } \\
\text { open } \\
\text { fields } \\
(n=9)\end{array}$ & $\begin{array}{l}\text { Square } \\
\text { wedged } \\
\text { fields } \\
(n=10)\end{array}$ & $\begin{array}{l}\text { Rectangular } \\
\text { open } \\
\text { fields } \\
(n=10)\end{array}$ & $\begin{array}{l}\text { Asym. } \\
\text { open } \\
\text { fields } \\
(n=14)\end{array}$ & $\begin{array}{l}\text { Asym. } \\
\text { wedged } \\
\text { fields } \\
(n=13)\end{array}$ & $\begin{array}{c}\text { All } \\
\text { fields } \\
(n=56)\end{array}$ \\
\hline$D_{c, \text { CHAM VS }} D_{c, \text { PLAN }}$ & $0.0 \pm 0.2$ & $-0.2 \pm 0.3$ & $-0.2 \pm 0.4$ & $-0.2 \pm 0.7$ & $-0.5 \pm 1.0$ & $-0.2 \pm 0.6$ \\
\hline$D_{c, \text { CHAM }}$ VS $D_{C, E P I D}$ & $0.1 \pm 0.2$ & $0.3 \pm 1.1$ & $0.8 \pm 0.3$ & $1.5 \pm 1.2$ & $0.8 \pm 1.8$ & $0.8 \pm 1.2$ \\
\hline$D_{C, P L A N}$ VS $D_{C, E P I D}$ & $0.1 \pm 0.2$ & $0.5 \pm 1.0$ & $1.0 \pm 0.4$ & $1.8 \pm 1.6$ & $1.3 \pm 2.5$ & $1.0 \pm 1.6$ \\
\hline
\end{tabular}

in the treatment field reduced the accuracy of a measurement not significantly compared to the asymmetric open fields. An overview of all dose verification results is given in Fig. 2.3 and Table 2.1.

\subsubsection{Geometric verification}

The presence of a wedge was detected in all 24 wedged fields. The accuracy of the wedge direction algorithm was investigated by determining the wedge direction for 10 different collimator angles. The best agreement between actual collimator angle and measured collimator angle was found by sampling the dose profile along the entire $360^{\circ}$ circle circumference in steps of 4 degrees. The mean difference in collimator angle was $0 \pm 3^{\circ}(1 \mathrm{SD})$. Verification of field size was done for all 56 fields which resulted in mean field size differences $\left[X_{P L A N}\right.$ VS $\left.X_{E P I D}\right]$ and $\left[Y_{P L A N}\right.$ VS $\left.Y_{E P I D}\right]$ of $0.1 \pm 0.1 \mathrm{~cm}$ and $0.0 \pm 0.1 \mathrm{~cm}(1 \mathrm{SD})$, respectively (Fig. 2.4).

\subsection{DISCUSSION}

\subsubsection{Central field dose verification}

In general, mean dose differences between EPID dose and ionization chamber dose exceed the differences between calculated dose and ionization chamber dose (Table 2.1). This means that dose differences $\left[D_{c, P L A N}\right.$ vs $\left.D_{c, E P I D}\right]$ are mainly caused by the accuracy of the EPID dose measurement. Hence, dose differences between EPID dose and ionization chamber dose can be explained by examining the dosimetric calibration of the iView EPID.

The dosimetric calibration of the iView EPID is based on square open fields, which explains the absence of dose differences for square fields. Although a separate conversion factor is used to transform the two-dimensional relative dose matrix into an absolute distribution for wedged fields, dose differences for square wedged fields are found up to $2 \%$ for other field sizes than $10 \mathrm{~cm} \times 10 \mathrm{~cm}$. This points out that the 


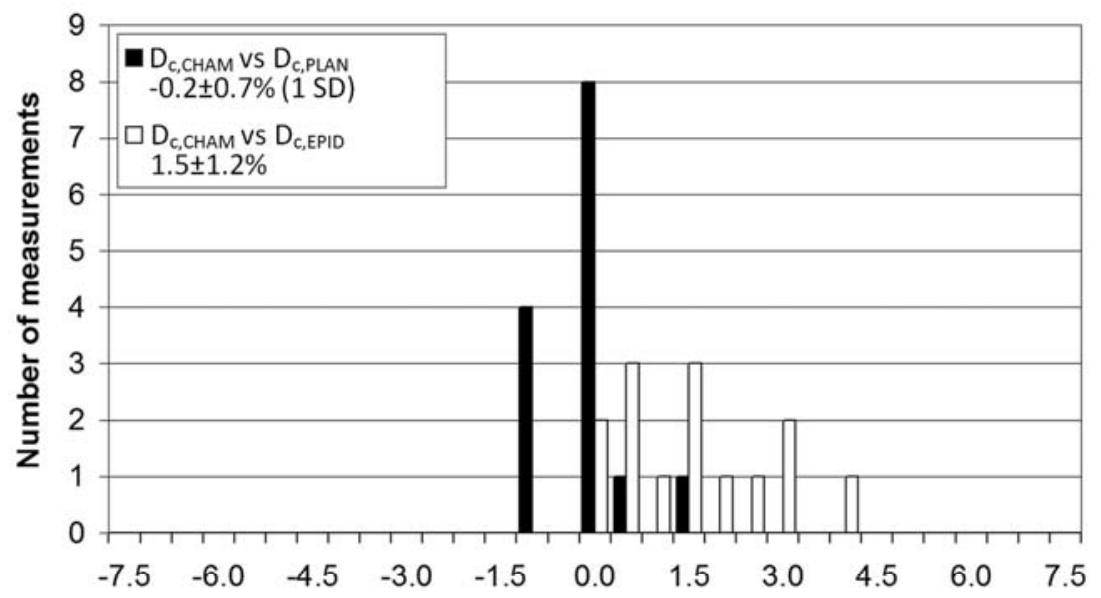

(a)

Dose difference (\%)

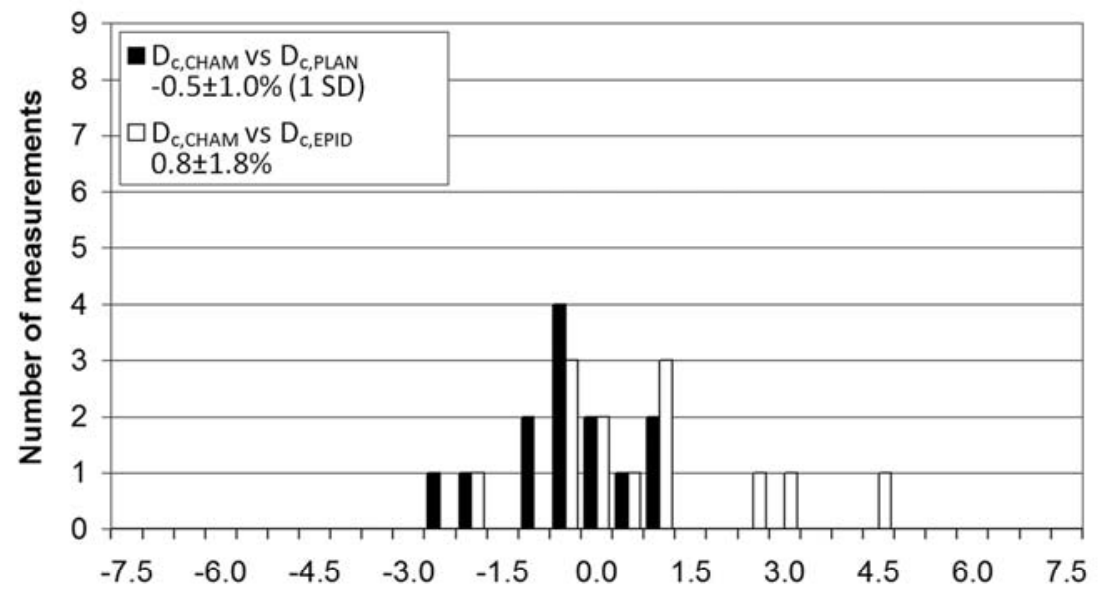

(b)

Dose difference (\%)

Figure 2.1: Relative dose differences between ionization chamber measurements, $D_{c, C H A M}$, EPID dose measurements, $D_{C, E P I D}$ and dose calculations, $D_{C, P L A N}$ for (a) 14 asymmetric open fields and (b) 13 asymmetric wedged fields.

conversion factor is slightly dependent on field size. In case of rectangular open fields, there is a systematic difference of $0.8 \%$ between EPID dose and ionization chamber dose values. This difference is still small and supports the use of the optical crosstalk correction for symmetric non-square fields. Heijmen et al. ${ }^{6}$ found a mean dose difference of only $0.2 \%$ with a standard deviation of $0.3 \%$ for comparable treatment fields measured with a SRI-100 EPID; the latter SD agrees perfectly with the SD we found. 


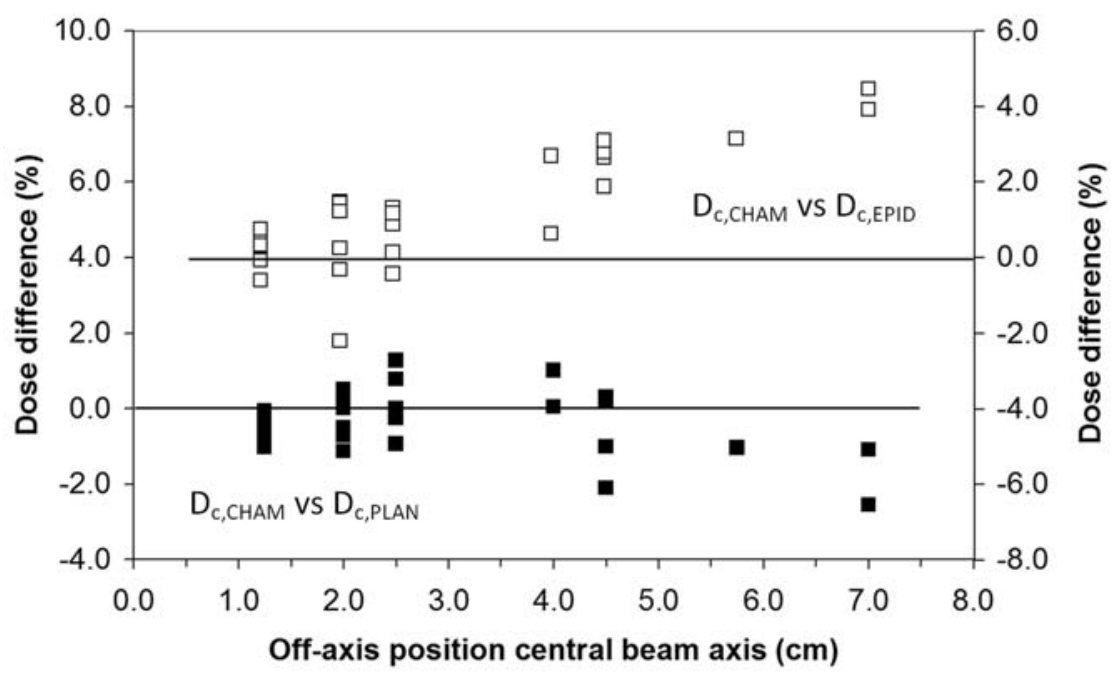

Figure 2.2: Relative dose differences between ionization chamber measurements and dose calculations (plotted on left $y$ axis), and between ionization chamber and EPID measurements (plotted on right $y$ axis) as a function of the off-axis position of the central beam axis in case of all 27 asymmetric open and wedged fields.

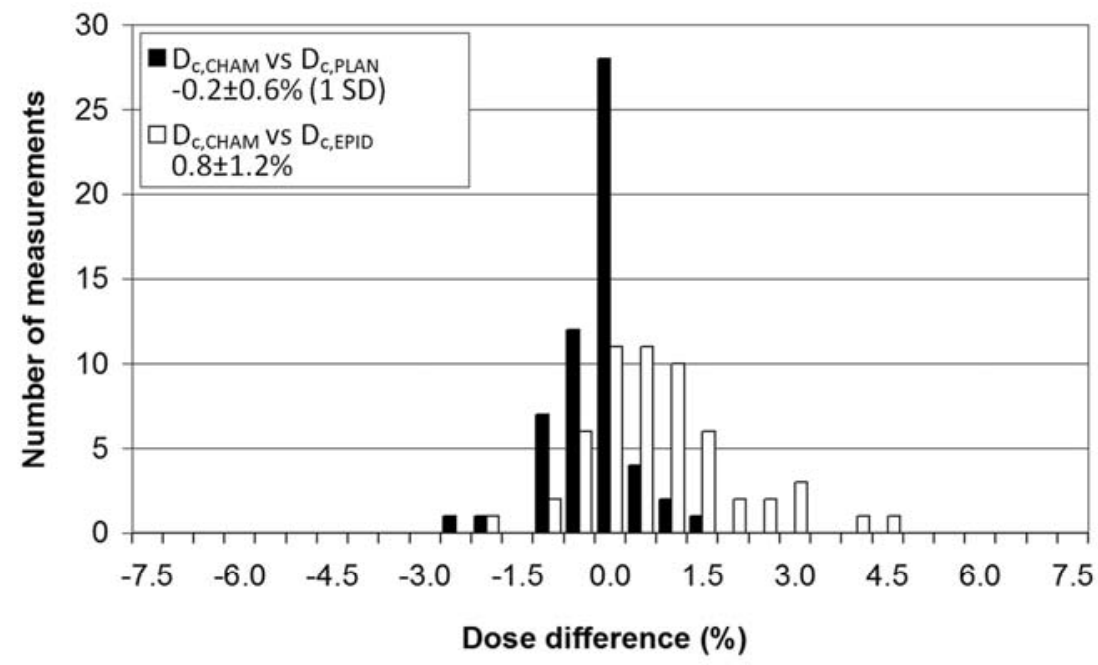

Figure 2.3: Relative dose differences for all 56 fields. 


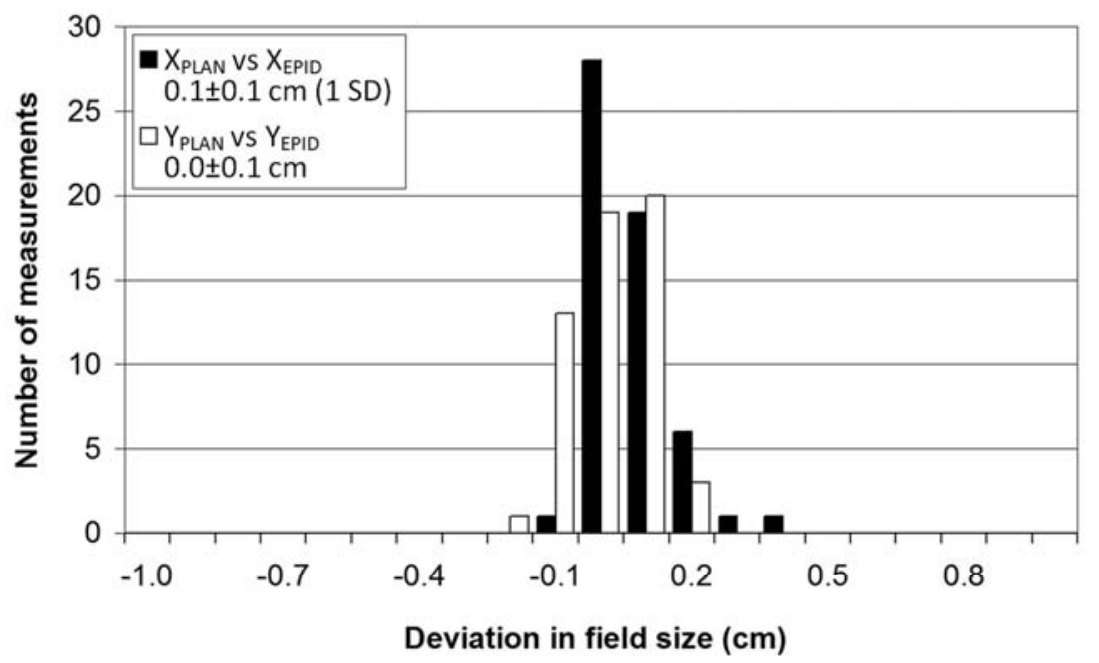

Figure 2.4: Field size differences between actual and measured field sizes in $X$ direction and $Y$ direction $\left[X, Y_{P L A N}\right.$ VS $\left.X, Y_{E P I D}\right]$ for all 56 fields.

Mean dose differences for both asymmetric open and wedged fields do no differ but the accuracy of EPID dose measurements is decreased with respect to the rectangular open fields. Furthermore, the magnitude of dose differences per field size is comparable for both open and wedged asymmetric fields $(p=0.1)$ and amounts up to $4.5 \%$ for a $16 \mathrm{~cm} \times 4 \mathrm{~cm}$ field with a central beam axis offset of $7 \mathrm{~cm}$. Differences between EPID dose and ionization chamber dose in case of off-axis points are caused by an incomplete crosstalk correction. In the optical crosstalk correction, invariance is assumed in both directions of an EPID image but this is not completely correct. For off-axis points close to the mirror in the low-elbow iView EPID, the EPID response increases with respect to reference dose data. ${ }^{7}$ Especially for off-axis points, the non-uniformity correction does not only take into account differences in sensitivity, but also the lack of invariance of the optical crosstalk correction. This means that for asymmetric fields, the accuracy of the non-uniformity correction decreases for off-axis dose measurements. The central beam axis shifts more off-axis when asymmetry increases causing a lower accuracy in EPID dose in both $X$ and $Y$ directions of the EPID. There might be two possible explanations for this observation. First, symmetric square field sizes are used during measurements for the non-uniformity correction resulting in symmetric square EPID sensitivity matrices. In case of the $16 \mathrm{~cm} \times 4 \mathrm{~cm}$ field with a central beam axis offset of $7 \mathrm{~cm}$, the sensitivity matrix of an $18 \mathrm{~cm} \times 18 \mathrm{~cm}$ field is used. Because EPID sensitivity matrices are field size dependent, the accuracy of EPID dose measurements will probably increase when (smaller) asymmetric field sizes are used for measuring the non-uniformity correction. Second, a Schuster diode array is used to perform the relative dose measurements needed for the non-uniformity correction. A Schuster diode array is not a full scatter instrument which implies that the EPID dose at off-axis points is not the dose under full scatter conditions either 
(opposed to the EPID dose measurements on the collimator rotation axis). To improve the accuracy of the off-axis EPID dose measurements, relative dose measurements have to be done under full scatter conditions (e.g., in a water phantom). However, with the existing non-uniformity correction used in this work, limiting the off-axis shift of the central beam axis can increase the accuracy of the EPID dose measurements. In case of the asymmetric open and wedged fields with an off-axis shift of the central beam axis smaller than $4 \mathrm{~cm},\left[D_{c, C H A M}\right.$ vs $\left.D_{c, E P I D}\right]$ is only $0.4 \pm 0.9 \%$ (1 SD) compared to $1.2 \pm 1.5 \%$ ( $1 \mathrm{SD}$ ) for all measured asymmetric fields.

In this work, a fixed wedge angle of $60^{\circ}$ is used for all measurements. Applying the pre-treatment procedure to a beam with a wedge angle smaller than $60^{\circ}$ means that two different dosimetric EPID images will be acquired and separately analyzed.

Differences between calculated dose and ionization chamber dose values are within 0.9\% (1 SD) for most field sizes. Only for asymmetric wedged fields, larger dose differences are found up to $2.6 \%$ for a $16 \mathrm{~cm} \times 4 \mathrm{~cm}$ field with a central beam axis offset of $7 \mathrm{~cm}$.

\subsubsection{Geometric verification}

In this work, the presence of a wedge and the wedge direction are determined on the central beam axis with excellent results. If the central beam axis is shielded by blocks or leaves, determination of the two geometric field properties will fail but analysis can still be performed in an unshielded part of the treatment field then. The accuracy of the wedge direction algorithm can be further improved by changing the radius of the circle in the EPID image and the number of sampling points of the dose profile along the circle circumference. Both parameters are dependent of the finite pixel sizes. Furthermore, analysis of more than one circle can also improve the accuracy because the signal-to-noise ratio will decrease. The field sizes are verified using a threshold intensity algorithm based on the $50 \%$ dose value marking the field edges. A gradient segmentation technique (e.g., based on Laplace operators) will be a more robust technique for detecting field edges or the exact field shaping by shielding blocks or a multileaf collimator.

\subsection{CONCLUSIONS}

We have developed a pre-treatment verification procedure based on electronic portal dosimetry, which can be used to verify treatment parameter transfer between TPS and linear accelerator. Both geometric and dosimetric parameters can be verified with a high accuracy using only one EPID image. After dosimetric calibration of an iView EPID, central field dose values can be measured with a relative accuracy of $1.2 \%$ (1 SD) for open and wedged fields. We showed that a 1D dose calculation algorithm can be used to accurately predict portal dose values to verify monitor unit transfer from TPS to linear accelerator. 
36 | Chapter 2

\section{ACKNOWLEDGEMENTS}

The authors would like to thank Ben Mijnheer for his helpful comments on the manuscript. 


\section{REFERENCES}

1 A. Bel, M.B. van Herk, H. Bartelink, and J.V. Lebesque, "A verification procedure to improve patient setup accuracy using portal imaging," Radiother. Oncol. 29, 253-260 (1993).

2 J.C.J. de Boer and B.J.M. Heijmen, "A protocol for the reduction of systematic patient setup errors with minimal portal imaging workload," Int. J. Radiat. Oncol., Biol., Phys. 50, 1350-1365 (2001).

3 K. Luchka, D. Chen, S. Shalev, G. Gluhchev, and R. Rajapakshe, "Assessing radiation and light field congruence with a video based electronic portal imaging device," Med. Phys. 21, 1245-1252 (1996).

4 M. Partridge, P.M. Evans, A. Mosleh-Shirazi, and D. Convery, "Independent verification using portal imaging of intensity-modulated beam delivery by the dynamic MLC technique," Med. Phys. 25, 1872-1879 (1998).

5 M. Partridge, J.R. Symonds-Tayler, and P.M. Evans, "IMRT verification with a camera-based electronic portal imaging system," Phys. Med. Biol. 45, 183-196 (2000).

6 B.J.M. Heijmen, K.L. Pasma, M. Kroonwijk, V.G.M. Althof, J.C.J. de Boer, A.G. Visser, and H. Huizenga, "Portal dose measurement in radiotherapy using an electronic portal imaging device," Phys. Med. Biol. 40, 1943-1955 (1995).

7 K.L. Pasma, M. Kroonwijk, J.C.J. de Boer, A.G. Visser, and B.J.M. Heijmen, "Accurate portal dose measurement with a fluoroscopic electronic portal imaging device for open and wedged beams and dynamic multileaf collimation," Phys. Med. Biol. 43, 2047-2060 (1998).

8 J.C.J. de Boer, B.J.M. Heijmen, K.L. Pasma, and A.G. Visser, "Characterization of a high-elbow, fluoroscopic electronic portal imaging device for portal dosimetry," Phys. Med. Biol. 45, 197-216 (2000).

9 R. Boellaard, M.B. van Herk, and B.J. Mijnheer, "The dose response relationship of a liquid-filled electronic portal imaging device," Med. Phys. 23, 1601-1611 (1996).

10 J. Chang, G.S. Mageras, C.C. Ling, and W. Lutz, "An iterative EPID calibration procedure for dosimetric verification that considers the EPID scattering factor," Med. Phys. 28, 2247-2257 (2001).

11 M. Essers, R. Boellaard, M.B. van Herk, H. Lanson, and B.J. Mijnheer, "Transmission dosimetry with a liquid-filled electronic portal imaging device," Int. J. Radiat. Oncol., Biol., Phys. 34, 931-941 (1996).

12 H. Parsaei and E. El-Khatib, "The use of an electronic portal imaging system to measure portal dose and portal dose profiles," Med. Phys. 25, 1903-1909 (1998).

13 R. Boellaard, M.B. van Herk, and B.J. Mijnheer, "A convolution model to convert transmission dose images to exit dose distributions," Med. Phys. 24, 189-199 (1997).

14 R. Boellaard, M.B. van Herk, H. Uiterwaal, and B.J. Mijnheer, "Twodimensional exit dosimetry using a liquid-filled electronic portal imaging device and a convolution model," Radiother. Oncol. 44, 149-157 (1997).

15 R. Boellaard, M. Essers, M.B. van Herk, and B.J. Mijnheer, "New method to obtain the midplane dose using portal in vivo dosimetry," Int. J. Radiat. Oncol., Biol., Phys. 41, 465-474 (1998).

16 V.N. Hansen, P.M. Evans, and W. Swindell, "The application of transit dosimetry to precision radiotherapy," Med. Phys. 23, 713-721 (1996).

17 T.R. McNutt, T.R. Mackie, P. Reckwerdt, and B.R. Paliwal, "Modeling dose distributions from portal dose images using the convolution/superposition method," Med. Phys. 23, 1381-1392 (1996).

18 X.G. Ying, L.Y. Geer, and J.W. Wong, "Portal dose images. II. Patient dose estimation," Int. J. Radiat. Oncol., Biol., Phys. 18, 1465-1475 (1990). 
19 K.L. Pasma, B.J.M. Heijmen, M. Kroonwijk, and A.G. Visser, "Portal dose image prediction for dosimetric treatment verification in radiotherapy. I. An algorithm for open beams," Med. Phys. 25, 830-840 (1998).

20 K.L. Pasma, S.C. Vieira, and B.J. Heijmen, "Portal dose image prediction for dosimetric treatment verification in radiotherapy. II. An algorithm for wedged beams," Med. Phys. 29, 925-931 (2002).

21 A. Van Esch, B. Vanstraelen, J. Verstraete, G. Kutcher, and D. Huyskens, "Pre-treatment dosimetric verification by means of a liquid-filled electronic portal imaging device during dynamic delivery of intensity modulated treatment fields," Radiother. Oncol. 60, 181-190 (2001).

22 K.L. Pasma, M.L.P. Dirkx, M. Kroonwijk, A.G. Visser, and B.J.M. Heijmen, "Dosimetric verification of intensity modulated beams produced with dynamic multileaf collimation using an electronic portal imaging device," Med. Phys. 26, 2373-2378 (1999).

23 P. Storchi and E. Woudstra, "Calculation models for determining the absorbed dose in water phantoms in off-axis planes of rectangular fields of open and wedged photon beams," Phys. Med. Biol. 40, 511-527 (1995).

24 P. Storchi and E. Woudstra, "Calculation of the absorbed dose distribution due to irregularly shaped photon beams using pencil beam kernels derived from basic input data," Phys. Med. Biol. 41, 637-656 (1996).

25 A. Dutreix, A. Bridier, B.E. Bjärngard, B.J. Mijnheer, J. Shaw, and H. Svensson, "Monitor Unit Calculation for High Energy Photon Beams," Garant, Apeldoorn, The Netherlands (1997).

26 B. Smulders, I.A. Bruinvis, and B.J. Mijnheer, "Monitor unit calculations for wedged asymmetric photon beams," Phys. Med. Biol. 47, 2013-2030 (2002). 


\section{CHAPTER}

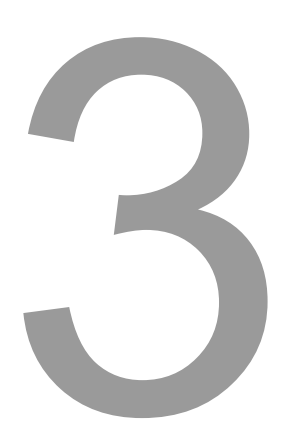

Routine individualized patient dosimetry using electronic portal imaging devices

Radiother. Oncol. 83(1), 65-75 (2007)

S.M.J.J.G. Nijsten

B.J. Mijnheer

A.L.A.J. Dekker

P. Lambin

A.W.H. Minken 



\section{ABSTRACT}

To analyze the results of routine EPID measurements for individualized patient dosimetry. Calibrated camera-based EPIDs were used to measure the central field dose, which was compared with a dose prediction at the EPID level. For transit dosimetry, dose data were calculated using patient transmission and scatter, and compared with measured values. Furthermore, measured transit dose data were back-projected to an in vivo dose value at $5 \mathrm{~cm}$ depth in water $\left(D_{5}\right)$ and directly compared with $D_{5}$ from the treatment planning system. Dose differences per treatment session were calculated by weighting dose values with the number of monitor units per beam. Reported errors were categorized and analyzed for approximately 37500 images from 2511 patients during a period of 24 months. Pre-treatment measurements showed a mean dose difference per treatment session of $0.0 \pm 1.7 \%$ ( $1 \mathrm{SD}$ ). Transfer errors were detected and corrected prior to the first treatment session. An accelerator output variation of about $4 \%$ was found between two weekly QC measurements. Patient dosimetry showed mean transit and $D_{5}$ dose differences of $-0.7 \pm 5.2 \%$ (1 SD) and $-0.3 \pm 5.6 \%$ (1 SD) per treatment session, respectively. Dose differences could be related to set-up errors, organ motion, erroneous density corrections and changes in patient anatomy. EPIDs can be used routinely to accurately verify treatment parameter transfer and machine output. By applying transit and in vivo dosimetry, more insight can be obtained with respect to the different error sources influencing dose delivery to a patient. 


\subsection{INTRODUCTION}

In high dose-high precision radiotherapy, treatment verification is necessary to assure the correct dose delivery to a patient. Several authors have proposed accuracy requirements. For instance, Mijnheer et al. ${ }^{1}$ proposed an accuracy requirement in absorbed dose delivery at the dose specification point of 3.5\% (1 SD) for external beam treatments based on the steepness of dose-effect curves for local tumor control and normal tissue damage. For specific high dose-high precision treatments this number may be decreased, or for less demanding situations increased. There are many potential error sources in a radiotherapy treatment, which can increase the uncertainty in the dose delivery to a patient to unacceptable values. Potential error sources can be subdivided into four categories ${ }^{2}:(1)$ errors in the data transfer from treatment planning system (TPS) to treatment equipment, (2) errors in the functioning of the treatment equipment, and errors that are patient related due to (3) set-up errors or organ motion and (4) inaccuracies during the treatment planning process. Various solutions can be chosen to detect and correct errors in these four categories but there are few solutions that can be utilized to detect errors in all four categories. Electronic portal imaging devices (EPIDs) can be used for this task because they are not only useful for imaging and position verification ${ }^{3-5}$ but are also suited for dosimetry ${ }^{6-7}$, both pre-treatment and during treatment.

In case of the first category, the data transfer between TPS and treatment equipment can be carried out electronically, avoiding an incorrect manual input of treatment parameters into the accelerator console. Although commercial solutions are widely available, even nowadays many radiotherapy departments are still using manual input which demands a verification procedure to verify geometric and dosimetric radiation parameters at the treatment machine. In case of electronic transfer, it could still be necessary to verify the treatment parameters because sometimes a "myriad" of computers is used in the chain between treatment planning and treatment delivery. Furthermore, manual input is sometimes necessary when not all treatment parameters are available during treatment planning (e.g. treatment couch position). Validating data transfer manually is perhaps most time-efficient but errors can be missed, as we were able to conclude from our own treatment error registration system. A pre-treatment verification method of geometric and dosimetric radiation parameters using an EPID has been developed in our institution and has been described elsewhere ${ }^{8}$. By using this method, the number of monitor units, as well as the presence of a wedge, its direction, and the field size are verified based on portal dose images, and hence the data transfer between TPS and treatment machine can be verified.

To avoid the second category of errors, routine quality assurance (QA) of treatment equipment is mandatory. Depending on the complexity of an irradiation technique, additive quality control can be performed. Intensity-modulated radiation therapy (IMRT) is a well-known example of an irradiation technique that demands a high accuracy of linear accelerator and multileaf collimator. IMRT QA programmes 
therefore pay a lot of attention to geometric verification in addition to dosimetric verification. Both can be performed using EPIDs ${ }^{9-11}$.

In case of the third and fourth category, errors in dose delivery are caused by set-up errors or organ motion and by inaccuracies during the treatment planning process, respectively. These patient related delivery errors can be found with semiconductor detectors and radiothermoluminescent dosimeters ${ }^{2,12}$ but also with portal imaging ${ }^{3-5}$ or portal dosimetry ${ }^{13-14}$. For instance, due to gas pockets inside the rectum, a different dose distribution can result inside a patient compared to the distribution predicted by the TPS $^{13}$. In case of inaccuracies in the dose calculation algorithm of the TPS, e.g. for tissue heterogeneities, the predicted three-dimensional (3D) dose distribution differs from the measured distribution ${ }^{14}$. To detect these errors with EPIDs, a back-projection into three dimensions ${ }^{15-17}$ is needed, resulting in the 3D dose distribution delivered to a patient or a phantom.

In this work, we show the clinical results of routine pre-treatment verification and individual patient dosimetry. All procedures are based on electronic portal dosimetry (EPD) and carried out by radiation therapy technicians (RTTs). Potential sources of dose delivery errors in one point are obtained for all four error categories using one dose verification solution only and are discussed in detail. The work described in the current paper can be considered as the basis for a more elaborate 2D and 3D dose verification of other advanced treatment techniques such as IMRT, without changing the clinical procedure drastically.

\subsection{MATERIALS AND METHODS}

\subsubsection{Patients}

During 24 months, routine EPID-based pre-treatment verification, transit and in vivo dosimetry has been performed in our department; in this period over 37500 images were acquired and analyzed. An overview of the number of imaged patients and treatment plans is shown in Table 3.1. In total, 2511 patients and 3146 treatment plans were verified, mainly curative treatments of the pelvic region, breast, lung and head and neck region. Multiple treatment plans could be used per patient corresponding to different treatment phases and all plans were analyzed.

\subsubsection{Equipment}

All four SL15 linear accelerators (LINACS) in our department (Elekta, Crawley, United Kingdom) were used for this study. They are equipped with 6 and 10 MV photon beams, a motorized internal wedge and an intermediate-elbow videobased Theraview-NT EPID (Cablon Medical, Leusden, The Netherlands) consisting of a fluorescent screen, one mirror and a Peltier-cooled CCD camera (VDS Vosskühler $\mathrm{GmbH}$, Osnabrück, Germany) ${ }^{18}$. Grayscale EPID images were converted to portal dose images at a fixed distance of $150 \mathrm{~cm}$ from the accelerator focus by correcting for (1) 


\begin{tabular}{|c|c|c|c|c|c|c|}
\hline \multirow[b]{2}{*}{ Tumor site } & \multirow[b]{2}{*}{$\begin{array}{c}\text { \# of } \\
\text { patients }\end{array}$} & \multirow[b]{2}{*}{$\begin{array}{l}\text { \# of } \\
\text { plans }\end{array}$} & \multicolumn{2}{|c|}{$\begin{array}{l}\text { Pre-treatment } \\
\text { measurements }\end{array}$} & \multicolumn{2}{|c|}{$\begin{array}{l}\text { Patient dosimetry } \\
\text { measurements }\end{array}$} \\
\hline & & & $\begin{array}{c}\text { \# of } \\
\text { sessions }\end{array}$ & $\begin{array}{c}\text { \# of } \\
\text { beams }\end{array}$ & $\begin{array}{c}\text { \# of } \\
\text { sessions }\end{array}$ & $\begin{array}{c}\text { \# of } \\
\text { beams }\end{array}$ \\
\hline Pelvic regiona & 933 & 1,095 & 966 & 3,556 & 1,503 & 5,538 \\
\hline Breast & 725 & 1,001 & 872 & 2,230 & 873 & 2,348 \\
\hline Lung & 348 & 371 & 329 & 1,219 & 587 & 2,257 \\
\hline Head and neck & 242 & 371 & 343 & 1,207 & 414 & 1,696 \\
\hline Other & 192 & 236 & 202 & 583 & 263 & 811 \\
\hline Skull & 70 & 71 & 64 & 186 & 59 & 218 \\
\hline Vertebras & 1 & 1 & 2 & 10 & 2 & 11 \\
\hline Total & 2,511 & 3,146 & 2,778 & 8,991 & 3,701 & 12,879 \\
\hline
\end{tabular}

optical crosstalk in the EPID box, (2) non-linearity in the electronics, (3) differences in photon energy response and (4) non-uniformity in sensitivity of the fluorescent screen and CCD elements. This calibration has been extensively described for both a SRI-100 EPID and an iView EPID (Elekta, Crawley, United Kingdom), and is applied to all our Theraview-NT EPIDs and for all photon beam energies with the same accuracy as reported earlier ${ }^{7,8,19}$. Previous experience with the SRI-100 EPID and the iView EPID showed that portal dose values can be measured within 1-2\% compared to ionization chamber measurements ${ }^{7-8}$, under reference conditions. No electronic data transfer was present in our department during this study, thus treatment parameters from the TPS were manually entered into the accelerator console.

\subsubsection{Routine pre-treatment and patient dosimetry}

The dosimetric verification procedures were based on a single point dose value at the geometrical centre of a radiation field within a region-of-interest (ROI) of $0.5 \mathrm{~cm} \times 0.5$ $\mathrm{cm}$, taken from two-dimensional (2D) portal dose images.

For pre-treatment dosimetry, a measured central field dose was compared to a predicted dose value at the focus-EPID distance. The pre-treatment portal dose images were acquired during a dummy session with an open beam, before the actual treatment of a patient started and with the gantry positioned at $0^{\circ}$ to speed up the measurement procedure. For individual patient dosimetry, point dose comparisons were performed behind a patient at the position of the EPID (transit dosimetry) and at a depth of $5 \mathrm{~cm}$ inside the patient (in vivo dosimetry) using a back-projection of the measured central field dose. Both point dose comparisons have been described earlier by Pasma et al. ${ }^{13}$ but were only applied to 115 prostate cancer patients. Portal dose images were acquired during the first treatment sessions, with the patient on the 
treatment couch and with the gantry angles as prescribed by the treatment planning system. Routine clinical procedures like patient set-up correction by using off-line and on-line set-up protocols are not changed, and performed according to a treatment protocol. In this work, pre-treatment dose, transit dose and in vivo dose values are depicted by the symbols $D_{C}, D_{T}$ and $D_{5}$, respectively; the extensions "PLAN" and "EPID" indicate predicted and measured doses. Dose differences found during pre-treatment dosimetry had to be analyzed before the individual patient dosimetry could take place, thus eliminating data transfer and machine errors during the actual treatment of a patient.

RTTs performed both the measurements and the analysis of EPID images. An in-house developed software package (EPIDos) was used to perform the point dose predictions and back-projection, the conversion of EPID images into portal dose images and the point dose comparisons. Treatment parameters were electronically transferred from the TPS into EPIDos, but EPID images had to be read in manually. The throughput of the machine decreased by 10 min per pre-treatment verification measurement while no additional machine time was needed for individual patient dosimetry measurements. The analysis of the EPID images took less than $20 \mathrm{~s}$ per image if dose differences did not exceed prescribed action levels.

\subsubsection{Action levels}

For pre-treatment dosimetry, an action level of $5 \%$ was used for differences between predicted dose and measured dose values per beam, based on earlier experience ${ }^{8}$. If the action level was exceeded during pre-treatment verification, the treatment parameters from the treatment chart were checked again by the RTTs to verify that no errors were made during manual input, and the EPID measurements were repeated. If no valid explanation was found for the observations, or the action level was exceeded again after new measurements, a medical physicist was warned for further investigation; the analysis time per measurement then increased to 15-20 min.

For individual patient dosimetry, larger action levels were used that differed between tumor sites. These action levels were derived from initial clinical experience with the dosimetric verification procedures during the first three months of this study and are a trade-off between workload and ability to detect errors, aiming at a $90 \%$ rate of dose differences within the action levels. If an action level was exceeded, possible error sources of the dose differences were again identified and the impact of differences on the 3D dose delivery inside the patient was assessed qualitatively. The action level for the pelvic region was $7.5 \%$ for differences between predicted dose and measured dose per beam for both transit and in vivo dosimetry. For breast, lung and head and neck region, asymmetric action levels have been used which implies that systematic dose differences were neglected. This can be justified because error sources were recognized and the impact of the differences on the 3D dose delivery inside the patient was assessed. For instance, for breast treatments, action levels of $-17.5 \%$ and $+7.5 \%$ were used. The number of changes in treatment initiated by individual patient dosimetry was not registered during this work. 
Furthermore, the verification procedures had to be approved by a RTT or medical physicist before the treatment series continued.

\subsubsection{Dose calculation algorithms}

For pre-treatment verification, the central field dose value $D_{c, P L A N}$ was predicted by using an independent dose calculation algorithm which has been described earlier ${ }^{8,20}$. For transit dosimetry, the central field dose value was predicted behind a patient at the focus-EPID distance. This transit dose $D_{T, P L A N}$ was calculated by:

$D_{T, P L A N}=D_{c, \text { PLAN }} \cdot e^{-\mu(t) \cdot t} \cdot\left(1+S P R_{1}\right)$

with $t$ the radiological thickness of a patient or phantom along a ray line and $\mu$ the linear attenuation coefficient, which is a function of $t$ to correct for beam hardening. The scatter component originating in a patient was added to the central field dose as a scatter-to-primary ratio $\left(S P R_{1}\right)$, as described by Swindell et al. ${ }^{21}$.

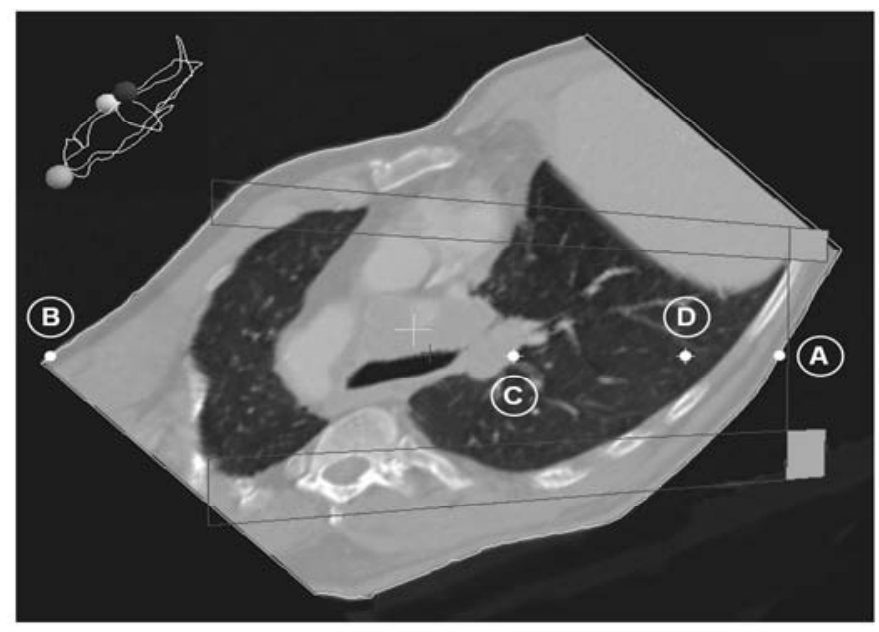

Figure 3.1: An oblique plane generated in the TPS for a lung treatment field going through the accelerator focus and the geometrical centre of the radiation field. A line through these two points is always oriented horizontally in the TPS by default and can be used to determine the beam entrance point (A), the beam exit point $(B)$ and the point located at a radiological depth of $5 \mathrm{~cm}$ (C). The latter point is determined by using a measurement tool in the TPS that expresses a distance between points both geometrically and radiologically. Note that the radiological depth $z_{5}$ of point $C$ can differ significantly from the geometrical depth of $5 \mathrm{~cm}$ of point $D$. The distance between points $A$ and $B$ is measured to determine the radiological thickness $t$; the distance between the accelerator focus and point $A$ is the focus-skin distance $S S D_{5}$. The dose at point $C$ is $D_{5, \text { PLAN. }}$

For the point dose comparison inside a patient, a radiological depth of $5 \mathrm{~cm}$ was used in this work. The in vivo dose $D_{5, P L A N}$ was determined in our TPS (XiO, Computerized 
Medical Systems, St. Louis, Missouri, USA) based on a convolution algorithm ${ }^{22}$. The measured in vivo dose at $5 \mathrm{~cm}$ radiological depth $D_{5, E P I D}$ was obtained by back-projecting the measured transit dose $D_{T, E P I D}$ :

$D_{5, \text { EPID }}=\frac{D_{T, E P I D}}{1+S P R_{1}} \cdot e^{\mu(t) t-\mu(5) \cdot 5} \cdot \frac{150^{2}}{\left(S S D_{5}+z_{5}\right)^{2}} \cdot\left(1+S P R_{2}\right)$

with $S S D_{5}$ the focus-skin distance along the ray line passing through accelerator focus and geometrical centre of a radiation field, $z_{5}$ the geometrical depth at radiological depth of $5 \mathrm{~cm}$ and $S P R_{2}$ the scatter-to-primary ratio due to patient scatter at this depth; $S P R_{2}$ is a function of the field area at $5 \mathrm{~cm}$ depth in water that is defined as $\left(c \cdot\left(S S D_{5}+z_{5}\right) / 100\right)^{2}$ with $c$ the equivalent square field size. Pasma et al. ${ }^{13}$ described an algorithm for $D_{5, E P I D}$ in prostate cancer patients where $S P R_{2}$ depends on $c$ and $S S D_{5}$ only; $z_{5}$ does not vary for this patient group $\left(z_{5}=5 \mathrm{~cm}\right)$.

The parameters $\mu, S P R_{1}$ and $S P R_{2}$ of Eqs. (3.1) and (3.2) were determined for homogeneous polystyrene slab phantoms with four thicknesses varying from 10 to $37.5 \mathrm{~cm}$ and six field sizes varying from $3 \mathrm{~cm} \times 3 \mathrm{~cm}$ to $24 \mathrm{~cm} \times 24 \mathrm{~cm}$. First, $\mu$ and $S P R_{1}$ were calculated by minimising the difference in $D_{T, P L A N}$ obtained by Eq. (3.1) and by measurements. Second, $S P R_{2}$ was determined by using predicted values of $D_{5, P L A N}$ from our TPS and calculated values of $D_{5, E P I D}$ from Eq. (3.2) with $D_{T, E P I D}$ from measurements. Dose differences between measured and predicted transit dose values $D_{T, P L A N}$ and in vivo dose values $D_{5, E P I D}$ were smaller than $0.5 \%$ (1 SD) for all measurements above. The parameters $t, S S D_{5}, z_{5}$ and $D_{5, P L A N}$ were determined using the TPS by RTTs using oblique planes going through the accelerator focus and the geometrical centre of a radiation field (Fig. 3.1).

\subsubsection{Analysis of dose differences}

Mean relative dose differences between predicted and measured central field doses were calculated as $\left(1-D_{E P I D} / D_{P L A N}\right) \cdot 100$ with corresponding standard deviations. A two-tailed paired Student's t-test was used to determine if dose differences were significantly different $(p<0.05)$, unless stated otherwise. Only those measurements approved by a RTT, i.e. $86-89 \%$ of all imaged treatment beams, were analyzed. Because measurements were mainly performed for treatments of the pelvic region, breast, lung and head and neck region, these were the only tumor sites which were analyzed separately. Dose differences larger than 50\% were excluded because these were not caused by actual dose delivery errors but by acquisition errors or errors made by the RTTs (e.g. erroneous determination of individual patient parameters $t, S S D_{5}, z_{5}$ and $\left.D_{5, P L A N}\right)$. In total, only 1 pre-treatment image and 59 treatment images were excluded.

Dose differences were determined per field segment, open and wedged segments separately, per treatment beam by summing dose values for the two segment types and per treatment session by adding the dose values for all beams of a treatment session. A summed in vivo dose value per treatment session does not actually describe the dose value in one specific point in the patient at $5 \mathrm{~cm}$ depth because different 


\begin{tabular}{|c|c|c|}
\hline Error category & $\begin{array}{l}\text { Dose } \\
\text { parameter }\end{array}$ & Error sources \\
\hline Acquisition errors & $D_{C} / D_{T} / D_{5}$ & $\begin{array}{l}\text { Errors during acquisition of EPID images (e.g. } \\
\text { malfunctioning of LINAC/EPID, wrong acquisition } \\
\text { parameters) }\end{array}$ \\
\hline User errors & $D_{C} / D_{T} / D_{S}$ & $\begin{array}{l}\text { Errors made by RTTs performing and analysing } \\
\text { measurements (e.g. erroneous determination of } \\
\text { individual patient parameters in TPS, images not } \\
\text { acquired or not saved) }\end{array}$ \\
\hline $\begin{array}{l}\text { Implementation } \\
\text { errors }\end{array}$ & $D_{C} / D_{T} / D_{5}$ & $\begin{array}{l}\text { Errors made during implementation of clinical } \\
\text { procedures (e.g. inaccuracies in measurements for } \\
\text { fitting dose calculation algorithms and dosimetric } \\
\text { calibration model of EPIDs, bugs in analysis software) }\end{array}$ \\
\hline $\begin{array}{l}\text { Procedure } \\
\text { limitations }\end{array}$ & $D_{C} / D_{T} / D_{5}$ & $\begin{array}{l}\text { Limitations in clinical procedures (e.g. limitations in } \\
\text { accuracy of dose calculation algorithms, limited EPID } \\
\text { FOV, geometrical centre of treatment field located } \\
\text { behind transmission block or close to field edges) }\end{array}$ \\
\hline $\begin{array}{l}\text { Transfer and } \\
\text { machine errors }\end{array}$ & $D_{C} / D_{T} / D_{5}$ & $\begin{array}{l}\text { Errors during data transfer from TPS to treatment } \\
\text { equipment and due to malfunctioning of treatment } \\
\text { machine (e.g. erroneous field sizes, number of monitor } \\
\text { units or collimatorangles are entered into the treatment } \\
\text { machine, gradual break-down of the monitor chamber } \\
\text { of the treatment machine) }\end{array}$ \\
\hline $\begin{array}{l}\text { Patient related } \\
\text { delivery errors }\end{array}$ & $D_{T} / D_{5}$ & $\begin{array}{l}\text { Errors caused by set-up errors or organ motion and due } \\
\text { to inaccuracies during the individualised treatment } \\
\text { planning process (e.g. occurrence of gas pockets in the } \\
\text { rectum, erroneous density correction in TPS) }\end{array}$ \\
\hline
\end{tabular}

gantry angles and dose point locations at the EPID are used. However, summed dose differences are more relevant, clinically, because a large dose difference for a segment with a small contribution to the total in vivo dose may be less important than a moderate dose difference in case of a very large contribution.

\subsubsection{Analysis of error sources}

An inventory was made of the error sources per treatment session if the dose difference for a minimum of one treatment beam exceeded the action level which means that the percentage of error sources for all treatment sessions is larger than the percentage of dose differences per beam exceeding the action level. Both approved and unapproved measurements were taken into account. All documented error sources were analyzed and subdivided into six categories: (1) acquisition errors, (2) user errors, (3) implementation errors, (4) procedure limitations, (5) transfer and machine errors and (6) patient related delivery errors (Table 3.2). The first four error categories can be seen as false positive dose delivery errors and should be absent if hardware and software are working flawlessly and no user errors are made. The latter 


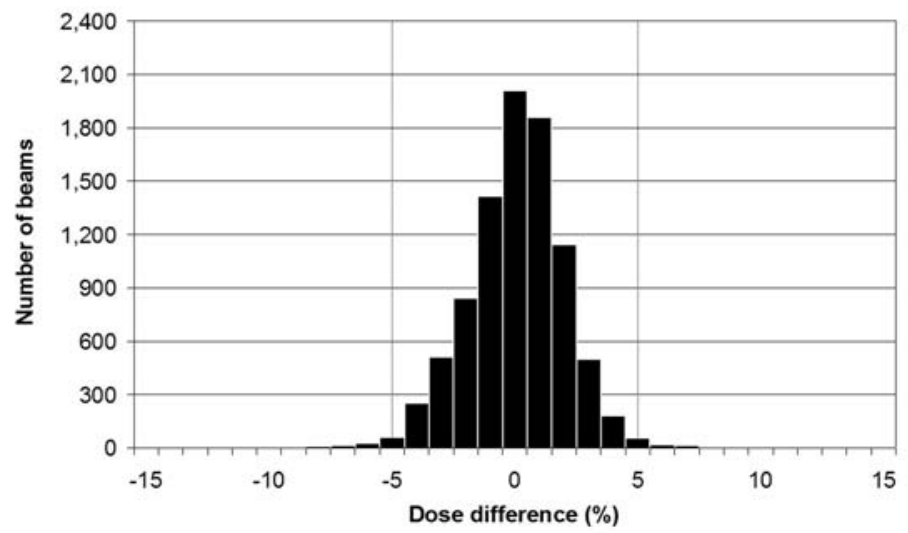

Figure 3.2: Relative dose differences between EPID dose measurements and dose calculations shown for 8991 treatment fields. The vertical lines indicate the 5\% action level used clinically. The mean dose difference is $0.1 \pm 2.4 \%$ (1SD).

two error categories represent real dose delivery errors which directly influence the accuracy of a radiotherapy treatment.

\subsection{RESULTS}

\subsubsection{Pre-treatment verification}

In Fig. 3.2, the relative pre-treatment dose differences per beam are shown with the clinically used action level of $5 \%$. About $97 \%$ of all data points lie within this action level. In Table 3.3, mean relative dose differences $D_{C}$ are shown for the different tumor sites, which vary per beam between $-1.2 \%$ for head and neck patients and $+1.2 \%$ for lung patients.

Errors were observed in $5 \%$ of all treatment sessions. These were errors causing dose differences at the EPID larger than the action level of $5 \%$ per beam. Limitations of the verification procedure caused false positive errors in $2 \%$ of all treatment sessions. One limitation was the verification of portal dose if the geometrical centre of a radiation field was shielded by transmission blocks or was positioned close to field edges. Transfer and machine errors were found for 12 patients (1\%) and these true positive errors were corrected before a patient was irradiated for the first time. For 4 patients, wrong field sizes were manually entered into the accelerator console and for 6 patients incorrect collimator angles were applied which led to an incorrect central field dose location. In one case, a shielding block was left out of the treatment description. Another error was a variation in machine output of about $4 \%$ for one linear accelerator. This change was due to the gradual break-down of the monitor chamber of the linear accelerator; the chamber was replaced immediately. 


\begin{tabular}{|c|c|c|c|c|c|}
\hline & & \multicolumn{4}{|c|}{$\begin{array}{l}\text { Mean relative dose difference and standard deviation } \\
\qquad(\%)\end{array}$} \\
\hline & & $\begin{array}{l}\text { Pelvic } \\
\text { region }\end{array}$ & Breast & Lung & $\begin{array}{l}\text { Head and } \\
\text { neck }\end{array}$ \\
\hline \multirow[t]{3}{*}{ Treatment beams } & $D_{C}$ & $0.7 \pm 1.7$ & $-0.8 \pm 2.3$ & $1.2 \pm 2.5$ & $-1.2 \pm 2.7$ \\
\hline & $D_{T}$ & $1.2 \pm 3.7$ & $-4.0 \pm 9.5$ & $-0.1 \pm 6.0$ & $-1.7 \pm 8.1$ \\
\hline & $D_{5}$ & $1.5 \pm 4.1$ & $-0.6 \pm 9.5$ & $-5.0 \pm 8.6$ & $2.4 \pm 8.0$ \\
\hline \multirow[t]{3}{*}{ Treatment sessions } & $D_{C}$ & $0.7 \pm 1.4$ & $-0.9 \pm 1.6$ & $1.2 \pm 1.3$ & $-0.8 \pm 1.7$ \\
\hline & $D_{T}$ & $1.1 \pm 2.4$ & $-4.4 \pm 7.1$ & $-0.2 \pm 4.2$ & $-2.1 \pm 5.7$ \\
\hline & $D_{5}$ & $1.4 \pm 3.0$ & $-1.1 \pm 6.6$ & $-5.4 \pm 5.9$ & $1.2 \pm 5.9$ \\
\hline
\end{tabular}

\subsubsection{Patient dosimetry of the pelvic region}

For the pelvic region, relative differences in transit dose $D_{T}$ and in vivo dose $D_{5}$ are shown in Fig. 3.3(a) and Table 3.3. Both dose comparisons give very similar results; about $97 \%$ and $95 \%$ of all transit and in vivo dose differences per beam are within the clinically used action levels, respectively. The action levels are also depicted in Fig. 3.3 for the two types of dose differences. Standard deviations are within $3 \%$ in case of summed dose differences per treatment session.

Errors were found and categorized in $24 \%$ of all treatment sessions, and are shown in Fig. 3.4. Most errors were user errors and patient related delivery errors. In case of the first category, many false positive errors were made during the determination of the individual patient parameters $t, S S D_{5}, z_{5}$ and $D_{5, P L A N}(4 \%)$. Also, images were not always analyzed and saved (1\%). In case of the second category, dose delivery errors were found due to differences in rectum filling (10\%) and patient set-up (3\%) between treatment planning and treatment delivery. For 35 patients, gas pockets were seen in the rectum resulting in transit dose and in vivo dose differences if the ray line through accelerator focus and geometrical centre of the radiation field passed through these pockets. For 9 patients, a set-up error was observed in combination with a hip prosthesis which sometimes led to dose differences of more than $10 \%$. For one patient, dose differences were caused by omission of a femoral head density correction during treatment planning.

\subsubsection{Patient dosimetry of the breast}

Large dose differences were observed when EPID patient dosimetry was applied during breast treatments (Fig. 3.3(b), Table 3.3). The mean transit dose difference for 

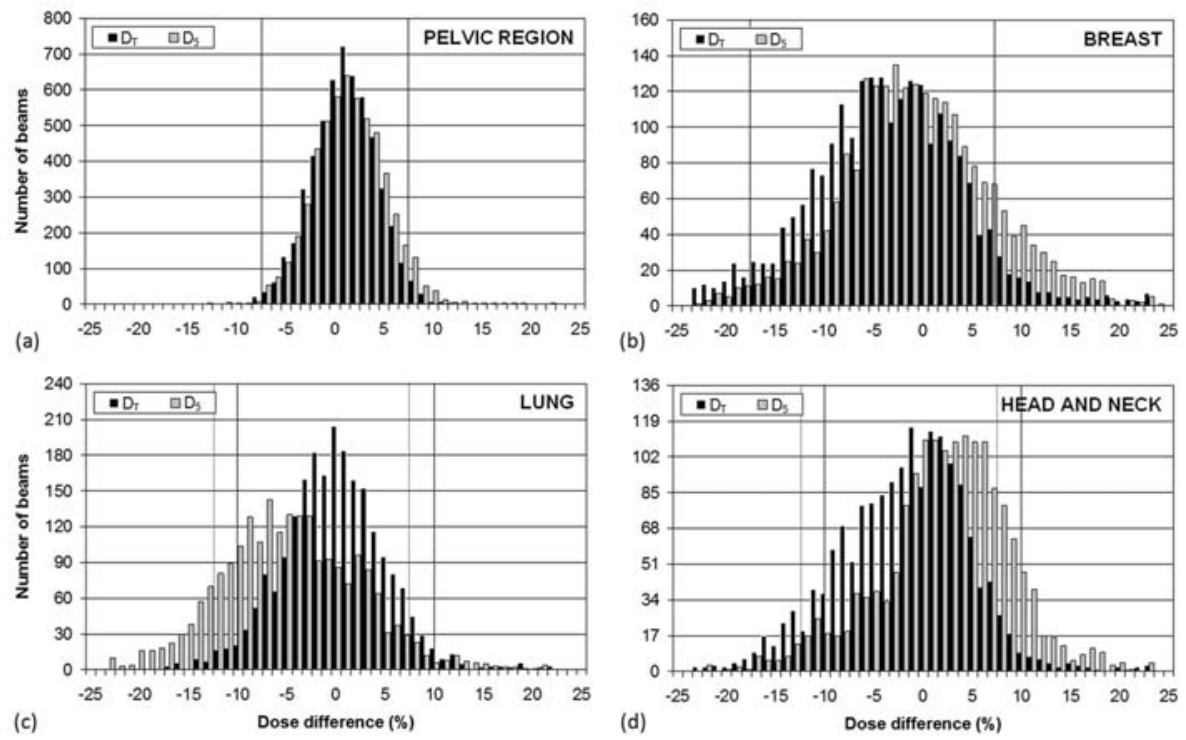

Fig. 3.3. Relative dose differences for (a) 5538 pelvic fields, (b) 2348 breast fields, (c) 2257 lung fields and (d) 1696 head and neck fields. Both differences in transit dose, $D_{T}$, and in vivo dose, $D_{5}$, are shown in combination with the corresponding action levels (solid and dotted vertical lines, respectively). The action levels for transit dosimetry and in vivo dosimetry are chosen independently based on initial clinical experience and can therefore be different, e.g. for the lung and head and neck patients.

individual beam verification is $-4.0 \%$ while the mean in vivo dose difference is $-0.6 \%$ $(p<0.001)$; the corresponding standard deviations are equal (both 9.5\%). In Fig. 3.3, the two Gaussian-shaped dose difference distributions are very similar but shifted with respect to each other. For the breast treatments, $90 \%$ and $85 \%$ of the transit dose and in vivo dose differences are within the clinically applied action levels of $-17.5 \%$ and $+7.5 \%$.

Error sources were identified for $49 \%$ of the treatment sessions, and are shown in Fig. 3.4. Most user errors were again made during determination of individual patient parameters and due to incomplete analysis of EPID images by RTTs. In case of procedure limitations, a part of the treatment couch construction blocked the dose point location in EPID images (4\%). Procedure limitations also concerned the use of simulator slices instead of CT slices during treatment planning causing incomplete structure sets (5\%). Also, for breast irradiations, the position of the treatment couch did not always allow EPID acquisition at $150 \mathrm{~cm}$ (3\%) making EPID dose verification at this distance impossible. Patient related delivery errors per beam were mostly caused by an irreproducible set-up of the patient (19\%). Comparison of EPID images with digitally reconstructed radiographs (DRRs) from the treatment planning system showed systematic set-up errors in the medial direction, sometimes up to $1.5 \mathrm{~cm}$. Finally, patient related delivery errors were also caused by the location of the 

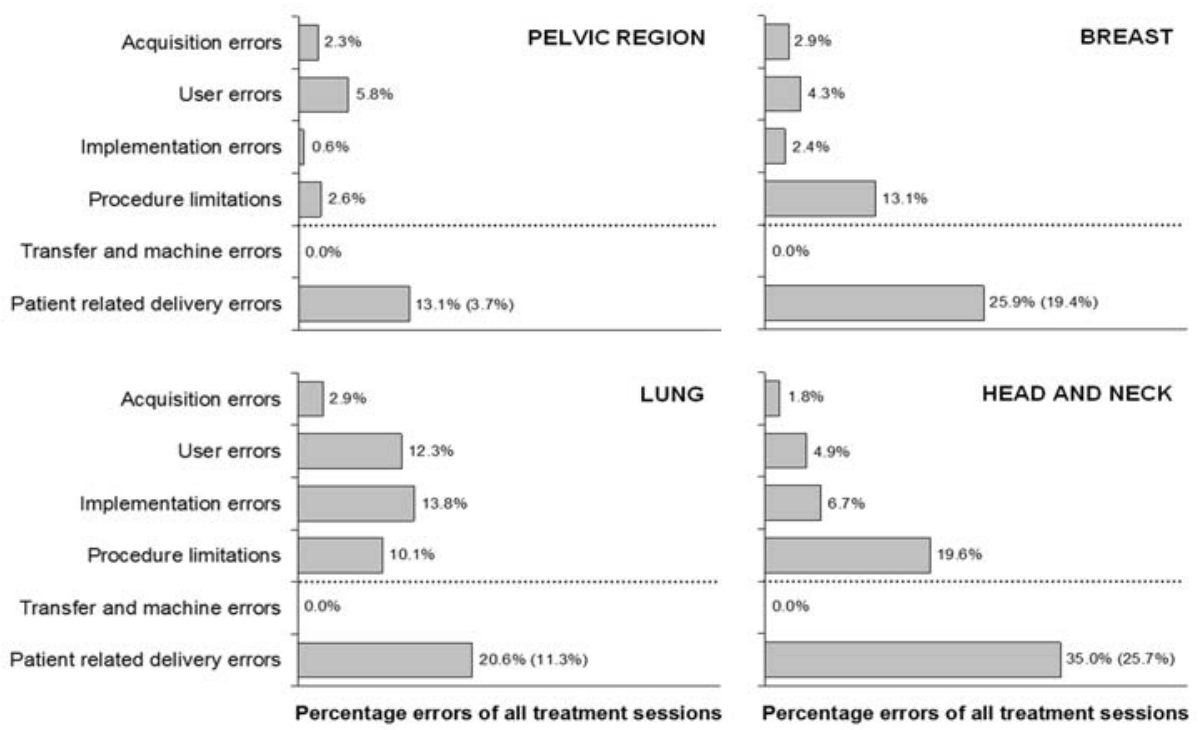

Figure 3.4: Distribution of error sources found for the pelvic region, breast, lung and head and neck patients using patient dosimetry measurements. Error sources are reported per treatment session and are expressed as a percentage of the total number of treatment sessions. A treatment session was considered erroneous if one or more beams exceeded the action level. In parentheses, the percentage of treatment sessions is shown where patient related delivery errors (true positive errors) resulted in a summed dose difference per treatment session larger than 5\%. The dotted horizontal line indicates a distinction between error sources leading to false positive and true positive dose delivery errors (above and below the line, respectively).

geometrical centre of the radiation field with respect to density interfaces in a patient (like the thorax hull or the patient's skin) in $7 \%$ of all treatment sessions.

\subsubsection{Patient dosimetry of the lung}

In case of patient dosimetry measurements for the lung, a significant difference was found between the results of transit dose and in vivo dose verification $(p<0.001)$. The measured dose inside the patient was systematically larger than the predicted in vivo dose $(p<0.001)$ while the mean transit dose difference was negligible $(p=0.13)$. Also, the corresponding standard deviations were smaller for the transit dose verification than for the in vivo dose verification (Fig. 3.3(c), Table 3.3). The same observation can be made by looking at the percentage of dose differences within the clinically used action levels: $94 \%$ for the transit dose and $83 \%$ for the in vivo dose.

In Fig. 3.4 the distribution of error sources for lung patient dosimetry with EPIDs is shown. Besides some of the user errors and procedure limitations already described earlier, the category with implementation errors was large (14\%). The most common error in this category was an error in the in-house developed analysis program, which affected the accuracy of the back-projection of $D_{T, E P I D}$ to obtain $D_{5, E P I D}$ (see 
Implementation errors). Real patient related delivery errors (21\%) were related to set-up errors of the patient and internal lung motion (15\%) and were also caused by the location of the geometrical centre of the radiation field with respect to density interfaces in and close to the lungs (6\%).

\subsubsection{Patient dosimetry of the head and neck region}

Patient dosimetry results for the head and neck region are shown in Fig. 3.3(d) and Table 3.3. As is the case for lung treatments, transit dose and in vivo dose verification gave different results but in head and neck, the mean in vivo dose difference was larger than the mean transit dose difference, $2.4 \%$ versus $-1.7 \%$, respectively $(p<0.001)$. Both standard deviations were almost equal. Of all transit and in vivo dose differences, $86 \%$ and $78 \%$ of the differences were smaller than the clinical action levels, respectively.

In Fig. 3.4, the error categorization is shown for head and neck treatment sessions. The implementation error that affected the accuracy of patient dose verification in case of lung treatment was also seen in head and neck treatments (5\%). Procedure limitations were found in case of $20 \%$ of all treatment sessions and were mainly caused by the treatment couch construction (9\%) or transmission blocks (6\%) shielding the dose point location in EPID images. Most user errors were made during determination of the individual patient parameters $t, S S D_{5}, z_{5}$ and $D_{5, P L A N}(3 \%)$. Besides these false positive errors, the occurrence of real patient related delivery errors per beam was also large for the head and neck region (35\% of all treatment sessions). For $21 \%$ of all treatment sessions, the point of measurement was located behind the trachea or behind tooth fillings, which made the dose verification very sensitive to set-up errors of a patient and organ motion. In case of irradiation of laryngeal cancer, the dose point was located close to the skin and sometimes even outside the patient (12\%). For 4 patients, a decrease in neck diameter was observed causing a dose difference larger than the action level.

\subsection{DISCUSSION}

All verification procedures described in this work are clinically applied on a routine basis by RTTs. Action levels and decision trees have been used to determine if dose differences had to be further investigated or could be accepted. These action levels have been determined at the start of our routine EPID dosimetry programme by looking at the results for small groups of patients. As a consequence, the number of dose differences within the clinically applied action levels and the percentage of positive errors differ for the different tumor sites as shown in Figs. 3.3 and 3.4. For $68 \%$ of all treatment sessions applied in the head and neck region, the dose difference for a minimum of one treatment beam exceeded the action level which was due to false positive errors (33\%) and true positive errors (35\%). This example shows that the false positive rate cannot be decreased independently from the true positive rate by simply using larger action levels. Therefore, the action levels have not been changed 
during this work. Using the measured dose differences and categorized errors per treatment sessions, action levels can be adapted to influence workload and error detection but, more importantly, verification procedures can also be optimized and all errors besides real dose delivery errors minimized. This means that smaller action levels can be used and smaller dose delivery errors can be detected and eliminated, improving the accuracy of absorbed dose delivery.

\subsubsection{Acquisition errors}

The percentage of acquisition errors that occurred for all 7450 treatment sessions that were analyzed for error categorization was $2 \%$. These errors were not only due to malfunctioning of the linear accelerator or EPID during the time of measurement but also due to wrong acquisition parameters. Although most acquisition parameters are defined by specific field characteristics which had to be transferred from the treatment planning system, the dosimetric acquisition protocol had to be chosen manually. If this step was forgotten, the standard acquisition protocol for set-up verification was applied using a variable integration time and number of frames. Using one acquisition protocol for both set-up verification and EPID dosimetry would decrease the number of acquisition errors.

\subsubsection{User errors}

The percentage of user errors for all analyzed treatment sessions was $4 \%$. Determination of individual patient parameters using oblique planes in the treatment planning system is a manual and less intuitive process explaining the large number of errors. These user errors can be eliminated by making this process automated. The parameters $t, S S D_{5}$ and $z_{5}$ can then be determined by segmentation of the 3D CT scan of a patient using the field properties from the treatment planning system, yielding the in vivo dose at $5 \mathrm{~cm}$ depth, $D_{5, P L A N}$, directly from the $3 \mathrm{D}$ dose distribution.

\subsubsection{Implementation errors}

The percentage of implementation errors for all analyzed treatment sessions was $3 \%$. For in vivo dosimetry of the lung and head and neck region, an implementation error in EPIDos was found. Instead of using $z_{5}$ determined in the treatment planning system, this depth was fixed to $5 \mathrm{~cm}$ in the EPIDos program code. Due to the lower density of lung, the average value of $z_{5}$ should be larger for lung verification (Fig. 3.1) which would decrease the reconstructed EPID patient dose (Eq. (3.2)). Assuming that transit dose differences and in vivo dose differences are equal, i.e. both $-0.1 \%$, the mean $z_{5}$ value was $7.2 \pm 3.4 \mathrm{~cm}$ (1 SD). For the head and neck patient group, this value would then be $3.0 \pm 3.6 \mathrm{~cm}$ ( $1 \mathrm{SD}$ ), which is smaller than $5 \mathrm{~cm}$ and can be explained by the higher density of bone in this region.

\subsubsection{Procedure limitations}

The percentage of errors due to procedure limitations for all analyzed treatment sessions was $6 \%$. Using EPIDs at a fixed distance of $150 \mathrm{~cm}$ from the accelerator focus 
limits the effective field-of-view (FOV) to $27 \mathrm{~cm} \times 27 \mathrm{~cm}$ isocentrically. The accuracy of the dose calibration model of the EPID decreases for treatment fields larger than the effective FOV because the optical crosstalk correction is fitted to a maximum field size of $24 \mathrm{~cm} \times 24 \mathrm{~cm}$. The effective FOV can be increased if the distance to the accelerator focus is decreased which is possible with the Theraview-NT EPIDs. However, a smaller focus-EPID distance decreases the number of treatment fields that can be imaged due to possible treatment couch collisions with the EPID. This will especially be the case for breast, lung and head and neck region. Also, the patient scatter contribution to the EPID increases significantly for a smaller patient-to-EPID distance, thus decreasing the accuracy of predicted and measured dose values for individual patient dosimetry ${ }^{23}$.

In case of breast treatments, not all treatment plans are CT-based. Using conventional simulator slices for treatment planning, only the body contour and lung contours are present. Missing structures like the clavicula in supraclavicular treatment fields can cause large transit and in vivo dose differences if the geometrical centre of a treatment field is positioned behind these structures. In this case, radiological thickness and radiological depths cannot be determined correctly. Also, contours derived at the simulator are less accurate than using CT scanning.

The dose prediction model and the back-projection model (Eqs. (3.1) and (3.2)) used in this work have a high accuracy if used at the geometrical centre of a treatment field but have also several limitations ${ }^{8}$. First, the prediction of $D_{C, P L A N}$ and $D_{T, P L A N}$ is less accurate if treatment fields are heavily shielded because the equivalent square field size $c$ is calculated only using the position of the collimating jaws. The decrease in collimator scatter due to shielding blocks is not taken into account, while the collimator exchange effect is neglected. The collimator exchange effect describes the difference in head scatter depending on the orientation of the collimator for asymmetric beams. Also, if the geometrical centre of a treatment field is located behind a transmission block, the predicted dose values $D_{c, \text { PLAN }}$ and hence $D_{\text {T,PLAN }}$ will be too high because the presence of the block is not taken into account. Using the transmission of the primary beam through a transmission block will increase the accuracy of dose verification for these cases. Another option is to perform the dose verification at a point close to the geometrical centre of a treatment field but not behind a transmission block. Furthermore, the prediction model of $D_{T, P L A N}$ and the back-projection model for $D_{5, E P I D}$ assumed a symmetrical distribution of inhomogeneities around the isocenter plane. In case of lung and head and neck region, this assumption is not always valid which causes a different patient scatter contribution to the EPID detector. However, the error in scatter-to-primary ratio $S P R_{1}$ (Eqs. (3.1) and (3.2)) will be very small ${ }^{23}$. Also, off-axis beam softening is neglected which results in less accurate results off-axis. Pasma et al. ${ }^{24}$ described a portal dose image prediction algorithm for open beams that incorporates off-axis beam softening but the input measurement set for this model is much more extensive. Because all point dose comparisons are performed at the geometrical centre of a radiation field in this work, the off-axis position and thus the inaccuracy of our dose calculation algorithms is limited. Finally, missing tissue is not taken into account which is particularly important for breast and laryngeal cancer which is treated with two opposing tangential fields. The scatter-to-primary ratios $S P R_{1}$ and $S P R_{2}$ will be both 
smaller than predicted in case of missing tissue and thus the model will overestimate the predicted transit $D_{T, P L A N}$; the back-projected in vivo dose $D_{5, E P I D}$ will also change depending on the change in ratio of $S P R_{2}$ and $S P R_{1}$.

\subsubsection{Transfer and machine errors}

The percentage of transfer and machine errors for all analyzed pre-treatment sessions was $1 \%$. Most transfer errors can be avoided if an electronic transfer would be present between TPS and treatment equipment. However, this means that all changes in treatment parameters should be updated in the TPS which was not always the case in our department, although this is the recommended procedure. Using the pre-treatment verification procedure, errors will be found and can trigger RTTs to look again at the actual treatment parameter values. For all in vivo dosimetry measurements, no transfer error was detected proving the effectiveness of the pre-treatment verification procedure.

\subsubsection{Patient related delivery errors}

For the pelvic region, dose differences were often found due to the presence of gas pockets in the rectum during treatment and not in the CT scan used for treatment planning. The actual in vivo dose will therefore vary but not daily in the same way because the presence of gas pockets is a random deviation. If the gas pockets are already present in the CT scan but not during treatment, differences can be systematic and replanning using a new CT scan or forcing densities is necessary. Due to changes in set-up, large dose differences were found in the case of a dose measurement behind a hip prosthesis. Correction of set-up errors by portal imaging set-up protocols decreased the dose differences and showed that the density correction in the treatment planning system is correct and hence the radiological thickness that was used for the hip prosthesis. In case of $4 \%$ of all treatment sessions (Fig. 3.4), patient related delivery errors per beam resulted in summed dose differences per treatment session larger than $5 \%$. These summed dose differences are more relevant clinically although summed dose differences do not describe the differences in prescribed fraction dose. However, they do indicate that the 3D in vivo dose distribution during treatment will differ locally from the distribution generated with the treatment planning system.

For breast treatments, systematic set-up errors are found in the medial direction decreasing the radiological thickness $t$. Both measured transit dose $D_{T, E P I D}$ and back-projected in vivo dose $D_{5, E P I D}$ will therefore increase equally (Eqs. (3.1) and (3.2)). Small set-up errors can cause large changes in diameter and dose differences due to the shape of a breast. Based on the systematic dose difference of $3.4 \%$ between the mean transit dose difference $(-4.0 \%)$ and the mean in vivo dose difference $(-0.6 \%)$ for breast treatments, the increase in back-projected in vivo dose $D_{5, E P I D}$ due to set-up is partially compensated by the decrease in $D_{5, E P I D}$ due to neglecting missing tissue in the dose back-projection. This means that the ratio of $S P R_{2}$ and $S P R_{1}$ is larger in case of missing tissue than under full-scatter conditions. In order to eliminate the large and systematic set-up errors in our department, treatment protocols are reviewed. Hector 
et $a{ }^{25}{ }^{25}$ reported a weighted average standard deviation in set-up of only $2.5 \mathrm{~mm}$ which is much smaller than the variations we found. Also, a set-up correction protocol for the breast will be implemented in our department, which should be applied before dose values are verified.

For breast, lung and head and neck region, patient dosimetry measurements could be made less sensitive to set-up errors and organ motion if the dose point is shifted away from the thorax hull, the patient's skin, density interfaces in and close to the lung and from trachea and tooth fillings. These positioning problems have also been observed during in vivo dosimetry using diodes ${ }^{26}$, e.g. for breast and head and neck region.

\subsubsection{Verification procedures}

The patient related delivery errors found in this work are very often related to set-up errors and organ motion. Also, changes in patient anatomy have been found, and incorrect density corrections during treatment planning have been detected. Both the transit dose and in vivo dose comparisons detect these errors while the pre-treatment dose comparison cannot. However, the pre-treatment verification is particularly useful in detecting dose delivery errors caused by treatment equipment and incorrect beam properties without uncertainties in portal dose due to the patient. Also, the pre-treatment procedure can be extended to verify other beam properties like presence of wedge, wedge direction, field size $^{8}$ and field shape. Care must be taken by interpreting the in vivo dose $D_{5, E P I D}$. This dose is calculated by using the individual patient parameters from the TPS in combination with the actual measured transit dose with all its uncertainties in patient anatomy due to set-up errors and organ motion. Still, the in vivo dose comparison might be very useful for detecting errors in the treatment planning software in absence of these patient uncertainties.

Measurements for individualized patient dosimetry were performed during the first treatment sessions of a treatment. At this time, set-up corrections are not yet applied in case of off-line set-up protocols and the patient's position and organ anatomy can still change during the rest of the treatment course. Hence, the outcome of the dosimetric verification procedures can change. To improve the clinical usefulness of our procedures, more measurements are needed spread over the entire treatment course, e.g. three times in the first week and then weekly, to be able to make a distinction between systematic and random dose delivery errors.

\subsubsection{Future developments}

In this work, the central field dose is checked pre-treatment which is useful for verification of the number of monitor units in case of conventional multi-segment treatment techniques and to detect machine output variations. We are currently in the process of adapting our method of pre-treatment verification for a more elaborate IMRT dose verification procedure by extending the central field point dose prediction to a $2 \mathrm{D}$ portal dose prediction. Besides the implementation of $2 \mathrm{D}$ pre-treatment verification with EPIDs for IMRT purposes, we are also in the process of changing the verification procedure and analysis software in order to perform 2D patient dosimetry. 
Therefore, we started to use the $2 \mathrm{D}$ portal dose prediction developed in our department ${ }^{23}$ profiting from the clinical experience obtained during the routine use of EPIDs for individualized patient dosimetry as described in this work. The 2D portal dose predictions will be compared to portal dose images obtained from amorphous silicon EPIDs that are very well suited for portal dosimetry applications ${ }^{6,27}$. Application of this type of EPID for point dose measurements will not change the conclusions of this work due to the high accuracy of the portal dose images from the video-based EPIDs. However, with 2D patient dosimetry, dose difference distributions can provide more insight into the type of error sources and location with respect to tumor and critical structures. Translation of transit dose differences to in vivo target dose differences will be done by using a 2D transit dose to $3 \mathrm{D}$ in vivo dose back-projection procedure ${ }^{17}$.

\subsection{CONCLUSIONS}

Pre-treatment verification and patient dosimetry procedures based on the use of EPIDs have been used clinically in our department for more than 24 months in which time about 2500 patient treatments were checked. Procedures could be fully applied by RTTs including acquisition, analysis and evaluation of about 37500 portal dose images. Treatment parameter transfer, machine output and dose delivery to a patient can be verified using EPIDs. Analysis of the data demonstrated false positive dose delivery errors due to the presence of user errors, implementation errors in the analysis software, procedure limitations but also true positive dose delivery errors. Using the measured dose differences and recognized error sources, verification procedures can be optimized and errors besides real dose delivery errors minimized. Transfer and machine errors possibly leading to dose delivery errors were found for $1 \%$ of all pre-treatment measurements. For $12 \%$ of all treatment sessions imaged during treatment, patient related error sources could be determined that probably affected the $3 \mathrm{D}$ in vivo dose distribution locally. Treatment imaging and set-up correction protocols are required to decrease dose differences particularly in case of breast treatments but also for lung, and head and neck irradiations.

\section{ACKNOWLEDGEMENTS}

The authors thank Esther Bloemen-van Gurp and Claudia Offermann for their help in supporting the individual patient dosimetry procedures with EPIDs clinically. 


\section{REFERENCES}

1 B.J. Mijnheer, J.J. Battermann, and A. Wambersie, "What degree of accuracy is required and can be achieved in photon and neutron therapy?," Radiother. Oncol. 8, 237-252 (1987).

2 J. van Dam, and G. Marinello, "Methods for in vivo dosimetry in external radiotherapy," Garant, Apeldoorn, The Netherlands, pp. 1-18 (1994).

3 S.C. Erridge, Y. Seppenwoolde, S.H. Muller, M.B. van Herk, K. De Jaeger, J.S.A. Belderbos, L.J. Boersma, and J.V. Lebesque, "Portal imaging to assess set-up errors, tumor motion and tumor shrinkage during conformal radiotherapy of non-small cell lung cancer," Radiother. Oncol. 66, 75-85 (2003).

4 C.W. Hurkmans, P. Remeijer, J.V. Lebesque, and B.J. Mijnheer, "Set-up verification using portal imaging; review of current clinical practice," Radiother. Oncol. 58, 105-120 (2001).

5 F. van den Heuvel, J. Fugazzi, E. Seppi, and J.D. Forman, "Clinical application of a repositioning scheme, using gold markers and electronic portal imaging," Radiother. Oncol. 79, 94-100 (2006).

6 L.N. McDermott, R.J.W. Louwe, J.J. Sonke, M.B. van Herk, and B.J. Mijnheer, "Dose-response and ghosting effects of an amorphous silicon electronic portal imaging device," Med. Phys. 31, 285-295 (2004).

7 K.L. Pasma, M. Kroonwijk, J.C.J. de Boer, A.G. Visser, and B.J.M. Heijmen, "Accurate portal dose measurement with a fluoroscopic electronic portal imaging device (EPID) for open and wedged beams and dynamic multileaf collimation," Phys. Med. Biol. 43, 2047-2060 (1998).

8 S.M.J.J.G. Nijsten, A.W.H. Minken, P. Lambin, and I.A.D. Bruinvis, "Verification of treatment parameter transfer by means of electronic portal dosimetry," Med. Phys. 31, 341-347 (2004).

9 J.J. Sonke, L.S. Ploeger, B. Brand, M.H.P. Smitsmans, and M.B. van Herk, "Leaf trajectory verification during dynamic intensity modulated radiotherapy using an amorphous silicon flat panel imager," Med. Phys. 31, 389-395 (2004).

10 A. Van Esch, T. Depuydt, and D.P. Huyskens, "The use of an aSi-based EPID for routine absolute dosimetric pre-treatment verification of dynamic IMRT fields," Radiother. Oncol. 71, 223-234 (2004).

11 M. van Zijtveld, M.L.P. Dirkx, H.C.J. de Boer, and B.J.M. Heijmen, "Dosimetric pre-treatment verification of IMRT using an EPID; clinical experience," Radiother. Oncol. 81, 168-175 (2006).

12 E. Bloemen-van Gurp, W. du Bois, P. Visser, I.A.D. Bruinvis, D. Jalink, J. Hermans, and P. Lambin, "Clinical dosimetry with MOSFET dosimeters to determine the dose along the field junction in a split beam technique," Radiother. Oncol. 67, 351-357 (2003).

13 K.L. Pasma, M. Kroonwijk, S. Quint, A.G. Visser, and B.J.M. Heijmen, "Transit dosimetry with an electronic portal imaging device (EPID) for 115 prostate cancer patients," Int. J. Radiat. Oncol., Biol., Phys. 45, 1297-1303 (1999).

14 M. van Zijtveld, M.L.P. Dirkx, H.C.J. de Boer, and B.J.M. Heijmen, "3D dose reconstruction for clinical evaluation of IMRT pretreatment verification with an EPID," Radiother. Oncol. 82, 201-207 (2007).

15 R. Boellaard, M. Essers, M.B. van Herk, and B.J. Mijnheer, "New method to obtain the midplane dose using portal in vivo dosimetry," Int. J. Radiat. Oncol., Biol., Phys. 41, 465-474 (1998).

16 V.N. Hansen, P.M. Evans, and W. Swindell, "The application of transit dosimetry to precision radiotherapy," Med. Phys. 23, 713-721 (1996). 
17 W.J.C. van Elmpt, S.M.J.J.G. Nijsten, R.F.H. Schiffeleers, A.L.A.J. Dekker, B.J. Mijnheer, P. Lambin, and A.W.H. Minken, "A Monte Carlo based three-dimensional dose reconstruction method derived from portal dose images," Med. Phys. 33, 2426-2434 (2006).

18 E.M. Franken, J.C.J. de Boer, J.C. Barnhoorn, and B.J.M. Heijmen, "Characteristics relevant to portal dosimetry of a cooled CCD camera-based EPID," Med. Phys. 31, 2549-2551 (2004).

19 B.J.M. Heijmen, K.L. Pasma, M. Kroonwijk, V.G.M. Althof, J.C.J. de Boer, A.G. Visser, and H. Huizenga, "Portal dose measurement in radiotherapy using an electronic portal imaging device (EPID)," Phys. Med. Biol. 40, 1943-1955 (1995).

20 B. Smulders, I.A.D. Bruinvis, and B.J. Mijnheer, "Monitor unit calculations for wedged asymmetric photon beams," Phys. Med. Biol. 47, 2013-2030 (2002).

21 W. Swindell, and P.M. Evans, "Scattered radiation in portal images: a Monte Carlo simulation and a simple physical model," Med. Phys. 23, 63-73 (1996).

22 M. Miften, M. Wiesmeyer, S. Monthofer, and K. Krippner, "Implementation of FFT convolution and multigrid superposition models in the FOCUS RTP system," Phys. Med. Biol. 45, 817-833 (2000).

23 W.J.C. van Elmpt, S.M.J.J.G. Nijsten, B.J. Mijnheer, and A.W.H. Minken, "Experimental verification of a portal dose prediction model," Med. Phys. 32, 2805-2818 (2005).

24 K.L. Pasma, B.J.M. Heijmen, M. Kroonwijk, and A.G. Visser, "Portal dose image (PDI) prediction for dosimetric treatment verification in radiotherapy. I. An algorithm for open beams," Med. Phys. 25, 830-840 (1998).

25 C.L. Hector, S. Webb, and P.M. Evans, "The dosimetric consequences of inter-fractional patient movement on conventional and intensity-modulated breast radiotherapy treatments," Radiother. Oncol. 54, 57-64 (2000).

26 C. Fiorino, D. Corletto, P. Mangili, S. Broggi, A. Bonini, G.M. Cattaneo, R. Parisi, A. Rosso, P. Signorotto, E. Villa, and R. Calandrino, "Quality assurance by systematic in vivo dosimetry: results on a large cohort of patients," Radiother. Oncol. 56, 85-95 (2000).

27 J. Chen, C.F. Chuang, O. Morin, M. Aubin, and J. Pouliot, "Calibration of an amorphous-silicon flat panel portal imager for exit-beam dosimetry," Med. Phys. 33, 584-594 (2006). 


\title{
INTRODUCTION
}

\author{
POINT DOSE VERIFICATION \\ METHODS AND APPLICATION
}

TWO-DIMENSIONAL TRANSIT DOSIMETRY METHODS AND APPLICATION

DISCUSSION 



\section{CHAPTER}

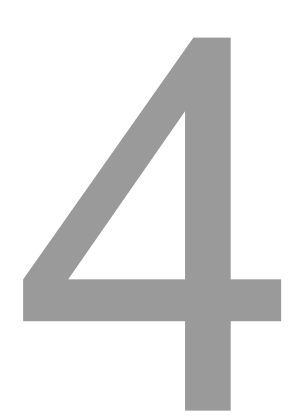

Experimental verification of a portal dose prediction model

Med. Phys. 32(9), 2805-2818 (2005)

W.J.C. van Elmpt* S.M.J.J.G. Nijsten* B.J. Mijnheer A.W.H. Minken * these authors equally contributed to this study 



\section{ABSTRACT}

Electronic portal imaging devices (EPIDs) can be used to measure a two-dimensional (2D) dose distribution behind a patient, thus allowing dosimetric treatment verification. For this purpose we experimentally assessed the accuracy of a 2D portal dose prediction model based on pencil beam scatter kernels. A straightforward derivation of these pencil beam scatter kernels for portal dose prediction models is presented based on phantom measurements. The model is able to predict the 2D portal dose image (PDI) behind a patient, based on a PDI without the patient in the beam in combination with the radiological thickness of the patient. To assess the accuracy of portal dose and radiological thickness values obtained with our model, various types of homogeneous as well as inhomogeneous phantoms were irradiated with a $6 \mathrm{MV}$ photon beam. With our model we are able to predict a PDI with an accuracy better than $2 \%$ (mean difference) if the radiological thickness of the object in the beam is symmetrically situated around the isocenter. For other situations deviations up to $3 \%$ are observed for a homogeneous phantom with a radiological thickness of $17 \mathrm{~cm}$ and a $9 \mathrm{~cm}$ shift of the midplane-to-detector distance. The model can extract the radiological thickness within $7 \mathrm{~mm}$ (maximum difference) of the actual radiological thickness if the object is symmetrically distributed around the isocenter plane. This difference in radiological thickness is related to a primary portal dose difference of $3 \%$. It can be concluded that our model can be used as an easy and accurate tool for the $2 \mathrm{D}$ verification of patient treatments by comparing predicted and measured PDIs. The model is also able to extract the primary portal dose with a high accuracy, which can be used as the input for a 3D dose reconstruction method based on back-projection. 


\subsection{INTRODUCTION}

Electronic Portal Imaging Devices (EPIDs) acquire a two-dimensional (2D) photon transmission distribution behind a patient during external beam radiotherapy. ${ }^{1}$ EPIDs can be used for two main purposes. First, as imaging devices: e.g., a portal image can be compared with a digitally reconstructed radiograph derived from a planning CT scan to detect errors in patient set-up. Furthermore portal images can be used for verifying leaf position for instance as applied during intensity-modulated radiotherapy treatments. In addition EPIDs can be used as dosimetry devices. After being calibrated EPIDs are used for portal dosimetry purposes. ${ }^{2-4}$ This last application is currently not used at its full potential but offers a lot of extra possibilities for the quality control of a treatment with external photon beams.

Quality control procedures of a patient treatment can be divided into two main parts; first quality control of the technical aspects of the linear accelerator itself, and second verification of the actual patient treatment. A technical quality control program concerns, amongst others, verification of: The output of the linear accelerator, the parameter transfer between treatment planning system and linac, the collimator settings and the direction of wedges. ${ }^{5}$ Quality control of a patient treatment includes verification of both the patient set-up and of the delivery of the correct dose. With in vivo dosimetry, the actual dose delivered to the patient during treatment is checked. Several detector types such as diodes, thermoluminescence dosimeters (TLDs) or metal oxide field effect transistors (MOSFETs) can be used for this purpose. ${ }^{6-8}$ Portal dosimetry can also be used for in vivo dosimetry purposes because the portal dose can be related to the patient dose. This has lead to various possibilities for in vivo dosimetry applying EPIDs.

Several portal dose prediction models have been developed. ${ }^{9-12}$ These models calculate the dose at the position of the EPID, which can then be compared with the measured portal dose during treatment. If the predicted and measured portal dose distributions are equal, then the actual dose delivered to the patient is assumed to be the same as the planned dose. However, if there are deviations between the two dose distributions it may be difficult to interpret the differences in terms of patient dose. Therefore exit-plane reconstructions ${ }^{13,14}$ and midplane reconstruction ${ }^{15-17}$ methods have been developed for the verification of the planned dose inside a patient. The reconstructed dose can be compared with the planned dose obtained from the treatment planning system in these planes.

However, portal dose images (PDIs) are not only useful to verify whether the planned portal dose is the same as the measured portal dose, but can also be used to perform a full three-dimensional (3D) reconstruction of the actual dose delivered to the patient. For such a 3D dose reconstruction so-called back-projection methods have been developed. ${ }^{16-21}$ These back-projection methods relate the primary portal dose to the dose actually delivered to the patient. For these methods it is necessary to separate the primary dose from the patient scattered dose at the position of the EPID. ${ }^{22-25}$ The scattered dose comes from all irradiated parts of the patient, while the primary portal dose is only related to the radiological thickness of the path traversed by the photons 
in the patient. This primary portal dose can then be back-projected through a model of the patient during treatment. The total dose inside the patient can be calculated from the primary dose by adding the scattered patient dose component. To make this dose reconstruction completely independent of the planning CT data, a CT scan of the patient in treatment position has to be made. Recent developments with megavoltage ${ }^{26}$ or kilovoltage ${ }^{27}$ cone-beam CT scans show this is a feasible method for acquiring such a CT scan either during or just before the actual treatment.

Currently in our department a portal dose prediction model is used clinically that calculates the portal dose at the center of the field. This calculated point dose is compared with a single point dose obtained from a measured two-dimensional, 2D, PDI. The ultimate goal of our in vivo dosimetry program is, however, not to verify the dose at a point or plane behind the patient, but to check the dose actually delivered to the patient.

The aim of this study was to extend the point dose prediction model currently used in our department to a 2D portal dose prediction model. Portal dose prediction methods, based on pencil beam scatter kernels can predict the portal dose with high accuracy. ${ }^{10,12}$ The model presented is based solely on the radiological thickness of the patient along a ray line and a portal dose image taken prior to treatment without the patient in the beam. To use this model in the future for 3D dose reconstruction, the model has to be able to extract the primary portal dose from a measured PDI during treatment. The accuracy of the 2D portal dose prediction and the extraction of the primary portal dose of the model were tested in an experimental study including homogeneous as well as inhomogeneous phantoms. In order to illustrate the application of our 2D portal dose prediction model, a clinical example is also given.

\subsection{THEORETICAL MODEL}

A model is used that describes the relation between three sets of data: Two PDIs, one with and a second without an object in the beam, and the radiological thickness of the path traversed by photons in the object. With object in this paper either a patient or a phantom is meant. If two of these quantities are known, the third one can be calculated. In this study we analyzed the accuracy of two options of the model. First, we studied the prediction of the portal dose with an object in the beam based on the radiological thickness of the object and a PDI taken without the object in the beam. Second, the extraction of the radiological thickness from a PDI with and without object in the beam was evaluated.

We adapted the original portal dose prediction model developed by Pasma et al. ${ }^{10}$ at three points. First, the portal dose prediction model has been extended to be able to extract also the radiological thickness of the patient from the two PDIs. Radiological thickness is related to the primary portal dose by the effective attenuation coefficient along the ray-lines. Pasma et al. $^{28}$ used their model in a similar way to verify the radiological thickness of compensators. 
Second, the pencil beam scatter kernels were fitted to a pre-defined kernel function. The derivation of these pencil beam scatter kernels is not straightforward. The original model used an iterative calculation of the pencil beam scatter kernels from phantom measurements, but these kernels showed a non-physical behavior near the beam axis which averaged out in the calculation of the scattered portal dose. Monte Carlo simulations of the scatter kernels ${ }^{24,29}$ are also presented in the literature but this approach requires complex calculations, while in addition such a Monte Carlo method is not available in our department. Analytically derived scatter kernels ${ }^{25,30}$ have also been presented but these kernels are only an estimate of the photon fluence or do not represent the detector response. We developed a simple method that describes the pencil beam scatter kernel with a pre-defined kernel function. The coefficients of this function are fitted to the measured phantom scattered component of the transmission, discussed in Sec. 4.2.2. We analyzed three of these predefined functions based on either physical properties or empirical assumptions. Our method does not need complex Monte Carlo calculations and requires only a limited set of transmission measurements. Predictions are made for a fixed focus-detector distance of $150 \mathrm{~cm}$, being the focus-detector distance of our EPID. Scatter kernels were calculated at this distance for a set of homogeneous phantoms placed symmetrically around the isocenter located at $100 \mathrm{~cm}$ to the focus, resulting in a phantom midplane-to-detector distance of $50 \mathrm{~cm}$.

Third, the prediction of a portal dose image is based on the assumption that the radiological midplane coincides with the isocenter plane. This is a simplified version of the equivalent homogeneous phantom (EHP) concept described by Pasma et al. ${ }^{10}$ This concept replaces an inhomogeneous object by a homogeneous object having the same radiological thickness along an incident ray line from focus to detector. Its thickness is repositioned in such a way that it is distributed symmetrically around the center-of-mass of the particular ray line. The EHP consists of two arrays: An array of radiological thicknesses and an array of distances from the center-of-mass to the portal dose plane. Their approach was modified in such a way that the model is only depending on one parameter: The radiological thickness of the object in the beam. This simplified version was chosen to make the extraction of the radiological thickness totally independent of the object in the beam.

The extended model can operate in two ways, yielding different types of information. First, it can predict a PDI with an object in the beam, based on a PDI without the object and its radiological thickness. Second, it allows the extraction of the radiological thickness based on a PDI with and without the object in the beam.

\subsubsection{Prediction of 2D portal dose images}

Briefly, the model predicts the primary portal dose $P(x, y)$ and the phantom scattered portal dose $S(x, y)$ at a location $(x, y)$ at the EPID of an object with radiological thickness $t_{(x, y)}$ based on a PDI without the object in the beam $O(x, y)$ :

$$
P(x, y)=O(x, y) \exp \left(-\mu\left(r_{(x, y)}, t_{(x, y)}\right) \cdot t_{(x, y)}\right)
$$




$$
S(x, y)=\iint_{x^{\prime}, y^{\prime} \in \text { Field }} O\left(x^{\prime}, y^{\prime}\right) K\left(r_{\left(x-x^{\prime}, y-y^{\prime}\right)}, t_{\left(x^{\prime}, y^{\prime}\right)}, d\right) \cdot d x^{\prime} d y^{\prime}
$$

in which $\mu\left(r_{(x, y)}, t_{(x, y)}\right)$ is the effective attenuation coefficient for a phantom with thickness $t_{(x, y)}$ at position $r_{(x, y)}=\mathrm{V}\left(x^{2}+y^{2}\right)$ and $K(r, t, d)$ a scatter kernel for phantom thickness $t$ and midplane-to-detector distance $d$. The phantom scattered dose component is obtained by a superposition of the total energy released per mass (TERMA) in the EPID with the scatter kernel $K$. TERMA is equal to the product of the energy fluence and mass-attenuation coefficient at the position of the EPID, and is proportional to $O(x, y)$. The effective depth of the points $(x, y)$ in the EPID is $2.5 \mathrm{~cm}$ in water equivalent material, which is also the depth in the large water phantom where the verification measurements were performed. $P, O$, and $S$ are, therefore, defined at this depth. Note that the prediction of the primary dose is independent of the midplane-to-detector distance of the phantom and is only related to the off-axis location and phantom thickness.

Only two model parameters are required. First, a set of radial symmetric scatter kernels for various phantom thicknesses $t$ placed with a midplane-to-detector distance $d$. Thicknesses not included in the set of scatter kernels are derived using a linear interpolation. Second, the model includes an effective attenuation coefficient, which depends on both off-axis distance (to include beam-softening) and phantom thickness (to include beam-hardening). The summation of the primary and scattered portal dose distribution yields the total portal dose distribution $I(x, y)$.

\subsubsection{Derivation of the model parameters}

\subsubsection{Effective attenuation coefficient}

The total transmission $T(x, y)$ of a photon beam behind a homogeneous phantom is defined as the sum of the primary dose $P(x, y)$ and the phantom scattered dose $S(x, y)$ divided by the dose $O(x, y)$ without the phantom in place. The transmission consists of a primary component $T^{P}(x, y)=P(x, y) / O(x, y)$ and a component $T^{S}(x, y)=S(x, y) / O(x, y)$ arising from phantom scatter:

$$
T(x, y)=\frac{P(x, y)+S(x, y)}{O(x, y)}=T^{p}(x, y)+T^{S}(x, y)
$$

The primary component of the transmission is only related to the radiological thickness $t$ of the phantom and the energy spectrum of the beam, both effects are described by the effective attenuation coefficient. This primary component can be estimated by extrapolating the total transmission to a field size $A$ of $0 \mathrm{~cm} \times 0 \mathrm{~cm},{ }^{10}$

$$
T^{p}(x, y)=\lim _{A \downarrow 0} T(x, y)=\exp \left(-\mu\left(r_{(x, y)}, t\right) \cdot t\right)
$$




\subsubsection{Pencil beam scatter kernels}

The phantom scattered component of the transmission depends on a number of physical parameters such as phantom thickness, field size and midplane-to-detector distance. This phantom scattered component can be estimated by subtracting the primary component from the total transmission. The on-axis phantom scattered part behind a homogeneous object with thickness $t$ can be calculated by a superposition of the incident field with pencil beam scatter kernels: $:^{10,12,30}$

$$
T^{s}(x=0, y=0)=\iint_{(x, y) \text { Field }} \frac{O(x, y)}{O(x=0, y=0)} \cdot K\left(\sqrt{x^{2}+y^{2}}, t, d\right) d x d y
$$

We investigated three radial symmetric functions $K(r, t, d)$ to describe the pencil beam scatter kernel. (1) A Gaussian shape $K_{\text {Gauss }}$ [Eq. (4.6)] described by two parameters $c_{1}^{r e f}(t)$ and $c_{2}^{r e f}(t) ;(2)$ a function based on an isotropic point source $K_{\text {Isotropic }}$ [Eq. (4.7)] described by two parameters indicating the strength $c_{1}^{r e f}(t)$ and the distance $c_{2}^{\text {ref }}(t)$ of the (virtual) point source above the detector plane; (3) a function based on single Compton scattered photons described by the Klein-Nishina cross-section $K_{K N}$ [Eq. (4.8)] with variable strength $c_{1}{ }^{\text {ref }}(t)$.

$$
\begin{aligned}
& K_{\text {Gauss }}(r, t, d)=\frac{c_{1}^{\text {ref }}(t)}{s} \exp \left(-\left(\frac{r}{s \cdot c_{2}^{\text {ref }}(t)}\right)^{2}\right) \\
& K_{\text {Isotropic }}(r, t, d)=\frac{s^{2} \cdot c_{1}{ }^{\text {ref }}(t) \cdot c_{2}^{\text {ref }}(t)}{\left(r^{2}+\left(s \cdot c_{2}{ }^{\text {ref }}(t)\right)^{2}\right)^{3 / 2}} \\
& K_{K N}(r, t, d)=\frac{c_{1}(t)}{2}\left(\frac{1}{1+\alpha(1-\cos \theta)}\right)^{2}\left(1+\cos ^{2} \theta+\frac{\alpha^{2}(1-\cos \theta)^{2}}{1+\alpha(1-\cos \theta)}\right) \frac{\cos ^{3} \theta}{d^{2}}
\end{aligned}
$$

with $s=\left(d / d^{\text {ref }}\right)^{2}$ a scaling factor taking into account the midplane-to-detector distance $d, \cos \theta=d / \mathrm{v}\left(r^{2}+d^{2}\right)$ describing the angle $\theta$ of the scattered photons, $\alpha=E / m_{0} c^{2}$ is the average photon energy $E$ expressed in units $m_{0} c^{2}=0.511 \mathrm{MeV}$ and $c_{1}{ }^{\text {ref }}(t), c_{2}{ }^{\text {ref }}(t)$ are fitting coefficients of the scatter kernel for phantom thickness $t$ placed at midplane-to-detector distance $d^{\text {ref }}$. Pencil beam scatter kernels for other midplane-to-detector distances $d$ than the fitting geometry $d^{\text {ref }}$ are taken into account by the scaling factor $s$ which is based on divergence of the scattered photons created in the midplane of the phantom.

\subsubsection{Extraction of radiological thickness}

The model can also be used to extract the radiological thickness of an object from two PDIs, with and without an object in the beam. An iterative technique can be used $d^{24,28}$ to extract the radiological thickness of the object. Only a limited number of iteration steps are needed for the solution to converge. ${ }^{31,32}$ All iterations in this study were performed with three iteration steps. The iterative loop for the extraction of the radiological thickness is shown in Fig. 4.1. 


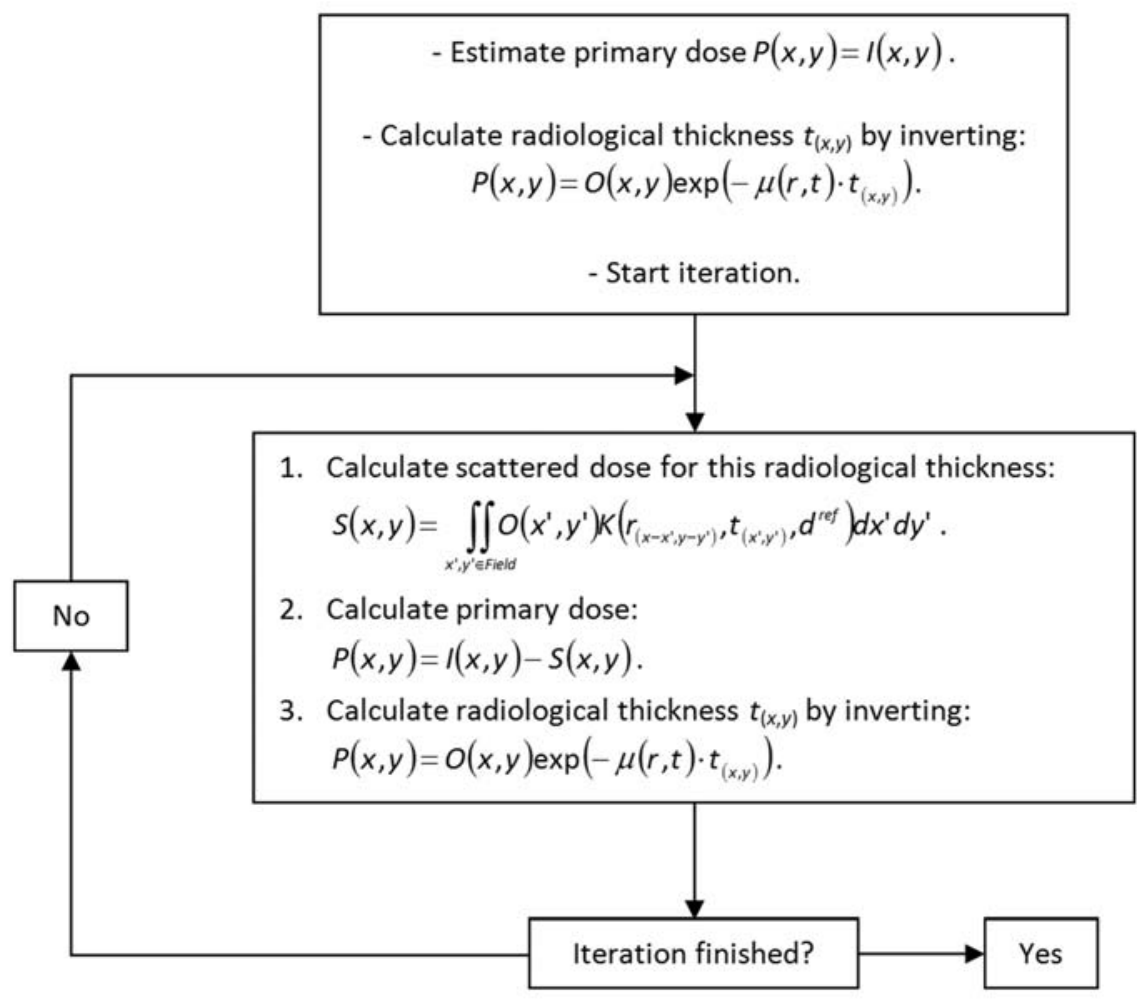

Figure 4.1: Flow chart showing the iterative process for the extraction of the radiological thickness from EPID measurements. All iterations in this study were performed with three steps.

\subsection{MATERIALS AND METHODS}

\subsubsection{Detectors and measurement set-up}

All measurements were performed with a $6 \mathrm{MV}$ photon beam from a SL15 linear accelerator (Elekta, Crawley, United Kingdom) under full scatter conditions in a water phantom (Blue Phantom, Scanditronix Wellhöfer, Schwarzenbruck, Germany) with two types of detectors. First, an ionization chamber (CC13, Scanditronix Wellhöfer, Schwarzenbruck, Germany), read out by a UNIDOS E electrometer (PTW, Freiburg, Germany), was used for absolute dosimetry. Second, a linear detector array (CA24, Scanditronix Wellhöfer, Schwarzenbruck, Germany) with 23 ionization chambers spaced at an equal distance of $2 \mathrm{~cm}$ and read out by a multichannel electrometer (MD240, Scanditronix Wellhöfer, Schwarzenbruck, Germany) was used in the dose rate mode with an additional ionization chamber (CC13) positioned inside the field to correct for a possible drop in dose rate of the linac. The water surface was positioned at $147.5 \mathrm{~cm}$ from the source and the effective centers of the detectors are positioned 
$2.5 \mathrm{~cm}$ below the water surface, resulting in a source-to-detector distance (SDD) of 150 $\mathrm{cm}$. These conditions are equal to the calibration conditions of the EPID.

With the linear detector array it is possible to scan a 2D dose profile, whereas an additional absolute point dose measurement is used to convert this relative dose distribution to an absolute dose distribution. This 2D measured portal dose distribution has measurement points every $2 \mathrm{~cm}$ both in $x$ and $y$ direction which is then linearly interpolated to a grid size equal to the dose grid size of the EPID.

For the measurement of a PDI in clinical situations we used a CCD-based electronic portal imaging device EPID (Theraview NT, Cablon Medical, Leusden, The Netherlands), which was calibrated to measure dose under full scatter conditions. ${ }^{2-5}$ The grid size of the EPID after dosimetric calibration is $128 \times 128$ pixels with an effective detector area of $40 \mathrm{~cm} \times 40 \mathrm{~cm}$, resulting in an effective grid spacing of $0.3 \mathrm{~cm}$. The SDD of the EPID was $150.0 \mathrm{~cm}$.

\subsubsection{Derivation of the model parameters}

\subsubsection{Derivation of the effective attenuation coefficient}

Absolute point dose measurements on the central beam axis and at off-axis points $r=3$, 6 , and $9 \mathrm{~cm}$, with distances expressed at the isocenter plane, were performed using symmetric fields having widths of $3-5 \mathrm{~cm}$, situated symmetrically around the measurement point. The primary component of the transmission and the effective attenuation coefficient were determined by a linear extrapolation of the measured transmission for field sizes of $5 \mathrm{~cm} \times 5 \mathrm{~cm}, 4 \mathrm{~cm} \times 4 \mathrm{~cm}$, and $3 \mathrm{~cm} \times 3 \mathrm{~cm}$ to a field area of $0 \mathrm{~cm} \times 0 \mathrm{~cm}^{10}$

\subsubsection{Derivation of the pencil beam scatter kernels}

Absolute point dose measurements on the central beam axis were performed using symmetric field widths of $3,6,10,15,21$, and $24 \mathrm{~cm}$. For each measurement 200 monitor units were given. Also a PDI $O(x, y)$ of the largest field was measured.

Phantoms were made of stacked layers of polystyrene (PS) with a density of $1.01 \mathrm{~g} / \mathrm{cm}^{3}$ having dimensions of $29 \mathrm{~cm} \times 29 \mathrm{~cm}$ and thicknesses of 4.3, 8.6, 12.9, 17.1, and 21.4 $\mathrm{cm}$. For the fitting of the kernel functions the phantoms were placed symmetrically around the isocenter plane located at $100 \mathrm{~cm}$ from the source, i.e., a fixed midplane-to-detector distance $d=d^{\text {ref }}=50 \mathrm{~cm}$. The phantom scattered component of the transmission was calculated by subtracting the primary component from the total transmission.

The parameters $c_{1}{ }^{r e f}(t)$ and $c_{2}{ }^{r e f}(t)$ of the kernel functions in Eqs. (4.6)-(4.8) were fitted in MATLAB (The Mathworks Inc., Natick, MA, USA) using an unconstrained non-linear minimization to yield the smallest absolute difference between measurements and fitted function. The measurements used for the fitting of a function for a phantom thickness $t$ placed at a $d=d^{\text {ref }}=50 \mathrm{~cm}$ were performed for square fields with sizes $3 \mathrm{~cm} \mathrm{x}$ $3 \mathrm{~cm}$ to $24 \mathrm{~cm} \times 24 \mathrm{~cm}$. 

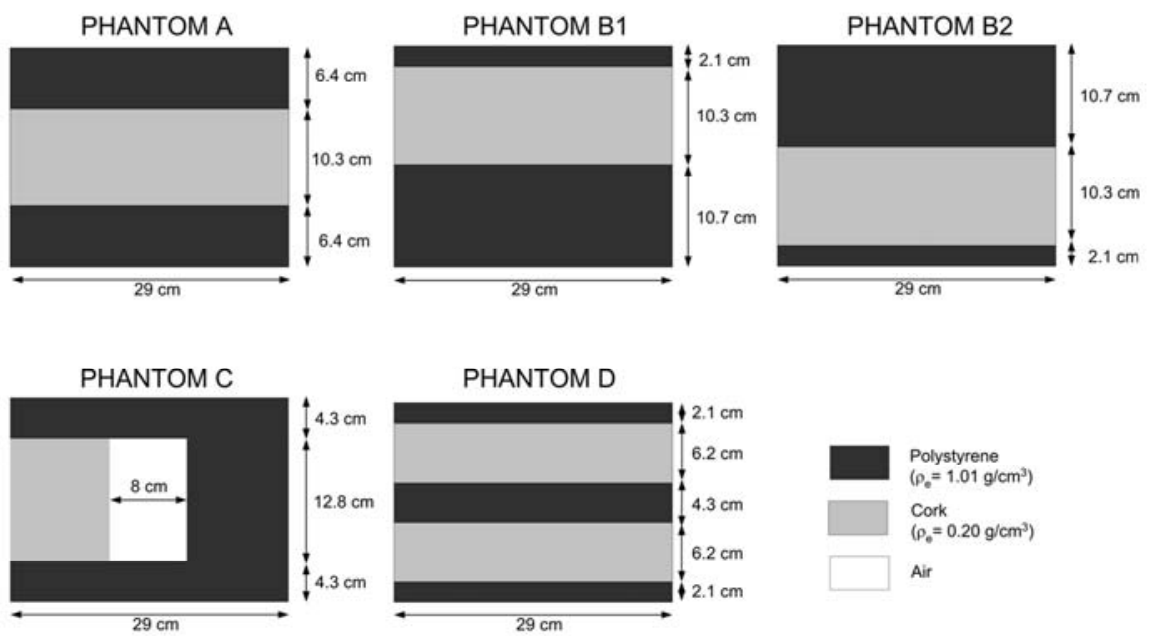

Figure 4.2: Characteristics of the phantoms used in the inhomogeneous phantom study. These phantoms are constructed of stacked layers of polystyrene and cork with an area of $29 \mathrm{~cm} \times 29$ $\mathrm{cm}$ having various thicknesses.

To verify the kernel functions for other distances than $d^{\text {ref }}$, the $8.6 \mathrm{~cm}$ PS phantom was placed at $d=45.7$ and $54.3 \mathrm{~cm}$, and the $17.1 \mathrm{~cm}$ PS phantom at $d=41.4$ and $58.6 \mathrm{~cm}$.

\subsubsection{Homogeneous phantoms}

In order to test the accuracy of the portal dose prediction model for homogeneous phantoms ionization chamber measurements were performed at on- and off-axis locations $0,3,6$, and $9 \mathrm{~cm}$ along the longest side of a $5 \mathrm{~cm} \mathrm{x} 24 \mathrm{~cm}$ and a $15 \mathrm{~cm} \times 24$ $\mathrm{cm}$ field (field sizes are expressed at the isocenter plane). The phantoms were placed with their midplane coinciding with the isocenter, resulting in a midplane-to-detector distance of $d=50 \mathrm{~cm}$.

The accuracy of a PDI predicted for an asymmetric field was investigated for two phantoms with thicknesses of 8.6 and $17.1 \mathrm{~cm}$ PS. A field size of $7.5 \mathrm{~cm} \times 24 \mathrm{~cm}$ (X1=0 $\mathrm{cm}, \mathrm{X} 2=7.5 \mathrm{~cm}, \mathrm{Y} 1=12 \mathrm{~cm}$, and $\mathrm{Y} 2=12 \mathrm{~cm}$ ) was used for these measurements applying the linear detector array.

The limitations of the model imposed by assuming that the center of the phantom is located at the isocenter was investigated by placing the phantom with its top or bottom side at the isocenter plane, resulting in a smaller and larger midplane-to-detector distance. 2D dose measurements were done with the detector array for two phantom thicknesses: $8.6 \mathrm{~cm}$ and $17.1 \mathrm{~cm}$ PS. The resulting midplane-to-detector distances $d$ were 45.7, 50.0, and $54.3 \mathrm{~cm}$ and 41.5, 50.0, and $58.6 \mathrm{~cm}$ for the 8.6 and $17.1 \mathrm{~cm}$ thick phantoms, respectively. For the predictions of the PDIs we assumed that the phantom was positioned symmetrically around the 
isocenter at $d=50.0 \mathrm{~cm}$, and only the radiological thickness was used without further information about the location of the phantom.

\subsubsection{Inhomogeneous phantoms}

Inhomogeneous phantoms are placed with their geometrical midplane coinciding with the isocenter plane. The inhomogeneous phantoms can be divided into midplane symmetric and midplane asymmetric phantoms. For the midplane symmetric phantoms the radiological midplane coincides with the isocenter plane, for the midplane asymmetric phantoms the radiological midplane is shifted relative to the geometrical midplane. The inhomogeneous phantoms, constructed from PS and cork $\left(\rho_{\text {cork }}=0.20 \mathrm{~g} / \mathrm{cm}^{3}\right)$ are schematically shown in Fig. 4.2 . The field size used to irradiate the phantoms was $15 \mathrm{~cm} \times 24 \mathrm{~cm}$.

Phantoms A, B1, and B2 all have the same geometrical and radiological thickness and consist of a slab of cork embedded in two layers of PS. For phantom A the radiological midplane is the same as the geometrical midplane, while for phantom B1/B2 the radiological midplane is $2.3 \mathrm{~cm}$ shifted towards/away from the detector, respectively.

Phantom $\mathrm{C}$ is a midplane symmetric phantom consisting of a slab of cork, air and PS positioned between two layers of PS. In the center, the air gap has an $8 \mathrm{~cm}$ width while on the left and right side there is a slab of PS and cork, respectively.

Phantom D consists of 2 slabs of cork of $6.2 \mathrm{~cm}$ having on top and below a layer of 2.1 $\mathrm{cm}$ PS, and between the two slabs of cork a $4.3 \mathrm{~cm}$ layer of PS has been positioned.

\subsubsection{Clinical case study}

The prediction of a PDI for a clinical treatment situation has been made to demonstrate the feasibility of the model. The radiological thickness has been calculated by ray-tracing through the patient's CT-scan. A tangential breast field was used for treatment of this patient with a field size of $11.1 \mathrm{~cm} \times 16.2 \mathrm{~cm}$. The PDI has been predicted based on the radiological thickness map of the patient and the PDI without the patient in the beam.

\subsubsection{Data presentation}

Transmission measurements are expressed as a scatter-to-primary ratio $(S P R)$, while differences between measured and calculated $S P R$ are expressed as a residual scatter-to-primary ratio ( $r S P R)$.

To verify the model, PDIs were measured with and without the objects in the beam. The PDI without object was normalized to 1000 a.u. at the center of the field. The PDI with object was taken relative to this normalized open beam dose value by performing a point measurement on the central axis of the field with and without the object.

We compared predicted dose distributions, $I^{P}$, with measured dose distributions, $I^{M}$. Results are expressed relative to the central field $I_{C}{ }^{M}$ dose as a mean $\left(I^{P}-I^{M}\right) / I_{C}{ }^{M} \pm$ one standard deviation (SD). Differences between extracted radiological thicknesses, $t^{E}$, 


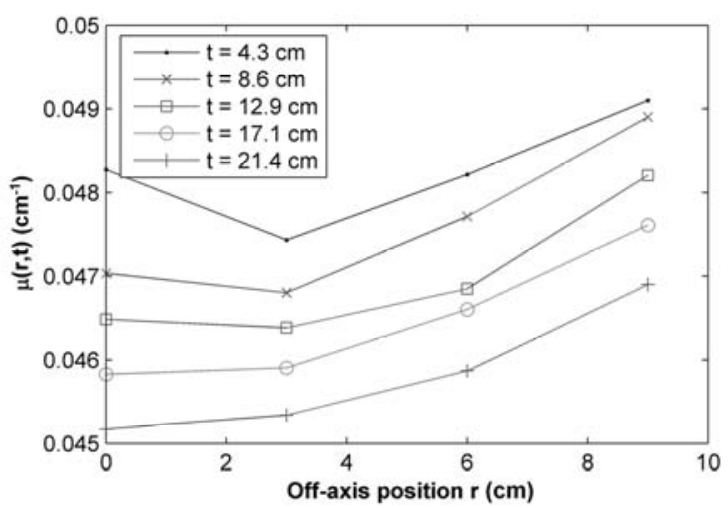

Figure 4.3: Effective attenuation coefficient $\mu$ as a function of off-axis distance $r$ and phantom thickness $t$.

and actual radiological thicknesses, $t^{A}$, are expressed as an absolute difference $\left(t^{E}-t^{A}\right) \pm 1$ $\mathrm{SD}$. Absolute differences in radiological thickness are related to differences in primary portal dose by the attenuation coefficient. Distances in the Figs. 4.5 and 4.7-4.9 are expressed at the EPID plane and the distances in the Figs. 4.3, 4.4, and 4.6 are expressed at the isocenter.

For the clinical case, the differences between predicted and measured PDIs have been quantified using the gamma evaluation approach. ${ }^{33}$ Gamma is smaller than 1 for dose differences $<3 \%$, relative to the maximum dose of the PDI measured, or spatial differences $<5 \mathrm{~mm}$.

\subsection{RESULTS}

\subsubsection{Derivation of the model parameters}

\subsubsection{Effective attenuation coefficient}

The effective attenuation coefficient $\mu\left(r_{(x, y)}, t_{(x, y)}\right)$ as a function of the off-axis distance $r$ and phantom thickness $t$ is shown in Fig. 4.3. The effective attenuation coefficient increases with off-axis distance (beam softening) and decreases with phantom thickness (beam hardening).

\subsubsection{Pencil beam scatter kernels}

In Fig. 4.4 the measured and fitted on-axis scatter-to-primary ratios (SPR) are shown along with the residual scatter-to-primary ratios $(r S P R)$ between measurement and fit. The fitted coefficients of all kernel functions are shown in Table 4.1. The fitted Gaussian pencil beam scatter kernel values for various phantom thicknesses and different midplane-to-detector distances are graphically shown in Fig. 4.5. The SPR 

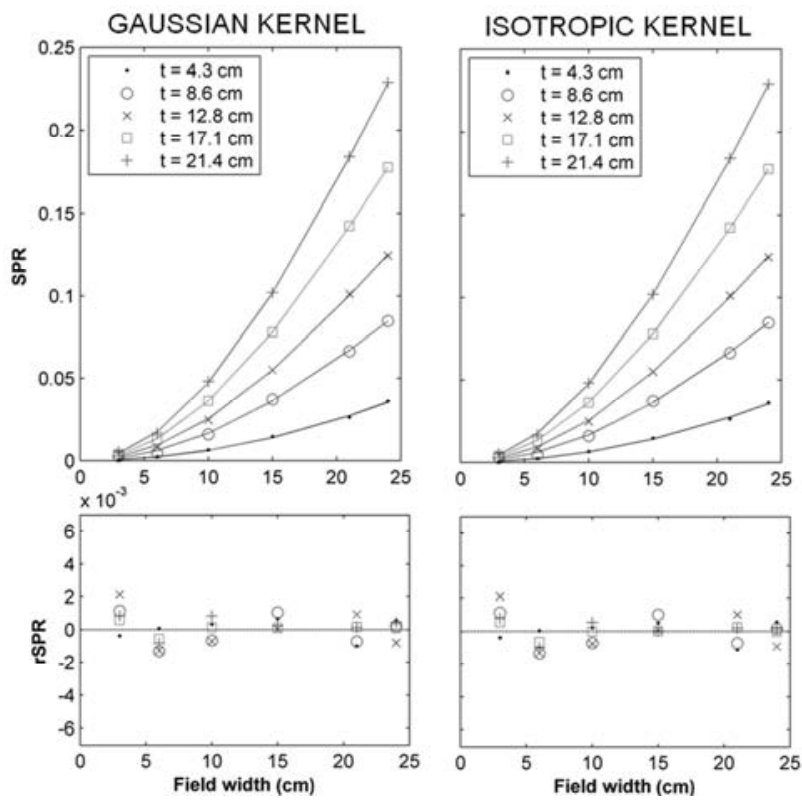

\section{KLEIN-NISHINA KERNEL}
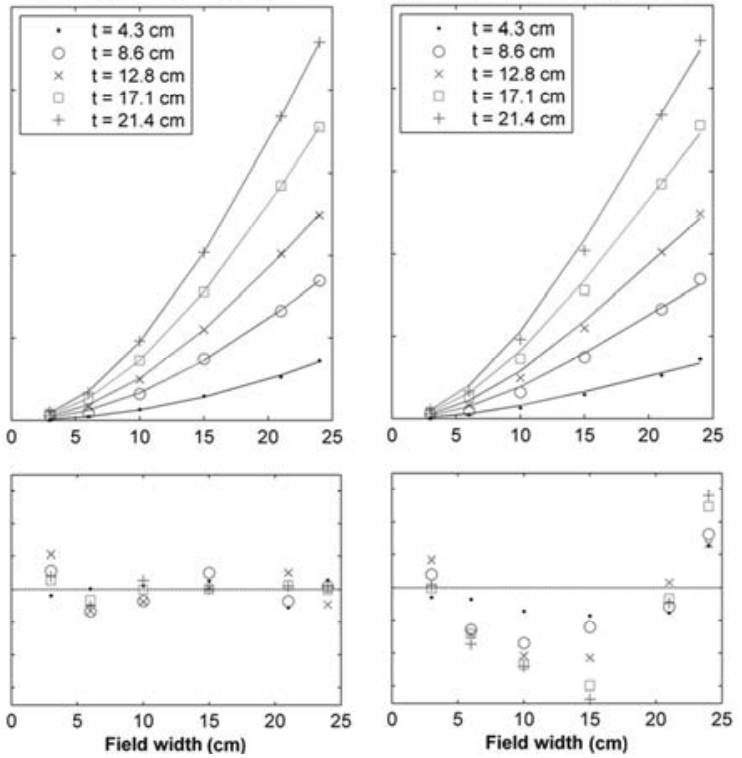

Figure 4.4: Fitted and measured SPR values for various field sizes and phantom thicknesses for the three kernel functions with a midplane-to-detector distance of $50.0 \mathrm{~cm}$. Symbols indicate measured SPR data and the lines represent calculated SPR values. The bottom plots show the residual SPR.

Table 4.1: Fitted parameters for the three pencil beam scatter kernel functions. The reference distance dref for the calculation of these parameters is $50 \mathrm{~cm}$.

\begin{tabular}{|c|c|c|c|c|c|}
\hline \multirow{2}{*}{$\begin{array}{l}\text { Polystyrene } \\
\text { thickness } \\
\qquad \begin{array}{c}t \\
(\mathrm{~cm})\end{array}\end{array}$} & \multicolumn{2}{|c|}{ Gaussian kernel } & \multicolumn{2}{|c|}{ Isotropic kernel } & \multirow{2}{*}{$\begin{array}{c}\text { Klein-Nishina } \\
\text { kernela } \\
c_{1}^{\text {ref }}(t) \\
\left(10^{-2}\right)\end{array}$} \\
\hline & $\begin{array}{c}c_{1}^{r e f}(t) \\
\left(10^{-5} \mathrm{~cm}^{-2}\right)\end{array}$ & $\begin{array}{c}c_{2}{ }^{r e f}(t) \\
(\mathrm{cm})\end{array}$ & $\begin{array}{l}c_{1}^{\text {ref }}(t) \\
\left(10^{-2}\right)\end{array}$ & $\begin{array}{c}c_{2}^{r e f}(t) \\
(\mathrm{cm})\end{array}$ & \\
\hline 4.3 & 2.24 & 147.15 & 25.08 & 104.93 & 7.81 \\
\hline 8.6 & 5.04 & 36.57 & 9.13 & 42.42 & 15.53 \\
\hline 12.8 & 6.41 & 32.45 & 9.05 & 37.47 & 19.11 \\
\hline 17.1 & 7.56 & 32.32 & 10.40 & 36.93 & 22.50 \\
\hline 21.4 & 8.29 & 30.56 & 10.04 & 34.60 & 24.25 \\
\hline
\end{tabular}

a The average photon energy E for the Klein-Nishina kernel function [Eq. (8)] is kept fixed at $2 \mathrm{MeV}$ representing a $6 \mathrm{MV}$ photon beam. 

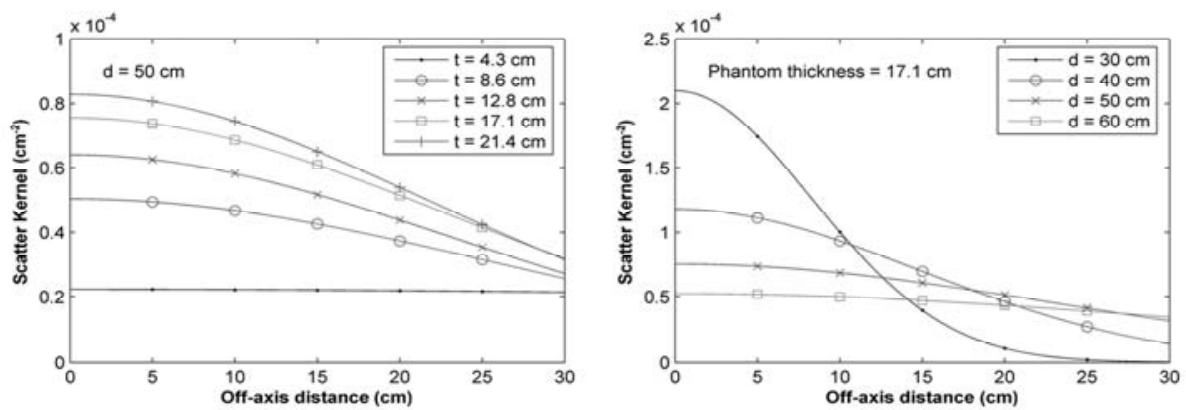

Figure 4.5: Gaussian pencil beam scatter kernels. The left figure shows scatter kernels for five phantom thicknesses with one midplane-to-detector distance of $50.0 \mathrm{~cm}$. The right figure shows scatter kernels for four midplane-to-detector distances and a phantom thickness of $17.1 \mathrm{~cm}$. Kernels are fitted for the situation with $d=d^{\text {ref }}=50.0 \mathrm{~cm}$.

could be fitted with high accuracy in case of the Gaussian and the isotropic kernel. The deviations $(r S P R)$ from the actual SPR were small: $8 \cdot 10^{-4}$ (1 SD) for these two kernels. The Klein-Nishina kernel showed somewhat larger systematic deviations: $3 \cdot 10^{-3}$ (1 SD) between fitted and measured SPR values.

The measured SPR values for the symmetric field widths of 4 and $5 \mathrm{~cm}$ were not used in the calculation of the scatter kernel, to avoid any bias in fitting non-equidistant spaced field sizes. The deviations between measured and calculated SPR values for these field sizes were, however, comparable to those for the other field sizes.

The width of the Gaussian and isotropic kernel shows a decrease for larger phantom thicknesses, while the Klein-Nishina kernel function has only one fit parameter and a constant width determined by the average incident photon energy. A decrease in width due to beam hardening for larger phantom thicknesses could be incorporated in the Klein-Nishina kernel function to correct for this increase in average energy.

Figure 4.6 shows the $S P R$ for the $17.1 \mathrm{~cm}$ phantom for midplane-to-detector distances other than the fitting geometry with $d=d^{\text {ref }}=50 \mathrm{~cm}$. Differences in $S P R$ for both the 8.6 $\mathrm{cm}$ (not shown) and the $17.1 \mathrm{~cm}$ phantom for the Gaussian and the isotropic kernel did not exceed $4 \cdot 10^{-3}$ (excluding the measurement point for the $24 \mathrm{~cm} \times 24 \mathrm{~cm}$ field). Values calculated using the Klein-Nishina kernel showed deviations up to $1 \cdot 10^{-2}$ from the measurements. The Gaussian kernel showed a slightly better modeling for larger fields than the isotropic kernel. This may be explained by the faster fall-off of the Gaussian function compared to the isotropic kernel function. The Gaussian kernel showed the best agreement between measured and fitted scattered transmission.

\subsubsection{Homogeneous phantoms}

Based on the accuracy assessment of the pencil beam scatter kernels as described in the previous section, all PDI predictions were made using the Gaussian set of pencil beam scatter kernels. 

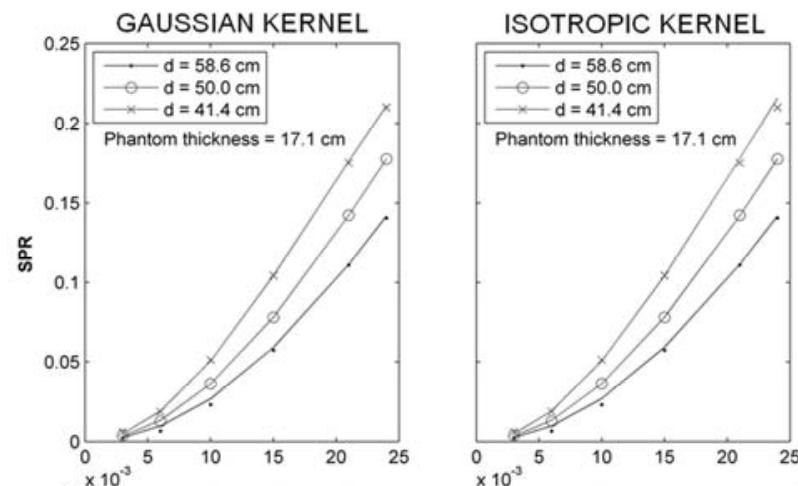

\section{KLEIN-NISHINA KERNEL}
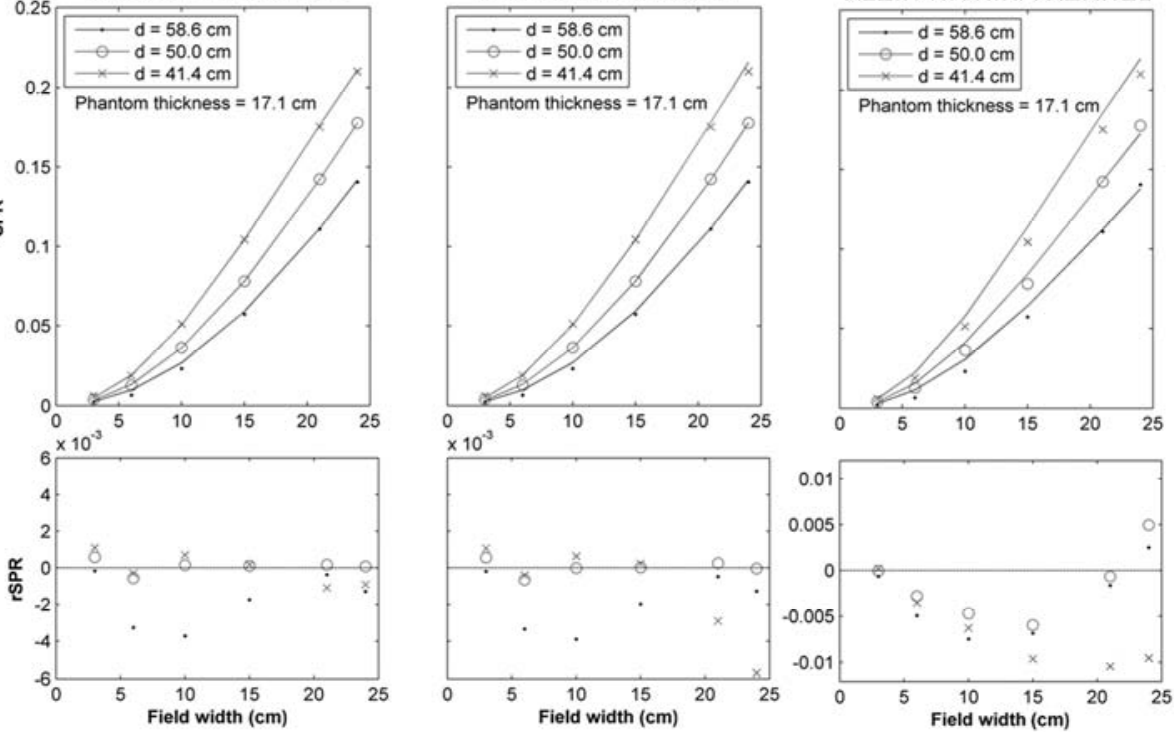

Figure 4.6: Measured and calculated SPR values for a $17.1 \mathrm{~cm}$ polystyrene phantom for different midplane-to-detector distances. Symbols indicate measured SPR data and lines represent calculated SPR values. The bottom plots show the residual SPR.

\begin{tabular}{|c|c|c|c|c|c|}
\hline \multirow[t]{2}{*}{ Phantom } & $d$ & $\begin{array}{c}\text { Mean } \\
\text { dose } \\
\text { difference } \\
\pm 1 \mathrm{SD}\end{array}$ & $\begin{array}{l}\text { Maximum } \\
\text { dose } \\
\text { difference }\end{array}$ & $\begin{array}{l}\text { Mean } \\
\text { thickness } \\
\text { difference } \\
\pm 1 \text { SD }\end{array}$ & $\begin{array}{l}\text { Maximum } \\
\text { thickness } \\
\text { difference }\end{array}$ \\
\hline & $(\mathrm{cm})$ & (\%) & $\min \ldots \max (\%)$ & $(\mathrm{mm})$ & $\min \ldots \max (\mathrm{mm})$ \\
\hline Homogeneous & 45.7 & $+0.3 \pm 0.3$ & $-0.6 \ldots+1.3$ & $+0.7 \pm 0.8$ & $-1.5 \ldots+3.1$ \\
\hline \multirow[t]{2}{*}{$8.6 \mathrm{~cm}$ PS } & 50.0 & $+0.9 \pm 0.3$ & $+0.0 \ldots+1.8$ & $+2.0 \pm 0.7$ & $-0.1 \ldots+4.3$ \\
\hline & 54.3 & $+2.0 \pm 0.3$ & $+0.9 \ldots+2.6$ & $+4.8 \pm 0.7$ & $+2.4 \ldots+6.4$ \\
\hline \multirow{3}{*}{$17.1 \mathrm{~cm}$ PS } & 41.5 & $-1.2 \pm 0.8$ & $-3.1 \ldots+0.9$ & $-3.7 \pm 2.0$ & $-8.5 \ldots+2.1$ \\
\hline & 50.0 & $+1.5 \pm 0.5$ & $-0.1 \ldots+2.7$ & $+4.0 \pm 1.2$ & $0.0 \ldots+7.3$ \\
\hline & 58.6 & $+2.7 \pm 0.6$ & $+1.4 \ldots+4.7$ & $+7.3 \pm 1.5$ & $+3.8 \ldots+12.5$ \\
\hline
\end{tabular}

The differences between predicted and measured dose values for the homogeneous phantoms, both for the on- and off-axis points, were small: $0.2 \pm 0.3 \%$ ( $1 \mathrm{SD}$ ). The 
extraction of the radiological thickness for these measurements was not pursued because only point measurements were made, while for the calculation of the radiological thickness a full 2D portal dose has to be measured.

The accuracy was also assessed for an asymmetric field. The predicted dose distribution was within $0.9 \pm 0.3 \%$ and $1.1 \pm 0.5 \%$ ( 1 SD) of the measured dose distribution for the 8.6 and 17.1 thick cm PS phantom, respectively. For these two phantoms the radiological thickness was in addition extracted and was within $2.1 \pm 0.8$ $\mathrm{mm}$ and $2.9 \pm 1.2 \mathrm{~mm}(1 \mathrm{SD})$ of the actual radiological thickness.

The model was further tested in configurations that deviated from the reference geometry; i.e., at midplane-to-detector distances other than $d=d^{r e f}=50 \mathrm{~cm}$. The results of these measurements are shown in Table 4.2. The differences between predicted and measured dose values for the reference geometry are comparable to the results for the asymmetric field: $0.9 \pm 0.3 \%$ and $1.5 \pm 0.5 \%$ (1 SD) for the 8.6 and $17.1 \mathrm{~cm}$ thick phantom, respectively. The influence of the midplane-to-detector distance on the accuracy of the model can be best demonstrated for the $17.1 \mathrm{~cm}$ PS phantom and is shown in Fig. 4.7. If the phantom is placed towards the detector $(d=41.5 \mathrm{~cm})$, there is an under-prediction of the dose of $-1.2 \pm 0.8 \%$ (1 SD). Displacement of the phantom away from the detector $(d=58.6 \mathrm{~cm})$ results in an over-prediction of $2.7 \pm 0.6 \%$ (1 SD). These differences are also reflected in the extraction of the radiological thickness. Displacement of the phantom towards/away from the detector results in a lower/higher extracted radiological thickness of $-3.7 \pm 2.0 \mathrm{~mm}$ and $7.3 \pm 1.5 \mathrm{~mm}$ (1 SD), respectively.

This effect of midplane-to-detector distance on portal dose prediction can be taken into account in the parameter $d$ of the scatter kernels. If scatter kernels calculated for

midplane-to-detector distances of 41.5 and $58.6 \mathrm{~cm}$ were used for the prediction of the PDIs at these distances, the differences between predicted and measured dose values were much reduced: $1.0 \pm 0.5 \%(d=58.6 \mathrm{~cm})$ and $0.3 \pm 0.6 \%(d=41.5 \mathrm{~cm})(1 \mathrm{SD})$, for the $17.1 \mathrm{~cm}$ PS phantom, compared to $2.7 \pm 0.6 \%$ and $-1.2 \pm 0.8 \%$ (1 SD) for the original kernels at $d=d^{\text {ref }}=50 \mathrm{~cm}$.

\subsubsection{Inhomogeneous phantoms}

The results of the inhomogeneous phantom study are shown in Table 4.3. The differences between predicted and measured dose values for phantoms $A$ and $D$ were small $0.1 \pm 0.4 \%$ and $0.0 \pm 0.4 \%(1 \mathrm{SD})$, respectively. Differences between extracted and measured radiological thickness were also small: $0.2 \pm 1.0 \mathrm{~mm}$ and $0.0 \pm 1.0 \mathrm{~mm}$ (1 SD) for phantoms $A$ and $D$, respectively, with a maximum difference between extracted and actual radiological thickness of $3.2 \mathrm{~mm}$.

Phantoms B1 and B2 have the same radiological thickness as phantom A but the geometrical midplane differs from the radiological midplane. This distance between the two midplanes is $2.3 \mathrm{~cm}$. This is reflected in the differences between predicted and measured dose for phantoms B1 and B2. For phantom B1 the radiological midplane is shifted towards the portal plane relative to the geometrical 

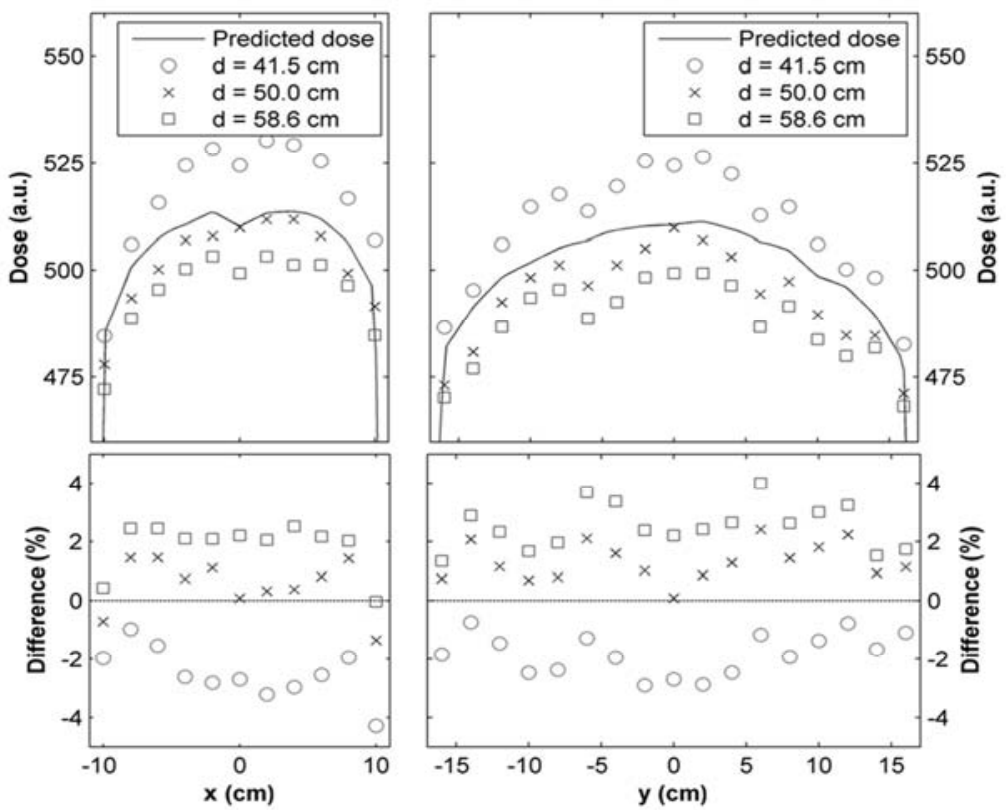

Figure 4.7: Portal dose profiles behind a homogeneous phantom of $17.1 \mathrm{~cm}$ polystyrene thickness with different midplane-to-detector distances. Differences are expressed relative to the dose on the central beam axis. Left and right plots show the horizontal and vertical cross section through the central beam axis, respectively. The symbols indicate the dose points measured with the linear detector array and the solid lines represent the predicted dose. Predictions are based on a midplane-to-detector distance of $50.0 \mathrm{~cm}$.

\begin{tabular}{|c|c|c|c|c|}
\hline \multirow[t]{2}{*}{$\begin{array}{l}\text { Inhomogeneous } \\
\text { phantom }\end{array}$} & $\begin{array}{c}\text { Mean } \\
\text { dose } \\
\text { difference } \\
\pm 1 \text { SD }\end{array}$ & $\begin{array}{l}\text { Maximum } \\
\text { dose } \\
\text { difference }\end{array}$ & $\begin{array}{l}\text { Mean } \\
\text { thickness } \\
\text { difference } \\
\pm 1 \mathrm{SD}\end{array}$ & $\begin{array}{l}\text { Maximum } \\
\text { thickness } \\
\text { difference }\end{array}$ \\
\hline & $(\%)$ & $\min \ldots \max (\%)$ & $(\mathrm{mm})$ & $\min \ldots \max (\mathrm{mm})$ \\
\hline Phantom A & $+0.1 \pm 0.4$ & $-1.1 \ldots+1.2$ & $+0.2 \pm 1.0$ & $-2.7 \ldots 3.2$ \\
\hline Phantom B1 & $-0.4 \pm 0.4$ & $-1.7 \ldots+0.9$ & $-1.1 \pm 1.1$ & $-4.5 \ldots 2.2$ \\
\hline Phantom B2 & $+0.9 \pm 0.5$ & $-0.4 \ldots+2.2$ & $+2.3 \pm 1.2$ & $-0.9 \ldots 5.6$ \\
\hline Cork & $+0.7 \pm 0.4$ & $-0.3 \ldots+1.3$ & $+3.1 \pm 1.9$ & $-1.4 \ldots 5.9$ \\
\hline \multirow[t]{2}{*}{ Phantom C } & $-0.4 \pm 0.3$ & $-1.1 \ldots+0.7$ & $-0.9 \pm 0.8$ & $-2.7 \ldots 1.6$ \\
\hline & $-0.9 \pm 0.5$ & $-2.2 \ldots+0.1$ & $-2.4 \pm 1.2$ & $-6.1 \ldots 0.2$ \\
\hline Phantom D & $0.0 \pm 0.4$ & $-1.4 \ldots+1.1$ & $0.0 \pm 1.0$ & $-3.2 \ldots 2.6$ \\
\hline
\end{tabular}



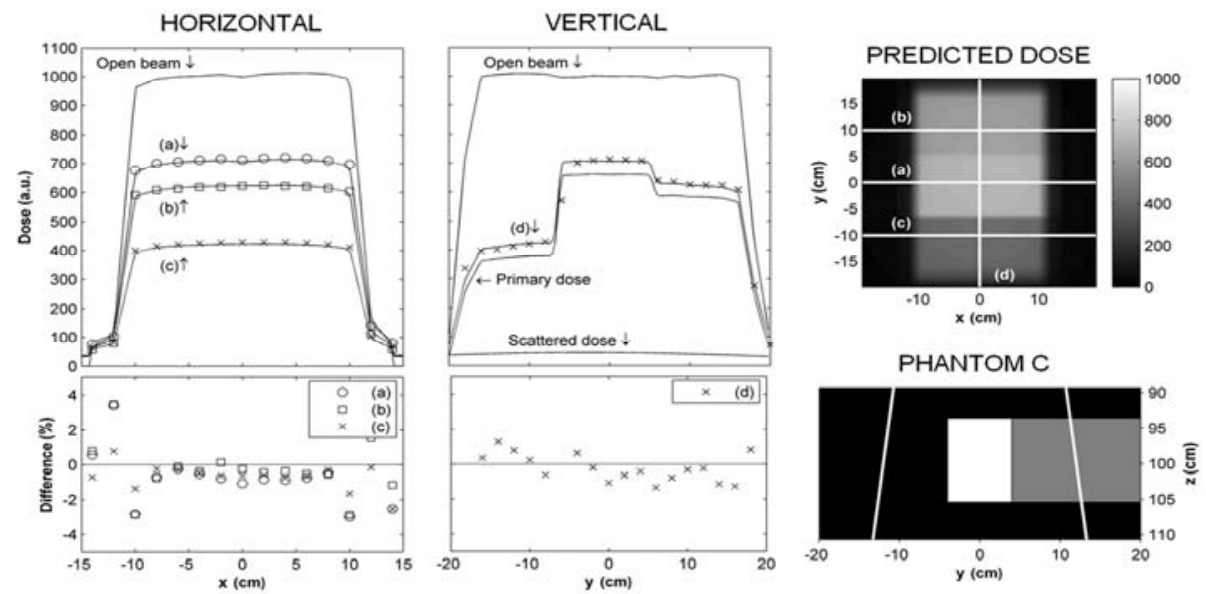

Figure 4.8: Portal dose profiles behind inhomogeneous phantom C. Measured and predicted portal dose profiles are plotted along with the difference between measurement and prediction in the left and middle figure. The symbols indicate the measured dose profile and the solid lines are the predicted dose profiles. In the top middle figure the open beam dose profile as well as the predicted primary and scattered dose profiles are given. The phantom is schematically shown in the bottom right figure, in which the superimposed white lines indicate the beam edges. The top right figure shows the predicted PDI.

midplane. There was a small underprediction of $-0.4 \pm 0.4 \%$ (1 SD). For phantom B2 the opposite effect occurred: An overprediction of $0.9 \pm 0.5 \%$ (1 SD). This result was also visible in the extraction of the radiological thickness. An underestimation of the radiological thickness of $-1.1 \pm 1.1 \mathrm{~mm}(1 \mathrm{SD})$ for phantom B1 and an overestimation of $2.3 \pm 1.2 \mathrm{~mm}$ (1 SD) for phantom B2 was observed.

The results for phantom $\mathrm{C}$ have been divided into the three sections: Behind the cork, the air or the polystyrene slab. Differences between prediction and measurement were again smaller than $1.0 \%$ (mean) and the thickness extraction was within $3 \mathrm{~mm}$ (mean) of the actual thickness. In Fig. 4.8 the predicted PDI is shown with three horizontal and one vertical cross section.

\subsubsection{Clinical case study}

The predicted PDI was compared with the PDI measured during the actual treatment. The results of the gamma evaluation are presented in Fig. 4.9 , showing that $87 \%$ of the gamma values in the breast are below 1 , indicating a dose difference smaller than $3 \%$. However, there are some regions with a higher gamma value. For instance, there is a rather large deviation at the coordinate $(x, y)=(-2,-8)$. This difference might be due to a different arm position during treatment relative to planning CT. Also some differences are seen at the border of the lung, which might be a breathing artifact. The larger error outside the patient area in the portal dose image might be due to the EPID calibration procedure. 

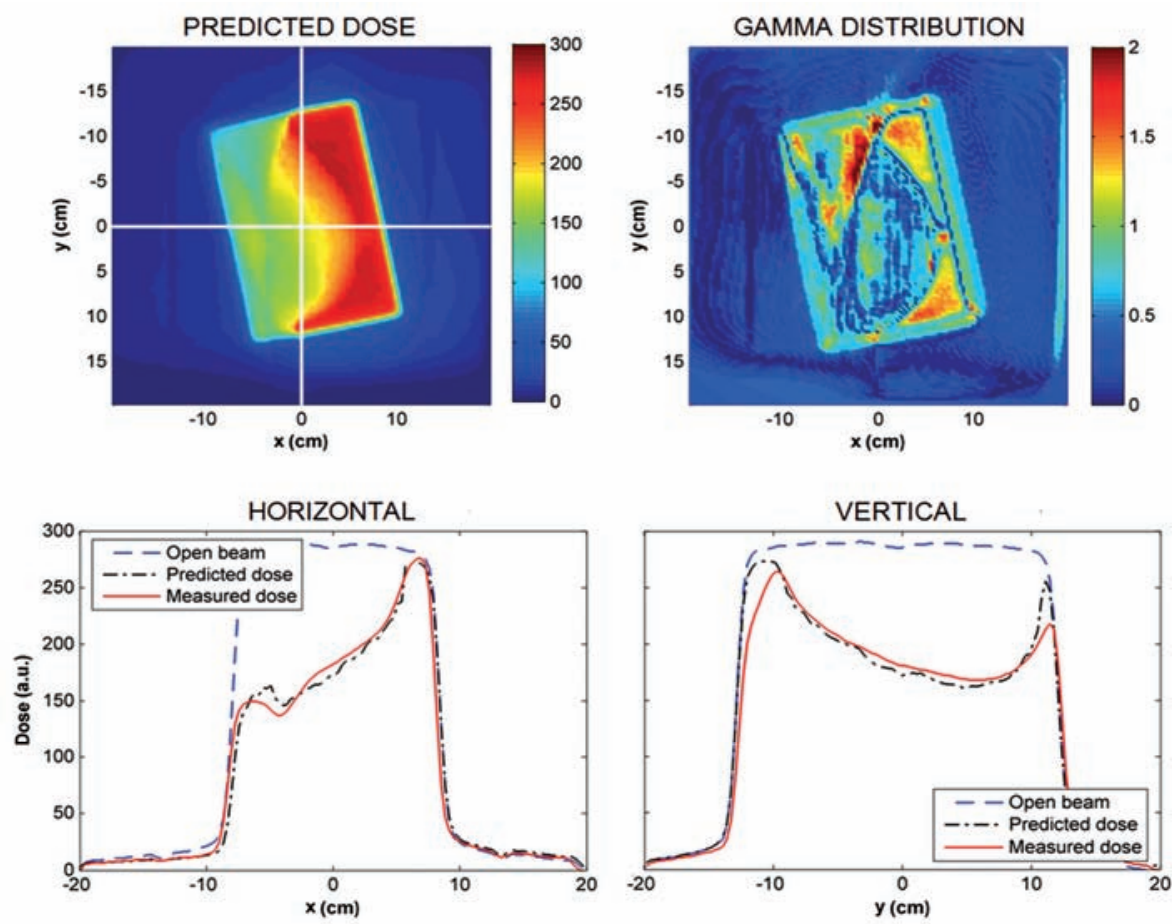

Figure 4.9: Comparison of a predicted and measured portal dose image during a breast cancer treatment. The top left figure shows the predicted dose distribution. The top right figure shows the gamma evaluation for a $3 \%$ dose difference and a $5 \mathrm{~mm}$ spatial difference criterion. The bottom left and right figure show a horizontal and a vertical cross section of the measured and predicted dose, as well as the dose without the patient in the beam (open beam dose).

\subsection{DISCUSSION}

\subsubsection{Input parameters of the model}

The derivation of an (off-axis) effective attenuation coefficient has shown to be an adequate way to describe the primary dose behind a phantom. Differences up to $8 \%$ in the effective attenuation coefficient due to beam hardening or off-axis beam softening have to be taken into account. For accurate primary dose prediction these differences are incorporated into the model.

Kernels are required to predict the phantom scattered dose in a portal dose image that consists of a primary and phantom scattered dose component. An advantage of fitting the pencil beam scatter kernels to the pre-defined functions is that the fitting procedure only has to be done for one midplane-to-detector distance. Other distances can be taken into account by the scaling factor $s$ in Eqs. (4.6)-(4.8), which limits the set 
of measurements needed to calculate scatter kernels. This is an advantage compared to the numerical calculation of the scatter kernels as described by Pasma et al. ${ }^{10}$

A comparison with Monte Carlo generated kernels was not made because in order to do this properly, the Monte Carlo kernels have to be calculated under the same conditions as the fitted kernels. Comparison with published data of Monte Carlo simulations of scatter kernels ${ }^{24,29}$ is, therefore, not meaningful, while in our institution such a code is not available.

A detailed analytical derivation of the pencil beam scatter kernels has been given by Spies et al. ${ }^{25,30}$ Their model is rather elaborate and describes the scatter from first principles. For the Klein-Nishina kernel we presented a simplified model that describes the first-order Compton scattering from the midplane of the phantom based on a single mean energy. This simplified model did not show accurate predictions for the set-up we investigated. A contribution from higher-order scattering maybe needs to be incorporated for this model to be more accurate. The replacement of a kernel based on a single mean energy with a kernel that is weighted over the energy spectrum of the linac could maybe also improve the accuracy. Another possibility is to take an effective scatter source point into account that is not located in the midplane of the phantom. We did, however, not compare our method with the analytical model of Spies et al. $^{25,30}$ in a quantitative way. The ultimate comparison for portal dose predictions models is with measured portal dose values, which was the purpose of this study.

\subsubsection{Portal dose prediction model}

Our portal dose prediction model is based on the portal dose distribution without an object in the beam and the radiological thickness of the object. The radiological thickness is assumed to be centered symmetrically around the isocenter plane. The phantom study indicates that the differences are generally smaller than $2 \%$ (mean) if this criterion is met. However, if the radiological midplane does not coincide with the isocenter plane a slightly larger deviation between the predicted and the actual measured portal dose is observed. The difference is the result of a variation in scatter contribution in the portal plane. In our model we assume that scatter is produced at the center-of-mass of a column of material along a ray line with the center-of-mass at the isocenter. If this center-of-mass is displaced, then the scatter contribution from a pencil beam can be scaled with divergence (inverse square law). ${ }^{24}$ For the prediction of the portal dose this distance of detector to the center-of-mass of a column of phantom material can be taken into account using the EHP concept as introduced by Pasma and colleagues. ${ }^{10}$ The derivation of these pencil beam scatter kernels is only necessary for one midplane-to-detector distance, because phantom scatter at other midplane-to-detector distances can be derived by taking into account the scaling factor $s$ in Eqs. (4.6)-(4.8). Both the radiological thickness and the distance of the center-of-mass to the detector can be calculated from the CT scan of the patient.

Inhomogeneities can also be taken into account by using the EHP concept. Inhomogeneous phantoms are replaced by homogeneous phantoms with their 
radiological thickness distributed symmetrically around the radiological midplane. Our phantom study indicates that the assumptions of the prediction and extraction model are correct: highly inhomogeneous phantoms can be replaced by their corresponding radiological thickness distributed around the radiological midplane.

In clinical treatment situations differences between predicted and measured PDIs may be difficult to interpret. Kroonwijk et al. ${ }^{34}$ showed that inter-fraction organ motion in prostate cancer treatment could be responsible for such a difference. However, how this difference at the position of the EPID will affect the dose distribution inside the patient was difficult to estimate. Therefore, a relation between portal dose and patient dose has to be made. 2D exit- and midplane reconstructions were developed but these techniques do not take into account a possible different patient anatomy between planning CT and treatment. With the use of a CT scan that is made prior to the treatment or a cone-beam CT acquired while the patient is on the treatment table, the actual patient anatomy is known and a 3D dose reconstruction can be performed completely independent of the planning data. Such a dose reconstruction technique will gain more insight in the actual dose delivered to a patient during a treatment, which is the ultimate aim of our EPID dosimetry project.

If we compare our model to other models presented in the literature, some remarks can be made. Our model is able to predict the portal dose values with differences between predicted and measured values below $2 \%$ (mean difference, SD below $1 \%$ ) if the object is midplane symmetric. A slightly higher accuracy was achieved by Pasma et al. ${ }^{10}$ who found differences of $1 \%$ (1 SD). These findings are both in agreement by a phantom study of McCurdy et al., ${ }^{12}$ who validated the use of the pencil beam algorithm and the EHP concept against full Monte Carlo simulations. The differences between their Monte Carlo simulations and the pencil beam prediction model, for an air gap between detector and patient larger than $10 \mathrm{~cm}$, were smaller than $3 \%$ (maximum difference).

A difference of our model compared to other models is that our predictions and measurements are done under full scatter conditions in a water tank. We also calibrated our EPID under these conditions. The input for the model is a PDI measured without the object in the beam where lateral scatter in the portal plane also contributes to the measurement point. The possible different amount of lateral scatter behind objects is inherently taken into account during the derivation of the pencil beam scatter kernels; these kernels describe both the amount of patient or phantom scatter and the reduced amount of lateral scatter.

\subsubsection{Radiological thickness extraction}

The first step of a 3D dose reconstruction method is an extraction of the primary portal dose from a measured PDI, which is related to the radiological thickness. The radiological thickness can be extracted solely on the basis of a PDI with and without an object while no a priori information of the object in the beam is needed. This was done to make the primary portal dose extraction completely independent of the object in the beam. The extraction model assumes that the thickness of the object is distributed 
symmetrically around the isocenter plane. Deviations from this criterion lead to an approximation of the extracted radiological thickness. If this criterion is met then the thickness extraction is within $7 \mathrm{~mm}$ (maximum) from the actual thickness, which corresponds to a primary portal dose difference of $3 \%$. The primary portal dose is needed for a 3D dose reconstruction method based on back-projection. The primary portal dose, as defined in Eq. (4.1), also contains some lateral scatter from inside the EPID because the input parameter of the model is $O(x, y)$, a PDI without the object in place, obtained under full scatter conditions. For the back-projection it is necessary to remove this EPID scatter contribution from the primary portal dose if this quantity is used for back-projection.

The assumption that the radiological midplane coincides with the isocenter plane is for clinical treatment situations generally not valid. However, normally the isocenter is placed at the center of the tumor so large deviations from the fitting geometry are not expected for deep-seated tumors. In addition a different midplane-to-detector distance only affects the calculation of the patient scattered dose at the position of the EPID. This dose distribution is only a small part of the total dose distribution, because scatter-to-primary portal dose ratios for clinical treatments with the EPID at $150 \mathrm{~cm}$ from the focus are of the order of $5 \%-20 \%$.

Pasma et al. $^{28}$ used this application of the model to estimate the radiological thickness of compensators and showed that for these large distances the model could be simplified by using the inverse square law to extend to the distance of a small subset of the kernels with the largest airgap to the distance of the compensator for scatter predictions. The scatter signal for this geometry is much reduced, only $4 \%$ of the total signal, due to the large air gap between compensator and portal imager. This is not the case for actual treatments, so the proposed iterative technique should be used for calculating the radiological thickness at these smaller air gaps.

\subsubsection{Other considerations}

Another application of our portal dose prediction model is also possible: The reconstruction of the open beam portal dose based on the radiological thickness and the portal dose measured with an object in the beam. ${ }^{31}$ With this application the dose distribution of the incident beam can be checked, which can be a valuable tool for checking beam fluence in IMRT treatments. This application of the model is not explored in this study, but its implementation is similar to the thickness extraction procedure.

Furthermore, application of the extraction of the primary dose from portal images can be used in the reconstruction of a megavoltage cone-beam CT scan. If these scatter corrected portal images are used, a better estimation of the reconstructed electron densities is possible and cupping artifacts are reduced, as described by Spies et al. ${ }^{32}$

However, the most important application that needs to be developed is the 3D dose reconstruction based on the back-projection of the primary portal dose to an actual model of the patient during treatment. ${ }^{21}$ This will be the focus of our future work. 


\subsection{CONCLUSIONS}

The portal dose prediction model described in this study shows good agreement between predicted and measured portal dose values. It can, therefore, be concluded that fitting the phantom scattered component of transmission measurements to a pre-defined Gaussian kernel function with a two parameter fit, is an easy and accurate way of deriving pencil beam scatter kernels that can be used for accurate portal dose prediction. The accuracy of the model for the phantom study is better than $2 \%$ (mean difference) if the radiological midplane of the irradiated object coincides with the isocenter plane. If this assumption is no longer valid, larger deviations will occur, which can, however, be corrected. With our model it is also possible to extract the radiological thickness with an accuracy better than $7 \mathrm{~mm}$ (maximum difference) from the actual thickness, which is related to primary portal dose differences smaller than $3 \%$. In conclusion, a valuable and versatile tool for the analysis of portal dose images has been presented and can be used for many applications for quality control of patient treatments. This work is an important step towards the ultimate aim of this project: checking the actual 3D dose distribution inside the patient during treatments with advanced irradiation techniques. 


\section{REFERENCES}

1 M.G. Herman, J.J. Kruse and C.R. Hagness, "Guide to clinical use of electronic portal images," J. Appl. Clin. Med. Phys. 1, 38-57 (2000).

2 B.J.M. Heijmen, K.L. Pasma, M. Kroonwijk, V.G.M. Althof, J.C.J. de Boer, A.G. Visser, and H. Huizenga, "Portal dose measurement in radiotherapy using an electronic portal imaging device (EPID)," Phys. Med. Biol. 40, 1943-1955 (1995).

3 K.L. Pasma, M. Kroonwijk, J.C.J. de Boer, A.G. Visser, and B.J.M. Heijmen, "Accurate portal dose measurement with a fluoroscopic electronic portal imaging device (EPID) for open and wedged beams and dynamic multileaf collimation," Phys. Med. Biol. 43, 2047-2060 (1998).

4 B. Warkentin, S. Steciw, S. Rathee, and B.G. Fallone, "Dosimetric IMRT verification with a flat-panel EPID," Med. Phys. 30, 3143-3155 (2003).

5 S.M.J.J.G. Nijsten, A.W.H. Minken, P. Lambin, and I.A.D. Bruinvis, "Verification of treatment parameter transfer by means of electronic portal dosimetry," Med. Phys. 31, 341-347 (2004).

6 M. Essers and B.J. Mijnheer, "In vivo dosimetry during external photon beam radiotherapy," Int. J. Radiat. Oncol., Biol., Phys. 43, 245-259 (1999).

7 G.J. Meijer, A.W.H. Minken, K.M. van Ingen, B. Smulders, H. Uiterwaal, and B.J. Mijnheer, "Accurate in vivo dosimetry of a randomized trial of prostate cancer irradiation," Int. J. Radiat. Oncol., Biol., Phys. 49, 1409-1418 (2001).

8 E. Bloemen-van Gurp, W. du Bois, P. Visser, I.A.D. Bruinvis, D. Jalink, J. Hermans, and P. Lambin, "Clinical dosimetry with MOSFET dosimeters to determine the dose along the field junction in a split beam technique," Radiother. Oncol. 67, 351-357 (2003).

9 R. Bogaerts, A. Van Esch, R. Reymen, and D. Huyskens, "A method to estimate the transit dose on the beam axis for verification of dose delivery with portal images," Radiother. Oncol. 54, 39-46 (2000).

10 K.L. Pasma, B.J.M. Heijmen, M. Kroonwijk, and A.G. Visser, "Portal dose image (PDI) prediction for dosimetric treatment verification in radiotherapy. I. An algorithm for open beams," Med. Phys. 25, 830-840 (1998).

11 K.L. Pasma, S.C. Vieira, and B.J.M. Heijmen, "Portal dose image prediction for dosimetric treatment verification in radiotherapy. II. An algorithm for wedged beams," Med. Phys. 29, 925-931 (2002).

12 B.M.C. McCurdy and S. Pistorius, "Photon scatter in portal images: accuracy of a fluence based pencil beam superposition algorithm," Med. Phys. 27, 913-922 (2000).

13 R. Boellaard, M.B. van Herk, and B.J. Mijnheer, "A convolution model to convert transmission dose images to exit dose distributions," Med. Phys. 24, 189-199 (1997).

14 R. Boellaard, M.B. van Herk, H. Uiterwaal, and B.J. Mijnheer, "Twodimensional exit dosimetry using a liquid-filled electronic portal imaging device and a convolution model," Radiother. Oncol. 44, 149-157 (1997).

15 D. Huyskens, J. Van Dam, and A. Dutreix, "Midplane dose determination using in vivo dose measurements in combination with portal images," Phys. Med. Biol. 39, 1089-1101 (1994).

16 R. Boellaard, M. Essers, M.B. van Herk, and B.J. Mijnheer, "New method to obtain the midplane dose using portal in vivo dosimetry," Int. J. Radiat. Oncol., Biol., Phys. 41, 465-474 (1998).

17 R. Boellaard, M.B. van Herk, H. Uiterwaal, and B.J. Mijnheer, "First clinical tests using a liquid-filled electronic portal imaging device and a convolution model for the verification of the midplane dose," Radiother. Oncol. 47, 303-312 (1998). 
18 V.N. Hansen, P.M. Evans, and W. Swindell, "The application of transit dosimetry to precision radiotherapy," Med. Phys. 23, 713-721 (1996).

19 M. Partridge, M. Ebert, and B.M. Hesse, "IMRT verification by threedimensional dose reconstruction from portal beam measurements," Med. Phys. 29, 1847-1858 (2002).

20 R.J.W. Louwe, E.M.F. Damen, M.B. van Herk, A.W.H. Minken, O. Törzsök, and B.J. Mijnheer, "Three-dimensional dose reconstruction of breast cancer treatment using portal imaging," Med. Phys. 30, 2376-2389 (2003).

21 J. Pouliot, J. Chen, O. Morin, M. Svatos, F. Ghelmansarai, M. Mitschke, M. Aubin, P. Xia, C. Chuang, K. Bucci, M. Roach, III, P. Hernandez, Z. Zheng, D. Hristov, A. Bani-Hashemi, and L. Verhey, "Dose-Guided Radiation Therapy with Megavoltage Cone-Beam CT," in "Program and Abstract book 8th International Workshop on Electronic Portal Imaging," University of Sussex, Brighton, UK, pp. 16-19 (2004).

22 D.A. Jaffray, J.J. Battista, A. Fenster, and P. Munro, "X-ray scatter in megavoltage transmission radiography: physical characteristics and influence on image quality," Med. Phys. 21, 45-60 (1994).

23 W. Swindell and P. M. Evans, "Scattered radiation in portal images: a Monte Carlo simulation and a simple physical model," Med. Phys. 23, 63-73 (1996).

24 V.N. Hansen, W. Swindell, and P.M. Evans, "Extraction of primary signal from EPIDs using only forward convolution," Med. Phys. 24, 1477-1484 (1997).

25 L. Spies, P.M. Evans, M. Partridge, V.N. Hansen, and T. Bortfeld, "Direct measurement and analytical modeling of scatter in portal imaging," Med. Phys. 27, 462-471 (2000).

26 J. Pouliot, A. Bani-Hashemi, J. Chen, M. Svatos, F. Ghelmansarai, M. Mitschke, M. Aubin, P. Xia, O. Morin, K. Bucci, M. Roach, III, P. Hernandez, Z. Zheng, D. Hristov, and L. Verhey, "Low-dose megavoltage cone-beam CT for radiation therapy," Int. J. Radiat. Oncol., Biol., Phys. 61, 552-560 (2005).

27 D.A. Jaffray, J.H. Siewerdsen, J.W. Wong, and A.A. Martinez, "Flatpanel cone-beam computed tomography for image-guided radiation therapy," Int. J. Radiat. Oncol., Biol., Phys. 53, 1337-1349 (2002).

28 K.L. Pasma, M. Kroonwijk, E.B. van Dieren, A.G. Visser, and B.J.M. Heijmen, "Verification of compensator thicknesses using a fluoroscopic electronic portal imaging device," Med. Phys. 26, 1524-1529 (1999).

29 B.M.C. McCurdy and S. Pistorius, "Photon scatter in portal images: physical characteristics of pencil beam kernels generated using the EGS Monte Carlo code," Med. Phys. 27, 312-320 (2000).

30 L. Spies and T. Bortfeld, "Analytical scatter kernels for portal imaging at $6 \mathrm{MV}$," Med. Phys. 28, 553-559 (2001).

31 L. Spies, M. Partridge, B.A. Groh, and T. Bortfeld, "An iterative algorithm for reconstructing incident beam distributions from transmission measurements using electronic portal imaging," Phys. Med. Biol. 46, N203-211 (2001).

32 L. Spies, M. Ebert, B.A. Groh, B.M. Hesse, and T. Bortfeld, "Correction of scatter in megavoltage cone-beam CT," Phys. Med. Biol. 46, 821-833 (2001).

33 D.A. Low, W.B. Harms, S. Mutic, and J.A. Purdy, "A technique for the quantitative evaluation of dose distributions," Med. Phys. 25, 656-661 (1998).

34 M. Kroonwijk, K.L. Pasma, S. Quint, P.C.M. Koper, A.G. Visser, and B.J.M. Heijmen, "In vivo dosimetry for prostate cancer patients using an electronic portal imaging device (EPID); demonstration of internal organ motion," Radiother. Oncol. 49, 125-132 (1998). 


\section{CHAPTER}

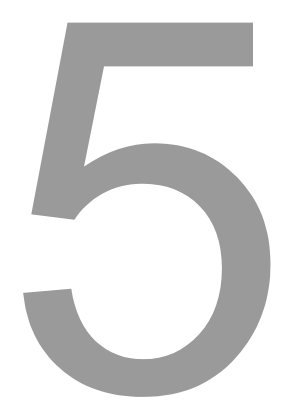

A global calibration model for $a$-Si EPIDs used for transit dosimetry

Med. Phys. 34(10), 3872-3884 (2007)

S.M.J.J.G. Nijsten W.J.C. van Elmpt M. Jacobs B.J. Mijnheer A.L.A.J. Dekker

P. Lambin A.W.H. Minken 



\section{ABSTRACT}

Electronic portal imaging devices (EPIDs) are not only applied for patient set-up verification and detection of organ motion but are also increasingly used for dosimetric verification. The aim of our work is to obtain accurate dose distributions from a commercially available amorphous silicon ( $a$-Si) EPID for transit dosimetry applications. For that purpose, a global calibration model was developed, which includes a correction procedure for ghosting effects, field size dependence and energy dependence of the $a$-Si EPID response. In addition, the long-term stability and additional buildup material for this type of EPID were determined. Differences in EPID response due to photon energy spectrum changes have been measured for different absorber thicknesses and field sizes, yielding off-axis spectrum correction factors based on transmission measurements. Dose measurements performed with an ionization chamber in a water tank were used as reference data, and the accuracy of the dosimetric calibration model was determined for a large range of treatment conditions. Gamma values using $3 \%$ as dose-difference criterion and $3 \mathrm{~mm}$ as distance-to-agreement criterion were used for evaluation. The field size dependence of the response could be corrected by a single kernel, fulfilling the gamma evaluation criteria in case of virtual wedges and intensity-modulated radiation therapy fields. Differences in energy spectrum response amounted up to $30 \%-40 \%$, but could be reduced to less than $3 \%$ using our correction model. For different treatment fields and (in)homogeneous phantoms, transit dose distributions satisfied in almost all situations the gamma criteria. We have shown that $a$-Si EPIDs can be accurately calibrated for transit dosimetry purposes. 


\subsection{INTRODUCTION}

Several types of electronic portal imaging devices (EPIDs) have been developed but only video-based, scanning liquid-filled ionization chamber and indirect amorphous silicon ( $a$-Si) type EPIDs have evolved into commercially available systems. These EPIDs were originally designed for geometric verification of patient set-up during treatment but have also been used for monitoring organ motion and multileaf collimator (MLC) accuracy. ${ }^{1-3}$ It was soon realized, however, that EPIDs could also be used for dosimetric verification, both pre-treatment and during treatment. ${ }^{4-7} \mathrm{~A}$ prerequisite for dose verification with EPIDs is an accurate knowledge of the EPID response for different treatment conditions to implement a dosimetric calibration model to convert a grayscale EPID image into a portal dose image (PDI).

The behavior of $a$-Si EPIDs is currently investigated by a number of groups but mainly for two commercially available EPIDs: The aS500/1000 EPID (Varian Medical Systems, Palo Alto, CA $)^{8-10}$ and the iView GT EPID (Elekta, Crawley, United Kingdom). ${ }^{11,12}$ Dosimetric characteristics like linearity of detector response, reproducibility, ghosting, and field size dependence have been reported frequently, but the variation in response due to energy spectrum changes is often not taken into account.

To use $a$-Si EPIDs for transit dosimetry, differences in energy spectrum response between EPID and ionization chamber are very important. Amorphous silicon EPIDs are oversensitive for low energy photons ${ }^{13}$ and will, therefore, measure incorrect portal dose distributions if no correction is applied. Greer ${ }^{14}$ showed off-axis differences in response up to $20 \%$ for the Varian aS500 EPID using $6 \mathrm{MV}$ photon beams but only in-field and without an object in the beam. Several authors described the use of Monte Carlo (MC) simulations to investigate the energy dependence of the same EPID behind (in)homogeneous phantoms ${ }^{15,16}$ and for MLC-blocked fields. ${ }^{17}$ McCurdy et al. ${ }^{15}$ and Li et al. $^{17}$ investigated the accuracy of MC-based algorithms to predict portal dose images, accounting for energy dependence. Kirkby et al. ${ }^{16}$ examined the specific case of beam hardening along the central beam axis by using compensator material, and showed that the discrepancy between open and attenuated beam calibration can be as high as $8 \%$ for $6 \mathrm{MV}$ photon beams. Hence, an energy dependence correction is needed in case of a changing photon beam energy spectrum.

Most publications describe dosimetric characteristics of $a$-Si EPIDs without an object in the beam or for one specific absorber thickness only, ${ }^{5,14,18}$ by taking EPID energy dependencies into account using a single flood field correction. In case of transit dosimetry, this is not sufficient because energies on-axis and off-axis are changing dependent on the size and composition of the object in the beam. For a larger object thickness, this means that the mean photon energy increases due to beam hardening and the EPID signal is overcorrected by the single flood field correction. Because the energy of the beam is lower off-axis, energies will relatively increase more, and the under-response of the EPID will be even larger off-axis. On the other hand, for increasing object thickness more low energy scattered photons will be generated in the object, which will increase the EPID response and will limit the overcorrection by the flood field correction. ${ }^{19}$ Chen et al. ${ }^{20}$ have already described an energy spectrum 
correction model for the same $a$-Si flat panel as used in our work (Perkin-Elmer XRD 1640 AL7). This model was in the form of lookup tables for a small number of on-axis and off-axis detector positions. In addition, the EPID signal had to be scaled with the number of monitor units (MUs) before a correction factor could be determined. Furthermore, only single frame acquisitions were used limiting the maximum number of monitor units to 3-4 to prevent detector saturation. The accuracy of the calibration was 3\% (2 SD) for the in-field region using homogeneous and inhomogeneous phantoms. In the out-of-field regions, local differences ranged from $-10 \%$ to as much as $65 \%$.

The aim of this work was to develop a global calibration model for $a$-Si EPIDs taking into account all relevant EPID response corrections to obtain full-scatter PDIs suitable for transit dosimetry applications. The model should be based on measurements only and include an energy spectrum correction model independent of beam parameters such as the number of MUs. Furthermore, the calibration model had to be accurate out-of-field. The calibration model has been applied to three new commercially available $a$-Si EPIDs.

\subsection{MATERIALS AND METHODS}

\subsubsection{Equipment}

The three EPIDs used in our work are OptiVue 500/1000/1000 ST amorphous silicon flat panel portal imagers (Siemens Medical Solutions, Concord, CA) that are attached to Oncor medical linear accelerators (Siemens Medical Solutions, Concord, CA). For all linear accelerators (LINACS), photon beam energies of 6 and $10 \mathrm{MV}$ have been used which are dosimetrically matched within 1\% (1 SD) looking at depth dose curves, beam profiles, and output factors. Each LINAC is equipped with a multileaf collimator existing of 82 leaves and a virtual wedge option. The virtual wedge can be applied by moving one of two collimating jaws during irradiation and changing dose rate values to build a wedge profile. The accelerators have two dose rate modes: A low dose rate mode (50 $\mathrm{MU} / \mathrm{min}$ for both photon energies) and a high dose rate mode (300 MU/min for $6 \mathrm{MV}$ and $500 \mathrm{MU} / \mathrm{min}$ for $10 \mathrm{MV}$ ); the dose rate is changed by varying the pulse repetition frequency.

All imagers have an active imaging area of $41 \mathrm{~cm} \times 41 \mathrm{~cm}$ consisting of $512 \times 512$ pixels (OptiVue 500) or $1024 \times 1024$ pixels (OptiVue 1000) yielding a pixel resolution of 0.8 and $0.4 \mathrm{~mm}$, respectively. The distance to the imager can vary between 115.0 and $160.0 \mathrm{~cm}$, while lateral and longitudinal movements of the panels are not possible. We investigated the dosimetric characteristics of one OptiVue 500 EPID and two OptiVue 1000 EPIDs. One of the two OptiVue 1000 EPIDs has an increased signal-to-noise ratio due to a larger $a$-Si layer thickness and can be used for megavoltage cone-beam CT acquisition (OptiVue 1000 ST). The OptiVue EPIDs are used in the free running acquisition mode for all measurements, which means that imaging starts with beam-on and stops when the beam turns off. Acquisition software is the 
Siemens Coherence Therapist Workspace software, version 1.0.657 (OptiVue 500 and OptiVue 1000) and version 2.0.125 (OptiVue 1000 ST). An OptiVue EPID image is stored as a two-dimensional (2D) accumulated grayscale value distribution averaged over all subframes; the individual subframes are not stored by the acquisition software. The accumulated image is automatically corrected for individual pixel sensitivity, beam profile, dead pixels, and dark current by the acquisition software. The correction for individual pixel sensitivity and beam profile is done in one single step using flood field images, which have to be acquired periodically for each beam energy, dose rate, and source-to-detector distance (gain correction). Dead pixel maps are generated by the system interactively, correcting bad pixel values by setting them to the mean value of neighboring pixels. A dark current correction is done dynamically every $30 \mathrm{~s}$ for different integration times (offset correction). For the iView GT EPID, similar corrections are done and have been described by Louwe et $a .^{12}$ in more detail. For the Coherence Therapist Workspace version 1.0.657, the residual offset was 256 grayscale values per subframe, and this had to be corrected during dosimetric calibration. The integration time per subframe is fixed to $285 \mathrm{~ms}$ (OptiVue 500 and 1000) and $145 \mathrm{~ms}$ (OptiVue 1000 ST) resulting in constant frame acquisition rates of $\approx 3.5$ and $\approx 6.9$ frames per second, respectively. During all the measurements described in our work, the source-to-detector distance was $150 \mathrm{~cm}$, and all automatic corrections by the acquisition software were maintained. The accumulated grayscale value distributions were multiplied by the number of subframes to obtain the grayscale value distribution $G_{\text {raw. }}$.

Reference point dose measurements were performed with a calibrated ionization chamber (Thimble chamber CC13, Scanditronix Wellhöfer, Schwarzenbruck, Germany) in combination with an electrometer (35040 Keithley, Cleveland, OH). A CA24 multidetector ionization chamber array and a MD240 multichannel electrometer (Scanditronix Wellhöfer, Bartlett, TN) were used for 2D relative dose measurements. The array consists of 23 waterproof vented thimble ionization chambers; a single chamber's design is identical to the CC13 chamber. All dose measurements were done in water using the Blue Phantom water phantom system (Scanditronix Wellhöfer, Bartlett, TN), which has scanning dimensions of $48 \mathrm{~cm} \times 48 \mathrm{~cm} \times 41 \mathrm{~cm}$. The distance between accelerator focus and water surface was set to $145 \mathrm{~cm}$ with the point of measurement at a depth of $5 \mathrm{~cm}$; this depth was chosen to ensure electron equilibrium and to reduce electron contamination effects.

Analysis of the measurements and the implementation of the dosimetric calibration model is done in the programming language MATLAB 7.1 (The MathWorks Inc., Natick, MA).

\subsubsection{Open field calibration}

The dosimetric calibration model consists of two steps. First, dosimetric EPID characteristics like long-term stability, buildup, ghosting effects, and field size dependence have been investigated in order to implement a dosimetric calibration for open fields. The energy dependency of the EPID without an object in the beam is taken into account using a single flood field correction. ${ }^{14}$ Second, the model is extended for 
transit dosimetry applications including an energy spectrum correction model that corrects differences in EPID response between measurements with and without an object in the beam (Sec. 5.2.3). For all measurements, the relative EPID response is defined as the grayscale value $G_{\text {raw }}$ in a region of interest (ROI) of $0.5 \mathrm{~cm} \times 0.5 \mathrm{~cm}$ divided by the portal dose $D_{p}$ measured with the thimble ionization chamber at $5 \mathrm{~cm}$ depth in water on the central beam axis unless mentioned otherwise. In-field corresponds to the region in a portal dose distribution within the treatment field including the penumbra region (dose values $\geq 20 \%$ of the maximum dose); out-of-field is outside the penumbra region (dose values $<20 \%$ of the maximum dose).

\subsubsection{Long-term stability}

To determine the long-term stability of the EPID response, portal images have been acquired every morning during a period of about 6 months for all EPIDs. The two photon beam energies and a field size of $10 \mathrm{~cm} \times 10 \mathrm{~cm}$ were used delivering 100 MUs. The mean grayscale value has been measured within a ROI of $0.5 \mathrm{~cm} \times 0.5 \mathrm{~cm}$ and was corrected for machine monitor output variations with time. The long-term reproducibility is expressed as the standard deviation of the grayscale values with respect to the mean grayscale value over the measurement period.

\subsubsection{Buildup}

The OptiVue EPIDs have an intrinsic $1 \mathrm{~mm}$ aluminum plate covering the phosphor layer. This plate provides some buildup for the photons and absorbs low energy, scattered radiation that reduces image quality. For (portal) dosimetry, it is important to measure beyond dose maximum and to attenuate the low energy photons scattered from a patient to maximum extent because $a$-Si EPIDs are oversensitive for these low energy photons. ${ }^{13}$ Therefore, an additional copper $(\mathrm{Cu})$ plate of $3 \mathrm{~mm}$ has been placed directly on the EPID as proposed by McDermott et al. ${ }^{11}$ and the decrease in EPID over-response for one of the OptiVue EPIDs has been compared to the findings of these authors. Residual differences in energy dependence of the EPID response will be eliminated by separate corrections.

The airgap between a $20 \mathrm{~cm}$ polystyrene slab phantom and the EPID was varied to generate different photon scatter distributions at the EPID plane. Airgaps of 25, 30, 40, 50,60 , and $70 \mathrm{~cm}$ were used by changing the position of the phantom because the SDD was fixed to $150 \mathrm{~cm}$. Relative EPID response values were measured for the OptiVue 500 for both photon energies, with and without the copper plate of $3 \mathrm{~mm}$. A field size of $10 \mathrm{~cm} \times 10 \mathrm{~cm}, 100 \mathrm{MUs}$ and the high dose rate mode were used. The dose in water was measured with the CC13 ionization chamber on the central beam axis. To verify that image quality does not decrease dramatically due to additional buildup material, the critical frequency $f_{50}$ has been measured with the QC-3V phantom (Standard Imaging, Middleton, WI) ${ }^{21}$ for the $6 \mathrm{MV}$ photon energy. The additional $3 \mathrm{~mm}$ $\mathrm{Cu}$ buildup plate has been used for all EPIDs during all subsequent measurements in our work. 


\subsubsection{Image lag and ghosting}

Amorphous silicon EPIDs suffer from image lag and ghosting artifacts as described by several authors. ${ }^{11,22,23}$ Ghosting effects for the Siemens OptiVue EPIDs used in our work have been quantified and recently published. ${ }^{22}$ Based on these measurements, image lag and ghosting are accounted for by using a ghosting correction factor $G$ as a function of irradiation time: ${ }^{11}$

$$
G\left(t_{\text {rad }}\right)=1-\sum_{i=1}^{3} A_{i} \cdot \exp \left(-r_{i} \cdot t_{r a d}\right)
$$

where $A$ and $r$ are amplitudes and decay rates, respectively. The irradiation time $t_{\text {rad }}$ is calculated by multiplying the number of subframes with the fixed integration time. The ghosting correction factor is calculated for the high dose rate mode only but also used for correcting EPID response at the low dose rate mode. Furthermore, portal images were acquired for a $20 \mathrm{~cm} \times 20 \mathrm{~cm}$ treatment field delivering 3 MUs after exposing the OptiVue 1000 ST EPID to a dose of 60 MUs using a $6 \mathrm{~cm} \times 6 \mathrm{~cm}$ field of a $10 \mathrm{MV}$ photon beam. Winkler et al. $^{23}$ showed that the relative response could increase about $5 \%$ for the iView GT EPID under these treatment conditions.

All subsequent measurements described in our work are corrected for image lag and ghosting effects, using the ghosting correction factor, unless mentioned otherwise. No minimum time was defined between two measurements to decrease ghosting effects because this was also not done during the quantification of image lag and ghosting.

\subsubsection{Field size dependence and beam profile correction}

For the three EPIDs used in our work, a field size dependence kernel $K_{F}$ and a beam profile correction $B P$ are derived for both photon energies to obtain the dose in water from a portal image:

$$
D_{p}(x, y)=c_{F}^{-1} \cdot\left(G\left(t_{r a d}\right)^{-1} \cdot G_{r a w}(x, y) \cdot B P(x, y) \cdot F\left(T, A_{F}, x, y\right)\right) \otimes^{-1} K_{F}\left(\sqrt{x^{2}+y^{2}}\right)
$$

where $c_{F}$ is the conversion factor from absolute dose in water to EPID grayscale values (Sec. 5.2.2.5), $F$ is the energy spectrum correction (Sec. 5.2.3), as a function of the transmission $T$ and field area $A_{F}$, and $\otimes^{-1}$ denotes a deconvolution. A schematical overview of the dosimetric calibration model in Eq. (5.2) is shown in Fig. 5.1. The radially symmetric kernel incorporates energy deposition in the phosphor screen, optical photon spreading, and energy deposition in water. The beam profile correction recovers the beam profile in an EPID image that was removed by the gain correction; the latter correction inherently corrects on-axis and off-axis dose differences due to energy dependence of the EPID response without an object in the beam. Therefore, the energy spectrum correction $F$ is equal to 1 for open fields. Both $K_{F}$ and $B P$ are fully based on open field measurements, which means for $F=1$.

For the field size dependence kernel calculation, EPID and ionization chamber measurements are performed for square field sizes of $3 \mathrm{~cm} \times 3 \mathrm{~cm}, 6 \mathrm{~cm} \times 6 \mathrm{~cm}, 10 \mathrm{~cm}$ $\times 10 \mathrm{~cm}, 15 \mathrm{~cm} \times 15 \mathrm{~cm}, 20 \mathrm{~cm} \times 20 \mathrm{~cm}$, and $24.2 \mathrm{~cm} \times 24.2 \mathrm{~cm}$; the latter field size is 
the maximum field size that can be measured at $150 \mathrm{~cm}$ with the EPIDs. The measurements in water are performed with the CC13 ionization chamber on the central beam axis. Furthermore, a $10 \mathrm{~cm} \times 10 \mathrm{~cm}$ field shifted $5 \mathrm{~cm}$ off-axis in cross-line and in-line directions is acquired with the EPID. A corresponding in-line dose profile is measured in water through the center of this treatment field using a scanning ionization chamber; the pixel resolution of the beam profile is about $0.3 \mathrm{~mm}$. For the beam profile correction, 2D images are acquired with the EPID and the fast CA24 multidetector ionization chamber array using a field size of $24.2 \mathrm{~cm} \times 24.2 \mathrm{~cm}$. The pixel resolution for the 2D scans with the multidetector array is $5 \mathrm{~mm}$ and $4 \mathrm{~mm}$ in cross-line and in-line directions, respectively. The high dose rate mode is used.

Because the beam profile correction $B P$ should be known before the field size dependence kernel $K_{F}$ can be derived, and vice versa, an iterative calculation procedure is applied which consists out of four steps.

First, the relative EPID response for the square field sizes is normalized to the response for a $10 \mathrm{~cm} \times 10 \mathrm{~cm}$ field. Equation (5.2) can be simplified by assuming a flat beam profile $(B P=1)$ and for $F=1$. An approximation of kernel $K_{F}$ can then be obtained by solving

$$
\begin{aligned}
& \left(\frac{G_{\text {raw }}}{D_{p}}(c, x=0, y=0)\right)_{\text {norm }} \\
& \approx \iint_{\forall|x|<c, \forall|y|<c} K_{F}\left(\sqrt{x^{2}+y^{2}}\right) d x d y=\iint_{\forall|x|<c, \forall|y|<c} \sum_{i=1}^{N} m_{i} \cdot \exp \left(-\left(\frac{\sqrt{x^{2}+y^{2}}}{\sigma_{i}}\right)^{2}\right) d x d y
\end{aligned}
$$

where $x$ and $y$ are the off-axis positions in a portal image, and $c$ is the square field size. The coefficients $m_{i}$ and $\sigma_{i}$ are the parameters of $N$ Gaussians used to describe the field size dependence kernel $K_{F}$.

Second, an approximation of $B P$ can be found by using $K_{F}$ from Eq. (5.3):

$$
B P(x, y) \approx\left(D_{p}(x, y) \otimes K_{F}\left(\sqrt{x^{2}+y^{2}}\right)\right) / G_{\text {raw }}(x, y)
$$

and dividing by the value on the central beam axis. In Eq. (5.4), $D_{p}$ is the $2 D$ relative portal dose distribution measured with the CA24 multidetector ionization chamber array.

Third, the final field size dependence kernel $K_{F}$ is calculated using Eq. (5.2) and the approximated beam profile correction BP. In this case, the Gaussians describing kernel $K_{F}$ are fitted by using an iterative deconvolution scheme applied to the set of square fields and the asymmetric $10 \mathrm{~cm} \times 10 \mathrm{~cm}$ field. Dose differences on the central beam axis for the square fields are utilized to optimize the parameters of the small peaked Gaussians. The parameters of the broader Gaussians of the kernel can be calculated by using the dose profile differences out-of-field for the asymmetric field. The latter parameters can be determined more accurately now due to the approximated non-flat beam profile correction $B P$. 


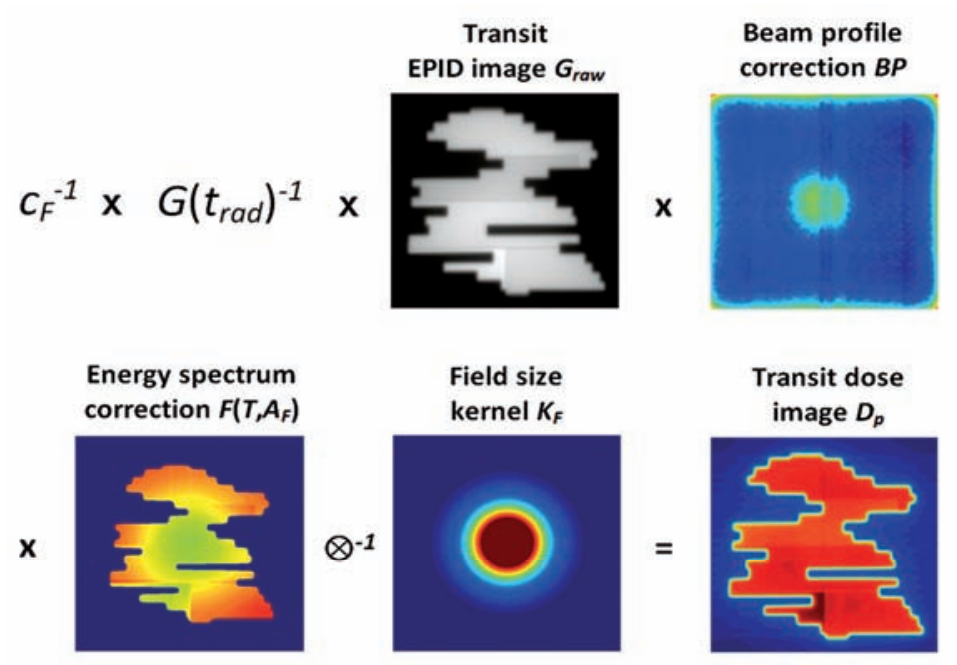

Figure 5.1: Schematical overview of the dosimetric calibration procedure for a transit EPID image. The calibration starts with a grayscale EPID image $G_{\text {raw }}$ that is corrected for possible image lag and ends with a transit dose image $D_{p}$. In case of an open field image, the same procedure applies but the energy spectrum correction $F$ will be equal to 1 for the entire image.

Fourth, Eq. (5.4) is applied again to determine the beam profile correction $B P$ using the final kernel $K_{F}$.

\subsubsection{Absolute dose calibration}

The conversion factor $c_{F}$ is determined for a $10 \mathrm{~cm}$ x $10 \mathrm{~cm}$ open field, $100 \mathrm{MUs}$, and the high dose rate mode for each EPID and photon beam energy separately. On the central beam axis, Eq. (5.2) simplifies to $c_{F}=G^{-1} \cdot G_{\text {raw }}(0,0) /\left.D_{p}(0,0)\right|_{10 \times 10}$ because for the $10 \mathrm{~cm} \times 10 \mathrm{~cm}$ open field the relative EPID response on this location is unchanged by the kernel $K_{F}$, the beam profile correction $B P$ and the energy spectrum correction $F$. The grayscale value $G_{\text {raw }}$ is determined from an EPID image and corrected for image lag and ghosting, using the ghosting correction factor $G$. The absolute portal dose $D_{p}$ is measured with the ionization chamber at a source-to-detector distance of $150 \mathrm{~cm}$ and is corrected for machine output variations.

\subsubsection{Energy spectrum correction}

Photon beam energies at the $a$-Si EPIDs are different in the case of transit dosimetry compared to the situation for an open field (Sec. 5.2.2). Dependent on the entrance photon beam spectrum and the size and composition of the object in the beam, photon beam energies have a different distribution on- and off-axis. Hence, a correction model for differences in energy spectrum should be dependent on off-axis position and phantom/patient thickness. Because the actual change in energy spectrum is difficult to measure directly, the correction model in our work is based on 


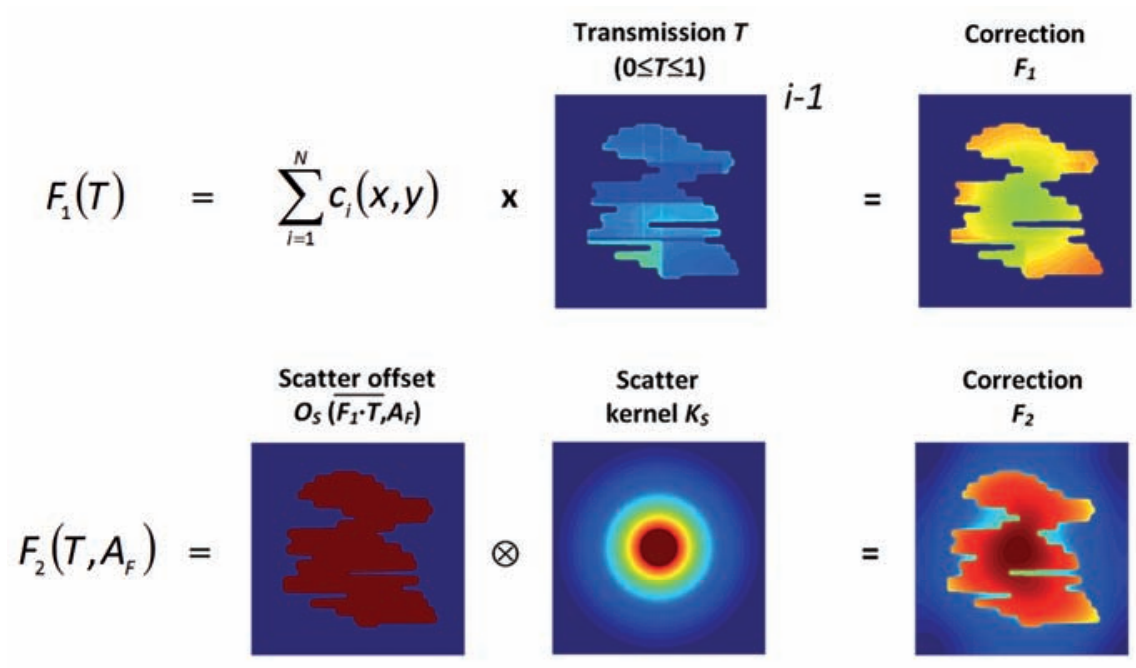

Figure 5.2: Schematical overview of the energy spectrum correction $F$, which is a multiplication of the two individual corrections $F_{1}$ and $F_{2} . F_{1}$ is modeled by a $N-1$ degree polynomial as a function of the transmission $T$ of a beam through a phantom/patient and corrects differences in primary portal dose between measurements with and without an object in the beam. Transmission is defined as the ratio of corresponding pixel values in a transit image and an open field image. Because $F_{1}$ is derived for a field size of $24.2 \mathrm{~cm} \times 24.2 \mathrm{~cm}$, it also corrects for differences in patient scattered dose for this specific field size but is insufficient for smaller field sizes. $F_{2}$ is used to correct for these differences in patient scattered dose and is a function of the mean value of the $F_{1}$-corrected transmission within a treatment field and the field area $A_{F} ;$ a scatter kernel $K_{S}$ is applied to derive the patient scatter correction function $F_{2}$ outside the treatment field. $F_{1}$ is applied only within the treatment field.

the transmission of a beam through a phantom/patient including lateral scatter in the EPID and phantom/patient scatter. The transmission $T$ is calculated as the ratio of grayscale value images $G_{\text {raw }}$ with and without an object in the beam.

Both EPID and dose in water measurements are done using a polystyrene slab phantom with thicknesses of $0,5.2,9.9,20.6,31.0,39.0$, and $44.9 \mathrm{~cm}$, and square field sizes of $3 \mathrm{~cm} \times 3 \mathrm{~cm}, 6 \mathrm{~cm} \times 6 \mathrm{~cm}, 10 \mathrm{~cm} \times 10 \mathrm{~cm}, 15 \mathrm{~cm} \times 15 \mathrm{~cm}, 20 \mathrm{~cm} \times 20 \mathrm{~cm}$, and $24.2 \mathrm{~cm} \times 24.2 \mathrm{~cm}$. The polystyrene slab phantoms are positioned symmetrically around the isocenter plane. Doses in water measurements are performed with the CC13 ionization chamber. Diagonal profiles for a field size of $24.2 \mathrm{~cm} \times 24.2 \mathrm{~cm}$ are scanned at a depth of $5 \mathrm{~cm}$ with a pixel resolution of about $0.3 \mathrm{~mm}$ for all thicknesses. An additional in-line profile is scanned at the same depth for a phantom thickness of $39.0 \mathrm{~cm}$ and a field size of $10 \mathrm{~cm} \times 10 \mathrm{~cm}$. Absolute doses in water measurements are performed at the center of the square treatment fields for all thickness-field size combinations. The measurements are used to derive two individual correction functions $F_{1}$ and $F_{2}$, which have to be multiplied to obtain the overall energy spectrum correction $F$. 
First, the correction $F_{1}$ is derived from the diagonal profiles mainly to correct primary portal dose differences due to differences in energy beam spectrum. Diagonal dose profiles are copied radial symmetrically to obtain $2 \mathrm{D}$ portal dose distributions $D_{p}$, clipping the dose at the field edges of the $24.2 \mathrm{~cm} \times 24.2 \mathrm{~cm}$ treatment field. Outside the field borders, dose values are set to zero, and no effort is made to simulate a penumbra region. A reference grayscale value distribution $G_{r e f}$ is then calculated based on $D_{p}$ :

$$
G_{r e f}(x, y)=c_{F} \cdot D_{p}(x, y) \otimes K_{F}\left(\sqrt{x^{2}+y^{2}}\right)=G\left(t_{r a d}\right)^{-1} \cdot G_{r a w}(x, y) \cdot B P(x, y) \cdot F_{1}(x, y)
$$

where $F_{2}=1$. Corresponding diagonal profiles from the 2D distributions $G^{-1} \cdot G_{\text {raw }} \cdot B P, G_{\text {ref, }}$ and $T$ are used to fit the coefficients of a position dependent $N-1$ degree polynomial $F_{1}$ in a least-squares sense [Fig. 5.2(a)]:

$$
F_{1}(T(r), r)=\frac{G_{r e f}(r)}{G\left(t_{r a d}\right)^{-1} \cdot G_{r a w}(r) \cdot B P(r)}=\sum_{i=1}^{N} c_{i}(r) \cdot T^{i-1}(r)
$$

where $r=\mathrm{V}\left(x^{2}+y^{2}\right) \cdot F_{1}$ mainly corrects for the differences in energy spectrum and is only applied within the treatment field, unless mentioned otherwise. However, $F_{1}$ does also take into account the patient scattered dose differences for the maximum field size of $24.2 \mathrm{~cm} \times 24.2 \mathrm{~cm}$. For other field sizes, a separate correction $F_{2}$ needs to be determined on top of $F_{1}$, which should correct patient scattered dose differences due to differences in irradiated patient volume. For the maximum field size of $24.2 \mathrm{~cm} \mathrm{x}$ $24.2 \mathrm{~cm}, F_{2}$ equals 1 by definition; in case of smaller field sizes, $F_{2}$ will be larger than 1 because less low energy patient scattered photons will be generated and the EPID signal will be underestimated by $F_{1}$.

Second, correction $F_{2}$ is derived from the absolute dose measurements on the central beam axis of the square treatment fields and should satisfy

$$
\left.F_{2}(T(r), c, r)\right|_{C A X}=\left.\frac{D_{p, \text { water }}}{D_{p, E P I D}}\right|_{C A X}=\left.\left(\left\{\left.O_{S}\left(\overline{F_{1}(T(r), r) \cdot T(r), c^{2}}\right)\right|_{\forall r \in f i e l d}\right\} \otimes K_{S}(r)\right)\right|_{C A X}
$$

where $D_{p, E P I D}$ is calculated using Eq. (5.2), and $F=F_{1}$. Correction $F_{2}$ is a $2 \mathrm{D}$ function that is split up in a scatter offset $O_{S}$ and a scatter kernel $K_{S}$ [Fig. 5.2(b)]. Because patient scatter is primarily generated inside the irradiated patient volume, the scatter offset $O_{S}$ is equal to 1 outside a treatment field. Inside the field, $O_{S}$ is dependent on the irradiated patient volume and is therefore a function of the transmission, which is related to the patient thickness and the irradiated field area $A_{F}$. Because patient scatter distributions are relatively flat at the source-to-detector distance of $150 \mathrm{~cm},{ }^{19,24}$ the mean value of the $F_{1}$-corrected transmission within a treatment field is used to derive the scatter offset $O_{S}$. The field area $A_{F}$ is defined as $c^{2}$ for the square treatment fields. The scatter kernel $K_{S}$ is introduced to calculate $F_{2}$ in the out-of-field regions based on the scatter offset $O_{S}$ within a treatment field, because patient scatter also reaches the out-of-field regions. 
First, the kernel $K_{S}$ is calculated by an iterative deconvolution scheme using Eq. (5.7) for a phantom thickness of $39.0 \mathrm{~cm}$ and a field size of $10 \mathrm{~cm} \times 10 \mathrm{~cm}$. Similar to the field size dependence kernel $K_{F}$ in Eq. (5.3), $N$ Gaussians are used to describe the scatter kernel $K_{S}$. In this optimization, $O_{S}$ is set equal to $D_{p \text {, water }} / D_{p, E P I D}$ within the treatment field, and the dose differences between corresponding dose profiles measured in water and with the EPID are minimized in-field only. Second, the values of the scatter offset $O_{S}$ are determined for all absolute dose measurements using $K_{S}$ with the same iterative deconvolution scheme. The values of the scatter offset $O_{S}$ are stored in a lookup table.

For the three EPIDs and two photon energies per LINAC, the parameters in the correction models $F_{1}$ and $F_{2}$ have been calculated. The high dose rate mode was used.

\subsubsection{Verification measurements}

To determine the accuracy of the calibration model, verification measurements have been performed for different treatment conditions using an EPID and the ionization chamber (array). EPID grayscale value images are converted to PDIs and absolute dose profiles from these images have been compared to absolute dose profiles measured with the ionization chamber in water. The field area $A_{F}$ is calculated by using a gradient segmentation technique to determine the field edges in an EPID image. In case of IMRT, portal dose images are calculated per segment using Eq. (5.2) and then summed. Gamma values $^{25,26}$ are calculated using $3 \%$ as dose-difference criterion and $3 \mathrm{~mm}(4.5$ $\mathrm{mm}$ at $150 \mathrm{~cm}$ ) as distance-to-agreement criterion. We used the $3 \%$ dose-difference criterion not only for the local dose differences (local gamma value), but also for the local dose differences normalized to the maximum value of the dose profiles (global gamma value). For all measurements, the high dose rate mode and the OptiVue 500 are used unless stated otherwise.

First, the accuracy of the dosimetric calibration model has been investigated for open fields using the EPID and the multidetector ionization chamber array. Wedge profiles in the wedge direction have been measured using a $6 \mathrm{MV}$ virtual wedge with a wedge angle of $60^{\circ}$ and a field size of $20 \mathrm{~cm} \times 20 \mathrm{~cm}$. Furthermore, a $10 \mathrm{MV}$ IMRT beam consisting of nine segments is delivered, and the dose is accumulated during all segments with the EPID and ionization chamber array. Dose comparisons are performed along a line through the central beam axis in cross-line and in-line directions. For both photon energies, on-axis and off-axis dose profiles obtained with the EPID and a scanning ionization chamber have been compared for a $10 \mathrm{~cm} \times 10 \mathrm{~cm}$ field shifted $5 \mathrm{~cm}$ off-axis and an irregular MLC field.

Second, the accuracy of the correction model for differences in energy spectrum has been determined. The thicknesses of a polystyrene slab phantom positioned with its midplane at the isocenter plane were 20.2 and $39.0 \mathrm{~cm}$. Dose profiles determined with the EPID and scanning ionization chamber have been compared for $6 \mathrm{MV}$ photon beams. The asymmetric $10 \mathrm{~cm} \times 10 \mathrm{~cm}$ field and the irregular MLC field have been used again for these verification measurements. Dose comparisons have also been performed behind an inhomogeneous phantom with a maximum thickness of $10.5 \mathrm{~cm}$ 
and an air cavity of $2.1 \mathrm{~cm}$ thickness, using a symmetric $20 \mathrm{~cm} \times 20 \mathrm{~cm}$ and the irregular MLC field.

\subsection{RESULTS}

\subsubsection{Long-term stability}

The long-term reproducibility of the response for $6 \mathrm{MV}$ photons was $0.7 \%, 0.5 \%$, and 0.5\% (1 SD) for the OptiVue 500, OptiVue 1000, and OptiVue 1000 ST EPID, respectively. For $10 \mathrm{MV}$ photons the reproducibility was $0.4 \%$ (1 SD) for the three EPIDs. Maximum differences were about 1.5\%. A gradual increase of EPID response from $-1.6 \%$ up to $+1.3 \%$ was observed for the OptiVue 500 EPID using 6 MV photons over a period of six months.

\subsubsection{Buildup}

Differences in relative EPID response of $2.5 \%$, with $\mathrm{Cu}$, and $6.2 \%$, without $\mathrm{Cu}$, were found for $6 \mathrm{MV}$ photons at an airgap of $25 \mathrm{~cm}$ compared to the relative response at the maximum airgap of $70 \mathrm{~cm}$. For an airgap of $50 \mathrm{~cm}$, the differences reduced to $0.6 \%$ and $1.7 \%$, respectively. For $10 \mathrm{MV}$ photons, differences of $1.4 \%$ with $\mathrm{Cu}$, and $10.6 \%$ without $\mathrm{Cu}$, were measured for an airgap of $25 \mathrm{~cm}$, while differences were again smaller for an airgap of $50 \mathrm{~cm}: 0.3 \%$ and $2.8 \%$, respectively.

The values of the critical frequency $f_{50}$ for $6 \mathrm{MV}$ photons with and without the Cu plate were both $0.38 \mathrm{lp} / \mathrm{mm}$, and no deterioration of clinical image quality was observed.

\subsubsection{Image lag and ghosting}

For each EPID, ghosting correction factors $G$ have been calculated for the high dose rate mode and for each photon beam energy. The mean values of the amplitudes $A$ and decay rates $r$ were $A_{1}=(216.54 \pm 0.22) \cdot 10^{-3}, A_{2}=0.490 \pm 0.027, A_{3}=0.0245 \pm 0.0095$, $r_{1}=13.58 \pm 0.35 \mathrm{~s}^{-1}, r_{2}=1.87 \pm 0.23 \mathrm{~s}^{-1}, r_{3}=0.077 \pm 0.045 \mathrm{~s}^{-1}$ (1 SD) for the $6 \mathrm{MV}$ photon beam. For the $10 \mathrm{MV}$ photon beam these values were $A_{1}=(196.42 \pm 4.45) \cdot 10^{-3}$, $A_{2}=0.019 \pm 0.027, A_{3}=0.022 \pm 0.012, r_{1}=15.90 \pm 4.18 \mathrm{~s}^{-1}, r_{2}=2.39 \pm 0.81 \mathrm{~s}^{-1}, r_{3}=0.070 \pm 0.014 \mathrm{~s}^{-1}$ (1 SD). Applying the calculated ghosting correction factors to the OptiVue 1000 measurements, the measured data could be matched within $0.2 \%$ accuracy for the high dose rate mode. For the low dose rate mode, the mean differences in response were $-0.6 \pm 0.4 \%$ and $-0.8 \pm 0.8 \%$ ( 1 SD) for the 6 and $10 \mathrm{MV}$ photon beams after correction, respectively. For $5 \mathrm{MUs}$, the decrease in response was $0.7 \%$ and $2.7 \%$, respectively, after application of the ghosting correction factors, which were determined for the high dose rate modes.

A $20 \mathrm{~cm} \times 20 \mathrm{~cm}$ treatment field was acquired immediately after exposing the OptiVue 1000 ST EPID using a $6 \mathrm{~cm} \times 6 \mathrm{~cm}$ field. Within the central $6 \mathrm{~cm} \times 6 \mathrm{~cm}$ region of the 20 $\mathrm{cm} \times 20 \mathrm{~cm}$ treatment field, grayscale values were increased compared to values of a portal image that was acquired after a long time interval without image acquisitions. 


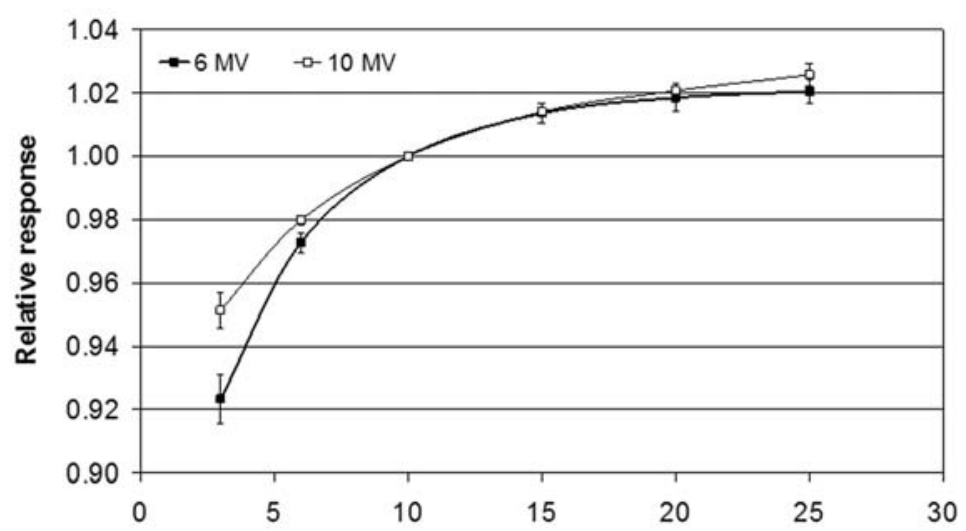

(a)

Field width $(\mathrm{cm})$

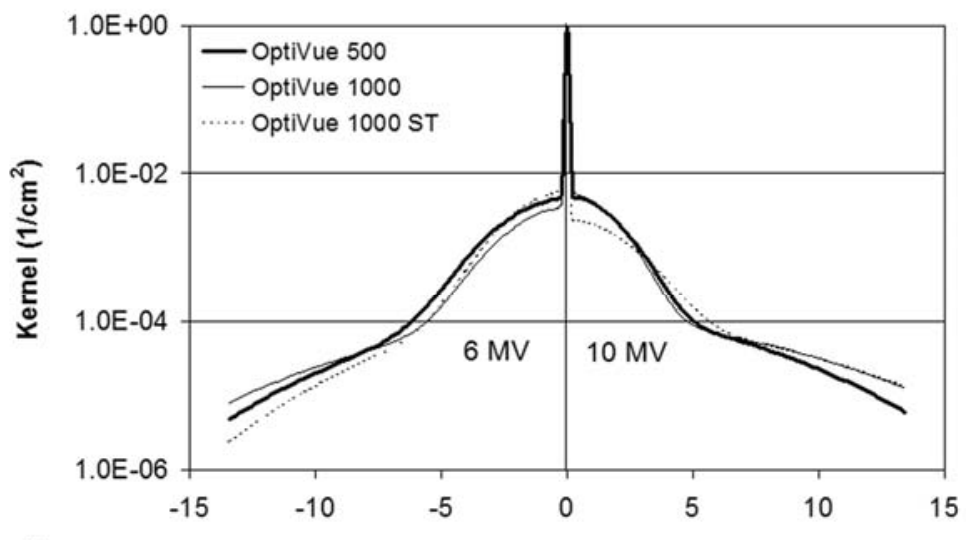

(b)

Off-axis position (cm)

Figure 5.3: (a) Measured relative EPID response (the ratio of the grayscale value $G_{\text {raw }}$ divided by the portal dose $D_{p}$ ) as a function of field width for 6 and $10 \mathrm{MV}$ photons with corresponding uncertainties (1 SD) normalized for a $10 \mathrm{~cm} \times 10 \mathrm{~cm}$ field. (b) Lateral extension of the field size dependence kernel $K_{F}$ for the OptiVue 500, OptiVue 1000, and OptiVue 1000 ST EPIDs for both photon energies. The kernels are normalized to 1 on the central axis.

The mean difference in grayscale values was $1.6 \pm 0.7 \%$ (1 SD) while the mean difference outside the $6 \mathrm{~cm} \times 6 \mathrm{~cm}$ region was $-0.1 \pm 0.6 \%$ (1SD).

\subsubsection{Field size dependence}

Figure 5.3(a) shows the mean relative response (the ratio of the grayscale value $G_{\text {raw }}$ divided by the portal dose $D_{p}$ ) of the three EPIDs for 6 and $10 \mathrm{MV}$ photon beams, normalized to the response for a $10 \mathrm{~cm} \times 10 \mathrm{~cm}$ field. If the field width decreases, the relative EPID response also decreases. The maximum under-response was $7.7 \pm 0.8 \%$ and $4.9 \pm 0.6 \%$ ( $1 \mathrm{SD}$ ) for the smallest field size of $3 \mathrm{~cm} \times 3 \mathrm{~cm}$ for the two photon 
energies, respectively. The maximum over-response was $2.1 \pm 0.4 \%$ and $2.6 \pm 0.4 \%$ ( 1 SD) for the largest field size of $24.2 \mathrm{~cm} \times 24.2 \mathrm{~cm}$. The field size dependence kernels $K_{F}$ were calculated for each EPID and for each photon beam, and are shown in Fig. 5.3(b). The best kernel fits were obtained if three Gaussian distributions were used to describe the field size dependence kernels: $N=3$ in Eq. (5.3). The mean values of the kernel coefficients $m$ and $\sigma$ were $m_{1}=0.497 \pm 0.061 \mathrm{~cm}^{-2}, m_{2}=0.0988 \pm 0.0056 \mathrm{~cm}^{-2}$, $m_{3}=0.00219 \pm 0.00030 \mathrm{~cm}^{-2}, \sigma_{1}=(5.36 \pm 0.23) \cdot 10^{-5} \mathrm{~cm}, \sigma_{2}=3.99 \pm 0.25 \mathrm{~cm}, \sigma_{3}=11.41 \pm 1.33$ $\mathrm{cm}(1 \mathrm{SD})$ for the $6 \mathrm{MV}$ photon beam. For the $10 \mathrm{MV}$ photon beam these values were $m_{1}=0.510 \pm 0.034 \mathrm{~cm}^{-2}, \quad m_{2}=0.0906 \pm 0.0085 \mathrm{~cm}^{-2}, \quad m_{3}=0.00208 \pm 0.00074 \mathrm{~cm}^{-2}$, $\sigma_{1}=(5.20 \pm 0.56) \cdot 10^{-5} \mathrm{~cm}, \sigma_{2}=3.50 \pm 0.59 \mathrm{~cm}, \sigma_{3}=13.35 \pm 1.47 \mathrm{~cm}$ (1 SD).

The mean corrected EPID response differences for the square field sizes were $0.0 \pm 0.2 \%$ and $-0.1 \pm 0.5 \%$ ( 1 SD) for 6 and $10 \mathrm{MV}$ photons, respectively. For the out-of-field region of the asymmetric field, the mean differences were $0.0 \pm 0.2 \%$ and $0.1 \pm 0.4 \%$ ( 1 SD), respectively.

\subsubsection{Absolute dose calibration}

The absolute portal dose value for $100 \mathrm{MUs}$ at the source-to-detector distance of 150 $\mathrm{cm}$ was 42.5 and $43.1 \mathrm{cGy}$ for the 6 and $10 \mathrm{MV}$ photon beams, respectively. For $6 \mathrm{MV}$ photons, the conversion factor $c_{F}$ was $5.47 \cdot 10^{4} \mathrm{cGy}^{-1}, 4.67 \cdot 10^{4} \mathrm{cGy}^{-1}$, and $3.76 \cdot 10^{4} \mathrm{cGy}^{-1}$ for the OptiVue 500/1000/1000 ST, respectively; for $10 \mathrm{MV}$ photons, values of $4.79 \cdot 10^{4}$

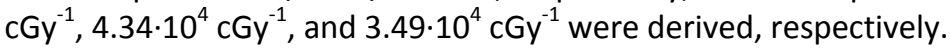

\subsubsection{Energy spectrum correction}

For all EPIDs, an under-response of the EPID was observed for increased off-axis positions and larger phantom thicknesses using the open field calibration (Sec. 5.2.2).

In Figs. 5.4(a) and 5.4(b), relative differences between the dose in water and EPID dose using Eq. (5.2) with $F=1$ are shown for a phantom thickness of 20.6 and $39.0 \mathrm{~cm}$, respectively, using a $6 \mathrm{MV}$ photon beam and a field size of $24.2 \mathrm{~cm} \times 24.2 \mathrm{~cm}$. Mean differences for the three EPIDs are on-axis $8.2 \pm 2.1 \%$ and $9.6 \pm 2.5 \%$ (1 SD) for the two phantom thicknesses, respectively. For a $10 \mathrm{~cm}$ off-axis position, these differences were larger: $14.0 \pm 2.4 \%$ and $16.2 \pm 3.6 \%$ (1 SD), respectively. Energy spectrum corrections $F_{1}$ were calculated for each EPID and for each photon beam energy to correct the differences between $G^{-1} \cdot G_{\text {raw }} \cdot B P$, and $G_{\text {ref }}$. The best fits were obtained by using a fourth degree polynomial: $N=5$ in Eq. (5.6). In Figs. 5.4(c) and 5.4(d), the energy spectrum correction factor $F_{1}$ is shown for the OptiVue 1000 ST EPID as a function of the off-axis position and the transmission $T$ for 6 and $10 \mathrm{MV}$ photon beams, respectively. The correction factor $F_{1}$ is much smaller for the $10 \mathrm{MV}$ than for the $6 \mathrm{MV}$ photon beam. By definition $F_{1}=1$ if no object is in the beam $(T=1)$. Systematic differences were observed in $F_{1}$ values between the OptiVue 1000 ST EPID and the other two EPIDs.

Relative differences between the dose in water and EPID dose using Eq. (5.2) with $F=F_{1}$ are shown in Figs. 5.5(a) and 5.5(b) for the same phantom thicknesses as applied for the data shown in Figs. 5.4(a) and 5.4(b), but now as a function of field area and on the 


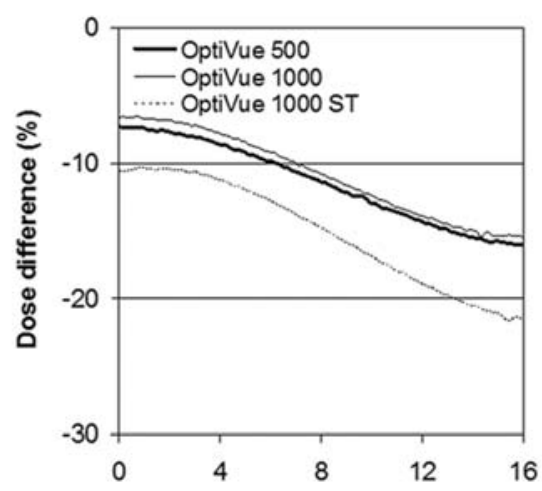

(a)

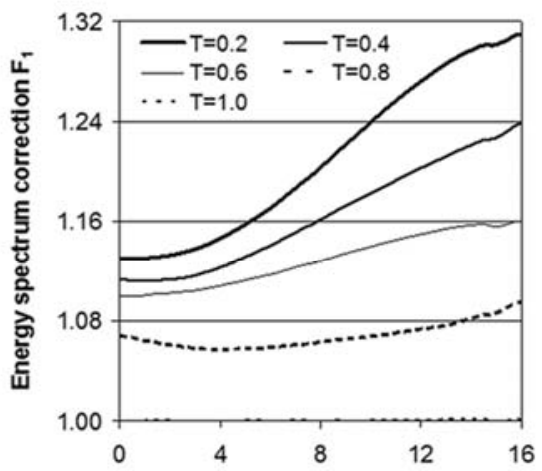

(c)
Off-axis position $(\mathrm{cm})$

Off-axis position $(\mathrm{cm})$

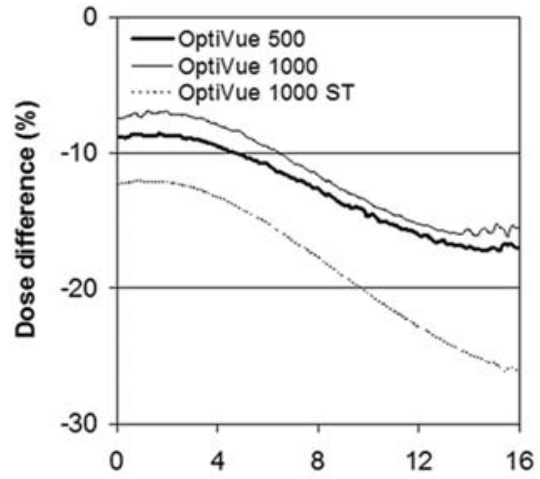

(b)

Off-axis position $(\mathrm{cm})$

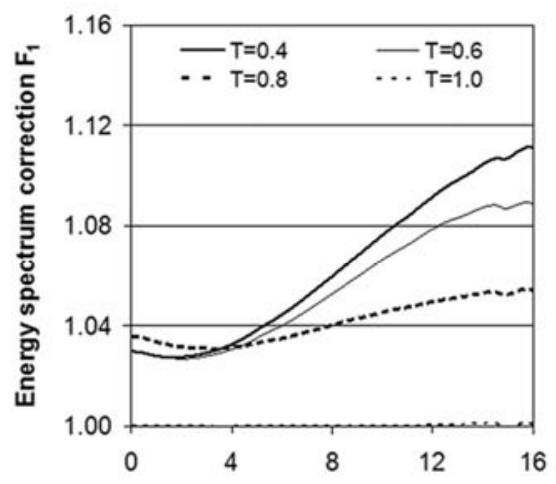

(d)

Off-axis position $(\mathrm{cm})$

Figure 5.4: Dose determined with the three Siemens OptiVue EPIDs using Eq. (5.2) with F=1 relative to the dose in water as a function of off-axis position behind a polystyrene slab phantom of (a) $20.6 \mathrm{~cm}$ and (b) $39.0 \mathrm{~cm}$ thickness positioned at the isocenter plane for $6 \mathrm{MV}$ photons. The energy spectrum correction $F_{1}$ for the OptiVue 1000 ST as a function of off-axis position is shown for different transmission values T for (c) $6 \mathrm{MV}$ photons and (d) $10 \mathrm{MV}$ photons.

central beam axis only. For smaller field areas, the under-response of the EPIDs increases up to a mean difference of $6.9 \pm 0.7 \%$ (1 SD) and $10.2 \pm 1.4 \%$ (1 SD) for the two phantom thicknesses, respectively. At small field areas, e.g., a field size of $3 \mathrm{~cm} \times 3 \mathrm{~cm}$, the under-response of the EPIDs reduces again. The kernel $K_{S}$ was fit with three Gaussian distributions and all fitted kernels were very similar. The mean values of the kernel coefficients $m$ and $\sigma$ were $m_{1}=0.111 \pm 0.026 \mathrm{~cm}^{-2}, m_{2}=0.12 \pm 0.19 \mathrm{~cm}^{-2}$, $m_{3}=0.26 \pm 0.20 \mathrm{~cm}^{-2}, \sigma_{1}=(5.30 \pm 0.31) \cdot 10^{-3} \mathrm{~cm}, \sigma_{2}=4.10 \pm 1.05 \mathrm{~cm}, \sigma_{3}=6.35 \pm 1.75 \mathrm{~cm}$ (1 SD), averaged for the three EPIDs and both photon beam energies. In Figs. 5.5(c) and 5.5(d), the scatter offsets $O_{s}$ are shown for the OptiVue 1000 ST EPID for 6 and 10 MV photon beams, respectively. For smaller field areas, the scatter offset increases while the dose differences shown in Figs. 5.5(a) and 5.5(b) decrease. No systematic differences could be observed in $F_{2}$ values when comparing the three EPIDs. 


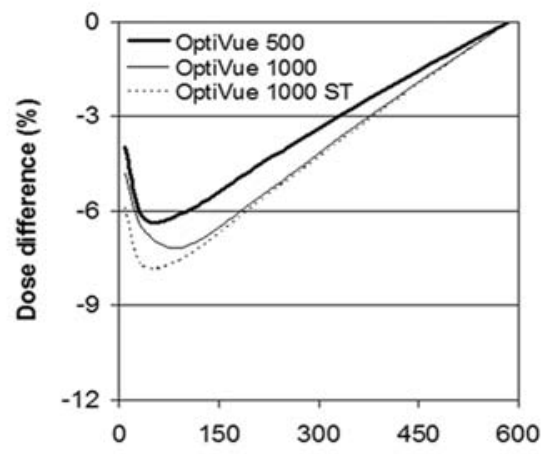

(a)

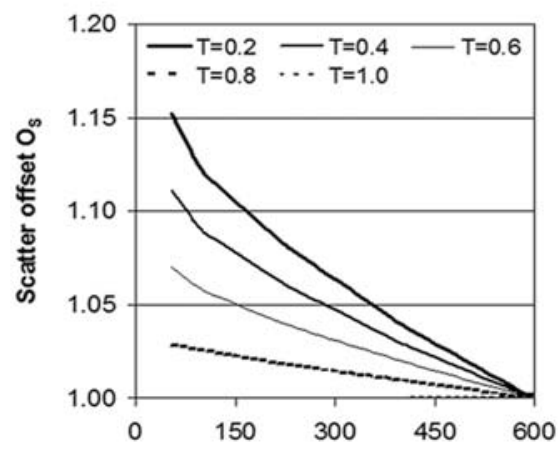

(c)
Field area $\left(\mathrm{cm}^{2}\right)$

Field area $\left(\mathrm{cm}^{2}\right)$

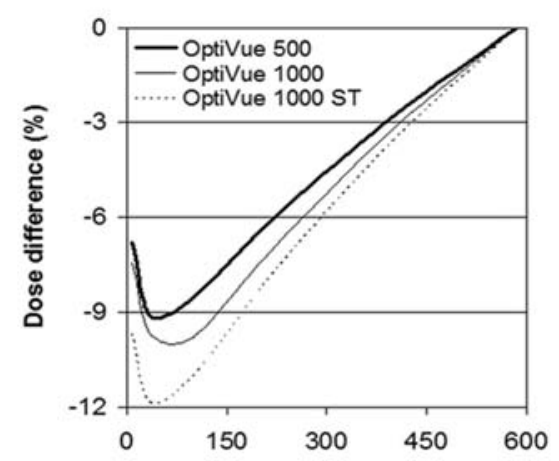

(b)

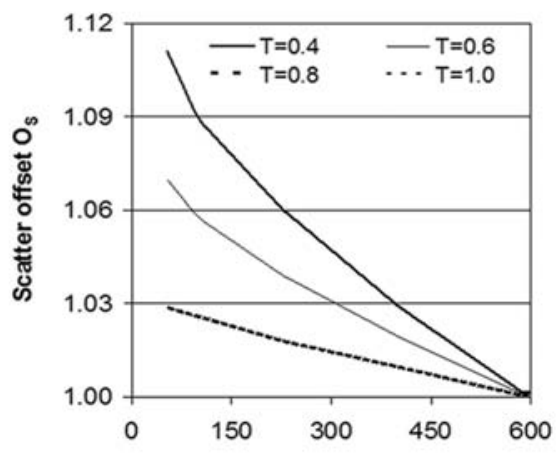

(d)

Field area $\left(\mathrm{cm}^{2}\right)$

Figure 5.5: Dose determined with the three Siemens OptiVue EPIDs using Eq. (5.2) with $F=F_{1}$ relative to the dose in water as a function of field area and on the central beam axis behind a polystyrene slab phantom of (a) $20.6 \mathrm{~cm}$ and (b) $39.0 \mathrm{~cm}$ thickness positioned at the isocenter plane for $6 \mathrm{MV}$ photons. The scatter offset $O_{S}$ for the OptiVue 1000 ST as a function of field area is shown for different transmission values $T$ for (c) $6 \mathrm{MV}$ photons and (d) $10 \mathrm{MV}$ photons; the transmission values are corrected by the energy spectrum correction $F_{1}$.

\subsubsection{Verification measurements}

In Fig. 5.6(a), $6 \mathrm{MV}$ virtual wedge profiles along a line through the central beam axis are shown for the absolute dose in water and EPID measurements (left $y$ axis). Local gamma values have been calculated (right $y$ axis) and were on average $0.6 \pm 0.3$ (1 SD) while about $10.3 \%$ of all local gamma values were larger than 1 . For wedge profiles at an off-axis position of $10 \mathrm{~cm}$ in the EPID plane (not shown), the mean local gamma value was $0.6 \pm 0.4$ ( $1 \mathrm{SD}$ ) with $17.3 \%$ of the local gamma values larger than 1 . In Fig. $5.6(b)$, in-line dose profiles are shown through the central beam axis of a 10 MV IMRT beam. Only $2.2 \%$ of the local gamma values were larger than 1 , and the mean local 

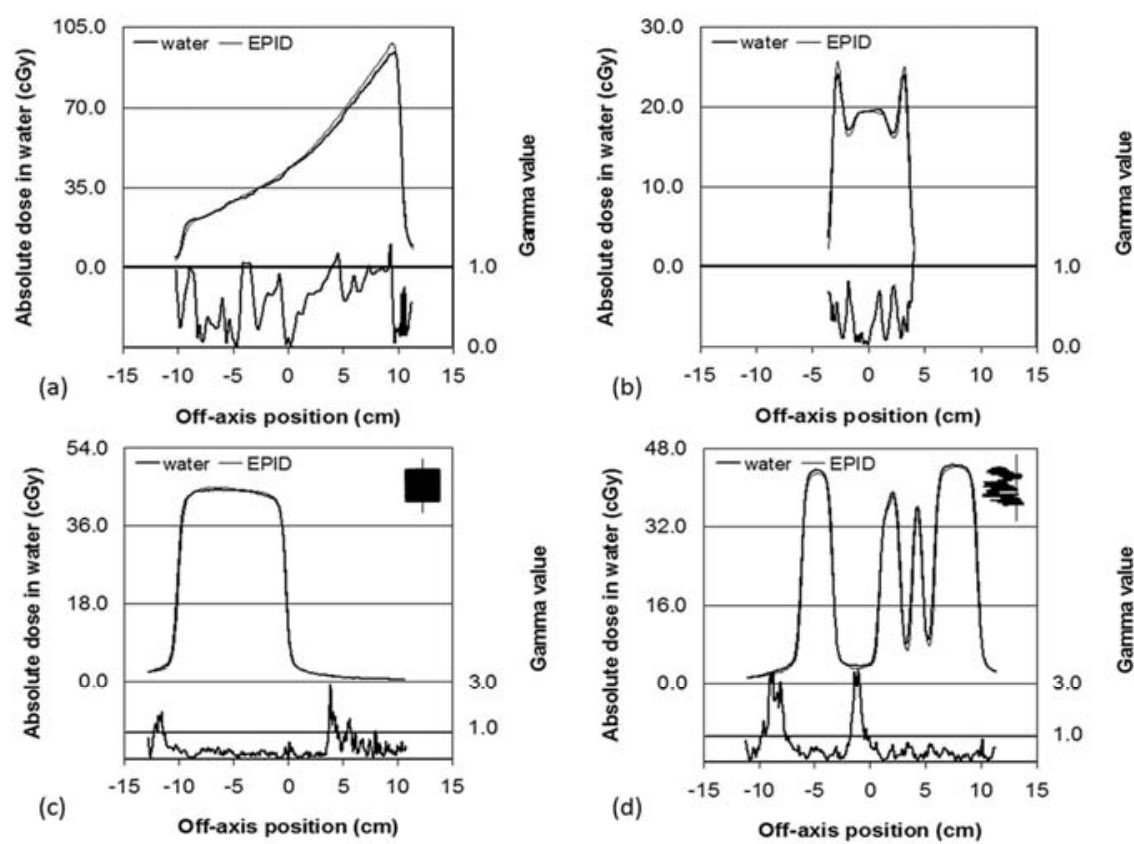

Figure 5.6: Absolute dose profiles (left y axis) measured in water and by the OptiVue 500 EPID for (a) a $6 \mathrm{MV}$ photon beam with a virtual wedge of $60^{\circ}$, (b) a 9 segment IMRT field using $10 \mathrm{MV}$ photons, (c) a $6 \mathrm{MV} 10 \mathrm{~cm} \times 10 \mathrm{~cm}$ field shifted $5 \mathrm{~cm}$ off-axis in two directions, and (d) an irregular 6 MV MLC field. A small representation of the latter two treatment fields and dose profile locations can be seen in the corner of $(c)$ and $(d)$. Only treatment fields without a phantom in the beam are used, and in-line profiles are shown. Local gamma values are calculated for each pair of dose profiles and indicated on the right $y$ axis.

gamma value was $0.4 \pm 0.2$ ( $1 \mathrm{SD}$ ). For the cross-line profile (not shown), $5.1 \%$ of the local gamma values were larger than 1 with a mean value of $0.4 \pm 0.3$ (1 SD). In Figs. $5.6(\mathrm{c})$ and 5.6(d), dose profiles for an asymmetric $10 \mathrm{~cm} \times 10 \mathrm{~cm}$ field and an irregular MLC field are shown. All in-field local gamma values were smaller than 1 with a mean local gamma value of $0.3 \pm 0.1$ ( $1 \mathrm{SD}$ ) for the two open fields, respectively. Out-of-field, $44.3 \%$ and $31.0 \%$ of all local gamma values were larger than 1 ; mean local gamma values were $1.4 \pm 1.4$ and $1.0 \pm 1.0(1 \mathrm{SD})$, respectively and corresponding mean relative dose differences were $-8.2 \pm 17.9 \%$ and $10.0 \pm 7.6 \%$ ( $1 \mathrm{SD}$ ), respectively. In case of a global gamma evaluation, all out-of-field global gamma values were smaller than 1 and the mean global gamma values were $0.1 \pm 0.1$ and $0.2 \pm 0.1$ ( $1 \mathrm{SD}$ ) for the two open fields, respectively.

In Figs. 5.7(a) and 5.7(b), absolute dose profiles are shown for the irregular MLC field and the polystyrene slab phantom of $39.0 \mathrm{~cm}$ with and without the energy spectrum correction $F_{1}$ applied to the out-of-field regions, respectively. In-field, local gamma evaluations gave the same result in both cases. Less than $0.7 \%$ of all gamma values was larger than 1 , and the mean gamma value was $0.4 \pm 0.2$ (1 SD). Out-of-field, agreement between the dose profiles in water and at the EPID was much better if $F_{1}$ was only 

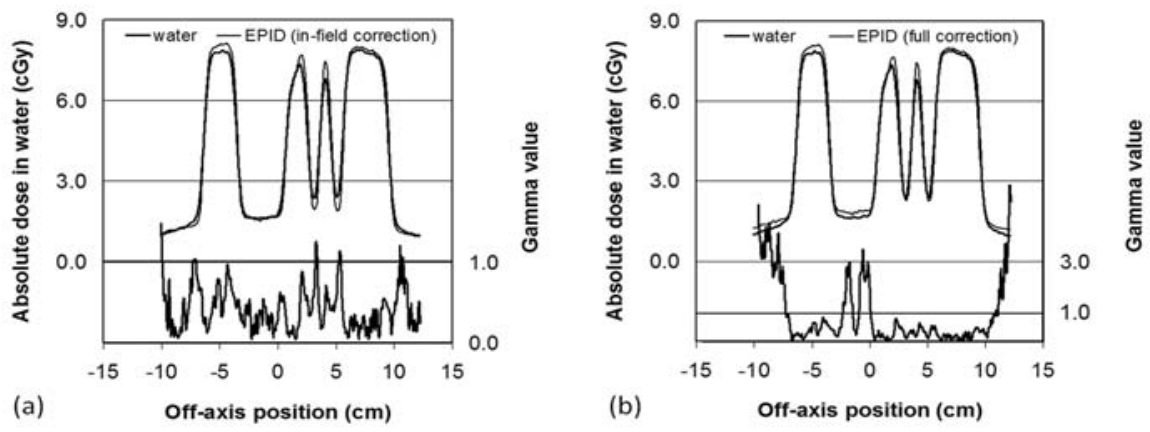

Figure 5.7: Absolute dose profiles measured in water and by the OptiVue 500 EPID for the irregular 6 MV MLC field depicted in Fig. $5.6($ d). A $39.0 \mathrm{~cm}$ thick polystyrene slab phantom was positioned at the isocenter plane, and the energy spectrum correction $F_{1}$ was used (a) for the entire image excluding the out-of-field regions and (b) for the entire image. Dose profiles are indicated on the left $y$ axis and corresponding local $\gamma$ values on the right $y$ axis.
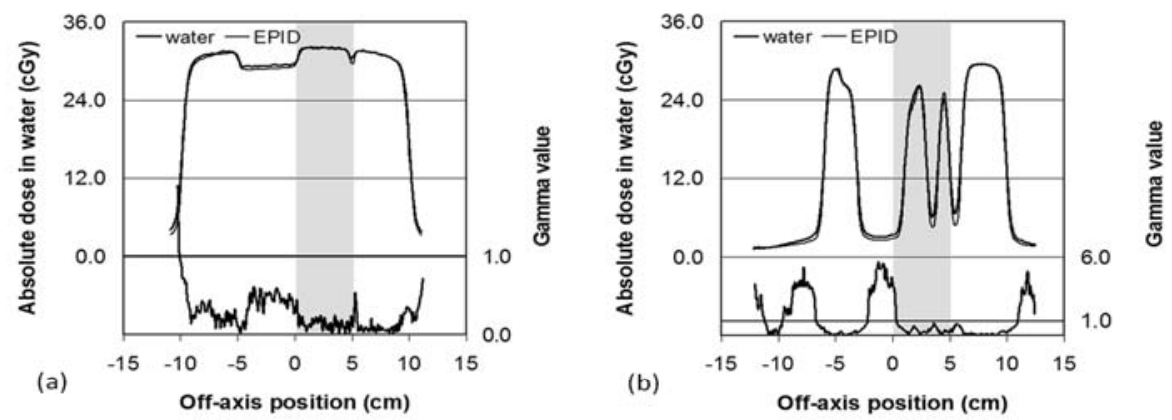

Figure 5.8: Absolute dose profiles measured behind an inhomogeneous phantom in water and by the OptiVue 500 EPID (left $y$ axis) and $\gamma$ values (right y axis). The phantom consisted of a stack of polystyrene slabs with a maximum thickness of $10.5 \mathrm{~cm}$ and an air cavity of $2.1 \mathrm{~cm}$ thickness at a position marked by the shaded region. The dose profiles in (a) and (b) are measured for a $20 \mathrm{~cm} x$ $20 \mathrm{~cm}$ field and for the irregular MLC depicted in Fig. 5.6(d), using 6 MV photons.

applied in-field. Comparing Figs. 5.7(a) and 5.7(b), 25.0\% and $66.3 \%$ of all local gamma values were larger than 1 , respectively. In case of a global gamma evaluation, these percentages were $4.9 \%$ and $14.7 \%$, respectively. Mean local gamma values were $1.3 \pm 1.9$ and $2.9 \pm 2.6(1 \mathrm{SD})$ and mean global gamma values were $0.4 \pm 0.3$ and $0.7 \pm 0.4$ (1 SD), respectively. Corresponding mean relative dose differences were $1.1 \pm 15.4 \%$ and $-15.2 \pm 13.7 \%$. For the asymmetric $10 \mathrm{~cm} \times 10 \mathrm{~cm}$ field and the same phantom thickness, the mean relative dose difference was $0.4 \pm 12.9 \%$ when $F_{1}$ was only applied in-field.

For other polystyrene slab phantom thicknesses in combination with the asymmetric $10 \mathrm{~cm} \times 10 \mathrm{~cm}$ field and the irregular MLC field, dose profile comparisons were similar to the latter results. In-field, all measurements fulfilled the local and global gamma 
criteria of $3 \%$ and $3 \mathrm{~mm}$. In the out-of-field regions, a large variation in relative dose differences could be found and for phantom thicknesses smaller than $39.0 \mathrm{~cm}$, an under-response of the EPID was observed.

In case of an inhomogeneous phantom with air cavities, local gamma values larger than 1 were only observed in out-of-field regions close to density interfaces. Out-of-field, mean local and global gamma values were $0.9 \pm 0.8$ and $0.5 \pm 0.2$ (1 SD), respectively, for a $20 \mathrm{~cm} \times 20 \mathrm{~cm}$ field; for the irregular MLC field, these values were $2.2 \pm 1.7$ and $0.4 \pm 0.2$ ( $1 \mathrm{SD}$ ), respectively. Mean relative dose differences were $17.8 \pm 8.0 \%$ and $14.0 \pm 11.8 \%$ ( $1 \mathrm{SD}$ ). In Figs. 5.8(a) and 5.8(b), the absolute dose profiles are shown for both treatment fields.

\subsection{DISCUSSION}

\subsubsection{Long-term stability}

On average, the variation in long-term response was small and amounts to about $0.5 \%$ (1 SD) for all three EPIDs and both photon energies. Louwe et al. ${ }^{12}$ described the long-term stability of four Elekta iView GT EPIDs and found a similar variation after applying a dark current correction. The dark current correction removes temperature fluctuations and the influence of a small, irreversible increase of the dark-field current probably caused by mild radiation damage to the $a-S i$ array. Menon and Sloboda ${ }^{21}$ reported a drift in response for the Varian aS500 EPID of up to 4\%, depending on the beam quality, over a period of 5 months, which has also been observed in our work for the OptiVue 500 EPID. This drift in response and, hence, EPID dose can be corrected by measuring the conversion factor $c_{F}$ more frequently, e.g., once a month.

\subsubsection{Image lag and ghosting}

McDermott et al. $^{22}$ compared the signal-to-monitor unit ratio for three different brands of EPID systems including the Siemens OptiVue 500 and OptiVue 1000 applied in this study. It was found that all EPIDs exhibited a relative under-response for beams delivering few monitor units, up to $10 \%$, which can be explained by ghosting effects due to charge trapping. Furthermore, a dose rate dependence of the EPID response was reported that can be attributed to changes in dose per subframe. ${ }^{23}$ In our work, ghosting correction factors are calculated for high dose rate measurements only and applied to measurements with both dose rate modes. The values of the ghosting correction factors can be compared to values in McDermott et al. ${ }^{11}$ and have the same magnitude. In case of virtual wedge treatments, the dose rate changes over the wedge profile, which could result in less accurate EPID response corrections for image lag. The differences in wedge profile measured with the EPID and the multidetector ionization chamber array are located at the high dose part of the wedge profile. An incomplete image lag correction could not cause these differences, because the maximum under-response is only $0.7 \%$ for $6 \mathrm{MV}$ photons. For the virtual wedge and IMRT fields, the amount of radiation at the EPID changes continuously during treatment delivery, 
which means that a ghosting correction factor should be position dependent. The verification measurements did not show dose differences that were caused by the fact that only one specific value for the ghosting correction factor was used.

Winkler et al. $^{23}$ found a maximum increase in relative response of about $5 \%$ for the Elekta iView GT EPID due to ghosting. In our work only $1.6 \pm 0.7 \%$ (1 SD) increase was found under the same measurement conditions. These differences in ghosting effect may be caused by slightly different acquisition characteristics of the Siemens EPIDs compared to the Elekta EPIDs.

\subsubsection{Field size dependence and beam profile correction}

Warkentin et al. $^{18}$ modeled the field size dependence of the aS500 EPID response by three separate kernels per beam energy using measurements and MC simulations. The first kernel accounts for energy deposition in the phosphor screen, and the second kernel describes the optical photon spread from the screen to the photodiode layer. Both kernels can be deconvolved with a portal image to obtain a primary fluence image. Convolution with the third kernel, an energy deposition kernel in water, results in a full-scatter PDI. A similar convolution approach was chosen by Chen et al. ${ }^{20}$ but now empirical kernels were utilized. In our work, one field size dependence kernel $K_{F}$ is calculated per beam energy based on a limited set of measurements only and by using three Gaussians. Special attention was paid to the out-of-field region to fit the broader Gaussian of the kernel. Because the EPIDs have a larger field size dependence compared to the dose in water at a depth of $5 \mathrm{~cm}$, a deconvolution of $K_{F}$ with the grayscale value distribution $G_{\text {raw }}$ is needed. EPID response differences were smaller than $0.5 \%$ (1 SD) in-field and out-of-field using our kernel optimization procedure.

Based on the verification measurements without an object in the beam, it can be concluded that the field size dependence kernel is invariant with off-axis position because infield gamma values are almost never larger than 1, even not for an IMRT field with very asymmetric segment field shapes and for the irregular MLC field. The few gamma values larger than 1 occurred mainly in steep dose gradient regions and could be explained by the use of the $0.6 \mathrm{~cm}$ diameter ionization chamber for the measurements in water.

\subsubsection{Buildup and energy spectrum correction}

McDermott et al. ${ }^{11}$ found that copper is a better buildup material than polystyrene because the over-response was smaller for equal density thickness, and less volume was occupied on top of the EPID. For photon energies varying between 6 and $18 \mathrm{MV}$, they found that an additional $\mathrm{Cu}$ buildup layer of $2.5 \mathrm{~mm}$ decreased the EPID over-response to within $4 \%$ for a minimum airgap of $20 \mathrm{~cm}$ between a polystyrene slab phantom and EPID compared to the response at an airgap of $60 \mathrm{~cm}$. Our findings are in agreement with these results. The $f_{50}$ values for $6 \mathrm{MV}$ photons did not decrease by the $3 \mathrm{~mm}$ additional $\mathrm{Cu}$ plate on the EPID, which corresponds to the findings of Partridge et $a l^{27}$ These authors showed that there is no significant effect on the spatial resolution of indirect-detection panels by adding 3 to $4 \mathrm{~mm} \mathrm{Cu}$. McDermott et al. ${ }^{11}$ 
reported that additional $\mathrm{Cu}$ caused a slight reduction in image quality by qualitative checks of clinical images which can be neglected for patient treatment set-up verification.

Although the $3 \mathrm{~mm} \mathrm{Cu}$ layer reduces the over-response of the EPID by filtering low energy scattered photons from a phantom or patient, still a large residual variation in EPID response as a function of photon energy remains. Kirkby et al. ${ }^{16}$ investigated the use of copper as buildup and described better results for an elevated configuration of $15 \mathrm{~cm}$ above the $a$-Si array than for a contact configuration. For a copper thickness of about $7 \mathrm{~mm}$, the maximum EPID response difference simulated by MC calculations was smaller than $4 \%$ for the elevated configuration while the difference was much larger with the copper plate in the contact configuration. Because more space is occupied by the elevated configuration, this would decrease the clearance between patient and EPID, and hence the number of treatment fields that can be imaged in clinical practice. Also, a Cu thickness larger than $3 \mathrm{~mm}$ increases the weight of the EPID to a technically undesirable situation. Therefore, an energy spectrum correction is a better approach to correct the residual differences in EPID response, which could be as large as $17.2 \pm 0.7 \%(1 \mathrm{SD})$ as observed in this study for a phantom thickness of $39.0 \mathrm{~cm}$ at an off-axis position of $10 \mathrm{~cm}$ isocentrically using $6 \mathrm{MV}$ photons.

The energy spectrum correction described in our work is based on measured transmission distributions and field area, and hence no input of beam characteristics is needed. Chen et al. $^{20}$ proposed a model based on the EPID signal itself, which implies that the number of monitor units is an input parameter of the model to determine energy spectrum correction factors. Especially in the case of transit dosimetry for wedged or IMRT fields, this approach may introduce errors in the conversion procedure from grayscale EPID images to full-scatter PDIs because the corresponding energy fluence should be exactly the same as delivered by the treatment machine. For the application of our energy spectrum correction model, it is necessary that EPID images be acquired during a dummy session without a patient in the beam before the actual treatment starts. Although this takes some extra time, these images can be converted to PDIs and used as a pre-treatment verification of the delivery by the treatment machine.

Verification measurements show that the energy spectrum correction factor $F$ gives very good results for the in-field region of different treatment fields fulfilling gamma criteria of $3 \%$ and $3 \mathrm{~mm}$ for more than $99 \%$ of all gamma values in this region. In the out-of-field regions, mean relative dose differences were rather small for a homogeneous phantom thickness of $39.0 \mathrm{~cm}$ (within 1.1\%) indicating that the energy spectrum correction model $F$ works very well. Out-of-field, changes in energy spectrum are predominantly due to patient scatter and are corrected by using the scatter kernel $K_{S}$ applied to a constant scatter offset $O_{S}$ within the treatment field. The energy response correction $F_{1}$ is equal to 1 at these out-of-field regions. This means that the photon energies behind the collimating jaws and the MLC are outside the range where the EPID response of $a$-Si EPIDs will be overestimated due to beam hardening. For other homogeneous phantom thicknesses, the EPID response was underestimated out-of-field, which can be explained by the fact that the scatter kernel $K_{S}$ is derived at 
the phantom thickness of $39.0 \mathrm{~cm}$ only. For a smaller phantom thickness, the contribution of in-field patient scatter to the out-of-field regions should be smaller, which means that the contribution of kernel $K_{S}$ to $F_{2}$ out-of-field should be larger. This can be modeled by determining the parameters of $K_{S}$ as a function of the mean value of $F_{1} \cdot T$ again. The variation in relative dose differences could be up to $20 \%$ (1 SD), which can be explained by the noisy signals for the dose in water and EPID measurements.

Our correction method is based on transmission values measured at the in-field region where the major part of patient scatter originates. The energy spectrum correction $F_{2}$ uses a constant scatter offset, which is a function of the mean transmission within a treatment field. In case of an inhomogeneous phantom/patient, the transmission value measured just inside the field edges could differ from the mean transmission value and hence dose differences could be introduced in the out-of-field regions. For the inhomogeneous phantom used in this work, changes in transmission were not very large, and the mean relative dose differences could again be explained by the energy independent kernel $K_{S}$ that was used. Furthermore, mean transmission and irradiated field area were determined by segmentation of field edges in an EPID image. In clinical practice, it is more accurate to use segmentation of the patient contours using a transmission distribution (e.g., for tangential breast fields), because patient scatter and hence $F_{2}$ are related to the irradiated patient volume and not the radiation volume.

\subsubsection{EPID variability}

The EPID response as a function of field size and the ghosting correction factors were very similar for the OptiVue 500/1000/1000 ST $a$-Si EPIDs. Good agreement was found between the measured energy spectrum corrections for the OptiVue 500 and 1000 EPIDs with differences smaller than 1\% (1 SD) for each position in the EPID plane. The energy spectrum response correction for the OptiVue 1000 ST EPID was different, which can be explained by the larger $a$-Si layer thickness and hence slightly different radiation interaction characteristics. These findings allow the use of one data set per photon beam energy for the OptiVue 500 and OptiVue 1000 to describe image lag, field size dependence, and energy spectrum correction in clinical practice. Because all linear accelerators in this work were dosimetrically matched, the beam profile correction $B P$ is only dependent on photon beam energy and not on EPID type.

\subsubsection{SDD dependency of the model}

In our study, only one source-to-detector distance of $150 \mathrm{~cm}$ was used; for other distances, additional measurements have to be performed to assess the field size dependence kernel $K_{F}$ as a function of SDD as described by Van Esch et al. ${ }^{10}$ The beam profile correction $B P$ and the parameters in our energy spectrum correction $F$ can approximately be rescaled to other source-to-detector distances using the inverse square law, avoiding new measurements. However, the patient scatter contribution to the EPID will increase significantly for smaller source-to-detector distances, ${ }^{19}$ probably decreasing the accuracy of our energy spectrum correction model. 


\subsubsection{Global applicability of the model}

The dosimetric calibration model described in our work can be applied to other indirect $a$-Si EPIDs than the Siemens OptiVue EPIDs relatively easily because only a limited set of measurements is necessary to determine the model parameters. No MC simulations, which require machine and EPID geometry or energy spectrum, have to be done. Furthermore, the dose characteristics of the Siemens OptiVue EPIDs are similar to the dose characteristics of the Varian aS500 and Elekta iView GT EPID considering reported ghosting effects, field size dependence, and energy spectrum dependence.

\subsection{CONCLUSIONS}

We have developed a global dosimetric calibration procedure for indirect $a$-Si EPIDs to convert grayscale EPID images into full-scatter portal dose images to be used for transit dosimetry applications. For this purpose, a model has been investigated that corrects for the photon energy dependence of the EPID response based on measured transmission distributions, without the need of input of beam characteristics. Long-term stability, buildup, ghosting effects, field size dependence, and sensitivity of the EPID were determined for three new commercially available $a$-Si EPIDs in order to take into account during the dosimetric calibration process. PDIs were derived for different treatment fields such as virtual wedge and IMRT fields, with various (in)homogeneous phantoms in the field, to determine the accuracy and applicability of the energy spectrum correction model. Gamma evaluation criteria of $3 \%$ and $3 \mathrm{~mm}$ were satisfied for all measurements across the entire EPID plane in-field. Larger dose differences were found out-of-field but could be minimized by deriving an energy dependent scatter kernel based on transmission values. Using our energy spectrum correction model, highly accurate portal dose images can be obtained which are required for $2 \mathrm{D}$ transit dosimetry applications ${ }^{28}$ and $3 \mathrm{D}$ dose reconstruction. ${ }^{29}$ 


\section{REFERENCES}

1 J.J. Sonke, L.S. Ploeger, B. Brand, M.H.P. Smitsmans, and M.B. van Herk, "Leaf trajectory verification during dynamic intensity modulated radiotherapy using an amorphous silicon flat panel imager," Med. Phys. 31, 389-395 (2004).

2 M.G. Herman, J.J. Kruse, and C.R. Hagness, "Guide to clinical use of electronic portal imaging," J. Appl. Clin. Med. Phys. 1, 38-57 (2000).

3 S.C. Vieira, M.L.P. Dirkx, K.L. Pasma, and B.J.M. Heijmen, "Fast and accurate leaf verification for dynamic multileaf collimation using an electronic portal imaging device," Med. Phys. 29, 2034-2040 (2002).

4 S.M.J.J.G. Nijsten, A.W.H. Minken, P. Lambin, and I.A.D. Bruinvis, "Verification of treatment parameter transfer by means of electronic portal dosimetry," Med. Phys. 31, 341-347 (2004).

5 A. Van Esch, B. Vanstraelen, J. Verstraete, G. Kutcher, and D. Huyskens, "Pretreatment dosimetric verification by means of a liquid-filled electronic portal imaging device during dynamic delivery of intensity modulated treatment fields," Radiother. Oncol. 60, 181-190 (2001).

6 K.L. Pasma, M. Kroonwijk, S. Quint, A.G. Visser, and B.J.M. Heijmen, "Transit dosimetry with an electronic portal imaging device (EPID) for 115 prostate cancer patients," Int. J. Radiat. Oncol., Biol., Phys. 45, 1297-1303 (1999).

7 S.M.J.J.G. Nijsten, B.J. Mijnheer, A.L.A.J. Dekker, P. Lambin, and A.W.H. Minken, "Routine individualised patient dosimetry using electronic portal imaging devices," Radiother. Oncol. 83, 65-75 (2007).

8 P.B. Greer and C.C. Popescu, "Dosimetric properties of an amorphous silicon electronic portal imaging device for verification of dynamic intensity modulated radiation therapy," Med. Phys. 30, 1618-1627 (2003).

9 E.E. Grein, R. Lee, and K. Luchka, "An investigation of a new amorphous silicon electronic portal imaging device for transit dosimetry," Med. Phys. 29, 2262-2268 (2002).

10 A. Van Esch, T. Depuydt, and D.P. Huyskens, "The use of an aSi-based EPID for routine absolute dosimetric pretreatment verification of dynamic IMRT fields," Radiother. Oncol. 71, 223-234 (2004).

11 L.N. McDermott, R.J.W. Louwe, J.J. Sonke, M.B. van Herk, and B.J. Mijnheer, "Dose-response and ghosting effects of an amorphous silicon electronic portal imaging device," Med. Phys. 31, 285-295 (2004).

12 R.J.W. Louwe, L.N. McDermott, J.J. Sonke, R. Tielenburg, M. Wendling, M.B. van Herk, and B.J. Mijnheer, "The long-term stability of amorphous silicon flat panel imaging devices for dosimetry purposes," Med. Phys. 31, 2989-2995 (2004).

13 C. Yeboah and S. Pistorius, "Monte Carlo studies of the exit photon spectra and dose to a metal/phosphor portal imaging screen," Med. Phys. 27, 330-339 (2000).

14 P.B. Greer, "Correction of pixel sensitivity variation and off-axis response for amorphous silicon EPID dosimetry," Med. Phys. 32, 3558-3568 (2005).

15 B.M.C. McCurdy, K. Luchka, and S. Pistorius, "Dosimetric investigation and portal dose image prediction using an amorphous silicon electronic portal imaging device," Med. Phys. 28, 911-924 (2001).

16 C. Kirkby and R. Sloboda, "Consequences of the spectral response of an $a$-Si EPID and implications for dosimetric calibration," Med. Phys. 32, 2649-2658 (2005).

17 W. Li, J.V. Siebers, and J.A. Moore, "Using fluence separation to account for energy spectra dependence in computing dosimetric a-Si EPID images for IMRT fields," Med. Phys. 33, 4468-4480 (2006). 
18 B. Warkentin, S. Steciw, S. Rathee, and B.G. Fallone, "Dosimetric IMRT verification with a flat-panel EPID," Med. Phys. 30, 3143-3155 (2003).

19 W.J.C. van Elmpt, S.M.J.J.G. Nijsten, B.J. Mijnheer, and A.W.H. Minken, "Experimental verification of a portal dose prediction model," Med. Phys. 32, 2805-2818 (2005).

20 J. Chen, C.F. Chuang, O. Morin, M. Aubin, and J. Pouliot, "Calibration of an amorphous-silicon flat panel portal imager for exit-beam dosimetry," Med. Phys. 33, 584-594 (2006).

21 G.V. Menon and R.S. Sloboda, "Quality assurance measurements of $a$-Si EPID performance," Med. Dosim. 29, 11-17 (2004).

22 L.N. McDermott, S.M.J.J.G. Nijsten, J.J. Sonke, M. Partridge, M.B. van Herk, and B.J. Mijnheer, "Comparison of ghosting effects for three commercial $a$-Si EPIDs," Med. Phys. 33, 2448-2451 (2006).

23 P. Winkler, A. Hefner, and D. Georg, "Dose-response characteristics of an amorphous silicon EPID," Med. Phys. 32, 3095-3105 (2005).

24 R. Boellaard, M.B. van Herk, and B.J. Mijnheer, "A convolution model to convert transmission dose images to exit dose distributions," Med. Phys. 24, 189-199 (1997).

25 D.A. Low, W.B. Harms, S. Mutic, and J.A. Purdy, "A technique for the quantitative evaluation of dose distributions," Med. Phys. 25, 656-661 (1998).

26 D.A. Low and J.F. Dempsey, "Evaluation of the gamma dose distribution comparison method," Med. Phys. 30, 2455-2464 (2003).

27 M. Partridge, B.M. Hesse, and L. Müller, "A performance comparison of direct- and indirect-detection flat-panel imagers," Nucl. Instrum. Methods Phys. Res. A 484, 351-363 (2002).

28 M. Kroonwijk, K.L. Pasma, S. Quint, P.C.M. Koper, A.G. Visser, and B.J.M. Heijmen, "In vivo dosimetry for prostate cancer patients using an electronic portal imaging device (EPID); Demonstration of internal organ motion," Radiother. Oncol. 49, 125-132 (1998).

29 W.J.C. van Elmpt, S.M.J.J.G. Nijsten, R.F.H. Schiffeleers, A.L.A.J. Dekker, B.J. Mijnheer, P. Lambin, and A.W.H. Minken, "A Monte Carlo based three-dimensional dose reconstruction method derived from portal dose images," Med. Phys. 33, 2426-2434 (2006). 



\section{CHAPTER}

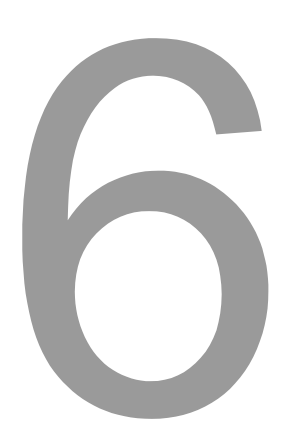

\section{Comparison of ghosting effects for three commercial $a$-Si EPIDs}

Med. Phys. 33(7), 2448-2451 (2006)

L.N. McDermott

S.M.J.J.G. Nijsten J.J. Sonke

M. Partridge M.B. van Herk

B.J. Mijnheer 



\section{ABSTRACT}

Many studies have reported dosimetric characteristics of amorphous silicon electronic portal imaging devices (EPIDs). Some studies ascribed a non-linear signal to gain ghosting and image lag. Other reports, however, state the effect is negligible. This study compares the signal-to-monitor unit (MU) ratio for three different brands of EPID systems. The signal was measured for a wide range of monitor units (5-1000), dose-rates, and beam energies. All EPIDs exhibited a relative under-response for beams of few MUs; giving 4 to $10 \%$ lower signal-to-MU ratios relative to that of 1000 MUs. This under-response is consistent with ghosting effects due to charge trapping. 


\subsection{INTRODUCTION}

Dosimetry with portal imagers is becoming increasingly popular, offering the potential for multi-dimensional dose verification. There are currently three brands of amorphous silicon electronic portal imaging devices ( $a$-Si EPIDs) commercially available: Elekta iView GT (Elekta, Crawley, United Kingdom), Varian aS500/1000 (Varian Medical Systems, Palo Alto, California), and Siemens OptiVue 500/1000 (Siemens Medical Solution, Concord, California).

Before using such a device for dose verification, it is necessary to first determine its dosimetric characteristics. Signal-to-dose ratios have been measured for these types of detectors, and found to be non-constant. ${ }^{1,2}$ A lower signal-to-MU ratio was reported for relatively short irradiation times, up to $10 \%$ lower than that of longer irradiation times for the Elekta EPID. The source of the deviation was attributed to image lag and gain ghosting effects. "Image lag" is due to charge trapped in the photodiode bulk modulus or at the surface. Trapped charge read out in subsequent frames results in an offset of the EPID signal. "Gain ghosting" refers to the change in gain, or pixel sensitivity, due to the trapped charge, which alters the electric field strength in the bulk or surface of the photodiode layer. The extent of both effects (image lag and gain ghosting) will depend on both the panel design and the exposure time. Trapping in the bulk layers effectively involves the "direct capture of charge at defect energy levels in the gap and is followed by the slow release over a broad range of time constants." ${ }^{3}$ In particular, the design and manufacture of the diode layer will influence the density of trapping states, and hence influence the way charge is trapped at the diode level. Various reports have investigated image lag and gain ghosting properties of indirect flat panel detectors in further detail. ${ }^{3-6}$

When using the EPID as a dosimeter, both image lag and gain ghosting effects combine to influence the dose per frame read out by the detector. ${ }^{1}$ According to our previous study, frames within the first few seconds of irradiation "missed dose." The longer the irradiation time, the smaller the relative deficit (proportional to the integrated dose over all frames). The EPID signal per frame persisted in the seconds following beam off, gradually decreasing, indicating image lag. When this "lag" (dark signal) was added to the integrated dose, there was still a deficit. This was attributed to gain ghosting effects. For the purposes of MU dependence, and for the remainder of this paper, we refer to the combination of gain ghosting and image lag as "ghosting." Ghosting effects can cause problems for EPID dosimetry if the imager signal is assumed to be linear with accumulated dose. Discrepancies will arise when the treatment exposure time differs from calibration exposure times.

Other studies, however, have reported a linear dose-signal relationship within $2 \%{ }^{7-12}$ All of these studies used the Varian EPID, which has a different scintillator from the Elekta and Siemens detectors. The EPID signal for these studies was measured over different dose ranges, energies, and dose-rate settings compared to measurements with the Elekta EPIDs. Dosimetric characteristics for the Siemens EPIDs have not yet been reported. Non-linearity due to energy spectrum and dose/frame changes, or differences in acquisition software, can also influence the dosimetric 
characteristics. ${ }^{1,2,5,13}$ The purpose of this study was to compare the signal-to-monitor unit (MU) ratio for a comparable (wide) dose range, for all three $a$-Si EPID brands.

\subsection{MATERIALS AND METHODS}

Six $a$-Si EPIDs were investigated in this study: two Elekta panels (iView GT) from the Netherlands Cancer Institute, Amsterdam, The Netherlands, one Varian panel (aS500) at the Rigshospitalet, Copenhagen, Denmark, another Varian panel (aS500) at The Royal Marsden Hospital, London, United Kingdom, and two Siemens panels (OptiVue 500 and 1000) at the Maastricht University Hospital, Maastricht, The Netherlands. Commercial acquisition software was used to acquire images for the Varian and Siemens EPIDs. In-house software, on the other hand, was used to acquire images with the Elekta EPIDs. This software is very similar to the commercially available acquisition software provided by Elekta for the iView GT detector. ${ }^{14}$ The active detection areas and image resolutions of each panel are given in Table 6.1. The Varian aS1000 was not tested in this study, the difference between this panel and the aS500 is a higher resolution (1024x768 pixels), with the same active area and acquisition software.

Ghosting effects are known to depend on exposure time, ${ }^{5}$ which is linked to the dose-rate for a given dose. The Elekta and Siemens frame acquisition rates are constant, both $\approx 3.5$ frames per second (fps). The Varian acquisition rate depends on the linac pulse rate, which was $\approx 4.5$ to $7.5 \mathrm{fps}$ for the dose-rates measured in this study. One of the differences between the two Varian panels tested in this study was that different versions of Varian's PortalVision software were used to acquire images. The earlier version (v6.1.03, "Varian A") employs a reset every 64 frames to move the frame buffer content to the CPU, creating a dead time of $0.28 \mathrm{~s}$, or loss of one to two image frames (depending on the frame rate). ${ }^{10}$ More recent versions of the software do not have this dead time.

For each panel, images were acquired for a series of open square fields, irradiated with $5,10,20,50,100,200,500$, and 1000 MUs, integrated over all frames. Various dose-rate and photon beam energies settings were tested, according to the available settings for each linac on which the panels were mounted. For the Elekta and Varian detectors, eight series were measured ( $A$ and B EPIDs, each with two dose-rate/beam energy combinations, each series measured twice). For the Siemens detectors, six series were included. "Siemens $A$ " was measured with two dose-rate/beam energy combinations, and "Siemens B" with four dose-rate/beam energy combinations. Details regarding panel properties, beam parameters, and image acquisition parameters are summarized in Table 6.1. Measurements with the Elekta panel were performed first, with field size $20 \times 20 \mathrm{~cm}^{2}$ and source-detector distance $(S D D)=160 \mathrm{~cm}$. Measurements with subsequent detectors could not be made with the same parameters because the dimensions of the panels and the SDDs (and hence effective field size at the detector) varied at other clinics. All fields were much larger than the central region of interest (ROI) selected for analysis (by more than a factor of 8), to avoid any field edge effects. The results were expressed as the EPID signal divided by 


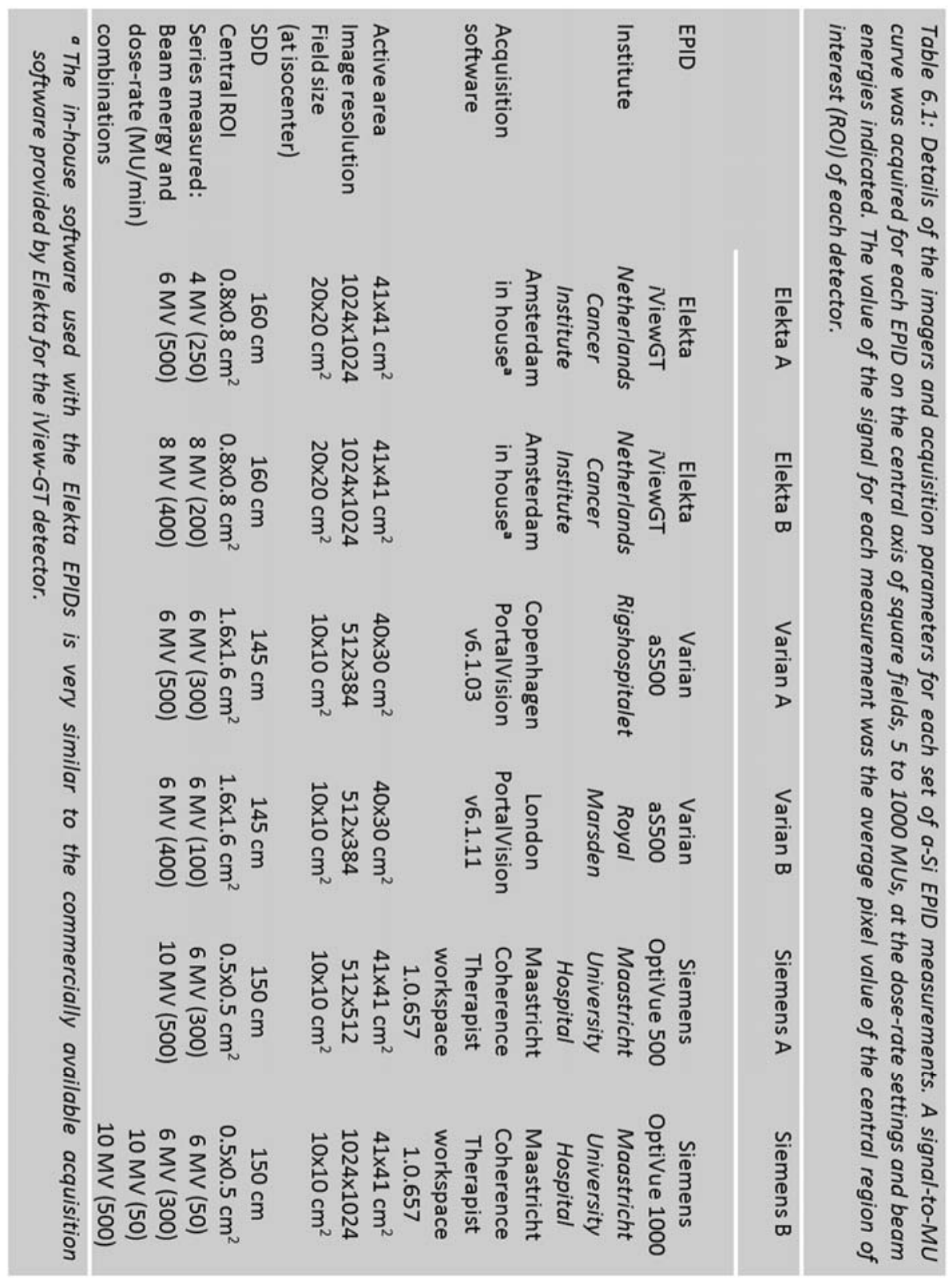


the number of MUs and then normalized to the ratio at 1000 MUs. It should be noted that only individual, non-segmented square fields were investigated to be able to compare the EPIDs without introducing too many variables. The implications of ghosting effects for IMRT fields (segmented or dynamic) fall outside the objectives of this study.

\subsection{RESULTS}

Figure 6.1 shows an average of the series measured for each of the three $a$-Si EPID brands. For the Varian EPID, only four series using "Varian B" were included in the average (two dose-rate/beam energy combinations, each series measured twice). The measurements with "Varian A" were not included here because it uses a different acquisition software, however it is presented separately. All series exhibited a lower signal-to-MU ratio for shorter irradiation times. This is consistent with previous reports suggesting that ghosting effects depend on exposure and/or acquisition time. ${ }^{1,5}$ For irradiations of more than $200 \mathrm{MUs}$, the ratio for each detector was constant to within $\pm 1.5 \%$, i.e., the response is effectively linear with dose. Below $200 \mathrm{MUs}$, the average signal-to-MU ratio decreases $4 \%$ for the Elekta panels, and $5 \%$ for the Varian and Siemens panels.

Error bars represent \pm 1 standard deviation (SD). The relative average SD was $0.3 \%$ and the maximum was $\pm 1.4 \%$. As expected, the results averaged over the largest range of dose-rate/beam energy combinations had the largest SD, i.e., the Siemens dose-rate settings, ranging from 50 to $500 \mathrm{MU} / \mathrm{min}$, with beam energies of 6 and $10 \mathrm{MV}$. A variation in the signal-to-MU ratio could be due to variation in the design and manufacture of the a-Si layer, (as used by different brands), or read-out of the electronics, leading to a different number of charge particles trapped and/or read out in the bulk modulus or interface of the photodiode layer. In addition to physical differences, different image acquisition parameters (e.g., trigger levels) will also influence the EPID signal differently at various exposure times.

Signal-to-MU ratios measured at different beam energies and dose-rate settings for each detector are also shown in Fig. 6.2. For each detector type, the MU dependence was similar (within $\pm 1.4 \%$ ) for all energies and dose-rate settings, except below 10 MUs for the Varian A and B EPIDs.

For the EPID using the earlier version of PortalVision (Varian A), the signal-to-MU curve dropped by $1 \%$ between 50 MUs (43 frames) and 100 MUs (95 frames), for both dose-rates. The discontinuity in the curve was due to the reset occurring every 64 frames and so resulted in a dead time during acquisition if more than 64 frames were acquired (Fig. 6.2). The data for both Varian A series were subsequently corrected for the missing signal due to dead time and are also given in Fig. 6.2. The difference in signal ratio between 5 and 1000 MUs is clearly much greater for the corrected Varian A than Varian B. The reason was not investigated further for this study, however it can be assumed that differences in image acquisition, panel design, and variation in 


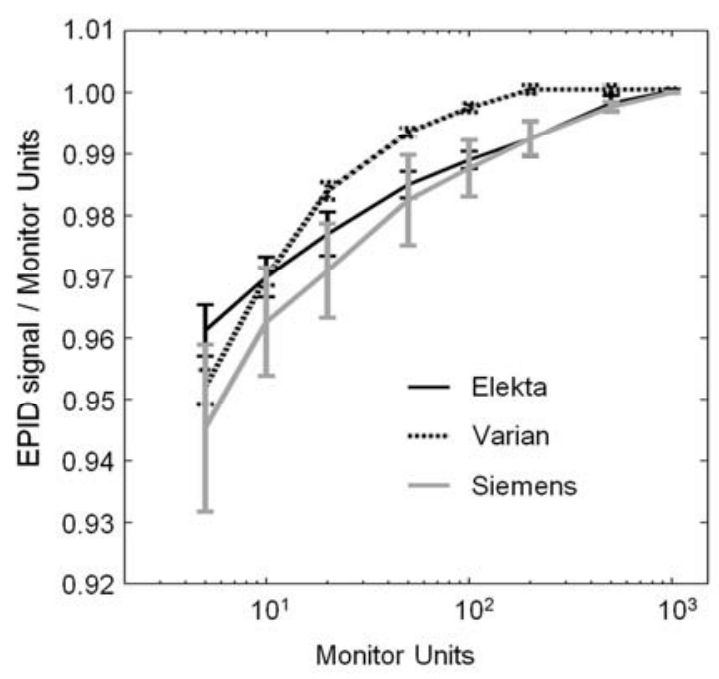

Figure 6.1: Signal-to-MU ratios for Elekta, Varian, and Siemens a-Si EPIDs, averaged over two to three dose-rate settings for different energies, with one or two detectors for each brand. All points are normalized at 1000 MUs. One outlying series, Varian A, used a different acquisition mode and was therefore excluded for this figure. The standard deviations at each point were less than $1.4 \%$, and are shown as error bars ( \pm 1 SD). Different scintillators employed by different brands will exhibit slight variation in ghosting effects, however there is a consistent under-response for fields of fewer MUs for all three brands.

read-out electronics are possible reasons for the differences between the two sets of measurements in Fig. 6.2.

Due to non-linearity of linac monitor signal, the Siemens EPID signals measured with 5 MUs, $6 \mathrm{MV}$, and $300 \mathrm{MU} / \mathrm{min}$ were corrected based on relative dose values measured with an ionization chamber. The linac output used for all other series was also checked and found to be linear, so no corrections were necessary. Two series were also measured with the "Siemens B" EPID at very low dose-rate settings of $50 \mathrm{MU} / \mathrm{min}$. The relative signal-to-MU ratio at smaller number of MUs (0.96 at $5 \mathrm{MUs}$ ) was not as low as for the higher dose-rate settings ( 0.93 at $5 \mathrm{MUs}$, same EPID, same beam energies). This dose-rate dependence is consistent with ghosting behavior. Since ghosting depends on the exposure time and not on dose, slower dose-rates will result in an EPID signal with a much weaker MU dependence. This is because a lower nominal dose-rate setting at the linac will result in a lower dose per frame rate. At lower dose per frame rates, an equilibrium can be achieved much faster between the amount of charge that is trapped, and the amount that is read out. So at very low dose-rates, there would be no ghosting effect. The range of dose-rate settings for the Elekta and Varian panels was not large enough to see this effect. It should be noted that although all measurements were normalized to the respective EPID signals at $1000 \mathrm{MUs}$, there was variation in the EPID signal at this normalization point for different dose-rates of the order of a few 

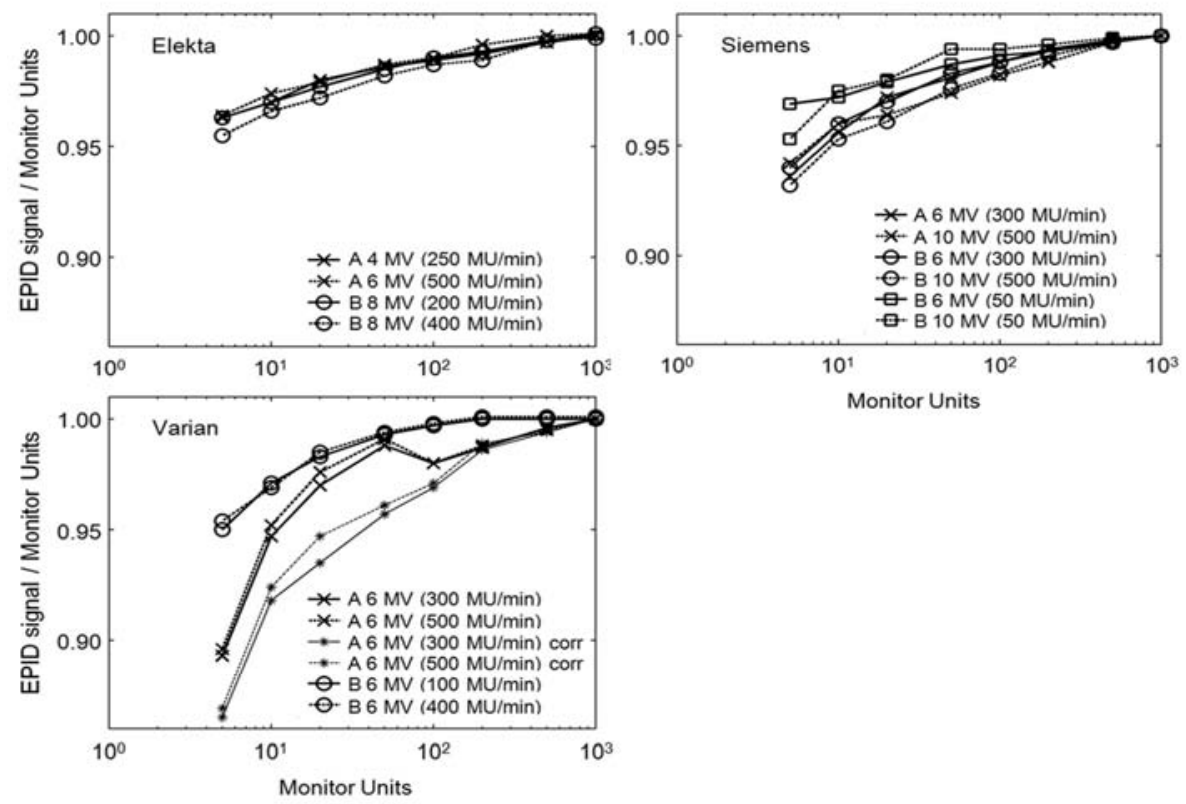

Figure 6.2: EPID signal-to-MU ratios, separated for Elekta, Varian, and Siemens detectors. Two panels ( $A$ and $B$ ) for each brand were tested and normalized at 1000 MUs. The curves are similar for all but one series. For the EPID using the earlier version of PortalVision (Varian $A$, " $x$ "), there is a 1\% "drop" in the curve, between 50 and 100 MUs, for both dose-rates. This discontinuity is due to a dead time introduced while frames are being stored, occurring every 64 frames. The data for both Varian A series are also presented, correcting for the missing signal ("*”).

percent (for the same detector brand, linac, and energy). This difference could be best illustrated with additional data at multiple dose-rate settings for each detector, linac, and energy combination, however this is beyond the scope of this technical note.

\subsection{CONCLUSIONS}

Signal-to-MU ratios for all EPIDs tested showed a dependence on the number of MUs delivered, independent of the manufacturer. This dependence indicated that charge trapping, resulting in ghosting effects, influences the $a$-Si EPID response to dose. Therefore it is important to be aware of the resulting relative under-response at shorter irradiation times. The similarity of the results for all detectors tested suggested that the acquisition time dependence, or ghosting effect, is a fundamental property of indirect-detection $a$-Si-based EPIDs. The small differences between the signal-to-MU ratio for the three manufactures was likely to be due to differences in panel design and acquisition software. Variation between curves of the same manufacturer may be due to a combination of dose-rate and energy dependence, both influencing the dose delivered per frame. Errors of $4-10 \%$ at the center of the field are likely to influence 
126 Chapter 6

EPID dosimetry measurements if the imager is applied over a wide range of irradiation times, by varying dose or dose-rate, to single fields without corrections.

\section{ACKNOWLEDGEMENTS}

The authors would like to thank Håkan Nyström and Marika Björk of The Finsen Centre, Rigshospitalet, Copenhagen, Denmark for permission to use their equipment and assistance with measurements. This work was financially supported by the Dutch Cancer Society (Grant No. NKI 2000-2255). 


\section{REFERENCES}

1 L.N. McDermott, R.J. Louwe, J.J. Sonke, M.B. van Herk, and B.J. Mijnheer, "Dose-response and ghosting effects of an amorphous silicon electronic portal imaging device," Med. Phys. 31, 285-295 (2004).

2 P. Winkler, A. Hefner, and D. Georg, "Dose-response characteristics of an amorphous silicon EPID," Med. Phys. 32, 3095-3105 (2005).

3 J.H. Siewerdsen and D.A. Jaffray, "A ghost story: Spatio-temporal response characteristics of an indirect-detection flat-panel imager," Med. Phys. 26, 1624-1641 (1999).

4 M. Overdick, T. Solf, and H. Wischmann, "Temporal artefacts in flat dynamic x-ray detectors," Proc. SPIE 4320, 47-54 (2001).

5 M. Partridge, B.M. Hesse, and L. Müller, "A performance comparison of direct- and indirect-detection flat-panel imagers," Nucl. Instrum. Methods Phys. Res. A 484, 351-363 (2002).

6 H. Wischmann, H. Luijendijk, H. Meulenbrugge, M. Overdick, R. Schmidt, and K. Kiani, "Correction of amplifier non-linearity, offset, gain, temporal artifacts, and defects for flat-panel digital imaging devices," Proc. SPIE 4682, 427-437 (2002).

7 B.M. McCurdy, K. Luchka, and S. Pistorius, "Dosimetric investigation and portal dose image prediction using an amorphous silicon electronic portal imaging device," Med. Phys. 28, 911-924 (2001).

8 E.E. Grein, R. Lee, and K. Luchka, "An investigation of a new amorphous silicon electronic portal imaging device for transit dosimetry," Med. Phys. 29, 2262-2268 (2002).

9 B. Warkentin, S. Steciw, S. Rathee, and B.G. Fallone, "Dosimetric IMRT verification with a flat-panel EPID," Med. Phys. 30, 3143-3155 (2003).

10 P.B. Greer and C.C. Popescu, "Dosimetric properties of an amorphous silicon electronic portal imaging device for verification of dynamic intensity modulated radiation therapy," Med. Phys. 30, 1618-1627 (2003).

11 G.V. Menon and R.S. Sloboda, "Compensator quality control with an amorphous silicon EPID," Med. Phys. 30, 1816-1824 (2003).

12 A. Van Esch, T. Depuydt, and D.P. Huyskens, "The use of an aSi-based EPID for routine absolute dosimetric pre-treatment verification of dynamic IMRT fields," Radiother. Oncol. 71, 223-234 (2004).

13 Y. El Mohri et al., "Relative dosimetry using active matrix flat-panel imager (AMFPI) technology," Med. Phys. 26, 1530-1541 (1999).

14. B. Brand, J.J. Sonke, and M.B. van Herk, "Synchronising portal images and A/D measurements," Radiother. Oncol. 68, s95-s96 (2003). 



\section{CHAPTER}

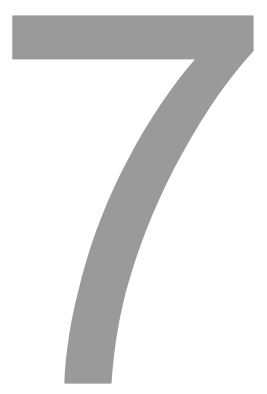

Prediction of DVH parameter changes due to set-up errors for breast cancer treatment based on 2D portal dosimetry

Med. Phys. 36(1), 83-94 (2009)

S.M.J.J.G. Nijsten W.J.C. van Elmpt B.J. Mijnheer A.W.H. Minken L.C.G.G. Persoon P. Lambin A.L.A.J. Dekker 



\section{ABSTRACT}

Electronic portal imaging devices (EPIDs) are increasingly used for portal dosimetry applications. In our department, EPIDs are clinically used for two-dimensional (2D) transit dosimetry. Predicted and measured portal dose images are compared to detect dose delivery errors caused for instance by set-up errors or organ motion. The aim of this work is to develop a model to predict dose-volume histogram (DVH) changes due to set-up errors during breast cancer treatment using 2D transit dosimetry. First, correlations between DVH parameter changes and 2D gamma parameters are investigated for different simulated set-up errors, which are described by a binomial logistic regression model. The model calculates the probability that a DVH parameter changes more than a specific tolerance level and uses several gamma evaluation parameters for the planning target volume (PTV) projection in the EPID plane as input. Second, the predictive model is applied to clinically measured portal images. Predicted DVH parameter changes are compared to calculated DVH parameter changes using the measured set-up error resulting from a dosimetric registration procedure. Statistical accuracy is investigated by using receiver operating characteristic (ROC) curves and values for the area under the curve $(A U C)$, sensitivity, specificity, positive and negative predictive values. Changes in the mean PTV dose larger than $5 \%$, and changes in $V_{90}$ and $V_{95}$ larger than $10 \%$ are accurately predicted based on a set of $2 \mathrm{D}$ gamma parameters. Most pronounced changes in the three DVH parameters are found for set-up errors in the lateral-medial direction. AUC, sensitivity, specificity, and negative predictive values were between $85 \%$ and $100 \%$ while the positive predictive values were lower but still higher than $54 \%$. Clinical predictive value is decreased due to the occurrence of patient rotations or breast deformations during treatment, but the overall reliability of the predictive model remains high. Based on our predictive model, 2D transit dosimetry measurements can now directly be translated in clinically more relevant DVH parameter changes for the PTV during conventional breast treatment. In this way, the possibility to design decision protocols based on extracted DVH changes is created instead of undertaking elaborate actions such as repeated treatment planning or 3D dose reconstruction for a large group of patients. 


\subsection{INTRODUCTION}

In radiotherapy, electronic portal imaging devices (EPIDs) have been used for patient position verification for many years now. A more recent application of EPIDs is to use them for portal dosimetry by measuring non-transmission or transmission portal dose distributions. Non-transmission images can be measured pre-treatment and are used to verify treatment parameter transfer between treatment planning system (TPS) and treatment machine, ${ }^{1}$ but also to detect malfunctioning of the treatment machine. Especially in the case of intensity-modulated radiation therapy (IMRT) treatments, ${ }^{2-5}$ this verification procedure is important because a high accuracy of the linear accelerator and multileaf collimator is required. Transmission images are measured in the presence of a patient and allow for detection of dose differences caused for instance by set-up errors and organ motion. For both types of images, the portal dose can be measured in the EPID plane ${ }^{1-3,6,7}$ but also reconstructed inside a patient in two or three dimensions. ${ }^{4,5,8,9}$

In our department, we are clinically performing two-dimensional (2D) transit dosimetry for all patients treated with curative intent. This procedure compares predicted and measured portal dose images (PDIs) in the EPID plane by using the gamma evaluation method ${ }^{10,11}$ and accepts or rejects images applying criteria based on gamma (evaluation) parameters. Because PDIs only contain projection information, dose differences cannot be related directly to differences in the 3D dose distribution inside a patient. By using a 3D dose reconstruction procedure, ${ }^{12}$ changes in the patient dose distribution and the corresponding dose-volume histograms (DVHs) can be calculated but this procedure is more complex and time-consuming. Preferably, a faster method like 2D transit dosimetry is used as a triage for the full 3D dose reconstruction.

Therefore, the aim of this work was to develop a model to predict quantitatively DVH parameter changes based on 2D transit dosimetry. The model has been implemented for conventional breast treatments where large dose differences may occur due to the presence of set-up errors. The accuracy of the model has been determined by applying it to clinically obtained gamma images.

\subsection{MATERIALS AND METHODS}

\subsubsection{Equipment}

In our department, six Siemens Oncor medical linear accelerators (Siemens Medical Solutions, Concord, CA) are applied for patient treatment in combination with Siemens OptiVue 500/1000/1000 ST amorphous silicon ( $a$-Si) flat panel portal imagers (Siemens Medical Solutions, Concord, CA). All accelerators are equipped with 6 and $10 \mathrm{MV}$ photon beam energies, a virtual wedge option, and are dosimetrically matched within $1 \%$ (1 SD) when depth-dose curves, beam profiles, and output factors are compared. 


\subsubsection{Extraction of 2D gamma parameters}

In this work, dose-volume histogram changes due to set-up errors during breast cancer irradiations are predicted based on differences in portal dose images behind a patient (transit PDIs). Both predicted and clinically obtained PDIs for multiple values of set-up errors are used for this purpose (Sec. 7.2.2.1-2), which are compared to a predicted reference PDI without any set-up error; the comparison is based on the gamma evaluation method. ${ }^{10,11}$ Gamma images are analyzed to obtain a set of 2D gamma parameters which can be used to predict DVH changes (Sec. 7.2.2.3). In this work, only DVH changes for the planning target volume (PTV) are investigated and portal dose images are generated at a source-detector distance of $150 \mathrm{~cm}$, resulting in images of $512 \times 512$ pixels with a pixel resolution of $0.8 \mathrm{~mm}$.

The programming language MATLAB 2007b (The MathWorks Inc., Natick, MA) is used for all calculations, modeling, and analysis, unless mentioned otherwise.

\subsubsection{Prediction of 2D portal dose images}

A portal dose prediction model based on pencil beam scatter kernels is applied to predict a 2D full-scatter transit portal dose image and has been described elsewhere in more detail. ${ }^{13}$ The model uses a predicted full-scatter portal dose image without the patient in the beam in combination with the radiological thickness of a patient derived from the planning CT scan. Additional information such as gantry angle, couch angle, and isocenter position is obtained from the treatment plan. The portal dose prediction model is fitted to one measurement set per photon beam energy and is machine-independent. Based on dose in water measurements behind homogeneous and inhomogeneous phantoms, the accuracy of the model is better than $2 \%$ (mean difference). Portal dose images for different set-up errors (patient shifts) are calculated by moving the isocenter of a beam in the opposite direction in the planning CT scan.

\subsubsection{Measurement of 2D portal dose images}

A dosimetric calibration model for $a$-Si EPID image to obtain a measured 2D full-scatter transit portal dose image by taking into account all relevant EPID response corrections. This model has been developed in our department and has been described elsewhere. ${ }^{14}$ The accuracy of the dosimetric calibration model has been determined by performing measurements in a water tank for different treatment fields and (in)homogeneous phantoms using the gamma evaluation method. In almost all situations, the observed gamma values were smaller than 1 using $3 \%$ as the dose-difference criterion and $3 \mathrm{~mm}$ as the distance-to-agreement criterion. The dosimetric calibration model is fitted to a measurement set per photon beam energy and per treatment machine. 


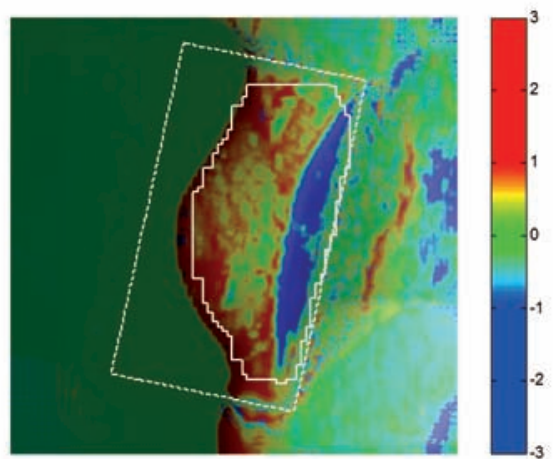

(a)

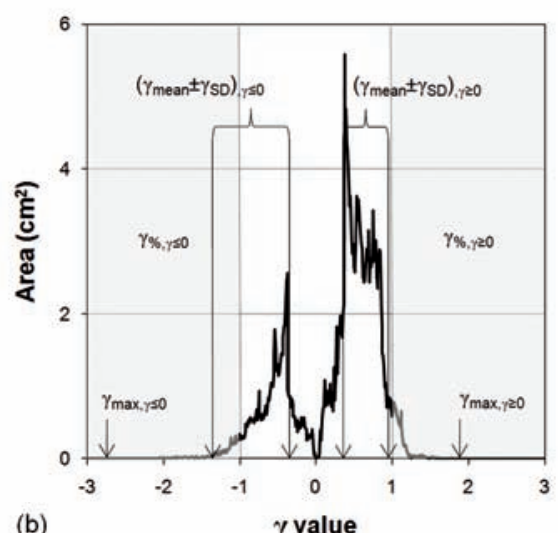

(b)

Figure 7.1: (a) A gamma image based on a transit PDI with a set-up error of $5 \mathrm{~mm}$ in left-right direction and a transit PDI without set-up error, plotted on top of a DRR. Gamma values are calculated using $3 \%$ as local dose-difference criterion and $3 \mathrm{~mm}(4.5 \mathrm{~mm}$ at $150 \mathrm{~cm})$ as distance-to-agreement criterion. These gamma values can be smaller than 0 , allowing an easy visualization of PDI regions where the portal dose in presence of a set-up error is smaller (blue regions) or larger (red regions) than the portal dose without any set-up error. The field shape is represented by the dashed white line. (b) From the gamma image shown in (a), the 2D gamma parameters $\gamma_{\text {max }} \gamma_{\text {mean }} \gamma_{S D}$ and $\gamma_{\%}$ were calculated within the PTV projection (solid white line) both for $\gamma$ values smaller and larger than 0 . For this example, the parameter values are $\gamma_{\max , y \leq 0}=-2.8$, $\gamma_{\text {max }, \gamma \geq 0}=1.9,\left(\gamma_{\text {mean }} \pm \gamma_{S D}\right)_{, \gamma \leq 0}=-0.9 \pm 0.5,\left(\gamma_{\text {mean }} \pm \gamma_{S D}\right)_{, \gamma \geq 0}=0.7 \pm 0.3, \gamma_{\%, \gamma \leq 0}=13.8 \%$ and $\gamma_{\%, \gamma \geq 0}=8.2 \%$.

\subsubsection{Calculation of 2D gamma parameters}

The influence of set-up errors on a portal dose distribution has been investigated by calculating a gamma image based on a predicted transit PDI without any set-up error and a predicted or measured transit PDI with set-up error. An example of a gamma image is shown in Fig. 7.1(a) for a predicted PDI with a set-up error of $5 \mathrm{~mm}$ in the left-right direction. In this work, gamma values are calculated using $3 \%$ as the local dose-difference criterion and $3 \mathrm{~mm}(4.5 \mathrm{~mm}$ at $150 \mathrm{~cm})$ as the distance-to-agreement criterion.

From the gamma images, several 2D gamma parameters are calculated: the maximum $\gamma$ value $\left(\gamma_{\text {max }}\right)$, the mean $\gamma$ value $\left(\gamma_{\text {mean }}\right)$, the standard deviation of the mean $\gamma$ value $\left(\gamma_{S D}\right)$, and the percentage of $\gamma$ values larger than $1\left(\gamma_{\%}\right)$. The maximum $\gamma$ value is defined in this article as the highest value of all pixels and not as a specific percentage of pixels, e.g., $1 \%$ having the highest $\gamma$ values. Because a gamma evaluation minimizes gamma values, our definition is still insensitive to large local portal dose values. Furthermore, a distinction is made between $\gamma$ values smaller and larger than 0 . When the dose difference is smaller than 0 , the gamma value is smaller than 0 , which means that the portal dose in the presence of a set-up error is smaller than the portal dose without a set-up error. In this case, a minus sign is added to the $\gamma$ value and the notation is changed, e.g., the 2D gamma parameter $\gamma_{\max }$ becomes $\gamma_{\max , y \leq 0}$. In Fig. 1(b), the different 
2D gamma parameters are shown in a gamma-area histogram. Because 2D gamma parameters are used to predict DVH changes for the PTV, only the PTV projection in the EPID plane is analyzed. This PTV region is calculated for the original PTV without set-up error. Based on eight individual 2D gamma parameters $\left(\gamma_{\text {max }}, \gamma_{\text {mean }}, \gamma_{S D}\right.$, and $\gamma_{\%}$ for both $\gamma \leq 0$ and $\gamma \geq 0$ ), 92 different combinations have been defined consisting of 1,2 , or 3 individual gamma parameters.

\subsubsection{Calculation of DVH parameter changes}

The three-dimensional dose distributions inside a patient have been calculated with our treatment planning system (XiO 4.3.4, Computerized Medical Systems Inc., St. Louis, MO), using a multigrid superposition algorithm. ${ }^{15}$ Simulation of set-up errors is performed by moving the isocenter of a beam in the opposite direction of the patient shift in the planning CT scan and recalculating the 3D dose distribution. Dose-volume histograms are calculated for the PTV only, using a 3D grid spacing of $2 \mathrm{~mm}$.

From the DVHs, nine DVH parameters are calculated: the minimum dose $\left(D_{\min }\right)$, the maximum dose $\left(D_{\max }\right)$, the mean dose $\left(D_{\text {mean }}\right)$, the conformity index $(C l)$, the heterogeneity index $(H I), V_{90}, V_{95}, V_{100}$, and $V_{107}$. The conformity index is the quotient of the treated volume and the PTV; $\mathrm{Cl}$ changes due to set-up errors because the treated volume is influenced. In this work, the $95 \%$ isodose volume is used to define the treated volume in all calculated 3D dose distributions, while the PTV is taken equal to the volume of the $95 \%$ isodose volume in the $3 \mathrm{D}$ dose distribution without any set-up errors imposed $(C l=1)$. The heterogeneity index is the quotient of $D_{\max }$ and $D_{\min }$ for the PTV. Changes in DVH parameters are defined as relative differences with respect to the original DVH parameter value without set-up error and are denoted for instance as $\% D_{\min }$ for the DVH parameter $D_{\min }$.

\subsubsection{Predictive modeling}

Correlations between 2D gamma parameters and DVH parameter changes for the PTV are investigated for 20 patients who were irradiated on the breast gland only with a prescribed dose of $50 \mathrm{~Gy}$ in 25 fractions. Left-and rightsided breast cancer patients are included. Our treatment technique for this target volume basically consists of tangential opposing treatment fields encompassing the entire PTV. The PTV is defined as the macroscopic tumor with $1.5 \mathrm{~cm}$ margin including the breast gland (clinical target volume: CTV) extended with $0.5 \mathrm{~cm}$ margin to account for set-up errors and organ motion, except for the medial-lateral direction where a flash region of $2-4 \mathrm{~cm}$ width is used. In clinical practice, the treatment fields are set-up by looking at the patient anatomy in the planning CT scan without actually delineating CTV and PTV. To make the 3D dose distribution in a patient more homogeneous, often small fields with a small number of monitor units are added. In this work, portal dose images of 43 large tangential fields that are predominant for the delivered 3D dose distribution in a patient are analyzed; the portal dose images of the latter small fields are excluded from analysis. However, DVHs are calculated based on all fields in the original treatment plan. Simulated patient shifts varied between -10 and $10 \mathrm{~mm}$ in left-right 
$(L-R)$, superior-inferior (S-I), and anterior-posterior (A-P) directions, resulting in 35 different set-up errors per patient. In total, 1505 portal dose images were predicted and $7003 \mathrm{D}$ dose distributions were calculated and further analyzed to obtain the 92 different 2D gamma parameter sets (Sec. 7.2.2.3) and nine different DVH parameter changes (Sec. 7.2.3) per predicted PDI and DVH, respectively.

A binomial logistic regression model (Sec. 7.2.4.1) is applied to predict a relevant DVH parameter change based on the values of $2 \mathrm{D}$ gamma parameters. By doing statistical analysis of the prediction results, those $2 \mathrm{D}$ gamma parameter sets were selected that had a high predictive value for specific DVH parameter changes (Sec. 7.2.4.2).

\subsubsection{Binomial logistic regression model}

A binomial logistic regression model generally predicts a discrete outcome (dependent response variable) from a set of independent predictor variables that can be continuous, discrete, dichotomous, or a mix of any of these. The response variable can take the value 1 with a probability of success $p$, or the value 0 with probability $1-p$. In our case, the response variable is a DVH parameter change, e.g., $\% D_{\min }$. This variable takes the value 1 if $\left|\% D_{\min }\right|>\% D_{\min , t o l}$, and 0 if $\left|\% D_{\min }\right| \leq \% D_{\min , t o l} ; \% D_{\min , t o l}$ is a tolerance level that decides when a change in DVH parameter can be considered clinically relevant and also influences the predictive value of the binominal logistic regression model. In this work, tolerance levels of $3 \%, 5 \%, 10 \%$, and $15 \%$ have been used to determine the discrete response variables. The predictor variables are the $2 \mathrm{D}$ gamma parameter values, e.g., $\gamma_{\max , \gamma \geq 0}, \gamma_{\%, \gamma \leq 0}$, and $\gamma_{\%, \gamma \geq 0}$. For the example variables, the probability $p$ of a relevant change in $D_{\min }$ can now be calculated by

$$
p=\frac{\exp \left(\alpha+\beta_{1} \cdot \gamma_{\max _{\gamma} \geq 0}+\beta_{2} \cdot \gamma_{\%, \gamma \leq 0}+\beta_{3} \cdot \gamma_{\%, \gamma \geq 0}\right)}{1+\exp \left(\alpha+\beta_{1} \cdot \gamma_{\max , y \geq 0}+\beta_{2} \cdot \gamma_{\%, \gamma \leq 0}+\beta_{3} \cdot \gamma_{\%, \gamma \geq 0}\right)}
$$

where $\alpha$ is the constant of the equation and $\beta$ is a coefficient per predictor variable. Based on the data of all 20 patients, these coefficients are optimized for the 92 possible 2D gamma parameter sets per individual DVH parameter.

\subsubsection{Statistical analysis}

Equation (7.1) predicts the probability that a DVH parameter change is larger than the tolerance level. By using a cutoff value $p_{\text {cutoff, }}$ the probability $p$ can be rounded to 0 $\left(p<p_{\text {cutoff }}\right)$ or $1 \quad\left(p \geq p_{\text {cutoff }}\right)$. This cutoff value is determined by using a cross-validation-based procedure, ${ }^{16}$ which means here that 10 times different training sets of 15 patients and validation sets of 5 patients are constructed from the complete set of 20 patients. The predictive model is fitted to the training data sets and applied to the validation data sets. For different cutoff values, predicted outcome was compared to actual outcome which allows the calculation of sensitivity (SE), specificity $(S P)$, positive predictive value ( $P P V)$, and negative predictive value (NPV). Furthermore, receiver operating characteristic (ROC) curves $^{17}$ are computed (showing SE versus 1-SP) where the area under the curve $(A \cup C)$ quantifies the accuracy of a predictive model. In this work, we expressed $A U C, S E, S P, P P V$, and $N P V$ in percentages (meaning that the 
actual area under the curve is divided by 100 ). An AUC of $100 \%$ represents a perfect predictive model; an area of $50 \%$ represents a predictive value dominated by pure chance. The optimal cutoff value is found by aiming at the highest values for $S E$ and $S P$ where a high $S E$ is more important than a high $S P$ to avoid a high percentage of false negative predictions. By taking the mean of all ten optimized cutoff values, a more robust and validated cutoff value $p_{\text {cutoff }}$ is obtained.

The predictive model fits from Sec. 7.2.4.1 are applied in combination with the mean cutoff values to predict relevant DVH parameter changes for the 1505 observations. The same cutoff values are used for the ten fits from the cross-validation-based procedure to predict outcome for the training data sets and validation data sets separately. The selection of the most predictive 2D gamma parameter sets, DVH parameters, and tolerance levels is done by aiming at the highest values for the mean values of $A U C, S E, S P$, and NPV calculated from the ten validation data sets; $P P V$ is found less important but it still has to be larger than 50\%. A smaller PPV means that more PDIs have to be examined in clinical practice, unnecessarily increasing workload, but less real relevant DVH parameter changes will be missed. Minimum required values for $A U C, S E, S P$, and $N P V$ are $90 \%, 90 \%, 80 \%$, and $90 \%$, respectively.

\subsubsection{Clinical verification}

Although the cross-validation-based procedure in Sec. 7.2.4 inherently implies a validation procedure, we will validate our predictive model in clinical practice. Therefore, EPID images measured during treatment of ten breast cancer patients are converted to 2D full-scatter transit PDIs by using the dosimetric calibration model described in Sec. 7.2.2.2 and compared to predicted transit PDIs without any set-up error using a gamma evaluation. From the gamma images, 2D gamma parameters were derived and DVH parameter changes predicted. The frequency of EPID image acquisition was prescribed by clinical protocol and was not daily.

To verify the predicted outcomes, reference outcomes are needed. These are obtained by deriving set-up errors from the measured portal dose images and directly calculating the DVH parameter changes with our treatment planning system based on those set-up errors (Sec. 7.2.3). Set-up errors or patient shifts are determined by application of a dosimetric registration procedure. This procedure iteratively changes patient shift values while minimizing $\gamma_{\%, \gamma \leq 0}$ and $\gamma_{\%, \gamma \geq 0}$, which are calculated from a gamma image based on the measured PDI and a newly predicted PDI with those patient shift values. Gamma values are analyzed within the treatment field minus a 5 $\mathrm{mm}$ margin to the field edges, which means that all available dosimetric information is used by the registration procedure. Both the influence of patient rotation and breast deformation on the portal dose distribution are neglected. The registration procedure is sped up by first using patient shifts only parallel to the EPID plane and next perpendicular to it. The patient shifts derived in this way are finally averaged over the treatment fields per treatment session and used for calculating reference DVH parameter changes but also for prediction of 2D transit PDIs. Extraction of 2D gamma parameters based on these PDIs and a predicted transit PDI without any set-up error is done to predict again DVH parameter changes. However, these changes are now 

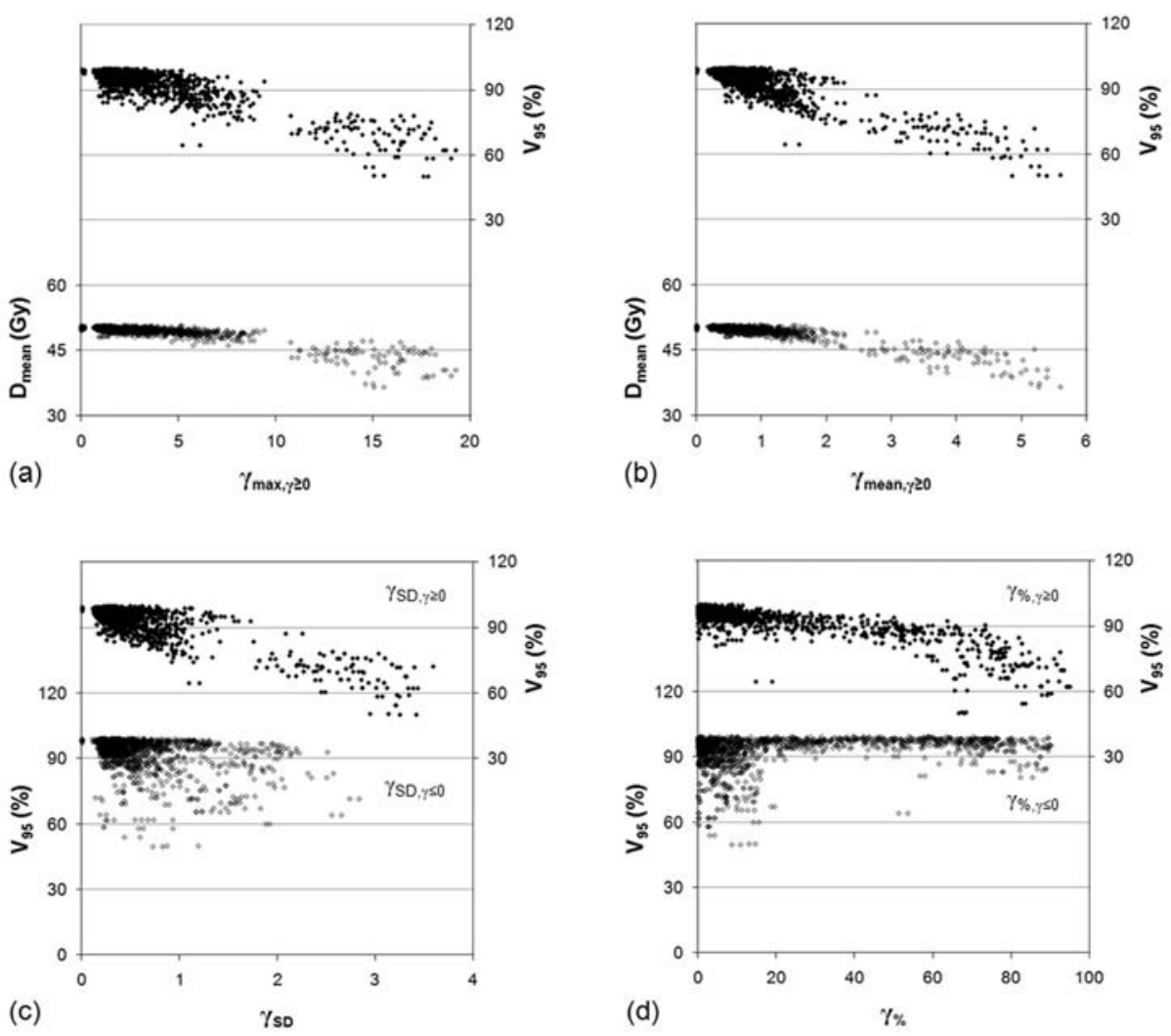

Figure 7.2: Correlations between 2D gamma parameters and DVH parameters for 43 tangential breast treatment fields and 35 different set-up errors per patient, resulting in 1505 observations. $D_{\text {mean }}$ and $V_{95}$ are plotted versus (a) $\gamma_{\text {max }, y \geq 0}$ and (b) $\gamma_{\text {mean }, y \geq 0}$. In (c) and (d), correlations are shown for $V_{95}$ as a function of $\gamma_{S D}$ and $\gamma_{\%}$, respectively.

purely caused by set-up errors and not by patient rotations or breast deformations, which can be the case for the measured transit PDIs.

The accuracy of our predictive model has been determined for the prediction of DVH parameter changes by evaluating values of $A U C, S E, S P, P P V$, and NPV again.

\subsection{RESULTS}

\subsubsection{Correlations}

In Fig. 7.2, correlations are shown between DVH parameters and single 2D gamma parameters, based on the analysis of 1505 portal dose images and 700 3D dose distributions with varying patient shifts. Both $D_{\text {mean }}$ and $V_{95}$ are plotted versus (a) 

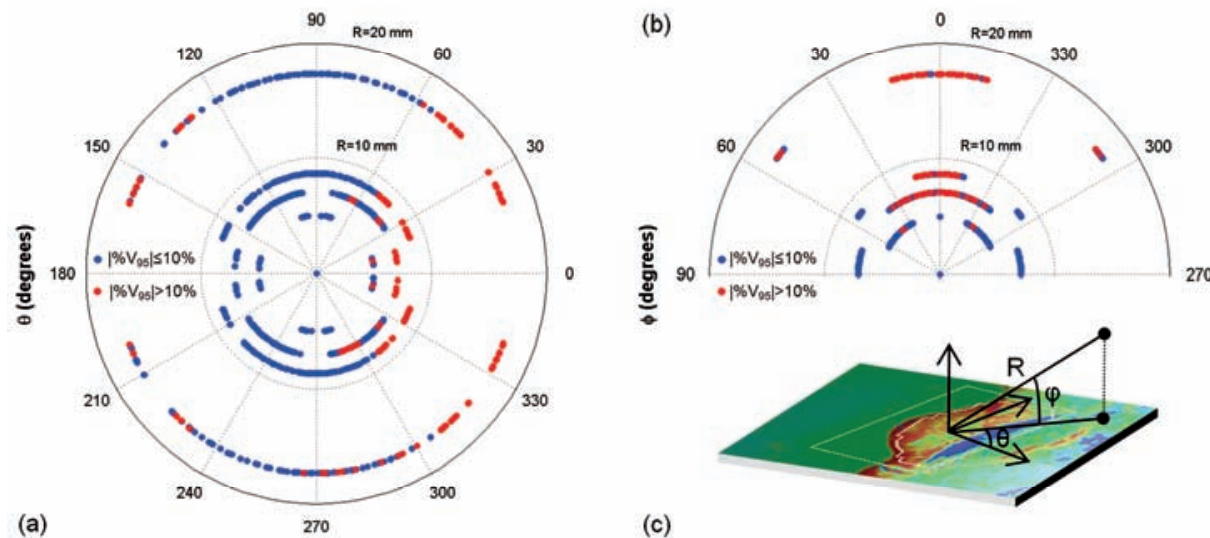

(c)

Figure 7.3: All 1505 simulated patient shifts have been transformed from the patient coordinate system (using L-R, S-I and A-P directions) to the EPID coordinate system as visualized in (c). Two directions are defined in-plane and perpendicular to the field edges; one direction is perpendicular to the EPID plane. The arrows show the directions in which the patient shifts increase $(\geq 0 \mathrm{~mm})$. By using spherical polar transformations, the patient shifts in the EPID coordinate system can be transformed into the radial, azimuth and zenith coordinates denoted by $R, \theta$ and $\varphi$, respectively. The coordinates $\theta$ and $\varphi$ are presented as a function of $R$ in (a) and (b), respectively. For both figures, the data points are shown in blue if the change in DVH parameter $V_{95}$ is smaller than $10 \%$ and in red if the change is larger than $10 \%$. The two in-plane directions are defined with respect to the field edges and therefore to the breast contour. Values for $\theta$ of $0,90,180$ and $270^{\circ}$ correspond with patient shifts in lateral-medial, inferior-superior, medial-lateral and superior-inferior directions, respectively.

$\gamma_{\max , y \geq 0}$ and (b) $\gamma_{\text {mean, } y \geq 0}$, showing a gradual decrease when the two 2D gamma parameters increase. If $\gamma_{\text {max }, y \geq 0}$ is changing from 5 to $10, D_{\text {mean }}$ decreases from $49.3 \pm 0.6$ Gy to $42.3 \pm 3.6$ Gy $(1 \mathrm{SD}) ; V_{95}$ decreases from $91.1 \pm 4.4 \%$ to $65.3 \pm 8.7 \%$ (1 SD). For $\gamma_{\text {mean }, y \geq 0}$ increasing from 1 to 4 , both DVH parameters decrease from $49.8 \pm 0.6$ Gy to $44.6 \pm 1.6 \mathrm{~Gy}(1 \mathrm{SD})$ and from $93.2 \pm 4.4 \%$ to $70.3 \pm 5.5 \%$ (1 SD), respectively. In Figs. 7.2 (c) and 7.2(d), the DVH parameter $V_{95}$ is shown as a function of $\gamma_{S D}$ and $\gamma_{\%}$, respectively, for gamma values smaller and larger than 0 . For $\gamma$ values smaller than 0 , the noise in the data points is increased and it is difficult to distinguish a gradual decrease in $V_{95}$ anymore as a function of the two gamma parameters. In the case of $\gamma$ values larger than 0 , a similar decrease in $V_{95}$ can be observed as in (a) and (b). If $\gamma_{S D}$ increases from 1 to $3, V_{95}$ changes from $88.4 \pm 7.2 \%$ to $67.7 \pm 8.3 \%$ (1 SD) using $\gamma_{S D, \gamma \geq 0}$. In case of $\gamma_{\%}$ changing from 20 to $60 \%, V_{95}$ decreases from $90.4 \pm 4.2 \%$ to $81.6 \pm 7.8 \%$ (1 SD) for $\gamma_{\%, \gamma \geq 2}$. For $\gamma_{S D, \gamma \leq 0}$ and $\gamma_{\%, \gamma \leq 0}$, no significant changes could be observed.

Correlations between set-up errors and DVH parameter changes are shown in Fig. 7.3 for the DVH parameter $V_{95}$. The set-up errors or patient shifts are expressed in the EPID coordinate system as shown in Fig. 7.3(c). Most changes in $V_{95}$ larger than $10 \%$ are found around $\theta=0^{\circ}$ and $\varphi=0^{\circ}$, which means that the breast is shifted out of the treatment field in the lateral-medial direction. These changes already occur for $R=5$ 

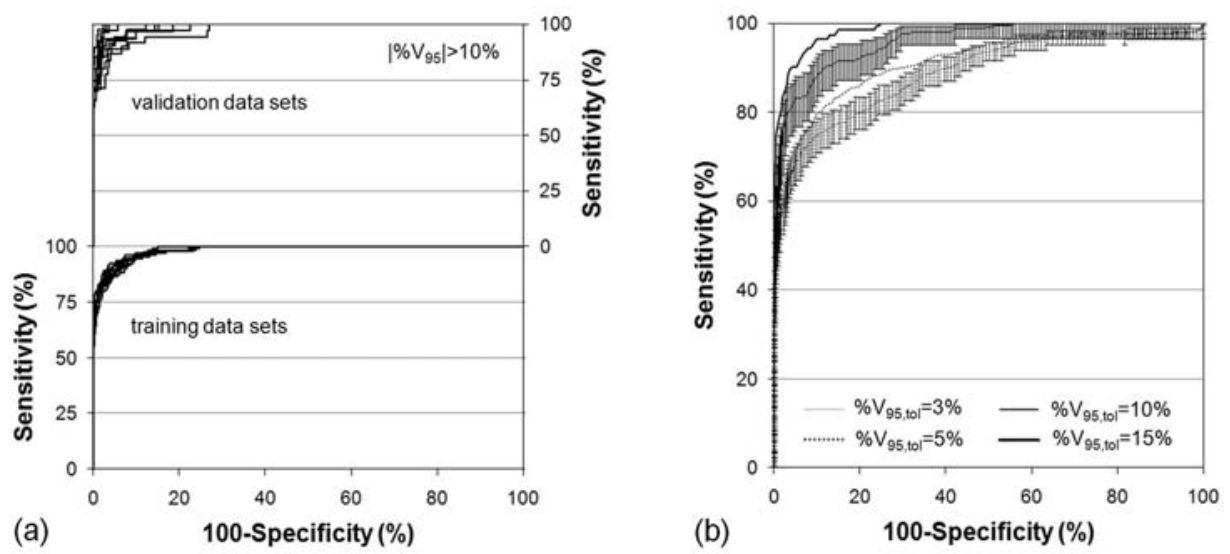

Figure 7.4: Receiver operating characteristic curves quantifying the accuracy of a binomial logistic regression model to predict $V_{95}$ changes larger than $10 \%$ based on the $2 D$ gamma parameters $\gamma_{\max , y \geq 0}, \gamma_{\%, \gamma \leq 0}$ and $\gamma_{\%, \gamma \geq 0}$. (a) ROC curves for 10 mixtures of 15 training data sets and 5 validation data sets resulting from the cross-validation-based procedure where the predictive model coefficients are fit to the training data sets. (b) ROC curves for all patient data sets obtained by applying a predictive model fit based on the data of all 20 patients for different tolerance levels $\% V_{95, \text { tol }}$ of $3 \%, 5 \%, 10 \%$ and $15 \%$. For $\% V_{95, \text { to }}=3 \%$ and $\% V_{95, \text { tol }}=10 \%$, ROC curves are shown with $95 \%$ confidence intervals.

$\mathrm{mm}$ (vector sum of the three patient shifts). The other predominant patient shift direction is found in the medial-lateral direction $\left(\theta=180^{\circ}\right.$ and $\left.\varphi=0^{\circ}\right)$ but only for larger values of $R$. Changes in $V_{95}$ occur also for shifts in the superior-inferior direction but not as frequent as for the former two directions. Similar results are found based on correlations between set-up errors and changes in $D_{\text {mean }}$ and $V_{90}$. Portal dose differences occur for all shifts in the EPID plane but these are most pronounced in the lateral-medial and medial-lateral directions (not shown).

\subsubsection{Predictive modeling}

In Fig. 7.4, ROC curves are shown for a binomial logistic regression model applied to the 2D gamma parameters $\gamma_{\max , \gamma \geq 0}, \gamma_{\%, \gamma \leq 0}$ and $\gamma_{\%, \gamma \geq 0}$ in order to predict changes in $V_{95}$ larger than 10\%. In Fig. 7.4(a), ROC curves are shown for the ten different training data sets and validation data sets that were used in our cross-validation-based procedure to determine optimum values for $p_{\text {cutoff. }}$ The curves for both data sets show a high predictive value with $A U C$ values of $95.8 \pm 0.6 \%$ and $97.4 \pm 1.8 \%$ (1 SD). In Fig. 7.4(b), ROC curves are shown using different tolerance levels to decide when a change in $V_{95}$ can be considered clinically relevant. For this purpose, predictive model fits are calculated based on the data of all 20 patients with an optimum cutoff value of 0.09 . In this case, AUC values were $89.0 \%, 91.1 \%, 96.1 \%$, and $98.5 \%$ using tolerance levels $\% V_{95, t o l}$ of $3 \%$, $5 \%, 10 \%$, and $15 \%$. Based on the selection criteria described in Sec. 7.2.4.2 and values for $S P, S E, P P V$, and NPV, only the tolerance levels of $10 \%$ and $15 \%$ are acceptable in clinical practice. 


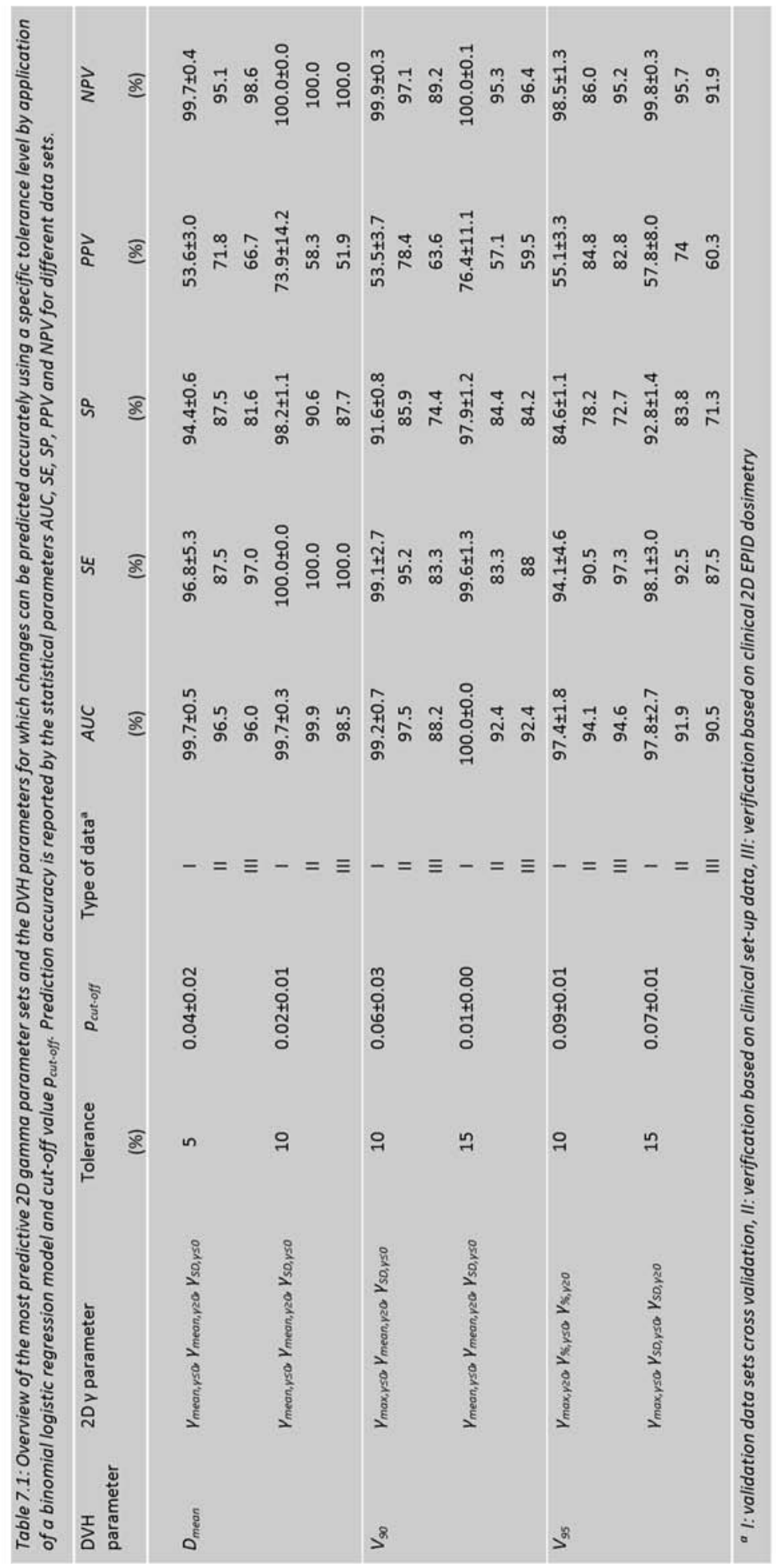




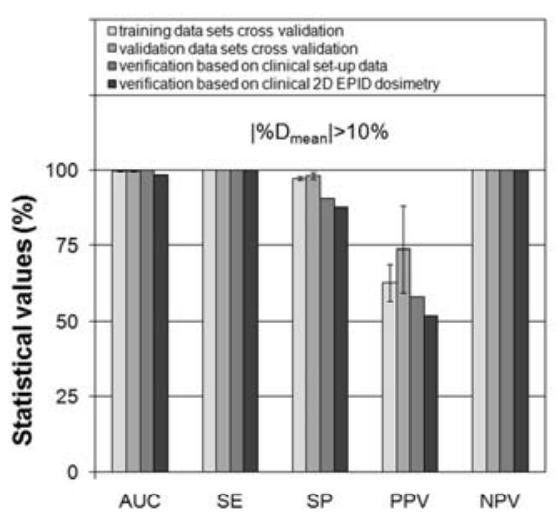

(a)

Statistical parameters

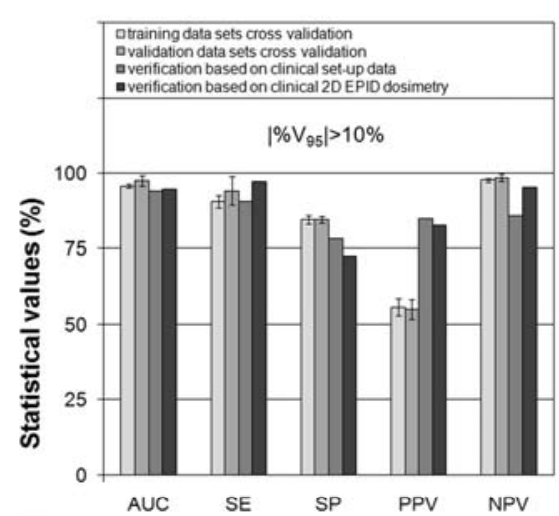

(b)

Statistical parameters

Figure 7.5: Values of AUC, SE, SP, PPV and NPV shown for (a) $\left|\% D_{\text {mean }}\right|>10 \%$ and (b) $\left|\% V_{95}\right|>10 \%$. The $2 D$ gamma parameters $\gamma_{\text {mean, }, \leq 0,}, \gamma_{\text {mean, }, \geq 0,}, \gamma_{S D, \gamma \leq 0}$ and $\gamma_{\text {max }, \gamma \geq 0,}, \gamma_{\%, \gamma \leq 0,}, \gamma_{\%, \gamma \geq 0}$ were used, respectively. The data sets that were analyzed were the training and validation data sets used in our cross-validation-based procedure, and the verification data sets based on clinical set-up data and clinical 2D EPID dosimetry measurements.

An overview of the most predictive and acceptable 2D gamma parameter sets, DVH parameters, and tolerance levels is shown in Table 7.1 for the validation data sets. Besides for $V_{95}$, also relevant changes in $D_{\text {mean }}$ and $V_{90}$ can be predicted based on specific 2D $\gamma$ parameter sets. In the case of $D_{\min }, D_{\max }, \mathrm{Cl}, \mathrm{HI}, V_{100}$, and $V_{107}$, the predictive power was too low to obtain accurate predictions of DVH parameter changes. For $D_{\text {mean }}$, tolerance levels are $5 \%$ and $10 \%$ while for $V_{90}$ and $V_{95}$, values of $10 \%$ and $15 \%$ are derived. If the tolerance level increases, the predictive value of the binomial logistic regression model and particularly the variation in PPV increases. In Fig. 7.5, mean values of $A U C, S E, S P, P P V$, and $N P V$ are shown for (a) $\left|\% D_{\text {mean }}\right|>10 \%$ and (b) $\left|\% V_{95}\right|>10 \%$, corresponding to the training data sets and validation data sets used in our cross-validation-based procedure. The resulting predictive 2D gamma parameter sets consist of three gamma parameters which are calculated for both gamma values smaller and larger than 0 .

\subsubsection{Clinical verification}

Gamma images measured during treatment of a clinical patient are shown in Fig. 7.6 for one of the two tangential breast treatment fields and for different treatment sessions. In Fig. 7.6(a), a predicted transit PDI without set-up error is compared to predicted transit PDIs based on set-up errors determined by our dosimetric registration procedure, while in Fig. 7.6(b) the comparison is done with clinically measured transit PDIs. The two gamma image series show large areas with gamma values both smaller and larger than 0 , meaning that set-up errors or patient shifts are present which are shown in Fig. 7.6(c). The mean set-up errors in L-R, S-I, and A-P directions are $2 \pm 1,-8 \pm 1$, and $-2 \pm 1 \mathrm{~mm}$, respectively. Both gamma image series look very similar but there are also differences in color patterns. During measurement, local 
(a)
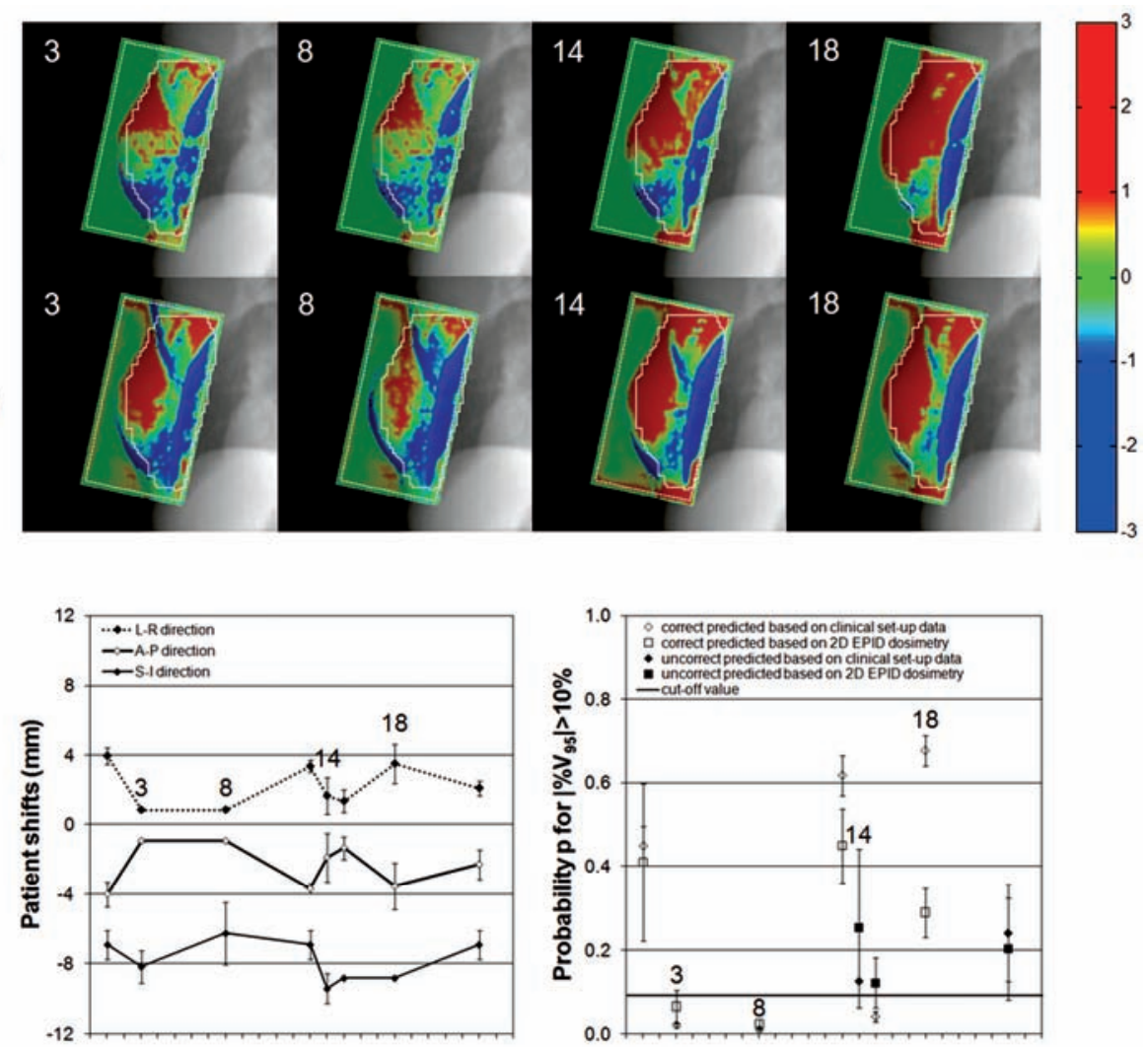

(c)

Treatmentsession

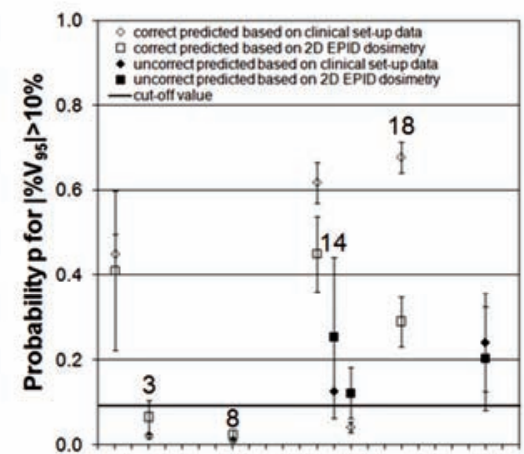

(d)

Treatmentsession

Figure 7.6: Gamma images of a tangential breast treatment field which were calculated by comparison of a predicted transit PDI without set-up error with (a) predicted transit PDIs based on set-up errors determined by a dosimetric registration procedure and (b) clinically measured transit PDIs; treatment sessions 3, 8, 14 and 18 are shown. The static projected PTV regions in the EPID plane are shown, together with the field area that was used for gamma analysis during the registration procedure. The gamma images are plotted on top of DRRs, which incorporate the patient shifts as determined for (a). (c) Patient shifts in the L-R, S-I and A-P directions. (d) The average probability $p( \pm 1 S D)$ that $\left|\% V_{95}\right|>10 \%$ per treatment session in combination with $p_{\text {cutoff }}$ for the predictive model applied to $\gamma_{\max , \gamma \geq 0,}, \gamma_{\%, \gamma \leq 0}$ and $\gamma_{\%, \gamma \geq 0}$ for both tangential fields $(\alpha=-5.68$, $\beta_{1}=0.156, \beta_{2}=0.03$, and $\beta_{3}=0.11$ in equation (7.1)). Open markers indicate correct predicted outcomes while solid markers represent incorrect predicted outcomes, both based on the predicted gamma images using the clinical set-up data (diamond markers) and the measured gamma images using clinical 2D EPID dosimetry (square markers).

gamma differences can be seen at the superior field edges which are not present in the predicted gamma images. Besides this, the red regions in the projected PTV regions can be different in size. In Fig. 7.6(d), the probability $p$ is shown for $\left|\% V_{95}\right|>10 \%$ using the predictive model parameters from Table 7.1 and applied to the two gamma image 
series for both tangential fields. The percentages of correct predicted relevant DVH changes are $75 \%$ and $62.5 \%$ for the predicted and measured gamma images, respectively; the incorrect predicted outcomes for this patient are always false positive predictions.

When looking at Table 7.1 and Fig. 7.5, values for AUC, SE, SP, PPV, and NPV are in general smaller for the clinical verification data sets than for the training and validation data sets used in our cross-validation-based procedure, although there are some exceptions. For the three DVH parameters $D_{\text {mean }}, V_{90}$, and $V_{95}$, values for $P P V$ are often larger for the clinical verification (e.g., predicted $\left|\% V_{95}\right|>10 \%$ based on clinical 2D EPID dosimetry). Comparing the statistical values between the two clinical verification data sets, values for SE and NPV based on measured gamma images can be both smaller and larger than values based on predicted gamma images; values for SP and PPV are mostly smaller in the case of clinical measurements.

\subsection{DISCUSSION}

\subsubsection{Correlations}

Strong correlations between DVH parameters and single 2D gamma parameters are found if gamma values are larger than 0 . Relevant changes in $D_{\text {mean }}, V_{90}$, and $V_{95}$ can be predicted for different tolerance levels if a 2D gamma parameter is included which is calculated for gamma values larger than 0 (Sec. 7.3.2 and Table 7.1). By including 2D gamma parameters for gamma values smaller than 0 in the predictions, the predictive value is increased as shown by the overview of most predictive 2D $\gamma$ gamma parameters in Table 7.1. However, from the correlations presented in Figs. 7.2(c) and $7.2(d)$, the increase in predictive value for $\% V_{95}$ is small by adding $\gamma_{S D, \gamma \leq 0}$ and $\gamma_{\%, \gamma \leq 0}$. Similar results are found when adding $\gamma_{\max , \gamma \leq 0}$ and $\gamma_{\text {mean, } \gamma \leq 0}$, and for $\% D_{\text {mean }}$ and $\% V_{90}$ (not shown).

In this work, 2D gamma parameters are determined by analyzing the projected PTV region in the EPID plane. The 3D PTV is estimated by taking the $95 \%$ isodose volume in the original 3D dose distribution without any set-up error, resulting in a perfectly covered PTV $(\mathrm{Cl}=1)$. This is done because in clinical practice the entire breast gland plus $0.5 \mathrm{~cm}$ margin is considered to be PTV and is not delineated by the physician in the treatment planning system. Probably, the actual PTV is slightly larger because the $95 \%$ isodose surface is usually not completely covering the entire breast gland, especially at ventral and dorsal sides of the lung medially. This means that correlations may slightly change and therefore the predictive model fits. However, we believe that the outcome of this work will not change.

To understand correlations between 2D gamma parameters and DVH parameter changes for the PTV, the influence of set-up errors to both types of parameters has to be discussed. 
In the case of DVH parameter changes, the PTV coverage will change by imposing set-up errors. According to our definition, the conformity index can be both smaller and larger than 1 due to the use of tangential treatment fields encompassing the PTV and the flash region outside the patient contour. In general, values of $D_{\text {mean }}, V_{90}$, and $V_{95}$ decrease due to the simulation of set-up errors because the PTV will shift towards the penumbra or even outside the treatment field. Because tangential fields are used for breast cancer treatment, it is however also possible that the DVH parameters will increase slightly, e.g., if the PTV moves in the medial-lateral direction, causing the treated volume to increase $(C />1)$. Most changes in the three relevant DVH parameters occur for shifts of the breast region out of the treatment field in the lateral-medial direction because the PTV can shift immediately outside the treatment field without any flash region. The same is true for shifts in inferior-superior and superior-inferior directions, but the part of the PTV that moves outside a treatment field in these directions is much smaller than for lateral-medial shifts. On average, lateral and medial field sizes are twice as large as superior and inferior field sizes for tangential breast treatments, and hence also the part of the PTV influenced by set-up uncertainties. Shifts solely in the beam direction do not cause DVH parameter changes when the magnitude of the shift is not too large because the PTV does not move out of the $95 \%$ isodose volume and the PTV coverage remains high. In the case of wedged fields, a shift in the wedge direction can also cause changes in the DVH parameters. For the tangential breast fields, the wedge direction is usually oriented in the lateral-medial direction with a wedge angle between 10 and 30 degrees, having only a small impact on changes in DVH. Furthermore, it can be concluded from Fig. 7.3 that predicting $\left|\% V_{95}\right|>10 \%$ based on set-up errors only is feasible but false positive and false negative predictions will occur.

Local differences in portal dose are found at the treatment field edges but also at the thorax hull or the patient's skin. In gamma images, these differences result in values for $\left|\gamma_{\max }\right|,\left|\gamma_{\text {mean }}\right|, \gamma_{S D}$ and $\gamma_{\%}$ larger than 0 , especially for shifts in the EPID plane. Most portal dose differences are found for lateral-medial and medial-lateral shifts, again due to the larger lateral and medial PTV region sizes. In the case of two opposing shifts in-plane, the gamma parameters $\left|\gamma_{x, \gamma \leq 0}\right|$ for shift 1 and $\left|\gamma_{x, y \geq 0}\right|$ for shift 2 are equal (where $\gamma_{x}$ can be $\gamma_{\text {max }}, \gamma_{\text {mean }}, \gamma_{S D}$ and $\gamma_{\%}$ ), because a gamma evaluation method is a locally applied symmetrical function. In the case of a lateral-medial shift, most gamma values will be larger than 0 , explaining the strong correlation between the $2 \mathrm{D}$ gamma parameters based on these gamma values and the three relevant DVH parameter changes. Gamma values are smaller than 0 for a medial-lateral shift having a small predictive value for DVH parameter changes because the DVH parameters $D_{\text {mean }}, V_{90}$, and $V_{95}$ do not change very much in this direction as explained earlier. These findings show the advantage of our approach to make a distinction between $\gamma$ values smaller and larger than 0 to obtain better correlations between 2D gamma parameters and DVH parameter changes. For shifts in inferior-superior and superior-inferior directions, correlations based on 2D gamma parameters are similar for gamma values smaller and larger than 0 . This explains why the predictive value for a DVH parameter is increased if gamma parameters are included for gamma values smaller than 0 . 
For breast cancer patients, large dose differences have been found earlier by our group ${ }^{6}$ by application of individualized point dosimetry using EPIDs. These dose differences were assigned to the presence of set-up errors up to $1.5 \mathrm{~cm}$ in the lateral-medial direction. In a review, Hurkmans et al. ${ }^{18}$ reported standard deviations (1 SD) of systematic and random set-up errors for breast treatments, ranging from 1.0-4.7 and 1.7-14.4 mm, respectively. Hector et al. ${ }^{19}$ investigated the dosimetric consequences of interfractional patient movement on the accuracy of conventional and IMRT treatments of breast cancer patients. Not only set-up errors but also organ motion was included by determining the change in breast volume during treatment based on regular portal images. However, no correlations were determined between the breast volume, set-up directions, or DVH parameters.

\subsubsection{Predictive modeling}

Based on ten different training data sets, an average value for $p_{\text {cutoff }}$ is found which is directly applied to ten different independent validation data sets. In this way a more robust cutoff value is obtained per individual DVH parameter and tolerance level compared to determining $p_{\text {cutoff }}$ based on the data of all 20 patients without any independent validation. The ROC curves for the training data sets are very similar, which can also be concluded by looking at the small standard deviations in the mean cutoff values in Table 7.1. The training data sets and validation data sets have an excellent agreement when looking at ROC curves and the mean AUC values. Although mean $A U C$ values are slightly larger for the validation data sets, these differences are not statistically significant because the standard deviation in mean AUC values is also increased in the case of the validation data sets.

Instead of using an average predictive model fit based on the logistic regression model determined per individual training data set, predictive modeling is done by using the data of all 20 patients. This approach is supported by the good agreement between the ROC curves for the validation data sets and the ROC curves for all patient data, e.g., $A U C$ values for $\left|\% V_{95}\right|>10 \%$ were $97.4 \pm 1.8$ and 96.1 , respectively.

The predictive value of the model increases when tolerance levels increase, which can be explained by looking at the correlations between 2D gamma parameters and DVH parameter changes. Portal dose differences can be found at the treatment field edges but also at the thorax hull or the patient's skin due to set-up errors. Depending on the magnitude of the set-up error and the gamma criteria values, changes in 2D gamma parameters are less or more pronounced. In this work, gamma evaluation criteria of $3 \%$ and $3 \mathrm{~mm}$ are used, which means that set-up errors of more than $3 \mathrm{~mm}$ can be detected from gamma images. However, DVH parameters for the PTV do not have to change accordingly, as explained in Sec. 7.4.1. When the PTV is still encompassed by the $95 \%$ isodose volume, values of $D_{\text {mean }}, V_{90}$, and $V_{95}$ will not significantly change, which depends on the direction and magnitude of a PTV shift. By increasing the tolerance level, especially the number of false positive predictions is decreased, which improves both SP and PPV but also increases the variation in PPV. Increasing the cutoff 
value $p_{\text {cutoff }}$ for a specific DVH parameter and tolerance level will have the same positive influence to $S P$ and $P P V$ but will increase the number of false negative predictions. In this case, values for SE and NPV will decrease, which is undesirable.

\subsubsection{Clinical verification}

During treatment of ten breast cancer patients, EPID images have been acquired to verify the clinical accuracy of the predictive model. Set-up errors have been determined by using a dosimetric registration procedure that takes into account all available dosimetric information within the treatment field. Hence, the set-up errors do not only describe shifts of the bony structures in a patient but also movement of the breast itself. Patient rotations and breast deformations have been neglected in this work but will influence the direction and magnitude of the observed set-up errors as well. In this work, patient rotation and breast deformations are considered as random errors that blur the 3D dose distribution at the edges of the PTV and will therefore not change DVH parameters significantly. At the EPID plane, however, portal dose differences caused by these rotations and deformations will change the value of 2D gamma parameters and increase the number of false positive predictions. This explains why the values for $S P$ and $P P V$ are in general smaller for the measured gamma images than for the predicted gamma images. For the predicted gamma image series, both gamma images and DVH parameter changes are predicted with the same value of set-up error, which means that no perturbations by patient rotations or breast deformations are introduced. Differences between the two gamma image series are therefore most likely related to the presence of rotations and deformations, e.g., differences visible in Figs. 7.6(a) and 7.6(b) are probably due to a different arm position at the superior field edge and (small) deformations of the breast during treatment.

For the predicted gamma image series, the predictive value should be equal to that for the training and validation data sets but this is not the case. In general, statistical values are smaller except for PPV, which is often larger than for the sets used in our cross-validation-based procedure. This can be explained by looking at the range of simulated and clinically measured set-up errors. During predictive modeling, patient shifts have been varied between -10 and $10 \mathrm{~mm}$ in the L-R, S-I, and A-P directions while the clinically observed patient shifts were in general smaller with mean values of $-2 \pm 3$, $6 \pm 4$, and $3 \pm 3 \mathrm{~mm}$, respectively. Especially the shifts in the L-R and A-P directions sum up to shifts of the breast into the lateral-medial direction causing the largest DVH parameter changes (Sec. 7.4.1). The clinical L-R and A-P shifts are relatively small and will result in 2D gamma parameter values that are less accurate in predicting relevant DVH parameter changes. The number of false positive predictions will be smaller (PPV increases) and the number of true negative predictions effectively decreases even more (SP decreases).

\subsubsection{Clinical applicability}

Minimum tolerance levels for changes in $D_{\text {mean }}$ and the two volume DVH parameters $V_{90}$ and $V_{95}$ were $5 \%$ and $10 \%$, respectively. Based on these tolerance levels, decision protocols can be implemented that are driven by changes in DVH parameters for the 
PTV. Because a transit portal dose image is measured using all dose delivered by the treatment fields, it is not possible to design an on-line decision protocol. However, based on a number of portal dose images measured for different treatment sessions, an off-line decision protocol can be used in clinical practice. In this case, the number of treatment sessions with a clinically relevant change in a DVH parameter, e.g., $\left|\% V_{95}\right|>\% V_{95, t o l}$, can be used to decide if the treatment has to be corrected for set-up or not. Because DVH parameter changes are predicted per beam, conflicting outcomes can be obtained. A possible solution for this is to average the probabilities $p$ from

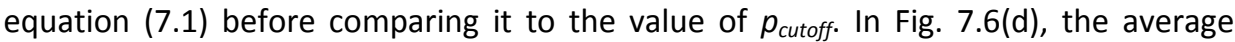
probability $p$ for $\left|\% V_{95}\right|>10 \%$ is shown per treatment session, which behaves completely different in time than the derived patient shifts, as shown in Fig. 7.6(c). Hence, the decision to undertake action based on the predicted DVH parameter changes can also be different compared to the use of set-up errors or 2D gamma parameters, and is more clinically oriented because DVH parameters like $V_{90}$ and $V_{95}$ are directly correlated to treatment outcome or local tumor control.

In Sec. 7.4.3, patient rotations and breast deformations were discussed, which are increasing the number of false positive errors. This means that more PDIs have to be examined in clinical practice and the workload is increased. Dependent on the DVH parameter and tolerance level, the probability of unnecessarily examining a positive PDI is between 0.17 and 0.48 (Table 7.1, data type III, 1-PPV); the probability of missing a real relevant DVH parameter change is smaller than 0.11 (Table 7.1, data type III, $1-N P V)$. In the case of dosimetrical errors like machine output variations, portal dose differences will increase and hence the accuracy of our predictive model will also decrease. To detect these types of errors before patient treatment, we perform a pre-treatment verification procedure in our department where predicted and measured PDIs without the patient in the beam are compared by using the gamma evaluation method.

In this work, only tangential fields from a conventional breast treatment technique are used to determine 2D gamma parameters; DVH parameter changes have been calculated for all fields of a treatment plan. This means that the inclusion of non-tangential single-segment fields will decrease the accuracy of our predictive model. In the case of IMRT, multiple segments are used and this limits the applicability of this work significantly. When a large tangential segment is part of an IMRT field sequence, the accuracy then depends on the relative weight of this tangential segment compared to the other segments. The predictive modeling steps can also be applied to other treatment sites than the breast, with the most important requirement that a class solution is used for the (conformal) treatment technique. Dependent on the treatment technique and chosen beam directions, the prediction of DVH parameter changes for multiple transit portal dose images can be conflicting and predictive accuracy will decrease significantly. For example, in the case of a box technique where four treatment fields are encompassing the PTV, only two of four transit dose portal images will show dose differences if a patient shift is perpendicular to the direction of the two corresponding fields. Hence, DVH parameter changes should be predicted based on the 2D gamma parameter values for all treatment fields, which requires more predictor variables in equation (7.1) and much more simulated patient shifts. 


\subsubsection{Other considerations}

In this work, the four 2D gamma parameters $\gamma_{\text {max }}, \gamma_{\text {mean }}, \gamma_{S D}$ and $\gamma_{\%}$ are calculated to predict relevant changes in DVH parameters for the PTV. However, different 2D gamma parameters can be used to evaluate the agreement of $2 \mathrm{D}$ portal dose images ${ }^{20}$ which possibly can increase the predictive value of the binomial logistic regression model in this work.

The influence of patient rotation on the portal dose images and DVHs is neglected in this work, which decreases the predictive accuracy in clinical practice. Patient rotations can be simulated by varying gantry angle, collimator angle, and couch angle. This refines the correlations and our dosimetric registration procedure will give a more accurate registration result, describing how a patient is actually positioned on the treatment couch during treatment. Furthermore, patient rotation can be distinguished then from breast deformation, although the latter will influence the dosimetric registration accuracy.

Changes in DVH parameters have been predicted for the PTV only and not for the CTV because this was not delineated by the physician in the TPS. From Fig. 7.3, it can be concluded that $\left|\% V_{95}\right|>10 \%$ already occurs for patient shifts of $5 \mathrm{~mm}$ and more in the lateral-medial direction, which is exactly the margin between CTV and PTV. This means that an under-dosage of the CTV (including the breast gland) can indirectly by detected by our predictive model based on the PTV. This work can be repeated to establish correlations between 2D gamma parameters and DVH parameter changes for the CTV if this volume is delineated in the TPS. In this case, slightly different volume parameters (e.g., $V_{97}$ ) might become important.

Although DVH parameters for organs at risk (e.g., lungs and heart) are also important in the case of breast irradiations, no attention has been paid to predicting changes in these parameters. To extend this work for that purpose, accurate predicted and measured portal dose predictions out-of-field are required. However, the feasibility of predicting DVH parameters for lungs and heart is less accurate because distances and shapes of the organs at risk with respect to the 3D dose distribution inside the patient are variable, and the $a$-Si flat panel portal imagers can image only parts of these organs at a source-detector distance of $150 \mathrm{~cm}$.

Another approach to predict DVH parameter changes is to perform 3D imaging during treatment in combination with repeated treatment planning. By acquisition of a cone-beam CT scan, the time between acquisition and treatment is minimal, which is preferred above the use of repeated CT scanning on a conventional CT scanner. However, this process is time-consuming, also because the breast gland has to be delineated again by the physician to account for possible breast deformations. The same is true for a 3D dose reconstruction procedure based on measured portal dose images. ${ }^{5}$ Although changes in DVH parameters can be calculated very accurately by these procedures, our method warns when DVH parameters change for the PTV using a specific tolerance level, and it is very fast because it only needs calculation of equation (7.1). Of course, 2D gamma parameters have to be determined from a gamma image, which means that a clinical 2D EPID dosimetry procedure should be 
implemented in a radiotherapy department. However, an increasing number of departments is implementing portal dosimetry in practice nowadays.

\subsection{CONCLUSIONS}

We have developed a model that predicts dose-volume histogram changes due to set-up errors during conventional breast cancer treatment using portal dose images measured by means of EPIDs. For this purpose, a simulation study has been done where set-up errors have been varied for 20 breast cancer patients and correlations were determined between different DVH parameters and sets of 2D gamma parameters for the PTV only. Gamma parameters have been calculated from gamma images which describe the agreement between PDIs with and without set-up errors. Gamma evaluation criteria of $3 \%$ and $3 \mathrm{~mm}$ were used to calculate the gamma images. The probability that a DVH parameter changes more than a specific tolerance level has been derived from a binomial logistic regression model that needs the gamma parameters as input variables. Based on the simulation study, it was found that changes in the mean PTV dose can be detected if changes in these gamma parameters are larger than $5 \%$, and that changes in $V_{90}$ and $V_{95}$ can be found if these changes are larger than $10 \%$. Real DVH parameter changes can be detected with a high accuracy, while the workload is somewhat increased due to a higher number of false positive predicted changes. The model has been verified by using clinically measured gamma images, not only for portal dose differences caused by set-up errors of patients, but also resulting from patient rotations or breast deformations. Due to these additional geometric uncertainties, the overall predictive value of our model is slightly decreased but the positive predictive values remained the same. Using our predictive model, differences in measured portal dose images can be related to changes in DVH parameters and used in a decision protocol. Compared to decision protocols based on regular portal imaging or CBCT scanning, our approach directly offers clinically relevant parameters to decide if a treatment has to be adapted or not. This work can be seen as an intermediate step between 2D transit dosimetry ${ }^{7}$ and 3D dose reconstruction. ${ }^{12}$

\section{ACKNOWLEDGMENT}

The authors would like to thank Shipeng Yu for his help in implementing the binomial logistic regression model in combination with the cross-validation-based procedure. 


\section{REFERENCES}

1 S.M.J.J.G. Nijsten, A.W.H. Minken, P. Lambin, and I.A.D. Bruinvis, "Verification of treatment parameter transfer by means of electronic portal dosimetry," Med. Phys. 31, 341-347 (2004).

2 B. Warkentin, S. Steciw, S. Rathee, and B.G. Fallone, "Dosimetric IMRT verification with a flat-panel EPID," Med. Phys. 30, 3143-3155 (2003).

3 A. Van Esch, T. Depuydt, and D.P. Huyskens, "The use of an aSi-based EPID for routine absolute dosimetric pre-treatment verification of dynamic IMRT fields," Radiother. Oncol. 71, 223-234 (2004).

4 M. van Zijtveld, M.L.P. Dirkx, H.C.J. de Boer, and B.J.M. Heijmen, "3D dose reconstruction for clinical evaluation of IMRT pretreatment verification with an EPID," Radiother. Oncol. 82, 201-207 (2007).

5 W.J.C. van Elmpt, S.M.J.J.G. Nijsten, B.J. Mijnheer, A.L.A.J. Dekker, and P. Lambin, "The next step in patient-specific QA: 3D dose verification of conformal and intensity-modulated RT based on EPID dosimetry and Monte Carlo dose calculations," Radiother. Oncol. 86, 86-92 (2008).

6 S.M.J.J.G. Nijsten, B.J. Mijnheer, A.L.A.J. Dekker, P. Lambin, and A.W.H. Minken, "Routine individualised patient dosimetry using electronic portal imaging devices," Radiother. Oncol. 83, 65-75 (2007).

7 M. Kroonwijk, K.L. Pasma, S. Quint, P.C.M. Koper, A.G. Visser, and B.J.M. Heijmen, "In vivo dosimetry for prostate cancer patients using an electronic portal imaging device (EPID); Demonstration of internal organ motion," Radiother. Oncol. 49, 125-132 (1998).

8 M. Partridge, M. Ebert, and B.M. Hesse, "IMRT verification by threedimensional dose reconstruction from portal beam measurements," Med. Phys. 29, 1847-1858 (2002).

9 L.N. McDermott, M. Wendling, J. Nijkamp, A. Mans, J.J. Sonke, B.J. Mijnheer, and M. van Herk, "3D in vivo dose verification of entire hypofractionated IMRT treatments using an EPID and cone-beam CT," Radiother. Oncol. 86, 35-42 (2008).

10 D.A. Low, W.B. Harms, S. Mutic, and J.A. Purdy, "A technique for the quantitative evaluation of dose distributions," Med. Phys. 25, 656-661 (1998).

11 D.A. Low and J.F. Dempsey, "Evaluation of the gamma dose distribution comparison method," Med. Phys. 30, 2455-2464 (2003).

12 W.J.C. van Elmpt, S.M.J.J.G. Nijsten, R.F.H. Schiffeleers, A.L.A.J. Dekker, B.J. Mijnheer, P. Lambin, and A.W.H. Minken, "A Monte Carlo based three-dimensional dose reconstruction method derived from portal dose images," Med. Phys. 33, 2426-2434 (2006).

13 W.J.C. van Elmpt, S.M.J.J.G. Nijsten, B.J. Mijnheer, and A.W.H. Minken, "Experimental verification of a portal dose prediction model," Med. Phys. 32, 2805-2818 (2005).

14 S.M.J.J.G. Nijsten, W.J.C. van Elmpt, M. Jacobs, B.J. Mijnheer, A.L.A.J. Dekker, P. Lambin, and A.W.H. Minken, "A global calibration model for a-Si EPIDs used for transit dosimetry," Med. Phys. 34, 3872-3884 (2007).

15 M. Miften, M. Wiesmeyer, S. Monthofer, and K. Krippner, "Implementation of FFT convolution and multigrid superposition models in the FOCUS RTP system," Phys. Med. Biol. 45, 817-833 (2000).

16 D. Hand, H. Mannila, and P. Smyth, "Principles of Data Mining," The MIT Press, Cambridge, Massachusetts, 2nd ed., 2001.

17 M.S. Pepe, "The Statistical Evaluation of Medical Tests for Classification and Prediction," Oxford University Press, Oxford, 1st ed., 2003.

18 C.W. Hurkmans, P. Remeijer, J.V. Lebesque, and B.J. Mijnheer, "Set-up verification using portal imaging; Review of current clinical practice," Radiother. Oncol. 58, 105-120 (2001). 
152 Chapter 7

19 C.L. Hector, S. Webb, and P.M. Evans, "The dosimetric consequences of inter-fractional patient movement on conventional and intensity modulated breast radiotherapy treatments," Radiother. Oncol. 54, 57-64 (2000).

20 L.N. McDermott, M. Wendling, J.J. Sonke, M. van Herk, and B.J. Mijnheer, "Replacing pretreatment verification with in vivo EPID dosimetry for prostate IMRT," Int. J. Radiat. Oncol., Biol., Phys. 67, 1568-1577 (2007). 


\title{
INTRODUCTION
}

\author{
POINT DOSE VERIFICATION \\ METHODS AND APPLICATION
}

TWO-DIMENSIONAL TRANSIT DOSIMETRY METHODS AND APPLICATION 

CHAPTER

General discussion and future perspectives

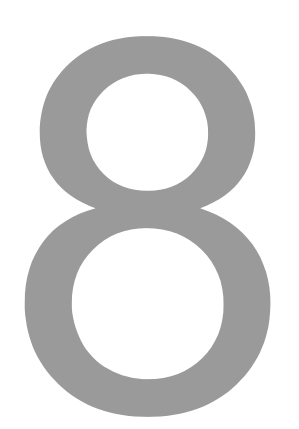





\subsection{INTRODUCTION}

The accuracy of radiotherapy should be very high to ensure a high local tumor control without severe complications due to that treatment. Mijnheer et al. ${ }^{1}$ proposed an accuracy requirement in absorbed dose delivery at the dose specification point of $3.5 \%$ (1 SD) for external beam treatments. To verify such an accurate dose delivery, different methods can be used. One method is to perform dose measurements during treatment where the measurement accuracy should be at least the proposed accuracy. When a dose measurement is compared to a predicted dose value, the accuracy of the dose prediction is equally important.

Frequently, the gamma evaluation method ${ }^{2,3}$ is used to report the agreement between dose measurements and reference dose values. This method combines a dose difference criterion and distance-to-agreement criterion to determine the agreement between two dose distributions. Commonly used gamma criteria for the evaluation of dose measurement accuracy are $3 \%$ and $3 \mathrm{~mm}^{4,5}$. Ideally, all physical methods described in this thesis should meet these criteria to apply them in clinical practice without resulting in an unacceptable number of false positive measurements. If the criteria are not met, the measurement conditions should be well-known and taken into account using decision protocols and decision trees.

The accuracy and clinical applicability of the dosimetric calibration and dose prediction models in this thesis are discussed in Sec. 8.2 and Sec. 8.3, respectively. The clinical application of point dose verification and two-dimensional transit dosimetry in our department is described in Sec. 8.4. Finally, future perspectives resulting from this thesis and current work of our research group can be found in Sec. 8.5.

\subsection{DOSIMETRIC CALIBRATION OF EPIDS}

\subsubsection{Measurement accuracy}

In this work, both video-based and $a$-Si EPIDs are dosimetrically calibrated to obtain a 2D absolute dose distribution under full-scatter conditions.

Two different video-based EPIDs have been calibrated: the iView EPID (Elekta, Crawley, United Kingdom) in chapter 2 and the intermediate-elbow Theraview-NT EPID (Cablon Medical, Leusden, The Netherlands) in chapter 3. Both EPIDs are calibrated with the dosimetric calibration model as described by Heijmen et al. ${ }^{6}$ and Pasma et al. ${ }^{7}$ Dose values could be measured within 1-2\% compared to ionization chamber measurements, under reference conditions (Chapter 2). Similar results were obtained for the SRI-100 EPID (Elekta, Crawley, United Kingdom) ${ }^{7}$.

Three different $a$-Si EPIDs were calibrated: the OptiVue 500/1000/1000 ST EPIDs (Siemens Medical Solutions, Concord, CA, USA). The dosimetric calibration that has been used is described in chapter 5 and its accuracy was determined for different treatment fields such as virtual wedge and intensity-modulated radiation therapy 
(IMRT) fields, using various phantoms. Gamma evaluation criteria of $3 \%$ and $3 \mathrm{~mm}$ were satisfied for all in-field measurement points across the EPID plane. Larger dose differences were found out-of-field and could be attributed to differences in patient scatter. Chen et $a l^{8}{ }^{8}$ described the dosimetric calibration of the same $a$-Si flat panel (Perkin-Elmer XRD 1640 AL7) as used in the OptiVue 500/1000 EPIDs with an accuracy of the calibration of $3 \%$ (2 SD) for the in-field region and local differences ranging from $-10 \%$ to $65 \%$ in the out-of-field regions. The dosimetric behavior of other commercially available $a$-Si EPIDs was studied for the aS500/1000 EPID (Varian Medical Systems, Palo Alto, CA, USA) ${ }^{9-12}$ and the $i$ View GT EPID (Elekta, Crawley, United Kingdom) ${ }^{13-15}$. The reported accuracy was very similar to our findings when no object was present in the beam but almost no attention was paid to the impact of energy spectrum changes to the EPID response when an object was positioned in the beam (transit dosimetry). In this case, an energy spectrum correction is mandatory to reduce differences in energy spectrum response of the EPID to less than 3\% as shown in chapter 5 , which is also confirmed by several other authors ${ }^{16-18}$.

Other corrections are needed for the dosimetric calibration model of the video-based EPIDs compared to the calibration model of the $a$-Si EPIDs, which is mainly due to the differences in energy dependence of both types of EPIDs. While the video-based EPIDs are relatively insensitive to changes in beam energy spectrum ${ }^{9}$, amorphous silicon EPIDs are sensitive for low energy photons ${ }^{19}$ causing changes in EPID response when the thickness of an object in the beam increases or decreases. The measurement accuracy using both dosimetric calibration models is very similar and generally better than $3 \%$ and $3 \mathrm{~mm}$.

\subsubsection{Clinical applicability}

The dosimetric calibration model for video-based EPIDs has been applied to different brands of EPIDs in the past with high accuracy (Sec. 8.2.1). The calibration model in chapter 5 has been developed for three different types of Siemens OptiVue EPIDs but is not yet applied to other $a$-Si EPIDs like the Varian aS500 and Elekta iView GT EPIDs. Because hardware specifications and hence dose characteristics ${ }^{12,13}$ are very similar to the EPIDs we used, we expect that the calibration model can be applied relatively easy to these EPIDs. The excellent agreement between ghosting effects studied for the three different brands of $a$-Si EPIDs in chapter 6 supports this statement.

Both dosimetric calibration models convert the grayscale values of an EPID image into an absolute dose distribution measured in water under full-scatter conditions. Hence, reference dose measurements in water are needed. These measurements have to be performed only once, as long as the beam characteristics of a linear accelerator do not change, and take about 4-5 hours per photon beam energy. The EPID measurements have to be repeated after each replacement or major change to EPID hardware or acquisition software and can be done in 1-2 hours per photon beam energy. In case of dosimetrically matched linear accelerators, the reference dose measurements are performed for one machine only and not all EPID measurements have to be repeated for similar EPIDs. The latter is shown in chapter 5 where good agreement was found between image lag, field size dependence and energy spectrum corrections for an 
OptiVue 500 and OptiVue 1000, which are similar in design, positioned on two identical linear accelerators.

\subsubsection{Future improvements}

The dosimetric calibration models can be improved by using Monte Carlo simulations to study the primary and scattered dose contributions in the EPID plane to modify the energy spectrum correction of the $a$-Si EPID dosimetric calibration model. This is especially important when smaller source-to-detector distances are used than the distance of $150 \mathrm{~cm}$ applied in our work. The patient scatter contribution to the EPID will increase significantly for smaller source-to-detector distances as described in chapter 4, probably decreasing the accuracy of our energy spectrum correction model.

The Siemens OptiVue EPIDs that were dosimetrically calibrated according to the method described in chapter 5 could only move in the beam direction. For EPIDs that can additionally move perpendicular to the beam direction, the calibration model has to be adapted. In this case, the flood field correction and beam profile correction have to be modified, to separate the energy dependence of the EPID without an object in the beam from the beam profile, which are now both dependent on the off-axis position of the EPID ${ }^{15}$. Monte Carlo simulations can be of help in understanding these effects and can be used to study the different effects that have to be corrected for.

\subsection{DOSE PREDICTIONS}

\subsubsection{Prediction accuracy}

Models have been developed to predict reference dose values for comparison with measured dose values.

In chapters 2 and 3, absolute dose values in the EPID plane were predicted at the geometrical center of a radiation field only, with and without a patient in the beam. The accuracy of the pre-treatment dose prediction model was investigated in chapter 2 for different types of treatment fields and the agreement between dose prediction and dose measurement in water was within $0.9 \%$ (1 SD) deviation. The accuracy has not been tested in case of irregular shaped fields which may decrease the prediction accuracy. The transit dose prediction model described in chapter 3 neglects missing tissue and uses a scatter to primary ratio ${ }^{20}$ which assumes a symmetrical distribution of inhomogeneities around the isocenter plane. Especially for treatments in the lung and head and neck region, this latter assumption is not always valid which causes a different patient scatter contribution to the EPID detector. Furthermore, the influence of field shaping by using shielding blocks or MLC on the predicted central field dose is not taken into account by the transit point dose prediction model. The same arguments are valid for the back-projection model that calculates the patient dose at 5 $\mathrm{cm}$ depth inside a patient using the measured EPID dose. Hence, due to this limited accuracy, the predicted dose will not always satisfy the $3 \%$ dose criterion. 
In chapter 4, a portal dose prediction model is investigated that calculates a 2D portal dose distribution in the EPID plane behind a patient using a pre-treatment portal dose image, the planning CT scan and pencil beam scatter kernels. Based on extensive phantom measurements, the accuracy of the model is better than $2 \%$ (mean difference). With this model, missing tissue and patient scatter contribution to the EPID plane are now modelled very accurately because a detailed patient model is available from the planning CT scan. Field shaping but also entire 2D fluence distributions are known by using a pre-treatment portal dose image. In chapter 4 , the accuracy is determined by measuring PDIs with and without objects in the beam, which is then dependent on the accuracy of the dosimetric calibration model for the EPID to obtain the PDIs. In Sec. 8.2.1, the dose measurement accuracy was discussed and showed to be better than $3 \%$ and $3 \mathrm{~mm}$ in-field.

\subsubsection{Clinical applicability}

The transit point dose prediction model described in chapter 3 is a relatively simple model that can be implemented in a department without much effort and is based on a limited set of measurements. The dose measurements in the huge water tank take 1-2 hours per photon beam energy. They can be used to accurately calculate central field dose values in case of conventional multi segment treatment techniques but this set of measurements is too limited for more elaborate techniques like IMRT. The same is true for the pre-treatment point dose prediction model in chapter 2.

The 2D transit portal dose prediction model described in chapter 4 is accurate for dose predictions in case of IMRT fields but its implementation is more complex. Furthermore, a larger measurement set is needed to derive the pencil beam scatter kernels, which can be obtained in 3-4 hours per photon beam energy. For dosimetrically matched linear accelerators, these measurements have to be performed for one machine only.

A pre-treatment portal dose image is used by the 2D transit dose prediction model, which was measured with an EPID in chapter 4 to determine the accuracy of the prediction model. In clinical practice, it is important to have dose predictions that are fully independent of portal dose measurements, avoiding false negative dose differences. This requires a prediction of the pre-treatment PDI and therefore, a 2D pre-treatment portal dose prediction model is implemented and used for this purpose during clinical routine.

Both the 2D pre-treatment and transit portal dose prediction models can replace the point dose prediction models described in chapters 2 and 3. The 2D models are more accurate and wider applicable but do need more input parameters. 


\subsection{CLINICAL APPLICATION}

\subsubsection{Point dose verification}

In chapter 3, clinical results are shown from our routine pre-treatment verification and individual patient dosimetry programme. Camera-based EPIDs were dosimetrically calibrated and measured central field dose values were compared to predicted point dose values. Dose comparisons were performed in the EPID plane and at a depth of 5 $\mathrm{cm}$ inside the patient. Action levels were used to decide if additional actions were necessary prior to the next treatment or if dose differences were within the acceptable limits.

Using the point dose verification procedures in clinical routine, several dose delivery errors were found, which means that the dose delivered to a patient would change more than 3-5\% compared to the prescribed dose, if no action was undertaken.

For about $1 \%$ of all pre-treatment sessions, significant dose differences were detected caused by transfer and machine errors. Most of these errors were caused by the fact that no electronic transfer was present between treatment planning system (TPS) and treatment equipment. Fiorino et al. ${ }^{21}$ reported a rate of $1.5 \%$ dose differences per patient larger than $5 \%$ or more in the delivered dose to the PTV caused by errors made during treatment planning and data transfer. By implementing an electronic transfer in 2005, the transfer errors were eliminated. However, new transfer errors were introduced because treatment parameters could still be accidently changed in the record-and-verify system. Using the pre-treatment dose verification procedures, these errors have been found and can trigger radiation therapy technicians to look again at the actual treatment parameter values.

For treatments in the pelvic region, transit dose differences were often found due to the presence of gas pockets in the rectum which could lead to replanning using a new CT scan or implementing a specialized diet for this group of patients. The occurrence of gas pockets during portal dosimetry has also been reported by several other authors $^{22-24}$. For breast treatments, set-up errors up to $1.5 \mathrm{~cm}$ were detected in the lateral-medial direction causing large differences in the central field dose. In a review, Hurkmans et al. ${ }^{25}$ also reported large standard deviations (1 SD) of systematic and random set-up errors for breast treatments, ranging from 1.0-4.7 $\mathrm{mm}$ and 1.7-14.4 $\mathrm{mm}$, respectively. For breast, lung and head and neck region, transit dose measurements were often very sensitive to set-up errors and organ motion.

Pre-treatment and transit portal dose measurements can be compared to entrance and exit point dose measurements ${ }^{21,26-27}$, respectively, using traditional point detectors (Sec. 1.3). The observed dose differences and reported error sources are similar but it should be noted that the EPID measurements are not sensitive to changes in source-to-skin distance (SSD). This is because EPIDs are located at a relatively large distance to the patient to minimize the contribution of patient scatter in the EPID plane, which makes the measurement insensitive to (small) changes of patient position in the beam direction. However, the SSD difference itself can be detected during normal portal imaging procedures and are therefore uncommon. 
The point dose verification procedures based on EPID measurements are very fast and do not require additional machine time for individual transit dosimetry measurements. The analysis of the EPID images took less than $20 \mathrm{~s}$ per image if dose differences did not exceed prescribed action levels.

The number of false positive dose delivery errors was equal to or larger than the number of true positive dose delivery errors (Fig. 3.4). These false positive dose delivery errors limit the use of smaller action levels and hence the detection of small dose delivery errors. Consequently, the verification procedures should be optimized to minimize all errors besides the true dose delivery errors. This can be done by decreasing the number of acquisition errors (Sec. 3.4.1), user errors (Sec. 3.4.2) and implementation errors (Sec. 3.4.3). Furthermore, procedures have to be improved, which will decrease the number of errors due to procedure limitations (Sec. 3.4.4).

Acquisition errors are dependent on the equipment that is used for treatment and verification. Nowadays, hardware and acquisition software of EPIDs are more and more integrated in a linear accelerator, decreasing the number of acquisition errors. User errors are minimized by making all processes automated. Many user errors during clinical point dose verification were caused by the manual determination of the individual patient parameters used for the back-projection of the measured transit dose. The technical infrastructure for handling and analysis of portal images that was implemented in our department to eliminate user errors, is discussed in the Appendix. Implementation errors in (in-house developed) software should be decreased by using well-established guidelines for development, commissioning and verification of software. By these improvements, the number of false positive dose delivery errors can already significantly be reduced (Fig. 3.4). However, more than $50 \%$ of all false positive errors for head and neck patients were caused by procedure limitations. By changing from point dose verification to two-dimensional transit dosimetry, the number of errors due to procedure limitations has been decreased and the positioning problems of the dose verification point as discussed earlier in this section are avoided.

\subsubsection{Two-dimensional transit dosimetry}

Using the 2D transit portal dose prediction model described in chapter 4, a reference dose distribution in the EPID plane can be calculated and compared to a measured portal dose image based on the gamma evaluation method ${ }^{2,3}$. This procedure has been implemented clinically in our department since 2006 and is applied to all patients treated with curative intent (for details see the Appendix). By using the 2D portal dose prediction model, a number of procedure limitations encountered during point dose verification is solved (Secs. 3.4.4 and 8.3.1). Furthermore, transit dose values are now verified in two dimensions making the dose verification results less sensitive to set-up errors and organ motion. In Fig. 7.6, gamma images are shown for a breast cancer treatment, calculated with gamma criteria of $3 \%$ and $3 \mathrm{~mm}$. Locally varying gamma value distributions are observed which are caused by a different arm position at the cranial field edge and (small) deformations of the breast during treatment. With point 
dose verification based on the central field dose value only, it is impossible to identify these error sources.

Two-dimensional transit dosimetry is more complex and calculations are more time consuming. Portal dose predictions take up to 10-15 minutes per IMRT treatment plan while portal dose conversions can be performed within 10-20 seconds per grayscale EPID image. However, all portal dose predictions and portal dose conversions are automated in our department which means that they do not need any user intervention (for details see the Appendix). Portal dose predictions are done immediately after the transfer of treatment parameters to the record-and-verify (R\&V) system which is usually at least 1 day before the first treatment. Portal dose conversion is performed per EPID image and hence most portal dose images and gamma images are already calculated after the acquisition of the last field of a treatment session.

In case of point dose verification, a dose comparison was also performed at a depth of $5 \mathrm{~cm}$ inside the patient. The latter dose verification allows for detecting errors in the treatment planning software because the reference dose is directly determined with the treatment planning system. In case of 2D transit dosimetry, measured dose values are compared to predicted dose values in the EPID plane only, which are obtained by an independent prediction based on the treatment parameters of the TPS. This means that errors in the treatment planning software (e.g. monitor unit calculation) can not be detected using this procedure.

Another procedure that is implemented clinically is 2D pre-treatment portal dose verification. Using this procedure, open field dose distributions in the EPID plane are predicted (Sec. 8.3.2) and compared to measured portal dose images acquired pre-treatment (Chapter 5). Gamma value distributions are analyzed to verify the delivery by the treatment machine in the absence of patient related delivery errors, because no object is in the beam now. The procedure is especially powerfull as pre-treatment quality assurance tool of individual IMRT treatments and it is entirely performed by radiation therapy technicians in our department. For transit dose measurements with $a$-Si EPIDs, our energy spectrum correction model uses the measured pre-treatment EPID images too, but now to obtain measured transmission values (Chapter 5). The additional "treatment time" on the treatment machine per treatment plan is 5-10 minutes. Dose delivery errors related to the treatment beam itself can also be found by using the $2 \mathrm{D}$ transit portal dose measurements but can be masked by patient related delivery errors like set-up errors and organ motion. However, it is still possible to leave the $2 \mathrm{D}$ pre-treatment portal dose verification out of the clinical routine, because the energy spectrum correction model can also use predicted pre-treatment EPID images to obtain transmission values. 


\subsection{FUTURE PERSPECTIVES}

\subsubsection{Decision protocols for 2D transit dosimetry}

Two-dimensional transit dosimetry is current clinical practice in our department for all patients treated with curative intent. Gamma images are calculated using $5 \%$ as local dose-difference criterion and $5 \mathrm{~mm}$ as distance-to-agreement criterion. Furthermore, gamma images fail the criteria if the percentage of pixels with a gamma value larger than 1 is more than $10 \%$ within the treatment field plus a $1 \mathrm{~cm}$ margin to the field edges. These clinical criteria are rather large to limit the workload for radiation therapy technicians and medical physicists. Because the prediction and conversion models are generally better than $3 \%$ and $3 \mathrm{~mm}$ (Sec. 8.2.1 and Sec. 8.3.1), the measured dose differences are predominantly true positive and are caused by set-up errors, organ motion, deformations and problems in patient fixation. More frequent treatment imaging and adaptation using image-guidance procedures will decrease the dose differences and finally the clinical gamma criteria. However, because a gamma image of every single beam is examined, 2D transit dosimetry is very sensitive to geometric and dosimetric uncertainties during treatment which do not always influence the 3D delivered dose distribution inside a patient. A future improvement can therefore be obtained by weighting 2D gamma parameter values for different beams of a treatment session before judging them by an action level. The weighting can be done by using the number of monitor units and/or the radiological thickness projected on the EPID plane. The radiological thickness can be calculated for the CTV or PTV, but also for organs at risk using the equivalent homogeneous phantom concept described by Pasma et al. ${ }^{28}$ and in chapter 4.

Another improvement is to make a distinction between systematic and random dose differences. This can be done by averaging transit gamma images from multiple treatment sessions before calculating the weighted gamma parameters. This approach is very similar to the use of off-line correction set-up protocols where the systematic set-up error of a patient is determined and corrected based on multiple set-up measurements during different treatment sessions. Hence, the workload of 2D transit dosimetry can be decreased because not every single gamma image has to be examined anymore and random dose differences are averaged out. Off-line transit dose decision protocols using such an averaging procedure can be implemented based on multiple 2D gamma parameters ${ }^{29}$ and for different patient structures based on the treatment site. Using the predictive model from chapter 7 , it is also possible to develop an off-line decision protocol based on the number of treatment sessions with a clinically relevant change in a DVH parameter.

\subsubsection{D dose reconstruction}

Last years, physical models have been investigated and implemented to reconstruct the 3D dose distribution inside a phantom or patient based on electronic portal imaging $^{30}$. These methods are based on EPID measurements performed pre-treatment $^{31-35}$ and during treatment ${ }^{24,36-38}$, which are both investigated and 

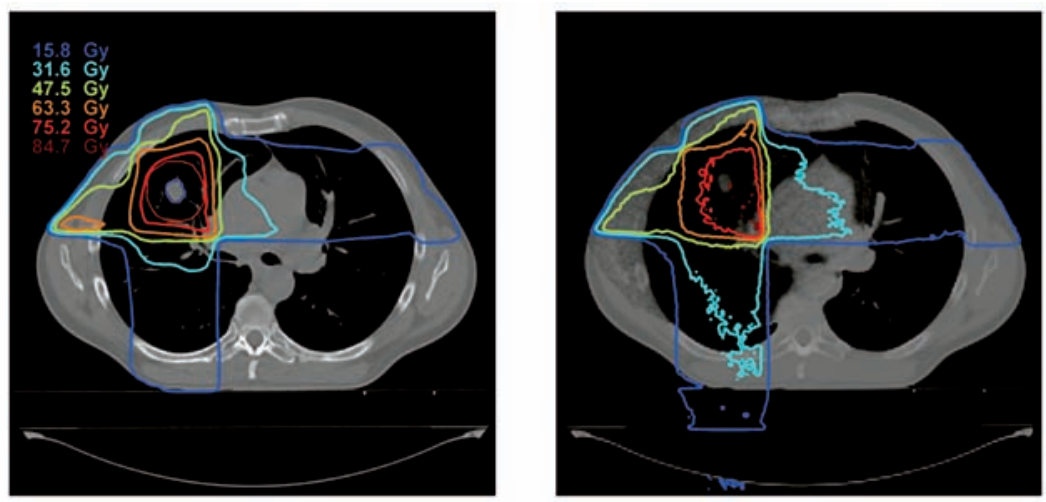

(a)

(b)
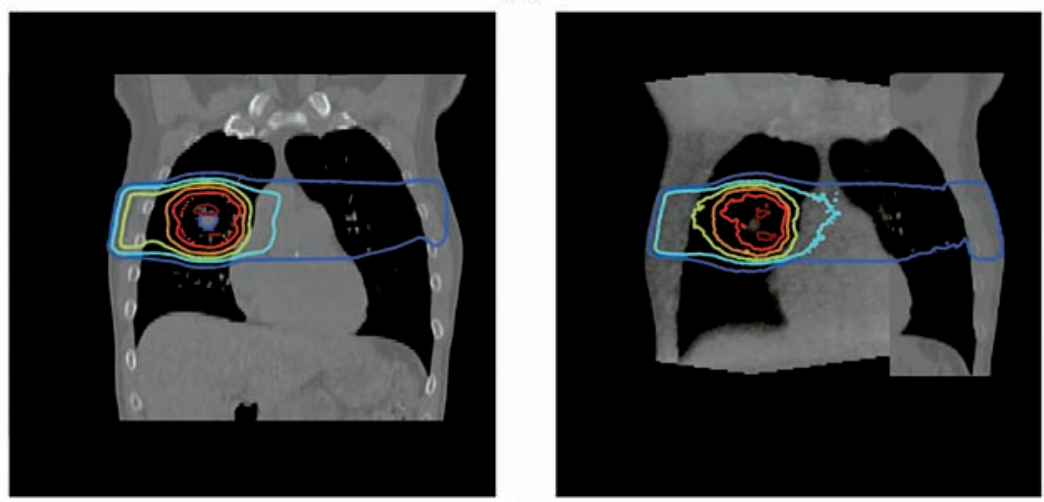

(c)

(d)

Figure 8.1: An example of 3D dose reconstruction during the treatment of a lung cancer patient. The dose distribution from the treatment planning system based on the planning CT scan is shown in (a) an axial view and (c) a coronal view of the patient. Based on measured transit portal dose images during one treatment session, a 3D dose reconstruction has been performed using Monte Carlo simulations ${ }^{37}$. For this reconstruction, a megavoltage cone-beam CT scan is acquired just prior to the treatment session. In (b) and (d), the reconstructed dose distribution is shown using the same axial and coronal views as in (a) and (c). Because the field-of-view of the megavoltage cone-beam CT scan is limited, the missing tissue is taken from the planning CT scan.

implemented in our department. Dose reconstructions are based on 2D portal dose images obtained with the dosimetric calibration model for a-Si EPIDs (Chapters 5-6). A planning CT scan or a megavoltage cone-beam CT $\operatorname{scan}^{39}$ can be used for 3D dose reconstruction using Monte Carlo simulations where the megavoltage cone-beam CT scan has to be corrected for cupping artifacts ${ }^{40}$. In case of $3 D$ in vivo dosimetry, the 2D transit portal dose prediction model of chapter 4 is iteratively applied to correct for patient scattered radiation. In Fig. 8.1., an example of 3D dose reconstruction during the treatment of a lung cancer patient is shown. 
Three-dimensional dose reconstruction based on 2D transit portal dose images can yield the ultimate verification of the dose delivery in the actual patient anatomy for a particular treatment session. Furthermore, it opens possibilities for adaptive radiotherapy and dose-guided radiation therapy strategies, taking into account the 3D delivered dose distribution during multiple previous treatment sessions. However, 3D dose reconstruction is not yet applied in our department on a large scale in clinical practice. A first application can be based on the current routine use of 2D transit dosimetry where $3 \mathrm{D}$ dose reconstruction will be performed when $2 \mathrm{D}$ transit portal dose differences can not be explained and need further investigation.

\subsubsection{Computer Aided Theragnostics and Voxel Dose Probability}

Computer Aided Theragnostics (CAT) is a technique where machine learning is applied to one or multiple databases with large amounts of patient data to predict the probability of treatment outcome, survival or complications ${ }^{41}$. The types of patient data are diverse, like information from medical and biological examination, treatment planning, treatment verification and patient follow-up after treatment. Results from treatment verification using dose measurements are stored into the CAT databases, including predicted and measured point dose values and 2D portal dose images, 2D gamma images and (in future) also the reconstructed delivered 3D dose distributions. Based on these dosimetric data, qualitative and quantitative predictions are performed incorporating the dosimetric uncertainties during radiotherapy treatment. Consequently, more accurate dose-effect curves for local tumor control and normal tissue damage per patient will be obtained.

Using probabilistic treatment planning ${ }^{42}$, uncertainties during treatment are included in a treatment planning procedure to optimize the probability that a specific dose in a voxel will be applied during treatment. This probability is also called Voxel Dose Probability and will be influenced by geometric but also dosimetric uncertainties. Dose-guided radiation therapy using $2 \mathrm{D}$ dose measurements and $3 \mathrm{D}$ dose reconstruction is a crucial element to track the dose given to each voxel, sharpening the Voxel Dose Probability even more during a treatment, until the last treatment session where the true delivered dose to a voxel is known. This is current work of our research group which has just started but also shows the huge potential of performing portal dosimetry during radiotherapy. 


\section{REFERENCES}

1 B.J. Mijnheer, J.J. Battermann, and A. Wambersie, "What degree of accuracy is required and can be achieved in photon and neutron therapy?" Radiother. Oncol. 8, 237-252 (1987).

2 D.A. Low, W.B. Harms, S. Mutic, and J.A. Purdy, "A technique for the quantitative evaluation of dose distributions," Med. Phys. 25, 656-661 (1998).

3 D.A. Low and J.F. Dempsey, "Evaluation of the gamma dose distribution comparison method," Med. Phys. 30, 2455-2464 (2003).

4 P. Sandilos, A. Angelopoulos, P. Baras, K. Dardoufas, P. Karaiskos, P. Kipouros, M. Kozicki, J.M. Rosiak, L. Sakelliou, I. Seimenis, and L. Vlahos, "Dose verification in clinical IMRT prostate incidents," Int. J. Radiat. Oncol. Biol. Phys. 59, 1540-1547 (2004).

5 M. Oldham, H. Sakhalkar, P. Guo, and J. Adamovics, "An investigation of the accuracy of an IMRT dose distribution using two- and three-dimensional dosimetry techniques," Med. Phys. 35, 2072-2080 (2008).

6 B.J.M. Heijmen, K.L. Pasma, M. Kroonwijk, V.G.M. Althof, J.C.J. de Boer, A.G. Visser, and H. Huizenga, "Portal dose measurement in radiotherapy using an electronic portal imaging device (EPID)," Phys. Med. Biol. 40, 1943-1955 (1995).

7 K.L. Pasma, M. Kroonwijk, J.C.J. de Boer, A.G. Visser, and B.J.M. Heijmen, "Accurate portal dose measurement with a fluoroscopic electronic portal imaging device (EPID) for open and wedged beams and dynamic multileaf collimation," Phys. Med. Biol. 43, 2047-2060 (1998).

8 J. Chen, C.F. Chuang, O. Morin, M. Aubin, and J. Pouliot, "Calibration of an amorphous-silicon flat panel portal imager for exit-beam dosimetry," Med. Phys. 33, 584-594 (2006).

9 E.E. Grein, R. Lee, and K. Luchka, "An investigation of a new amorphous silicon electronic portal imaging device for transit dosimetry," Med. Phys. 29, 2262-2268 (2002).

10 P.B. Greer and C.C. Popescu, "Dosimetric properties of an amorphous silicon electronic portal imaging device for verification of dynamic intensity modulated radiation therapy," Med. Phys. 30, 1618-1627 (2003).

11 B. Warkentin, S. Steciw, S. Rathee, and B.G. Fallone, "Dosimetric IMRT verification with a flat-panel EPID," Med. Phys. 30, 3143-3155 (2003).

12 A. Van Esch, T. Depuydt, and D.P. Huyskens, "The use of an aSi-based EPID for routine absolute dosimetric pre-treatment verification of dynamic IMRT fields," Radiother. Oncol. 71, 223-234 (2004).

13 L.N. McDermott, R.J.W. Louwe, J.J. Sonke, M.B. van Herk, and B.J. Mijnheer, "Dose-response and ghosting effects of an amorphous silicon electronic portal imaging device," Med. Phys. 31, 285-295 (2004).

14 R.J.W. Louwe, L.N. McDermott, J.J. Sonke, R. Tielenburg, M. Wendling, M.B. van Herk, and B.J. Mijnheer, "The long-term stability of amorphous silicon flat panel imaging devices for dosimetry purposes," Med. Phys. 31, 2989-2995 (2004).

15 P.B. Greer, "Correction of pixel sensitivity variation and off-axis response for amorphous silicon EPID dosimetry," Med. Phys. 32, 3558-3568 (2005).

16 B.M.C. McCurdy, K. Luchka, and S. Pistorius, "Dosimetric investigation and portal dose image prediction using an amorphous silicon electronic portal imaging device," Med. Phys. 28, 911-924 (2001).

17 C. Kirkby and R. Sloboda, "Consequences of the spectral response of an a-Si EPID and implications for dosimetric calibration," Med. Phys. 32, 2649-2658 (2005). 
18 W. Li, J.V. Siebers, and J.A. Moore, "Using fluence separation to account for energy spectra dependence in computing dosimetric a-Si EPID images for IMRT fields," Med. Phys. 33, 4468-4480 (2006).

19 C. Yeboah and S. Pistorius, "Monte Carlo studies of the exit photon spectra and dose to a metal/phosphor portal imaging screen," Med. Phys. 27, 330-339 (2000).

20 W. Swindell and P.M. Evans, "Scattered radiation in portal images: a Monte Carlo simulation and a simple physical model," Med. Phys. 23, 63-73 (1996).

21 C. Fiorino, D. Corletto, P. Mangili, S. Broggi, A. Bonini, G.M. Cattaneo, R. Parisi, A. Rosso, P. Signorotto, E. Villa, and R. Calandrino, "Quality assurance by systematic in vivo dosimetry: results on a large cohort of patients," Radiother. Oncol. 56, 85-95 (2000).

22 M. Kroonwijk, K.L. Pasma, S. Quint, P.C.M. Koper, A.G. Visser, and B.J.M. Heijmen, "In vivo dosimetry for prostate cancer patients using an electronic portal imaging device (EPID); demonstration of internal organ motion," Radiother. Oncol. 49, 125-132 (1998).

23 K.L. Pasma, M. Kroonwijk, S. Quint, A.G. Visser, and B.J.M. Heijmen, "Transit dosimetry with an electronic portal imaging device (EPID) for 115 prostate cancer patients," Int. J. Radiat. Oncol. Biol. Phys. 45, 1297-1303 (1999).

24 L.N. McDermott, M. Wendling, J. Nijkamp, A. Mans, J.J. Sonke, B.J. Mijnheer, and M. van Herk, "3D in vivo dose verification of entire hypo-fractionated IMRT treatments using an EPID and cone-beam CT," Radiother. Oncol. 86, 35-42 (2008).

25 C.W. Hurkmans, P. Remeijer, J.V. Lebesque, and B.J. Mijnheer, "Set-up verification using portal imaging; review of current clinical practice," Radiother. Oncol. 58, 105-120 (2001).

26 M. Essers, R. Keus, J.H. Lanson, and B.J. Mijnheer, "Dosimetric control of conformal treatment of parotid gland tumours," Radiother. Oncol. 32, 154-162 (1994).

27 J.H. Lanson, M. Essers, G.J. Meijer, A.W. Minken, G.J. Uiterwaal, and B.J. Mijnheer, "In vivo dosimetry during conformal radiotherapy: requirements for and findings of a routine procedure," Radiother. Oncol. 52, 51-59 (1999).

28 K.L. Pasma, B.J.M. Heijmen, M. Kroonwijk, and A.G. Visser, "Portal dose image (PDI) prediction for dosimetric treatment verification in radiotherapy. I. An algorithm for open beams," Med. Phys. 25, 830-840 (1998).

29 L.N. McDermott, M. Wendling, J.J. Sonke, M. van Herk, and B.J. Mijnheer, "Replacing pretreatment verification with in vivo EPID dosimetry for prostate IMRT," Int. J. Radiat. Oncol. Biol. Phys. 67, 1568-1577 (2007).

30 W.J.C. van Elmpt, L.N. McDermott, S.M.J.J.G. Nijsten, M. Wendling, P. Lambin, and B.J. Mijnheer, "A literature review of electronic portal imaging for radiotherapy dosimetry," Radiother. Oncol. 88, 289-309 (2008).

31 W.D. Renner, K. Norton, and T. Holmes, "A method for deconvolution of integrated electronic portal images to obtain incident fluence for dose reconstruction," J. Appl. Clin. Med. Phys. 6, 22-39 (2005).

32 M. van Zijtveld, M.L.P. Dirkx, H.C.J. de Boer, and B.J.M. Heijmen, "3D dose reconstruction for clinical evaluation of IMRT pretreatment verification with an EPID," Radiother. Oncol. 82, 201-207 (2007).

33 W.J.C. van Elmpt, S.M.J.J.G. Nijsten, R.F.H. Schiffeleers, A.L.A.J. Dekker, B.J. Mijnheer, P. Lambin, and A.W.H. Minken, "A Monte Carlo based three-dimensional dose reconstruction method derived from portal dose images," Med. Phys. 33, 2426-2434 (2006).

34 W.J.C. van Elmpt, S.M.J.J.G. Nijsten, A.L.A.J. Dekker, B.J. Mijnheer, and P. Lambin, "Treatment verification in the presence of inhomogeneities using EPID-based three-dimensional dose reconstruction," Med. Phys. 34, 2816-2826 (2007).

35 W.J.C. van Elmpt, S.M.J.J.G. Nijsten, B.J. Mijnheer, A.L.A.J. Dekker, and P. Lambin, "The next step in patient-specific QA: 3D dose verification of conformal and intensity-modulated RT 
based on EPID dosimetry and Monte Carlo dose calculations," Radiother. Oncol. 86, 86-92 (2008).

36 V.N. Hansen, P.M. Evans, and W. Swindell, "The application of transit dosimetry to precision radiotherapy," Med. Phys. 23, 713-721 (1996).

37 W.J.C. van Elmpt, S.M.J.J.G. Nijsten, S.F. Petit, B.J. Mijnheer, P. Lambin, and A.L.A.J. Dekker, "3D in vivo dosimetry using megavoltage cone-beam CT and EPID dosimetry," Int. J. Radiat. Oncol. Biol. Phys. 73, 1580-1587 (2009).

38 J. Chen, O. Morin, M. Aubin, M.K. Bucci, C.F. Chuang, and J. Pouliot, "Dose-guided radiation therapy with megavoltage cone-beam CT," Br. J. Radiol. 79 Spec. No. 1, S87-98 (2006).

39 O. Morin, A. Gillis, J. Chen, M. Aubin, M.K. Bucci, M. Roach, 3rd, and J. Pouliot, "Megavoltage cone-beam CT: system description and clinical applications," Med. Dosim. 31, 51-61 (2006).

40 S.F. Petit, W.J.C. van Elmpt, S.M.J.J.G. Nijsten, P. Lambin, and A.L.A.J. Dekker, "Calibration of megavoltage cone-beam CT for radiotherapy dose calculations: correction of cupping artifacts and conversion of CT numbers to electron density," Med. Phys. 35, 849-865 (2008).

41 C. Dehing-Oberije, D. De Ruysscher, H. van der Weide, M. Hochstenbag, G. Bootsma, W. Geraedts, C. Pitz, J. Simons, J. Teule, A. Rahmy, P. Thimister, H. Steck, and P. Lambin, "Tumor volume combined with number of positive lymph node stations is a more important prognostic factor than TNM stage for survival of non-small-cell lung cancer patients treated with (chemo)radiotherapy," Int. J. Radiat. Oncol. Biol. Phys. 70, 1039-1044 (2008).

42 C. Baum, M. Alber, M. Birkner, and F. Nusslin, "Robust treatment planning for intensity modulated radiotherapy of prostate cancer based on coverage probabilities," Radiother. Oncol. 78, 27-35 (2006). 



\section{APPENDIX}

MAASTRO implementation of large-scale

2D portal dosimetry 



\section{A.1 INTRODUCTION}

Two-dimensional transit dosimetry is applied to all patients treated with a curative intent in MAASTRO CLINIC. Besides 2D transit dosimetry, 2D pre-treatment portal dose verification is also routinely performed prior to treatment. Portal dose images are obtained by converting grayscale EPID images into portal dose images, using the dosimetric calibration model described in chapters 5-6. These measured portal dose images are compared to reference portal dose images obtained from 2D pre-treatment and transit portal dose predictions (Chapter 4), using the gamma evaluation method ${ }^{1,2}$. The gamma evaluation results are reviewed by radiation therapy technicians and medical physicists.

In this Appendix, the technical infrastructure is discussed that allows the current large-scale application of 2D portal dosimetry in clinical practice. In Sec. A.2, the overall infrastructure is presented that is used for handling and analyzing most of the information that is available to our department. In Sec. A.3, the clinical implementation and workflow of 2D portal dosimetry is discussed, including some statistics for the image handling process and its clinical reliability.

\section{A.2 TECHNICAL INFRASTRUCTURE}

In Fig. A.1, a schematic overview is shown of the technical infrastructure that is used by MAASTRO CLINIC to process and store different types of information. This information can originate from 2D, 3D or 4D imaging, treatment planning, image-guided and dose-guided radiation therapy. Most information is generated by our department, but information may also originate from an external, referring hospital (e.g. computed tomography or magnetic resonance imaging scans).

All information is collected and stored in a central Picture Archiving and Communication System (PACS) ${ }^{3-5}$ which means that all information should be present in or converted to a standard format, which is in our case the DICOM (Digital Imaging and Communications in Medicine) format ${ }^{6,7}$. To get all DICOM objects of a patient grouped together in the central PACS database, naming conventions and patient IDs should be normalized. Therefore, a connection is set-up with our Electronic Medical Record (EMR) which is the leading source of personal medical information of a patient. All DICOM objects that enter the central PACS database are forced to conform to the EMR by using an in-house developed software package called DIGITrans. Besides ensuring consistency between EMR and PACS, this software also validates every DICOM object, e.g.:

- Is it allowed to store a DICOM object in the central PACS database?

- Are the treatment parameter values such, that the treatment plan can be delivered with the treatment equipment?

- Are transit portal dose measurements possible at a source-to-detector distance of $150 \mathrm{~cm}$ (Sec. A.3.3)? 


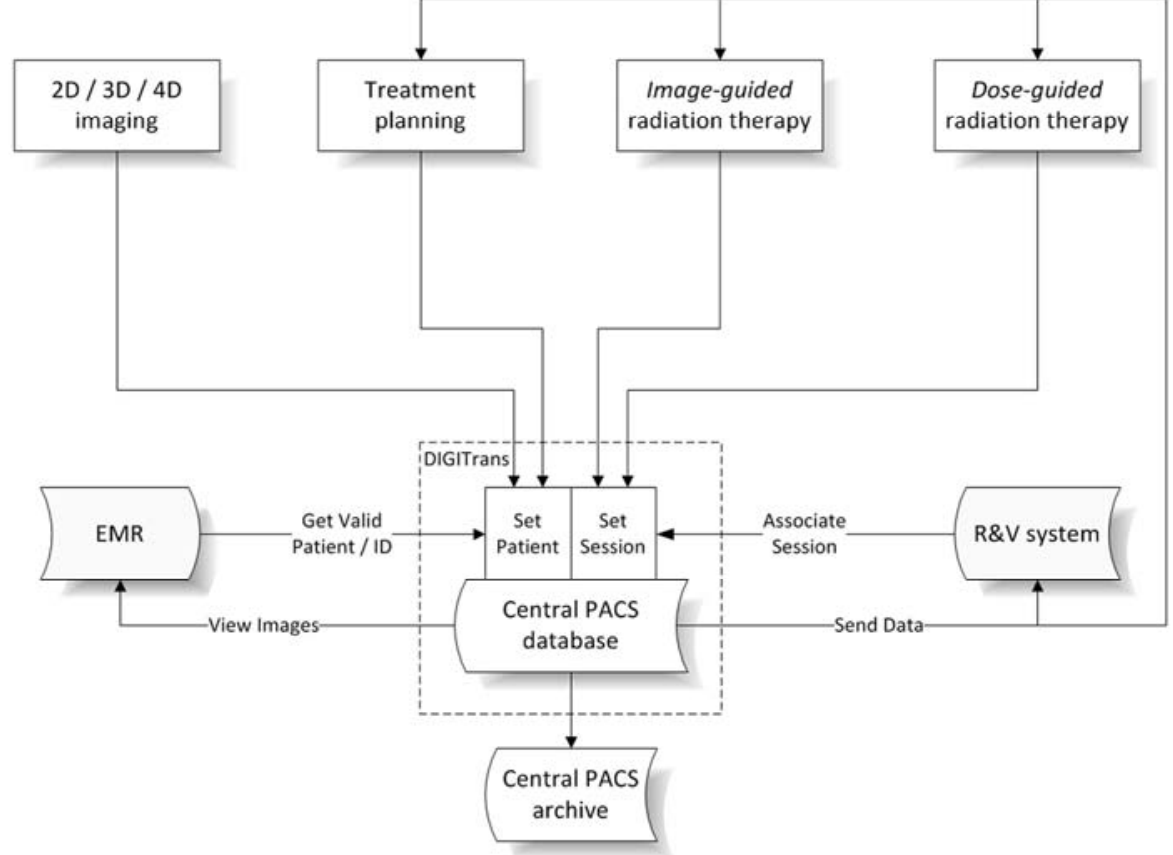

Figure A.1: Overview of the technical infrastructure for handling and storing information.

DIGITrans is a stand-alone application with multiple event-based interconnected DICOM services. For instance, one DICOM service associates DICOM objects acquired during treatment verification with a specific treatment session. In this case, fractionation information is retrieved from the record-and-verify (R\&V) system. After patient treatment, data in the central PACS can be archived by moving it to another PACS which serves as the central archive of our department.

\section{A.3 CLINICAL IMPLEMENTATION OF 2D PORTAL DOSIMETRY}

\section{A.3.1 DICOM services}

The most important building blocks of two-dimensional portal dosimetry are a number of DICOM services which interact on the central PACS database as described in Sec. A.2. These DICOM services are schematically shown in Fig. A.2 and perform the pre-treatment dose prediction, the transit dose prediction, the EPI dose conversion and the gamma calculation.

The pre-treatment dose prediction starts when the corresponding DICOM service retrieves a treatment plan (RTPLAN) from DIGITrans and calculates a pre-treatment portal dose image (RTDOSE PRED P) per beam which is stored in the central PACS. 


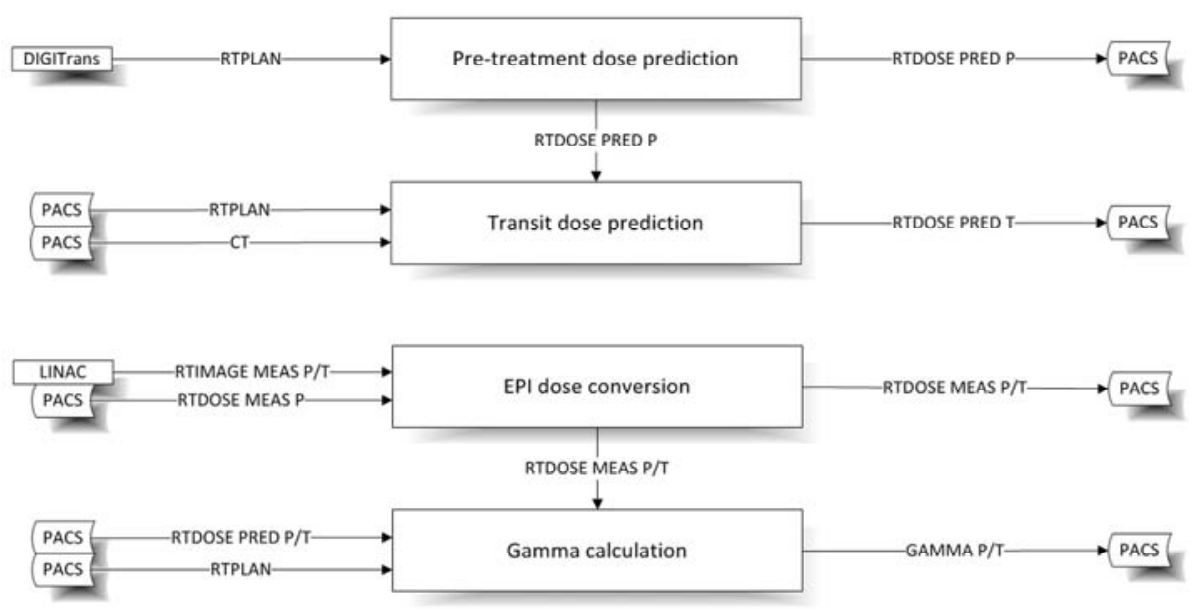

Figure A.2: Overview of the DICOM services which are the most important elements of the $2 D$ portal dosimetry workflow. All portal dose images and gamma images are stored in the central PACS database, also shown in Fig. A.1 (P: pre-treatment images, T: transit dosimetry images).

The transit dose prediction is triggered automatically by the pre-treatment dose prediction after the pre-treatment PDI is calculated. Secondary input DICOM objects of the DICOM service that predicts a transit PDI (RTDOSE PRED T) are the treatment plan (RTPLAN) and the planning CT scan of a patient (CT), which are automatically extracted from the central PACS.

Both portal dose predictions can be done within 10-15 minutes per IMRT treatment plan depending on the number of segments per beam. For non-IMRT treatment plans, the calculation time per beam is about 5-7 $\mathrm{s}$ and 60-70 $\mathrm{s}$ for the pre-treatment and transit dose prediction, respectively.

The EPI dose conversion is started after the acquisition of the first portal image has been performed and the image (RTIMAGE MEAS P/T) is auto-forwarded by the linear accelerator console to this DICOM service. This means that the first dose conversions (RTDOSE MEAS P/T) are already finished during the course of a treatment session. In case of transit dosimetry, transmission values are calculated based on measured pre-treatment EPID images. The DICOM service therefore uses pre-treatment portal dose images (RTDOSE MEAS P) as secondary input. EPI dose conversions are done within 10-20 s per portal image.

The gamma calculation is automatically triggered by the EPI dose conversion. Besides a measured portal dose image, the corresponding predicted portal dose image (RTDOSE PRED $P / T$ ) and the treatment plan (RTPLAN) is retrieved from the central PACS database as secondary input. The treatment plan is used to calculate a treatment field mask which is applied during the automatic gamma image analysis. Finally, the gamma image and the results of the gamma analysis are stored in the central PACS in DICOM RTDOSE format. The maximum calculation time of a gamma image is 15-20 s. 


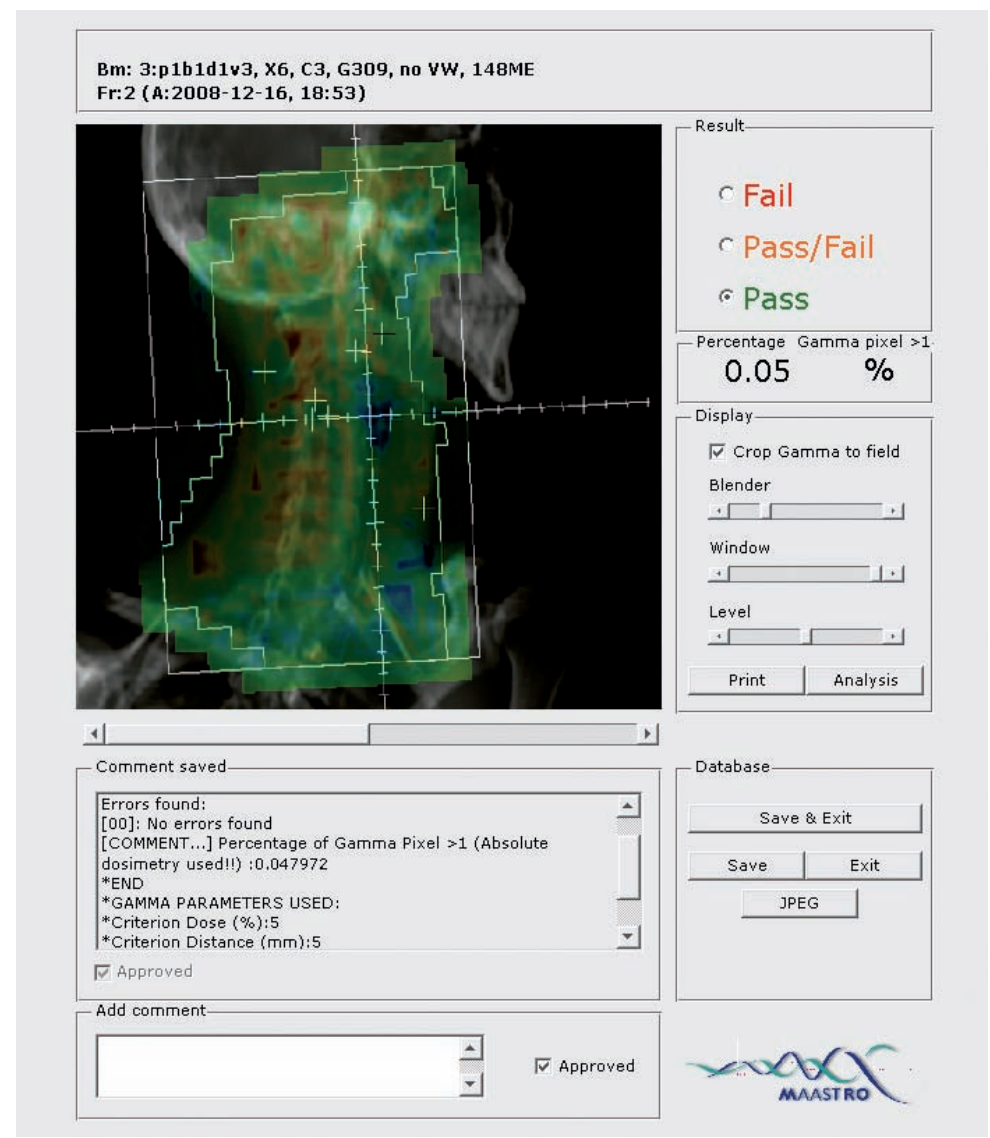

Figure A.3: Screenshot of the gamma image evaluation screen in EPICore, showing a transit gamma image acquired during an IMRT treatment in the head and neck region. Besides beam characteristics, the used gamma criteria and the gamma analysis results are shown. For this treatment field, the gamma image satisfies the gamma criteria of 5\% and $5 \mathrm{~mm}$ because only $0.05 \%$ of the pixels within the treatment field plus a $1 \mathrm{~cm}$ margin to the field edges have a gamma value larger than 1 . The tolerance level for the percentage of pixels is $10 \%$ in case of transit dosimetry.

\section{A.3.2 EPICore application}

Because all calculations for large-scale 2D portal dosimetry are automated and the results are stored centrally, the only user intervention is the examination of the PDIs and gamma images from the central PACS database. For this purpose, an application called EPICore is developed in-house on top of the PACS system to view, evaluate and annotate the portal dosimetry results. In Fig. A.3, the gamma image evaluation screen in EPICore is shown for an IMRT treatment in the head and neck region. 


\section{A.3.3 Current limitations}

In clinical practice and in this work, a fixed source-to-detector distance of $150 \mathrm{~cm}$ is used which means that not every field can be imaged because the electronics of the $a$-Si EPIDs would otherwise be damaged by radiation exposure. This problem has been solved in case of pre-treatment dose verification by duplicating all treatment fields in our R\&V system and clipping them to the maximum field-of-view of the EPIDs at 150 $\mathrm{cm}$. This is done by DIGITrans when the treatment plan is transferred. Entrance dose measurements with MOSFETs are performed during treatment if fields can not be imaged due to the limited field-of-view, or in case of possible collisions between EPID and treatment couch.

\section{A.3.4 Statistics and clinical reliability}

In our department, about 1200 patients are treated each year with a curative intent (based on statistics for 2008). Using 2D portal dosimetry, approximately 7500 and 21000 treatment fields are imaged pre-treatment and during treatment, respectively. On average, pre-treatment dose measurements are performed once while transit dose measurements are done 1-2 times in the first week of the treatment series only. In the future, the transit dose measurements will be done weekly (Sec. 8.5).

The percentage of clipped pre-treatment fields is $13.4 \%$ which means that a large number of treatment fields can not be imaged for transit dose verification. Furthermore, the percentage of beam interrupts by the treatment machine is only $0.7 \%$, causing a portal image acquisition to be repeated in case of pre-treatment dose verification. In case of transit dose verification, the partially acquired portal image is not used for 2D portal dosimetry.

The clinical reliability of the technical infrastructure for 2D portal dosimetry is estimated to be $99 \%$ (based on measurements performed in October 2007). 


\section{REFERENCES}

1 D.A. Low, W.B. Harms, S. Mutic, and J.A. Purdy, "A technique for the quantitative evaluation of dose distributions," Med. Phys. 25, 656-661 (1998).

2 D.A. Low, and J.F. Dempsey, "Evaluation of the gamma dose distribution comparison method," Med. Phys. 30, 2455-2464 (2003).

3 R. Amor, "PACS overview: past, present, and future," Biomed. Instrum. Technol. 40, 281-282 (2006).

4 A. Badano, "AAPM/RSNA tutorial on equipment selection: PACS equipment overview: display systems," Radiographics 24, 879-889 (2004).

5 E. Samei, J.A. Seibert, K. Andriole, A. Badano, J. Crawford, B. Reiner, M.J. Flynn, and P. Chang, "AAPM/RSNA tutorial on equipment selection: PACS equipment overview: general guidelines for purchasing and acceptance testing of PACS equipment," Radiographics 24, 313-334 (2004).

6 J. Kabachinski, "DICOM: key concepts--part I," Biomed. Instrum. Technol. 39, 214-216 (2005).

7 J. Kabachinski, "DICOM: key concepts--part II," Biomed. Instrum. Technol. 39, 292-294 (2005). 
LIST OF ABBREVIATIONS 



\section{LIST OF ABBREVIATIONS}

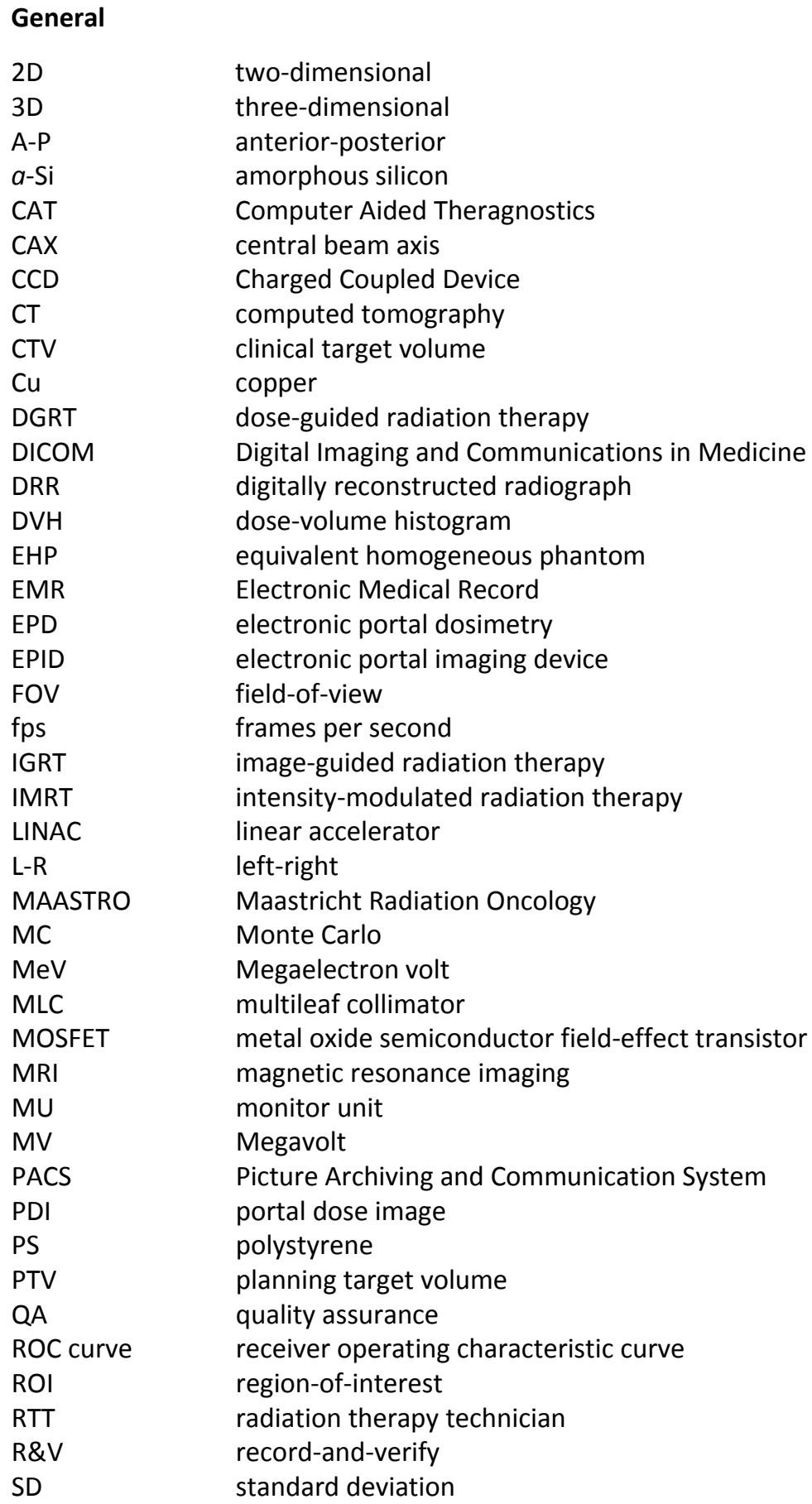

two-dimensional 
SDD

S-I

SNR

SPR

SSD

TLD

TME

TPS source-to-detector distance

superior-inferior

signal-to-noise ratio

scatter-to-primary ratio

source-to-skin distance

thermoluminescent dosimeter

total mesorectal excision

treatment planning system

\section{Point dose verification methods and application}

$\mu \quad$ linear attenuation coefficient

A off-axis wedge factor

c equivalent square field size

$c_{R} \quad$ reference field size

$D_{5} \quad$ in vivo dose at $5 \mathrm{~cm}$ depth in water

$D_{c} \quad$ absolute dose in water at the central beam axis

$\dot{D}_{R} \quad$ absorbed dose per monitor unit

$D_{T} \quad$ transit dose in water

$f_{R} \quad$ focus-surface distance

$k_{w} \quad$ wedge factor

$O_{R} \quad$ output ratio

$S P R_{1} \quad$ scatter-to-primary ratio behind a patient

$S P R_{2} \quad$ scatter-to-primary ratio at radiological depth of $5 \mathrm{~cm}$

$\mathrm{SSD}_{5} \quad$ focus-skin distance at the central beam axis

$t$

$u$

radiological thickness of a patient or phantom

number of monitor units

$X \quad$ field size defined by the $X$ pair of collimating jaws of a LINAC

$Y \quad$ field size defined by the $Y$ pair of collimating jaws of a LINAC

$z_{5} \quad$ geometrical depth at radiological depth of $5 \mathrm{~cm}$

$z_{R} \quad$ geometrical depth in water

\section{Two-dimensional transit dosimetry methods and application}

$\begin{array}{ll}\% D_{\min } & \text { relative change in DVH parameter, e.g. for } D_{\min } \\ \% D_{\min , \text { tol }} & \text { tolerance deciding if a change in e.g. } D_{\min } \text { is clinically relevant } \\ \alpha & \text { average photon energy } E \text { expressed in units } m_{0} c^{2}=0.511 \mathrm{MeV} \\ \gamma_{\max } & \text { maximum gamma value } \\ \gamma_{\operatorname{mean}} & \text { mean gamma value } \\ \gamma_{S D} & \text { standard deviation of the mean gamma value } \\ \gamma_{\%} & \text { percentage of gamma values larger than } 1 \\ \theta & \text { angle of scattered photons } \\ \mu & \text { linear attenuation coefficient } \\ A_{F} & \text { field area irradiating a patient volume } \\ A U C & \text { area under the curve } \\ B P & \text { beam profile correction }\end{array}$


$D_{\text {mean }}$

$D_{\text {min }}$

$D_{p}$

$f_{50}$

$F$

$F_{1}$

$F_{2}$

$G$

$G_{\text {raw }}$

$G_{\text {ref }}$

HI

I

$I^{M}$

$l^{P}$

$K$

$K_{F}$

$K_{\text {Gauss }}$

$K_{\text {Isotropic }}$

$K_{K N}$

$K_{S}$

NPV

O

$O_{S}$

$p$

$p_{\text {cutoff }}$

$P$

PPV

$r$

$r S P R$

$S$

$S$

SE

$S P$

$t$

$t^{A}$

$t^{E}$

$t_{\text {rad }}$

$T$

$T^{P}$

$T^{S}$

TERMA

$V_{x}$

square field size

conversion factor from absolute dose to EPID grayscale value

conformity index

midplane-to-detector distance

reference midplane-to-detector distance

maximum dose

mean dose

minimum dose

portal dose measured in water

critical frequency used to quantify image sharpness

energy spectrum correction

energy spectrum correction for primary portal dose differences

energy spectrum correction for patient scattered dose differences

grayscale value before image lag and ghosting correction

grayscale value after image lag and ghosting correction

reference grayscale value

heterogeneity index

total portal dose with an object in the beam

measured portal dose with an object in the beam

predicted portal dose with an object in the beam

scatter kernel

field size dependence kernel

scatter kernel based on Gaussian functions

scatter kernel based on an isotropic point source

scatter kernel based on single Compton scattered photons

scatter kernel

negative predictive value

portal dose without an object in the beam

scatter offset

probability that a DVH parameter change is clinically relevant

cutoff value to round the probability $p$ to 0 or 1

primary portal dose with an object in the beam

positive predictive value

off-axis position calculated by $v\left(x^{2}+y^{2}\right)$

residual scatter-to-primary ratio

scaling factor calculated by $\left(d / d^{\text {ref }}\right)^{2}$

phantom scattered portal dose with an object in the beam

sensitivity

specificity

radiological thickness of a patient or phantom

actual radiological thickness

extracted radiological thickness from predicted primary dose

irradiation time

transmission

component of transmission arising from primary dose

component of transmission arising from phantom scatter

total energy released per mass

relative volume receiving at least $\mathrm{x} \%$ of prescribed dose 

SUMMARY / SAMENVATTING 



\section{SUMMARY}

The goal of radiotherapy is to irradiate tumor tissue to a high dose value while limiting the dose to surrounding normal tissue as much as possible. In external megavolt photon beam treatment, radiotherapy is administered in a fractionated manner, which implies that every fraction should be given in a reproducible way and that the dose delivery in a patient should be as close as possible to the prescribed dose.

This work describes dose verification methods based on portal dose measurements using electronic portal imaging devices (EPIDs). Methods and application of both point dose verification and two-dimensional transit dosimetry in clinical routine are discussed.

\section{Point dose verification methods and application}

Point dose verification procedures have been investigated and applied clinically in our department to verify treatment parameter transfer, machine output and dose delivery to a patient. All procedures were based on EPID images that were acquired, analyzed and evaluated by radiation therapy technicians.

Dose values could be measured within 1-2\% compared to a reference dose measurement in water using a dosimetric calibration model for video-based EPIDs. These measured portal dose values were compared to predicted reference point dose values, which in general showed differences smaller than $3 \%$. However, this discrepancy could in some cases be larger due to limitations of the prediction models.

False positive dose delivery errors could be related to acquisition errors, user errors, implementation errors and procedure limitations. Using the recognized error sources, possible improvements of the verification procedures were discussed which should minimize all errors besides real dose delivery errors. Transfer and machine errors were found for $1 \%$ of all pre-treatment measurements. It was estimated that a true positive dose delivery error was found in $12 \%$ of all imaged treatment sessions during treatment. These patient related dose delivery errors were caused by set-up errors or organ motion, particularly in case of breast treatments but also for lung, and head and neck irradiations.

\section{Two-dimensional transit dosimetry methods and application}

A procedure for two-dimensional transit dosimetry has been developed which compares a predicted reference dose distribution in the EPID plane with a measured portal dose image, using the gamma evaluation method.

The extension of the point dose prediction model for transit dose values to a 2D portal dose prediction model has been discussed and its accuracy tested elaborately in an experimental study. The model is based on pencil beam scatter kernels, which are solely derived from phantom measurements. The model is able to predict 2D portal dose images behind a patient, based on a portal dose image without the patient in the beam in combination with the radiological thickness of the patient. If the radiological 
midplane of an irradiated object coincides with the isocenter plane, the accuracy of the model is better than $2 \%$ (mean difference). If this assumption is not valid, larger deviations will occur that can, however, be corrected. By using the 2D portal dose prediction model, a number of limitations encountered by the point dose prediction models could be solved.

A global dosimetric calibration procedure for indirect-detection $a$-Si EPIDs has been implemented to convert grayscale EPID images into full-scatter portal dose images. The model corrects for ghosting effects, field size dependence, beam profile loss, photon energy dependence and long-term drift of the EPID response. All phantom verification measurements satisfied gamma evaluation criteria of $3 \%$ and $3 \mathrm{~mm}$ across the entire EPID plane inside the radiation field. Out-of-field, larger dose differences were observed that could be minimized by deriving an energy dependent scatter kernel based on transmission values.

The ghosting effects for $a$-Si EPIDs were more elaborately studied by performing a comparison of ghosting effects for three commercial $a$-Si EPIDs. For all EPIDs, a relative under-response was observed at shorter irradiation times due to charge trapping, suggesting that the ghosting effects are a fundamental property of indirect-detection $a$-Si EPIDs.

The two-dimensional transit dosimetry procedure from this work has been applied to all patients treated with curative intent in our department. For conventional breast cancer treatment, a model has been developed that predicts dose-volume histogram (DVH) changes due to set-up errors using measured portal transit dose images for the planning target volume (PTV). Correlations between DVH changes and 2D gamma parameters have been investigated for different simulated set-up errors and described by a binomial logistic regression model. Clinical accuracy has been determined by applying the predictive model to clinically measured portal dose images. Changes in the mean PTV dose, $V_{90}$ and $V_{95}$ could be predicted based on a set of $2 \mathrm{D}$ gamma parameters. Most pronounced changes in the three DVH parameters were found for set-up errors in the lateral-medial direction. Clinical accuracy was decreased due to the occurrence of patient rotations or breast deformations during treatment, but the overall reliability of the predictive model remains high in those cases.

The work described in this thesis shows that routine portal dosimetry is feasible with a high accuracy. Furthermore, a wider variety of dose delivery errors can be found compared to the use of traditional point dosimeters. This is because portal dose images are two-dimensional, which solves the positioning problems of point dosimeters, and in addition also allows for the detection of dose differences in the entire EPID plane. 


\section{SAMENVATTING}

Het doel van radiotherapie is het bestralen van tumorweefsel tot een hoge stralingsdosis waarbij de dosis in het omringende normale weefsel zo veel mogelijk beperkt dient te worden. In het geval van bestraling met uitwendige bundels megavolt röntgenstraling wordt de radiotherapie in fracties uitgevoerd, waarin elke bestralingsfractie op reproduceerbare wijze gegeven moet worden om de verschillen tussen voorgeschreven en afgegeven dosis in een patiënt zo klein mogelijk te maken.

Dit proefschrift beschrijft dosisverificatiemethodes die gebaseerd zijn op dosismetingen met "Electronic Portal Imaging Devices" (EPID's). Methodieken en de toepassing in de klinische praktijk van zowel puntdosisverificatie als tweedimensionale transmissiedosimetrie zijn besproken.

\section{Methodes en toepassing van puntdosisverificatie}

Procedures voor puntdosisverificatie zijn onderzocht en klinisch toegepast om de overdracht van bestralingsparameters, de output van een behandelmachine en de dosisafgifte aan een patiënt te controleren. Alle procedures waren gebaseerd op EPID-afbeeldingen die door radiotherapeutische laboranten werden gemaakt, geanalyseerd en geëvalueerd.

Dosiswaarden konden gemeten worden door gebruik te maken van een kalibratiemodel voor "video-based" EPID's waarbij de dosisverschillen tussen EPID- en referentiedosismeting in water binnen 1-2\% lagen. Een gemeten EPID-dosiswaarde werd vergeleken met een voorspelde dosiswaarde waarbij de verschillen over het algemeen kleiner waren dan 3\%. Echter, door beperkingen van de voorspellingsmodellen konden deze verschillen in bepaalde gevallen ook groter zijn.

Een fout-positieve dosisafgifte kon veroorzaakt zijn door acquisitiefouten, gebruikersfouten, implementatiefouten en beperkingen in de procedures. Op basis van deze oorzaken zijn mogelijke verbeteringen van de verificatieprocedures besproken. Hierdoor zou de frequentie van een gedetecteerde fout-positieve dosisafgifte geminimaliseerd kunnen worden. Fouten in overdracht van bestralingsparameters en in de output van een behandelmachine werden gevonden voor $1 \%$ van alle pre-treatment metingen. Voor $12 \%$ van alle afgebeelde bestralingsfracties werden fouten opgespoord in de dosisafgifte aan een patiënt. Deze patiëntgerelateerde fouten werden veroorzaakt door onzekerheden in de positionering van een patiënt of interne orgaanbeweging. Met name bij borstbestralingen, maar ook bij bestralingen van het long- en hoofd/halsgebied, werden deze fouten gevonden.

\section{Methodes en toepassing van tweedimensionale transmissiedosimetrie}

Een procedure voor het uitvoeren van tweedimensionale transmissiedosimetrie is ontwikkeld waarbij een voorspelde dosisverdeling in het vlak van de EPID is vergeleken met een gemeten dosisbeeld. Voor de vergelijking werd de gamma evaluatiemethode gebruikt. 
De uitbreiding van het voorspellingsmodel voor transmissiedosiswaarden in een punt naar een 2D dosisvoorspellingsmodel is besproken en de nauwkeurigheid ervan uitvoerig getest in een experimentele studie. Het model is gebaseerd op "pencil-beam scatter kernels" die zijn bepaald met fantoommetingen. Het model was in staat om 2D dosisbeelden achter een patiënt te voorspellen door gebruik te maken van een dosisbeeld zonder de patiënt in de bundel en de radiologische dikte van de patiënt. De nauwkeurigheid van het model was beter dan $2 \%$ (gemiddelde verschil) als het vlak met het radiologische midden van de patiënt samenviel met het isocentrumvlak. Indien dit niet het geval was, konden grotere verschillen ontstaan die echter gecorrigeerd konden worden. Door het toepassen van het 2D dosisvoorspellingsmodel zijn een aantal beperkingen van de voorspellingsmodellen voor puntdosiswaarden opgelost.

Een generiek dosimetrisch kalibratiemodel voor indirecte detectie $a$-Si EPID's is geïmplementeerd om grijswaarden in EPID-afbeeldingen te converteren naar "full-scatter" dosisbeelden ter plekke van de EPID. Het model houdt rekening met "ghosting" effecten, afhankelijkheden van veldgrootte en energie, bundelprofielverlies en lange termijn verloop van het EPID-signaal. Alle verificatiemetingen met fantomen voldeden aan gamma evaluatiecriteria van $3 \%$ en $3 \mathrm{~mm}$ over het gehele EPID-oppervlak binnen de veldgrenzen. Buiten de veldgrenzen werden grotere dosisverschillen gevonden die verminderd kunnen worden door een energie-afhankelijke "scatter kernel" af te leiden op basis van transmissiewaarden.

De "ghosting" effecten voor $a$-Si EPID's zijn uitvoeriger bestudeerd door deze te vergelijken voor drie commerciële $a$-Si EPID's. Een relatieve signaalvermindering werd waargenomen voor alle EPID's in het geval van kortere bestralingstijden. Deze vermindering kon verklaard worden door "charge trapping", hetgeen suggereert dat de "ghosting" effecten een fundamentele eigenschap zijn van indirecte detectie $a$-Si EPID's.

De in dit werk beschreven procedure voor tweedimensionale transmissiedosimetrie is in ons instituut toegepast bij alle patiënten die behandeld werden met curatieve intentie. Een model werd ontwikkeld dat veranderingen in een dosis-volume histogram (DVH) voorspelt voor het "Planning Target Volume" (PTV) ten gevolge van set-up fouten voor de conventionele behandeling van borstkanker. Dit model was gebaseerd op gemeten transmissiedosisverdelingen met een EPID. Correlaties tussen DVH-veranderingen en 2D gammaparameters zijn onderzocht voor verschillende gesimuleerde "set-up" fouten, en zijn gemodelleerd met een binomiaal logistisch regressiemodel. De klinische nauwkeurigheid werd bepaald door het predictieve model toe te passen op klinisch gemeten EPID-dosisbeelden. Veranderingen in de gemiddelde PTV-dosis, $V_{90}$ en $V_{95}$ konden voorspeld worden op basis van een reeks 2D gammaparameters. De grootste veranderingen in de drie DVH-parameters werden gevonden voor "set-up" fouten in latero-mediale richting. De klinische nauwkeurigheid was lager in het geval van patiëntrotaties of vervormingen van de borst tijdens de behandeling, maar de globale betrouwbaarheid van het predictieve model bleef desalniettemin hoog. 
Het werk beschreven in dit proefschrift laat zien dat routine dosisverificatieprocedures gebaseerd op EPID-metingen mogelijk zijn met een hoge nauwkeurigheid. Bovendien kan een grotere verscheidenheid aan fouten in dosisafgifte gevonden worden in vergelijking met het gebruik van traditionele puntdosimeters. Door de vaste positie van de EPID worden de positioneringsproblemen van puntdosimeters vermeden. Tenslotte zijn de EPID-dosisverdelingen tweedimensionaal waardoor detectie van dosisverschillen over het gehele EPID-oppervlak mogelijk is. 

DANKWOORD 



\section{DANKWOORD}

Het uitvoeren van een promotie kan vergeleken worden met het componeren van een muziekstuk ofwel het schrijven van een partituur voor een groot muziekorkest. De compositie moet bestaan uit verschillende delen met onderlinge samenhang en bovendien een vernieuwend karakter hebben. Het uiteindelijke resultaat van mijn promotie is een muziekwerk geworden dat aan deze voorwaarden voldoet en bovendien voor een breed publiek (patiënten) al is uitgevoerd. Omdat hierbij veel mensen waren betrokken, is een woord van dank op zijn plaats.

Een compositie is gebonden aan regels en algemeen geldende normen die in de gaten zijn gehouden door mijn promotor Philippe Lambin en mijn copromotores André Minken, Ben Mijnheer en André Dekker.

Beste Philippe, jij bent naast opdrachtgever ook de grote inspirator geweest tot het schrijven van deze partituur na het afronden van mijn opleiding tot klinisch fysicus in 2002. Je onderkende de behoefte naar een vernieuwende muziek en was altijd duidelijk dat deze compositie uiteindelijk ook uitgevoerd moest worden. Op de juiste momenten kon je mijn werkkamer binnen lopen en daarmee de vaart in het compositieproces houden.

Beste André (M.), vanaf het begin ben je betrokken geweest bij deze compositie. Lange discussies konden we voeren op je kamer achter een groot white board over het aanpakken, uitwerken en uitvoeren van een muziekdeel. Daarnaast hebben we vaak nog tot 's avonds laat doorgewerkt om nieuwe inzichten experimenteel te toetsen. Jouw enthousiasme heeft me door moeilijke periodes heen geholpen waarin er weinig tijd was voor componeren door de verhuizing naar een nieuw concertgebouw en het moeten stemmen van een heleboel nieuwe muziekinstrumenten.

Beste Ben, in een compositie kunnen tempowisselingen een werk een bepaald karakter geven en spannend houden. Het tempo waarmee jij de verschillende delen van de partituur las en van commentaar voorzag, was altijd hoog. In een rustig en bedacht tempo gaf je jouw kritische noten en met je grote kennis op het gebied van andere muziekstromingen heb je dit werk karakter gegeven.

Beste André (D.), jij bent pas later betrokken geraakt bij het schrijven van deze partituur maar je invloed is duidelijk te merken. Het laatste deel van deze compositie heeft een brug geslagen van deze muziekstroming naar een belangrijke stroming die op dit moment onderzocht wordt in ons concertgebouw. Jouw heldere analyse heeft de muziek bovendien transparanter gemaakt.

Beste Wouter, als collega-componist en nu als paranimf hebben we vaak overlegd over inhoud en aanpak. Inspiratie hebben we o.a. opgedaan in Melbourne en San Francisco waar internationale muziekstromingen en het edele backgammon zijn bestudeerd. De afgelopen jaren werd duidelijk dat onze stroming ook internationaal gewaardeerd is geworden. Op dit moment heb je gekozen voor een andere richting maar ik weet zeker dat nieuwe composities van jouw hand zeer herkenbaar kunnen blijven door hun hoge 
kwaliteit. Daarnaast zul je als gastdirigent in de toekomst nog bij het uitvoeren van onze composities optreden.

Verder wil ik graag alle studenten en co-auteurs bedanken die betrokken zijn geweest bij het ontwikkelen en schrijven van de verschillende muziekdelen, met name Chris, Karin, Marjon, laïn, Leah en Lucas.

Een compositie verdient het niet om te eindigen in een stoffig muziekarchief maar dient uitgevoerd te worden door een goed muziekorkest. De première van dit muziekwerk is uiteindelijk uitgevoerd in het concertgebouw MAASTRO CLINIC waarbij het orkest gevormd is door laboranten, radiotherapeut-oncologen en klinisch fysici. Zoals bij elk vernieuwend muziekwerk kan de interpretatie ervan door iedereen verschillen en kost het tijd voordat de compositie door het orkest omarmd wordt. Het enthousiasme waarmee de compositie op dit moment wordt uitgevoerd voor een publiek van 1200 patiënten per jaar, geeft mij echter veel voldoening. Hiervoor wil ik alle orkestleden bedanken, met name Claudia en Esther voor hun hulp bij de première.

Een speciaal woord van dank gaat uit naar mijn directe collega's Brigitte, Erik, Geert, Lars, Martijn, Michel, Miguel, Peter, Ramon en Robert. Jullie hebben een aantal van mijn klinische werkzaamheden overgenomen tijdens het componeren van deze partituur. Bovendien vind ik het mooi om te zien hoe jullie dit muziekstuk promoten.

De uitvoering van dit muziekwerk, en alle andere uitvoeringen in ons concertgebouw, zouden niet mogelijk zijn zonder de werkzaamheden van een heleboel mensen op de achtergrond. Een belangrijke bijdrage hierin wordt geleverd door de mensen van de TiQc. Jongens, jullie allemaal bedankt voor het stemmen van de muziekinstrumenten, het monitoren van de klankverhoudingen en een stuk gezelligheid. Rianne en Simone, jullie bedankt voor de secretariële ondersteuning.

De compositie en uitvoering is tot in detail beoordeeld door recensenten, te weten prof. Wildberger, prof. ter Haar Romeny, prof. van Herk, prof. Pouliot en dr. Verhaegen. Vanaf deze plaats wil ik hen bedanken voor hun kritieken.

Beste Jo, leuk dat je als paranimf mij in de finale van deze compositie hebt bijgestaan met je brede muziekkennis bij het maken van enkele stellingen.

Bij het schrijven van de compositie, maar zeker bij het uitvoeren ervan, was af en toe overredingskracht, veel discipline en volhouden nodig. Dit zonder voorbij te gaan aan de belangrijke dingen in het leven. Hierbij heb ik veel gehad aan de lessen die mijn familie mij heeft bijgebracht en waar ik hen voor wil bedanken als de belangrijkste achterban die ik heb.

Lieve Iverna. Het componeren naast de dagelijkse werkzaamheden als klinisch fysicus betekende vrije tijd opofferen. Altijd heb je me hierin gesteund, zelfs met de komst van Julia en Tobias, en kon je mijn beslommeringen met een vrolijke noot relativeren. Echte progressie werd gemaakt toen ik twee dagen per week thuis kon werken. Op die dagen merkte ik dat de leercurve van een pasgeborene steiler is dan die van een promovendus en ook dat niet-werkende moeders niet bestaan. 
CURRICULUM VITAE 

Sebastiaan (Bas) Martin Jan Jacques Geert Nijsten is geboren op 6 april 1975 in Geleen en groeide op in Beek (Lb). In 1993 behaalde hij zijn diploma voor het Atheneum aan Scholengemeenschap Groenewald in Stein en aansluitend startte hij met de studie Technische Natuurkunde aan de Technische Universiteit Eindhoven (TU/e). In februari 1998 rondde hij deze studie succesvol af met een onderzoek naar mogelijke beeldartefacten bij gebruik van MRA op de afdeling Klinische Fysica van het St. Joseph ziekenhuis in Veldhoven (het huidige Maxima Medisch Centrum). Aansluitend verrichtte hij als medewerker van de vakgroep Data Acquisitie and Klinische Fysica binnen de Faculteit Technische Natuurkunde van de TU/e een simulatiestudie naar mogelijke toepassingen van de configuratietheorie bij conventionele MRI pulssequenties. In september 1998 startte hij met de opleiding tot Klinisch Fysicus op het werkterrein Radiotherapie in het Radiotherapeutisch Instituut Limburg (de huidige MAASTRO CLINIC) met als opleiders Drs. R.J. de Vos en Dr. I.A.D. Bruinvis. Integraal onderdeel van de opleiding was een tweejarige postdoctorale opleiding Fysische Instrumentatie aan het Stan Ackermans Instituut van de TU/e die in september 2001 werd afgerond met het behalen van de graad Master of Technological Design. Tijdens deze opleiding verrichtte hij gedurende een periode van 3 maanden onderzoek naar het modelleren van lichttransport in een menselijke vinger door pulsoximetrie binnen de Future Developments Group van de Oximetry Business Unit binnen Datex-Ohmeda, Louisville, Colorado in de Verenigde Staten. Ook werd een 9 maanden durend onderzoek verricht binnen het Radiotherapeutisch Instituut Limburg naar het gebruik van electronic portal imaging als instrument voor kwaliteitscontroles binnen de radiotherapie, hetgeen de aanleiding vormde voor het onderzoek zoals beschreven in dit proefschrift. De registratie als Klinisch Fysicus behaalde hij in 2002 waarna hij in dienst trad als Klinisch Fysicus binnen MAASTRO CLINIC met de aandachtsgebieden portal imaging, portal dosimetry, (MV) cone beam CT en digitalisering. 

LIST OF PUBLICATIONS 



\section{PUBLISHED ARTICLES}

1 S.M.J.J.G. Nijsten, A.W.H. Minken, P. Lambin, and I.A.D. Bruinvis, "Verification of treatment parameter transfer by means of electronic portal dosimetry," Med. Phys. 31, 341-347 (2004).

2 A. van der Wel, S.M.J.J.G. Nijsten, M. Hochstenbach, R. Lamers, L.J. Boersma, R. Wanders, L. Lutgens, M. Zimny, S.M. Bentzen, B. Wouters, P. Lambin, and D. De Ruysscher, "Increased therapeutic ratio by 18FDG-PET CT planning in patients with clinical CT stage N2-N3M0 non-small cell lung cancer: a modeling study," Int. J. Radiat. Oncol. Biol. Phys. 61, 649-655 (2005).

3 W.J.C. van Elmpt, S.M.J.J.G. Nijsten, B.J. Mijnheer, and A.W.H. Minken, "Experimental verification of a portal dose prediction model," Med. Phys. 32, 2805-2818 (2005).

4 D. De Ruysscher, S. Wanders, A. Minken, A. Lumens, J. Schiffelers, C. Stultiens, S. Halders, L.J. Boersma, A. Baardwijk, T. Verschueren, M. Hochstenbach, G. Snoep, B. Wouters, S.M.J.J.G. Nijsten, S.M. Bentzen, M. Kroonenburgh, M. Ollers, and P. Lambin, "Effects of radiotherapy planning with a dedicated combined PET-CT simulator of patients with non-small cell lung cancer on dose limiting normal tissues and radiation dose-escalation: A planning study," Radiother. Oncol. 77, 5-10 (2005).

5 C. Duisters, H. Beurskens, S.M.J.J.G. Nijsten, M.W.H. Starmans, S. Wanders, T. Verschueren, P. Lambin, A. Minken, and D. De Ruysscher, "Palliative chest irradiation in sitting position in patients with bulky advanced lung cancer," Radiother. Oncol. 79, 285-287 (2006).

6 L.N. McDermott, S.M.J.J.G. Nijsten, J.J. Sonke, M. Partridge, M.B. van Herk, and B.J. Mijnheer, "Comparison of ghosting effects for three commercial $a$-Si EPIDs," Med. Phys. 33, 2448-2451 (2006).

7 W.J.C. van Elmpt, S.M.J.J.G. Nijsten, R.F.H. Schiffeleers, A.L.A.J. Dekker, B.J. Mijnheer, P. Lambin, and A.W.H. Minken, "A Monte Carlo based three-dimensional dose reconstruction method derived from portal dose images," Med. Phys. 33, 2426-2434 (2006).

8 S.M.J.J.G. Nijsten, B.J. Mijnheer, A.L.A.J. Dekker, P. Lambin, and A.W.H. Minken, "Routine individualised patient dosimetry using electronic portal imaging devices," Radiother. Oncol. 83, 65-75 (2007).

9 W.J.C. van Elmpt, S.M.J.J.G. Nijsten, A.L.A.J. Dekker, B.J. Mijnheer, and P. Lambin, "Treatment verification in the presence of inhomogeneities using EPID-based three-dimensional dose reconstruction," Med. Phys. 34, 2816-2826 (2007). 
10 S.M.J.J.G. Nijsten, W.J.C. van Elmpt, M. Jacobs, B.J. Mijnheer, A.L.A.J. Dekker, P. Lambin, and A.H.W. Minken, "A global calibration model for $a$-Si EPIDs used for transit dosimetry," Med. Phys. 34, 3872-3884 (2007).

11 W.J.C. van Elmpt, S.M.J.J.G. Nijsten, B.J. Mijnheer, A.L.A.J. Dekker, and P. Lambin, "The next step in patient-specific QA: 3D dose verification of conformal and intensity-modulated RT based on EPID dosimetry and Monte Carlo dose calculations," Radiother. Oncol. 86, 86-92 (2008).

12 S.F. Petit, W.J.C. van Elmpt, S.M.J.J.G. Nijsten, P. Lambin, and A.L.A.J. Dekker, "Calibration of megavoltage cone-beam CT for radiotherapy dose calculations: Correction of cupping artifacts and conversion of CT numbers to electron density," Med. Phys. 35, 849- 865 (2008).

13 W.J.C. van Elmpt, L.N. McDermott, S.M.J.J.G. Nijsten, M. Wendling, P. Lambin, and B.J. Mijnheer, "A literature review of electronic portal imaging for radiotherapy dosimetry", Radiother. Oncol. 88, 289-309 (2008).

14 S.M.J.J.G. Nijsten, W.J.C. van Elmpt, B.J. Mijnheer, A.W.H. Minken, L.C.G.G. Persoon, P. Lambin, and A.L.A.J. Dekker, "Prediction of DVH parameter changes due to set-up errors for breast cancer treatment based on 2D portal dosimetry," Med. Phys. 36, 83-94 (2009).

15 W.J.C. van Elmpt, S.M.J.J.G. Nijsten, S.F. Petit, B.J. Mijnheer, P. Lambin, and A.L.A.J. Dekker, "3D in vivo dosimetry using megavoltage cone-beam CT and EPID dosimetry," Int. J. Radiat. Oncol. Biol. Phys. 73, 1580-1587 (2009).

\section{PUBLISHED ABSTRACTS}

1 A. van der Wel, S.M.J.J.G. Nijsten, M. Hochstenbach, L. Lutgens, R. Lamers, P. Lambin, M. Zimny, and D. De Ruysscher, "Reduction of lung toxicity and geographical miss by optimising 3-D radiotherapy treatment planning for non-small cell lung cancer (NSCLC) using FDG-PET scan," ESTRO-21, Prague, Radiother. Oncol. 64 Suppl. 1, s259 (2002).

2 A.W.H. Minken, S.M.J.J.G. Nijsten, E.J. Bloemen-van Gurp, W.F.J. du Bois, and P. Lambin, "Comparison of MOSFET and EPID-dosimetry for the evaluation of prostate treatment," ESTRO-21, Prague, Radiother. Oncol. 64 Suppl. 1, s43 (2002).

3 J. Steenhuijsen, J. Hermans, D. Jalink, M. Kunze, A.W.H. Minken, S.M.J.J.G. Nijsten, and I.A.D. Bruinvis, "Verification of dose calculations for asymmetrical (wedged) photon beams with the Focus convolution model," ESTRO-21, Prague, Radiother. Oncol. 64 Suppl. 1, s95 (2002). 
4 A. van der Wel, S.M.J.J.G. Nijsten, S.M. Bentzen, M. Hochstenbach, U. Büll, L. Lutgens, R. Wanders, B. Wouters, P. Lambin, and D. De Ruysscher, "Increased Tumor Control Probability (TCP) and radiation dose escalation by FDG-PET planning of patients with N2/N3 MO non-small cell lung cancer (NSCLC): A modeling study," ECCO-12, Copenhagen (2003).

5 S.M.J.J.G. Nijsten, A.W.H. Minken, P. Lambin, and I.A.D. Bruinvis, "Verification of treatment parameter transfer by means of Electronic Portal Dosimetry," AAPM $45^{\text {th }}$ Annual Meeting, San Diego, USA (2003).

6 S.M.J.J.G. Nijsten, B.J. Mijnheer, P. Lambin, and A.W.H. Minken, "Routine in-vivo dosimetry using electronic portal imaging devices (EPIDs): the MAASTRO experience," ESTRO-23, Amsterdam, Radiother. Oncol. 73 Suppl. 1, (2004).

7 W.J.C. van Elmpt, S.M.J.J.G. Nijsten, B.J. Mijnheer, and A.W.H. Minken, "Accuracy of primary portal dose extraction for in-vivo dosimetry purposes," ESTRO-23, Amsterdam, Radiother. Oncol. 73 Suppl. 1, s114-115 (2004).

8 D. Eekers, M. Kunze, M. Velders, J. Jager, S.M.J.J.G. Nijsten, A.W.H. Minken, L.J. Boersma, and P. Lambin, "Comparison of 2 dose calculation algorithms in tangential field irradiation for breast cancer," ESTRO-23, Amsterdam, Radiother. Oncol. 73 Suppl. 1, (2004).

9 C. Duisters, H. Beurskens, S.M.J.J.G. Nijsten, M.W.H. Starmans, S. Wanders, L.J. Boersma, T. Verschueren, P. Lambin, A.W.H. Minken, and D. De Ruysscher, "Decreasing lung exposure of patients suffering from advanced NSCLC by palliative irradiation in sitting position," $11^{\text {th }}$ World Conference on Lung Cancer (IASLC), Barcelona (2005).

10 M.W.H. Starmans, A.L.A.J. Dekker, S.M.J.J.G. Nijsten, A.W.H. Minken, and P. Lambin, "IMRT pre-treatment verification with ionization chamber, film and EPID: Quality vs. time consumption," AAPM $47^{\text {th }}$ Annual Meeting, Seattle, USA (2005).

11 M. Jacobs, S.M.J.J.G. Nijsten, P. Lambin, and A.W.H. Minken, "Dosimetric calibration of a Siemens OptiVue 500 amorphous silicon electronic portal imaging device," ESTRO $8^{\text {th }}$ Biennial Meeting, Lisboa (2005).

12 K.W. Lamberts, S.M.J.J.G. Nijsten, P. Lambin, and A.W.H. Minken, "Qualitative determination of errors causing portal transit dose differences using gamma evaluation parameters," ESTRO $8^{\text {th }}$ Biennial Meeting, Lisboa (2005).

13 W.J.C. van Elmpt, S.M.J.J.G. Nijsten, R.F.H. Schiffeleers, A.L.A.J. Dekker, B.J. Mijnheer, P. Lambin, and A.W.H. Minken, "Three-dimensional dose reconstruction for IMRT pre-treatment verification using portal dose images," EPI2K6 $9^{\text {th }}$ International Workshop on Electronic Portal Imaging, Melbourne, Australia (2006). 
14 S.M.J.J.G. Nijsten, W.J.C. van Elmpt, B.J. Mijnheer, P. Lambin, and A.W.H. Minken, "A correction model for spectral response of an a-Si EPID during exit-dosimetry," EPI2K6 $9^{\text {th }}$ International Workshop on Electronic Portal Imaging, Melbourne, Australia (2006).

15 C. Dehing, H. van der Weide, S. Wanders, L.J. Boersma, D. De Ruysscher, S.M.J.J.G. Nijsten, H. Steck, S. Krishnan, B. Rao, and P. Lambin, "Combining clinical data with literature data to improve prediction models for NSCLC after (chemo)radiotherapy," ASTRO $48^{\text {th }}$ Annual Meeting, Philadelphia, USA (2006).

16 S.F. Petit, W.J.C. van Elmpt, S.M.J.J.G. Nijsten, A.L.A.J. Dekker, and P. Lambin, "Various procedures for the reduction of cupping artifacts in MegaVoltage Cone-Beam CT," ESTRO-25, Leipzig, Radiother. Oncol. 81 Suppl. 1, s561 (2006).

17 S.M.J.J.G. Nijsten, B.J. Mijnheer, A.L.A.J. Dekker, P. Lambin, and A.W.H. Minken, "Routine in vivo dosimetry using Electronic Portal Imaging Devices," ESTRO-25, Leipzig, Radiother. Oncol. 81 Suppl. 1, (2006).

18 W.J.C. van Elmpt, S.M.J.J.G. Nijsten, B.J. Mijnheer, A.L.A.J. Dekker, and P. Lambin, "Pre-treatment verification using 3D dose reconstruction based on EPID measurements," ESTRO-25, Leipzig, Radiother. Oncol. 81 Suppl. 1, s388 (2006).

19 C. Dehing, H. van der Weide, D. De Ruysscher, S.M.J.J.G. Nijsten, S. Wanders, L.J. Boersma, H. Steck, S. Krishnan, R.B. Rao, and P. Lambin, "Combining clinical data with medical knowledge improves prediction models for NSCLC after (chemo-) radiotherapy, the models being more accurate and more consistent then the doctors," ESTRO-25, Leipzig, Radiother. Oncol. 81 Suppl. 1, (2006).

20 H. Steck, C. Dehing, H. van der Weide, D. De Ruysscher, S.M.J.J.G. Nijsten, S. Wanders, L.J. Boersma, S. Krishnan, R.B. Rao, and P. Lambin, "Models combining clinical data with medical knowledge are more accurate than doctors in predicting NSCLC after (chemo-) radiotherapy," ESTRO-25, Leipzig, Radiother. Oncol. 81 Suppl. 1, (2006).

21 W.J.C. van Elmpt, S.M.J.J.G. Nijsten, B.J. Mijnheer, A.L.A.J. Dekker, and P. Lambin, "The next step in patient-specific QA: 3D dose verification for conformal and intensity modulated RT based on EPID dosimetry and Monte Carlo dose calculations," ESTRO Physics, Barcelona, Radiother. Oncol. 84 Suppl. 1, s96 (2007).

22 W.J.C. van Elmpt, S.M.J.J.G. Nijsten, B.J. Mijnheer, A.L.A.J. Dekker, and P. Lambin, "The Next Step in Patient Specific QA: 3D Dose Verification for Conformal and Intensity Modulated RT Based on EPID Dosimetry and Monte Carlo Calculations," ASTRO $49^{\text {th }}$ Annual Meeting, Los Angeles, USA, Int. J. Radiat. Oncol. Biol. Phys. 69 Suppl. 1, s672-673 (2007). 
23 S.M.J.J.G. Nijsten, W.J.C. van Elmpt, B.J. Mijnheer, A.L.A.J. Dekker, P. Lambin, and A.W.H. Minken, "An energy spectrum response correction model for $a$-Si EPIDs used for transit dosimetry," ESTRO Physics, Barcelona, Radiother. Oncol. 84 Suppl. 1, s162 (2007).

24 H. Steck, C. Dehing, H. van der Weide, D. De Ruysscher, S.M.J.J.G. Nijsten, S. Wanders, L.J. Boersma, G. Fung, B. Krishnapuram, S. Krishnan, R.B. Rao, and P. Lambin, "A knowledge-model for predicting radiation-induced esophagitis," ASTRO $49^{\text {th }}$ Annual Meeting, Los Angeles, USA, Int. J. Radiat. Oncol. Biol. Phys. 69 Suppl. 1, (2007).

25 H. Steck, C. Dehing, H. van der Weide, D. De Ruysscher, S.M.J.J.G. Nijsten, S. Wanders, L.J. Boersma, S. Krishnan, R.B. Rao, and P. Lambin, "Osteopontin Level to Creatinine Clearance Predicts Survival of NSCLC Patients," ASTRO $49^{\text {th }}$ Annual Meeting, Los Angeles, USA, Int. J. Radiat. Oncol. Biol. Phys. 69 Suppl. 1, (2007).

26 W.J.C. van Elmpt, S.F. Petit, S.M.J.J.G. Nijsten, B.J. Mijnheer, P. Lambin, and A.L.A.J. Dekker, "3D in-vivo dose reconstruction using EPID measurements and MegaVoltage cone-beam CT scans," EPI2K8 $10^{\text {th }}$ International Workshop on Electronic Portal Imaging, San Francisco, USA (2008).

27 S.M.J.J.G. Nijsten, W.J.C. van Elmpt, R. Kollaard, L.C.G.G. Persoon, B.J. Mijnheer, A.L.A.J. Dekker, and P. Lambin, "Routine 2-D in vivo verification using EPID dosimetry: One year clinical experience at MAASTRO," EPI2K8 $10^{\text {th }}$ International Workshop on Electronic Portal Imaging, San Francisco, USA (2008).

28 S.M.J.J.G. Nijsten, W.J.C. van Elmpt, R. Kollaard, L.C.G.G. Persoon, B.J. Mijnheer, A.L.A.J. Dekker, and P. Lambin, "Routine 2-D in vivo verification using EPID dosimetry: One year clinical experience at MAASTRO," IPEM Biennial Radiotherapy Meeting, Bath (2008).

29 S.M.J.J.G. Nijsten, W.J.C. van Elmpt, B.J. Mijnheer, L.C.G.G. Persoon, P. Lambin, and A.L.A.J. Dekker, "2D in-vivo verification in clinical routine using EPID dosimetry: clinical experience with more than 2000 patients," ESTRO Physics, Göteborg, Radiother. Oncol. 88 Suppl. 2, s159 (2008).

30 W.J.C. van Elmpt, S.M.J.J.G. Nijsten, S.F. Petit, B.J. Mijnheer, P. Lambin, and A.L.A.J. Dekker, "A method to perform 3D in vivo dosimetry using MV cone-beam CT and EPID dosimetry: from planned to measured dose distribution," ESTRO Physics, Göteborg, Radiother. Oncol. 88 Suppl. 2, s160-161 (2008). 
